\title{
Measuring and tracking suspended sediment on tidal flats and inundated marshland: Relevance for vertical accretion rates and coastal protection measures on the North Frisian Halligen
}

\author{
Dissertation
}

zur Erlangung des

mathematisch-naturwissenschaftlichen Doktorgrades

"Doctor rerum naturalium"

der Georg-August-Universität Göttingen

im Promotionsprogramm Geowissenschaften

der Georg-August University School of Science (GAUSS)

\author{
vorgelegt von \\ Ingo Jürgen Hache \\ aus Freiburg im Breisgau
}

Göttingen 2020 
Betreuungsausschuss:

Prof. Dr. Hilmar von Eynatten, Abt. für Sedimentologie und Umweltgeologie, Geowissenschaftliches Zentrum, Georg-August-Universität Göttingen

Dr. Volker Karius, Abt. für Sedimentologie und Umweltgeologie, Geowissenschaftliches Zentrum, Georg-August-Universität Göttingen

Mitglieder der Prüfungskommission:

Referent: Prof. Dr. Hilmar von Eynatten, Abt. für Sedimentologie und Umweltgeologie, Geowissenschaftliches Zentrum, Georg-August-Universität Göttingen

Korreferent: Prof. Dr. Volker Thiel, Abt. für Geobiologie, Geowissenschaftliches Zentrum, Georg-August-Universität Göttingen

weitere Mitglieder der Prüfungskommission:

Prof. Dr. Daniela Sauer, Abt. für Physische Geographie, Geowissenschaftliches Zentrum, GeorgAugust-Universität Göttingen

Prof. Dr. Jonas Kley, Abt. für Strukturgeologie und Geodynamik, Geowissenschaftliches Zentrum, Georg-August-Universität Göttingen

J.-Prof. Dr.-Ing. Arne Arns, Agrar- und Umweltwissenschaftliche Fakultät, Universität Rostock

Dr. Volker Karius, Abt. für Sedimentologie und Umweltgeologie, Geowissenschaftliches Zentrum, Georg-August-Universität Göttingen

Tag der mündlichen Prüfung: 30. November 2020 
Hiermit erkläre ich an Eides statt, die vorliegende Arbeit selbstständig angefertigt zu haben und dabei keine anderen als die von mir angegebenen Quellen und Hilfsmittel benutzt zu haben.

Ferner erkläre ich, dass ich nicht anderweitig versucht habe, eine Dissertation einzureichen. 


\section{Contents}

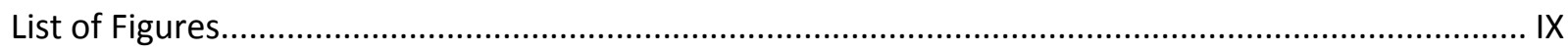

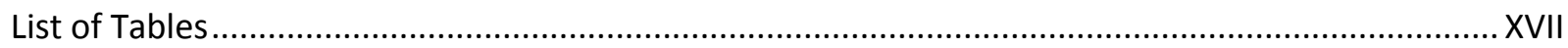

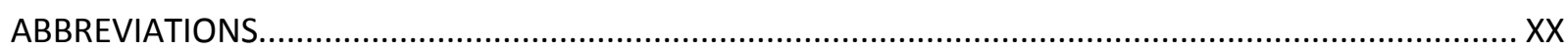

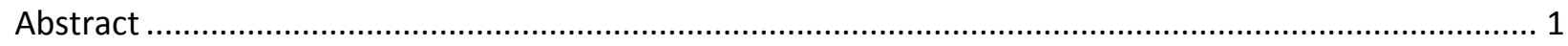

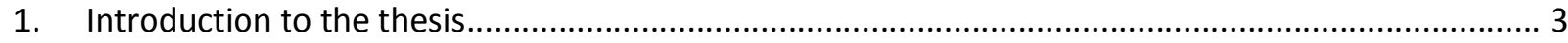

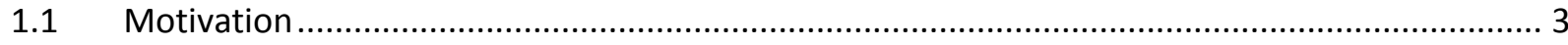

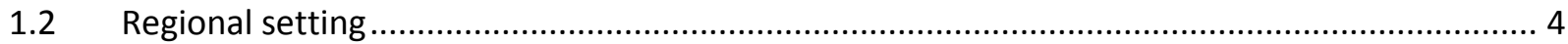

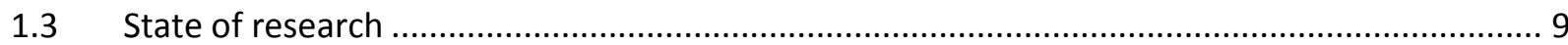

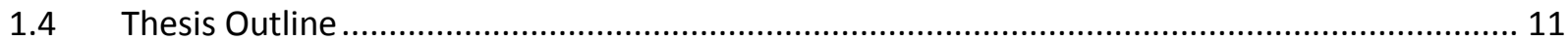

2 The development and application of an autonomous working turbidity measurement network: Assessing the spatial and temporal distribution of suspended particulate matter on tidal flats in the North Frisian Wadden Sea.

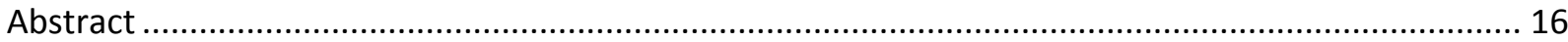

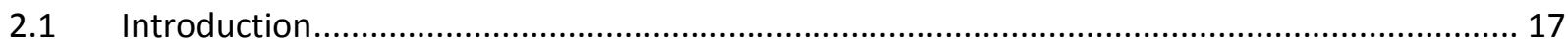

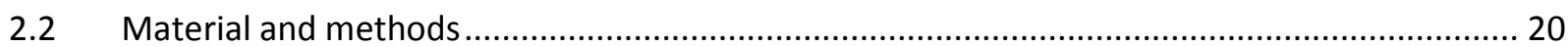

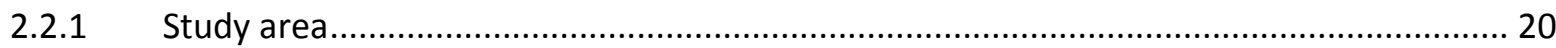

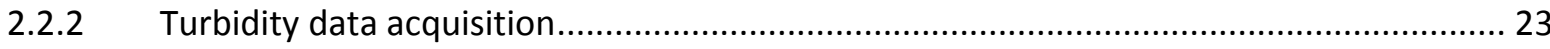

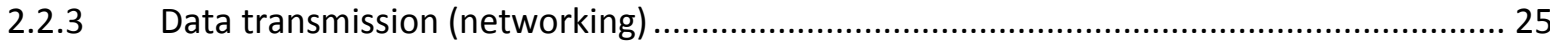

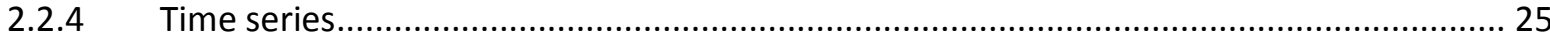

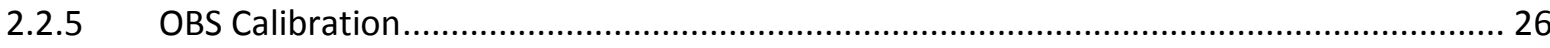

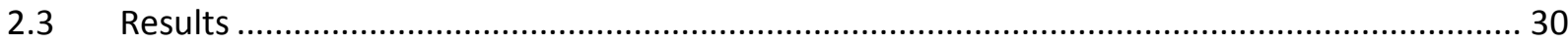

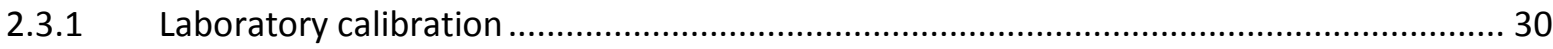

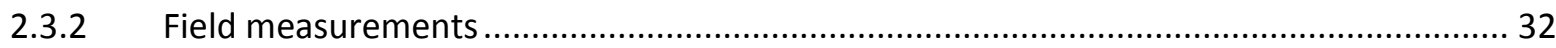

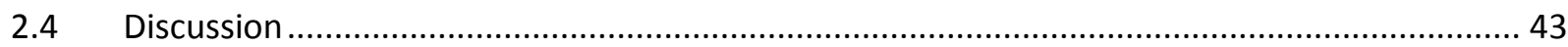

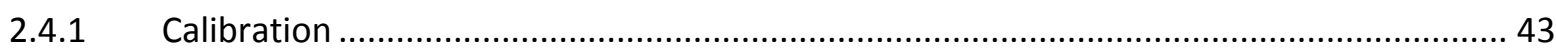

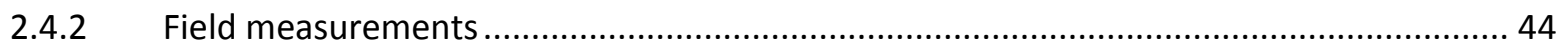

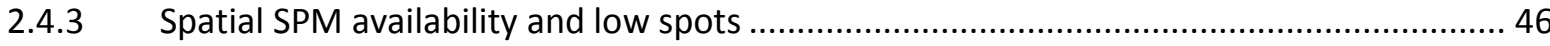

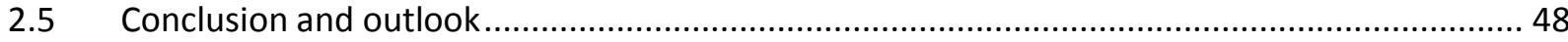

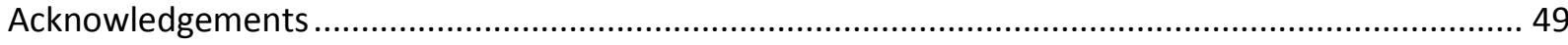

3 Suspended particulate matter for sediment accumulation on inundated anthropogenic marshland in the southern North Sea - Potential, thresholds and limitations ..................................................... 51

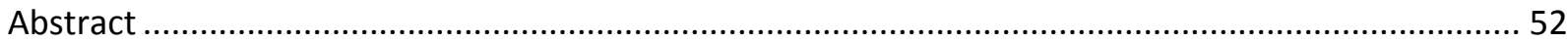

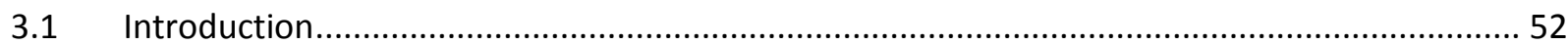

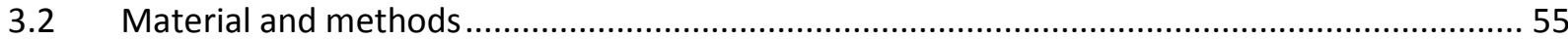




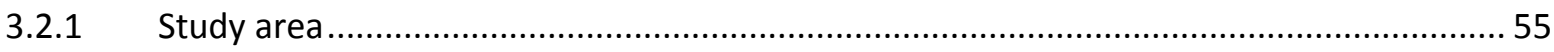

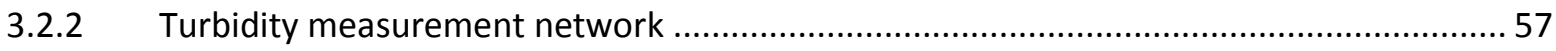

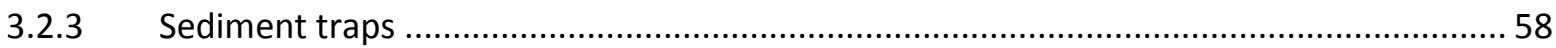

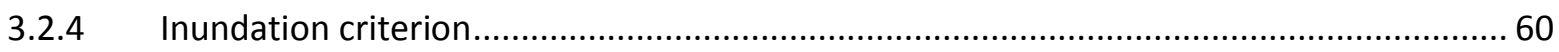

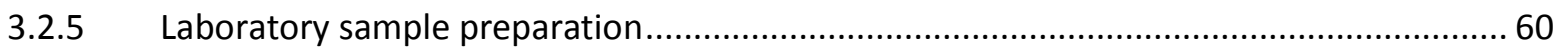

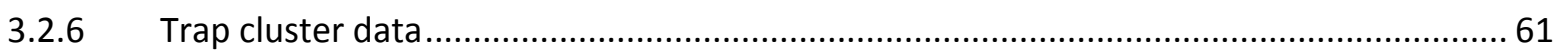

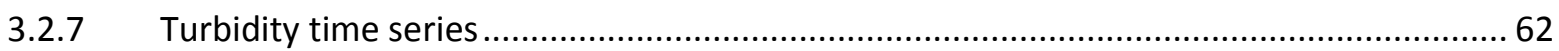

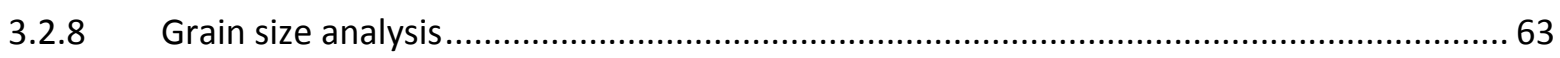

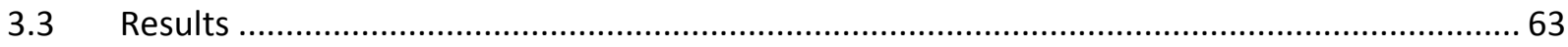

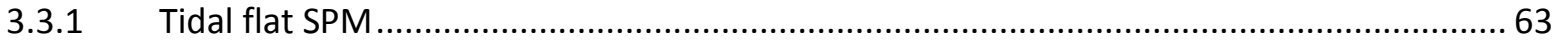

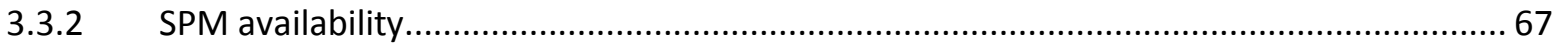

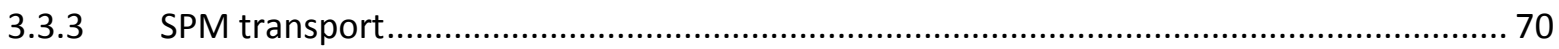

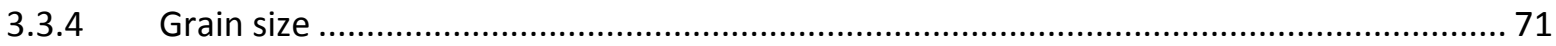

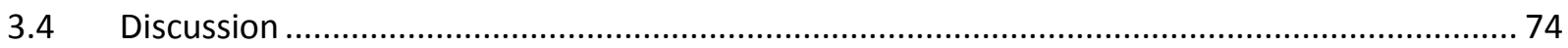

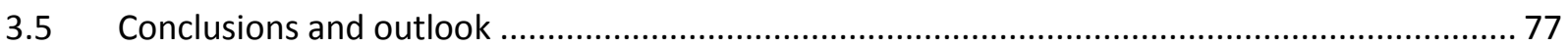

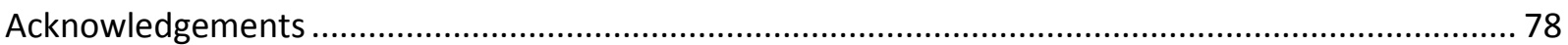

4 Assessing sediment accumulation at inundated anthropogenic marshland in the southeastern North Sea: using particle tracking on modified coastal protection structures ....................................81

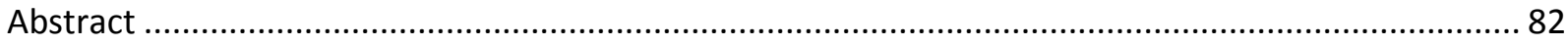

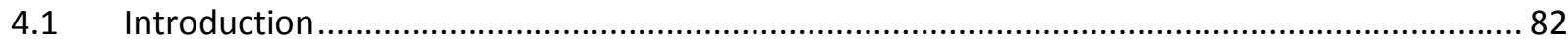

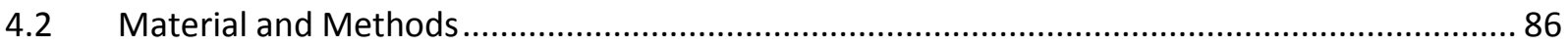

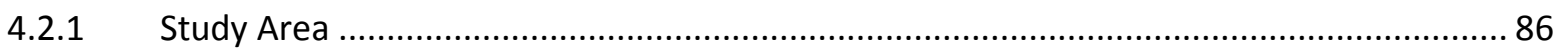

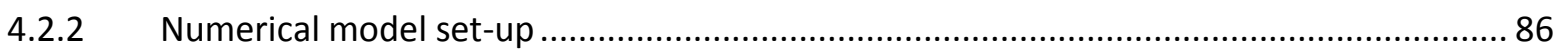

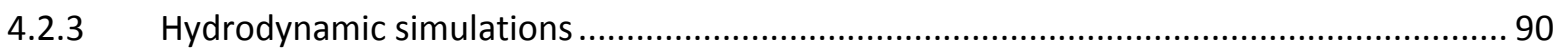

4.2.4 Particle classes and parametrization ............................................................... 91

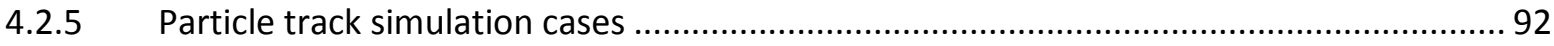

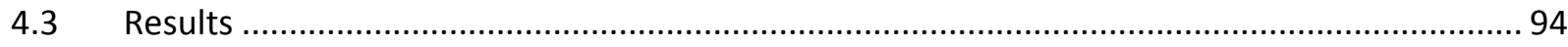

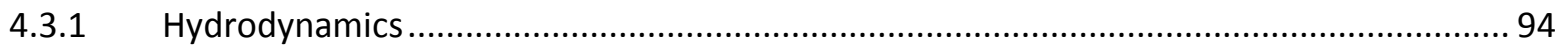

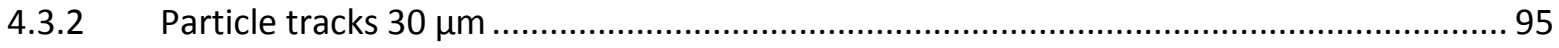

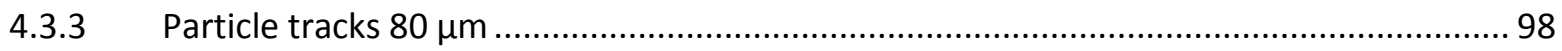

4.3.4 Particle accumulation versus peak high water level ................................................99

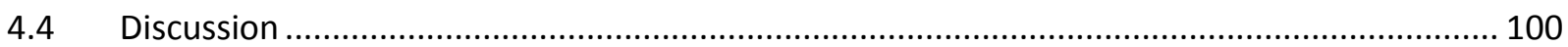

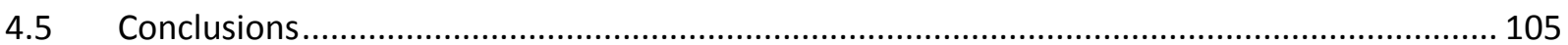

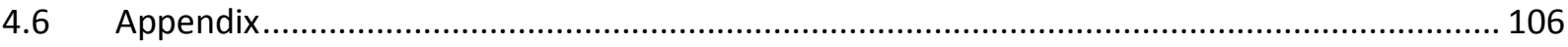

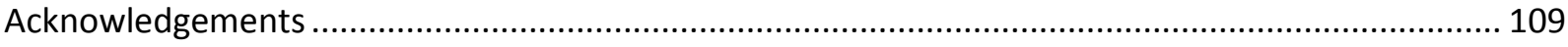


5 Storm surge induced sediment accumulation on marsh islands in the southeastern North Sea: Implications for coastal protection

Abstract

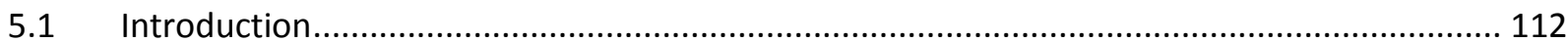

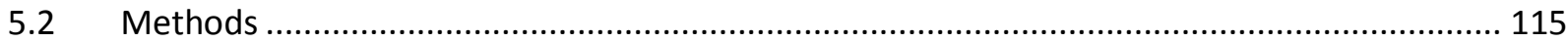

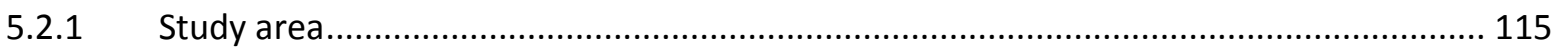

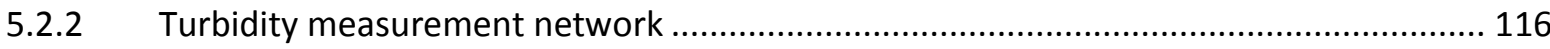

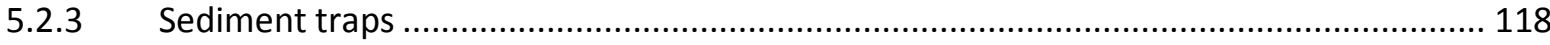

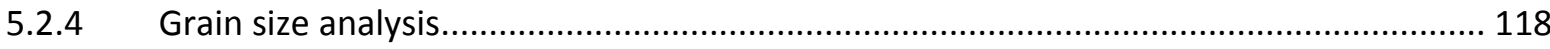

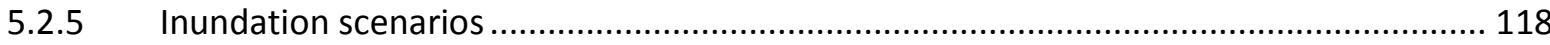

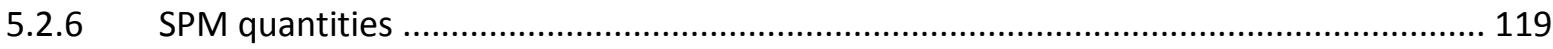

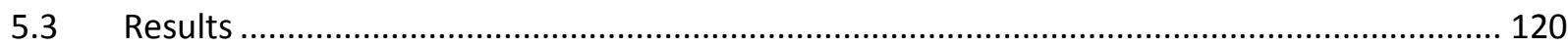

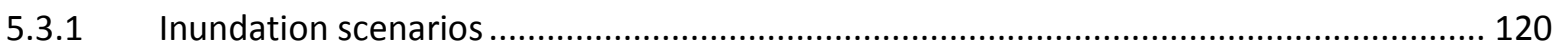

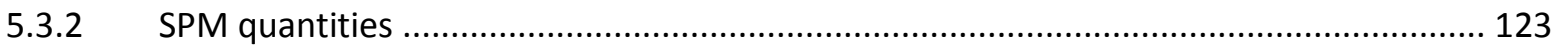

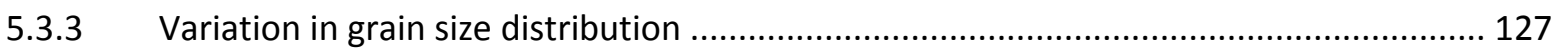

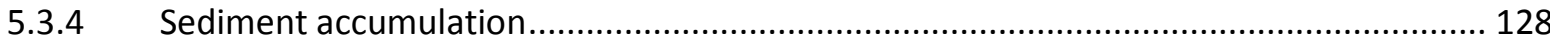

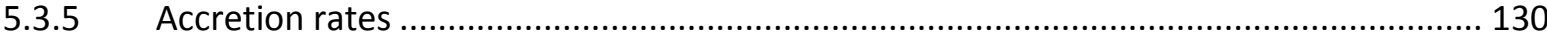

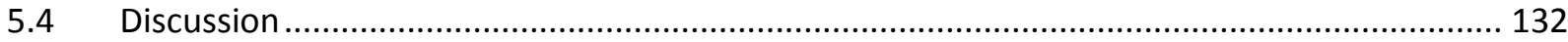

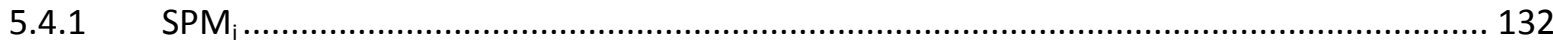

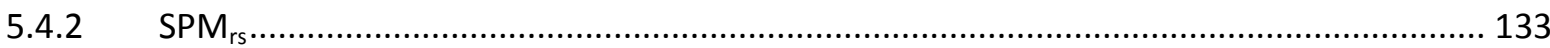

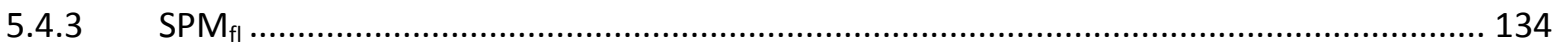

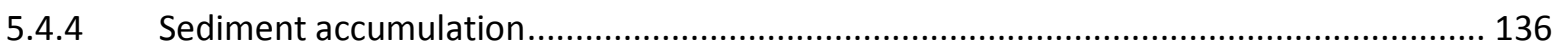

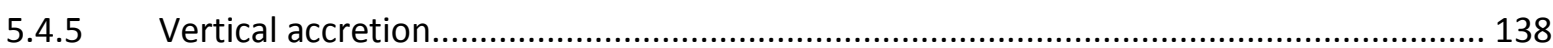

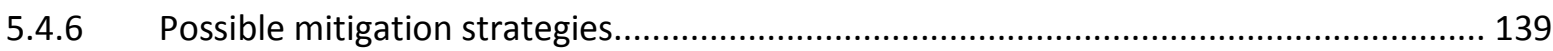

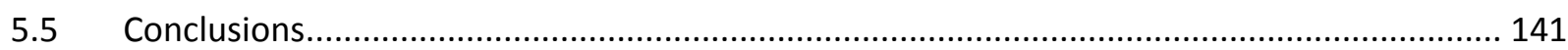

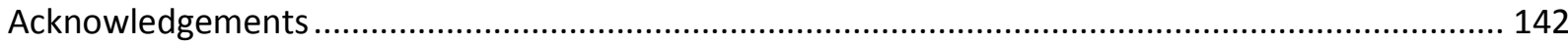

6 Plant traits affect surface elevation change in salt marshes: an example of biogeomorphic

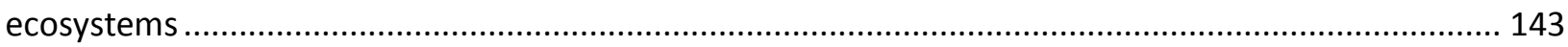

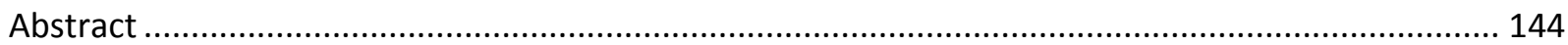

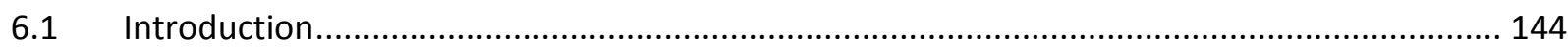

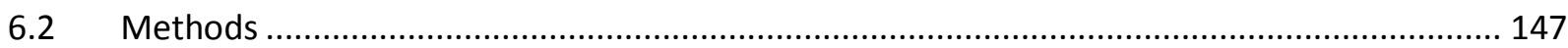

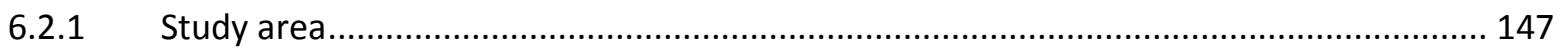

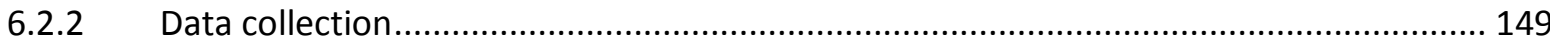

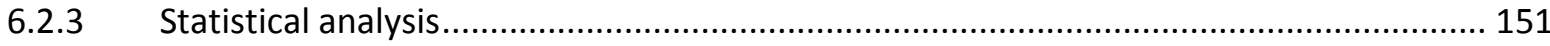

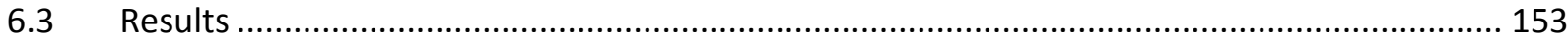

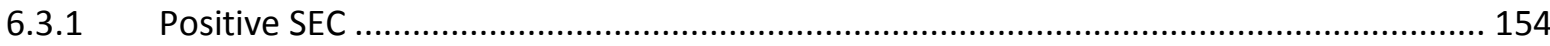




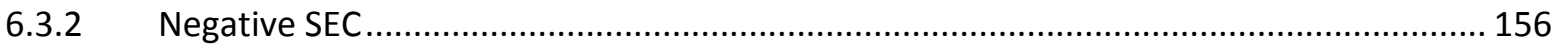

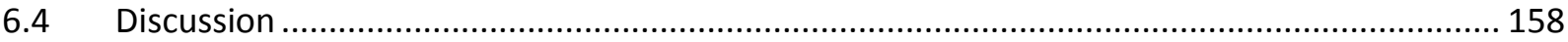

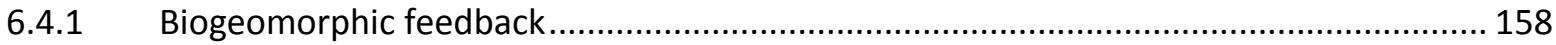

6.4.2 Positive SEC explained by functional traits......................................................... 159

6.4.3 Negative SEC explained by functional traits ....................................................... 160

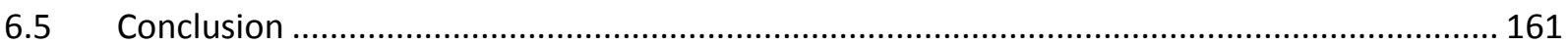

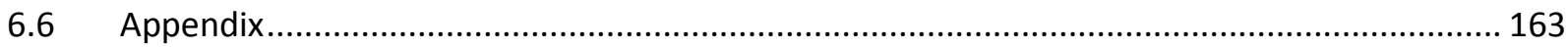

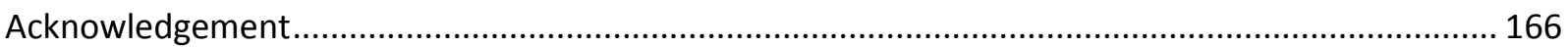

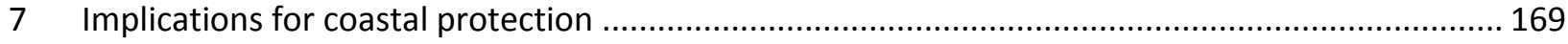

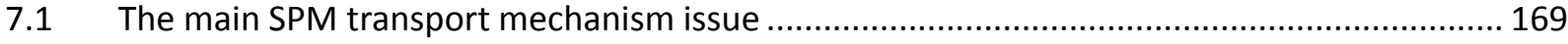

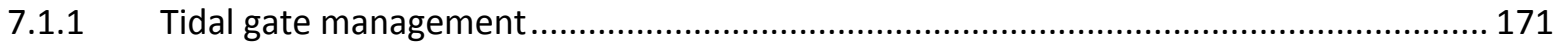

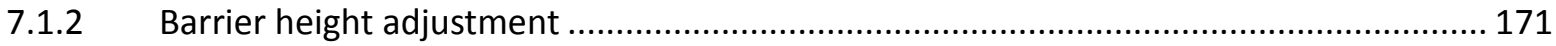

7.2 Theoretical implementation of adjusted protection measures ........................................ 172

7.2.1 Theoretical approach - same flooding frequency ................................................. 172

7.2.2 Theoretical approach - increased flooding frequency............................................. 175

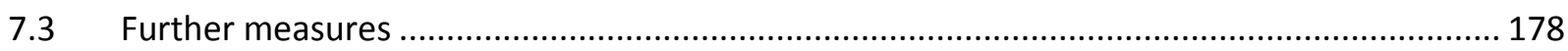

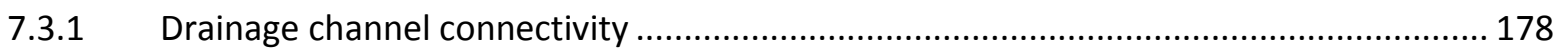

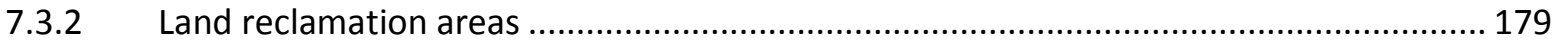

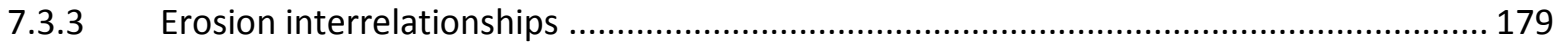

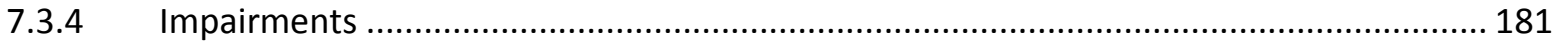

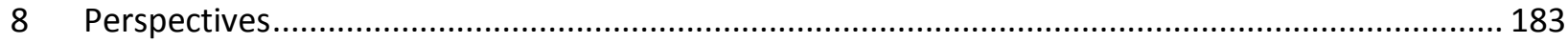

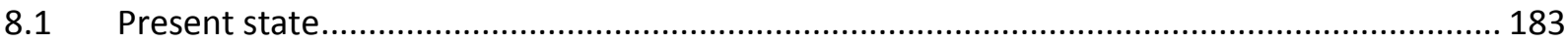

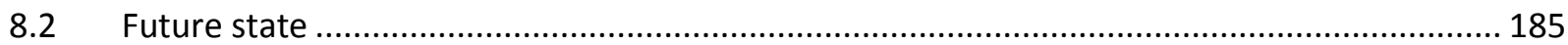

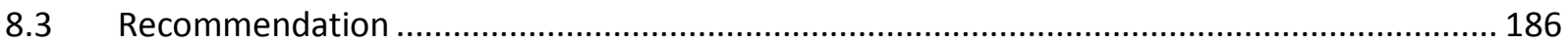

9 Summary

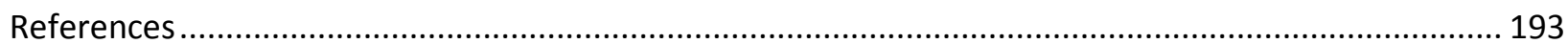

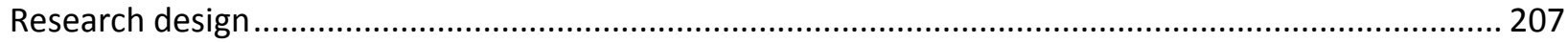

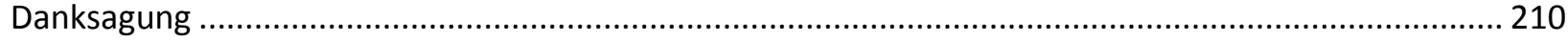




\section{List of Figures}

Figure 1-1: The Wadden Sea between the 15-m depth contour and the embanked marshland and the areal share of land- and sea-scape components in the Wadden Sea region (including the Zuiderzee area in the Netherlands). The blue box contains the area of the North Frisian Wadden Sea. This figure is modified from Reise (2005).

Figure 1-2: The map shows the ten Halligen (green) surrounded by the islands of Föhr, Amrum and Pellworm and the mainland of Schleswig-Holstein (northern Germany) as well as three sand barrier islands. The inset shows the location of the area within the southern North Sea and the neighboring countries: GB = Great Britain, NL = The Netherlands, D = Germany, DK = Denmark, S = Sweden, N = Norway.

Figure 1-3: Impressions of the vegetated landscape of Hallig Langeness showing the flat marshland and dwelling mounts with houses (b) same view as in (a) but during an inundation where only the dwellingmounts stand above the water.

Figure 1-4: View along the Langeness southeastern shoreline showing the tidal flat at low water on the left side with adjacent revetments. On the right side, breakwater constructions are shown with grazing livestock on the marshland surface.

Figure 1-5: A tidal gate in the southeast of Langeness at low water during fair weather conditions (a) and at drainage water flow after a storm surge (b).

Figure 2-1: The location map shows the ten Halligen (dark gray), located in the German Bight. The study area Langeness (dashed outline in black) is surrounded by the islands of Föhr, Amrum and Pellworm and the mainland of Schleswig Holstein as well as several other Halligen and three sand barrier islands. The inset shows the location of the area within the southeastern North Sea and its neighbouring European countries..

Figure 2-2: Digital ground model (based on Laser scan DGM1 data from 2014 courtesy of LKN.SH) of Langeness with the locations of the different components of the autonomous network of turbidity measuring stations and transmission units. The white areas show depths that cannot be detected by laser scanning due to high water coverage. The three white vertical lines are artifacts.

Figure 2-3: Bathymetric map (courtesy of Bundesamt für Seeschifffahrt und Hydrographie) showing the two large tidal channels Norderaue in the north and Süderaue in the south enclosing Langeness. The small pictures show a detailed view of the respective turbidity measurement stations with their neighboring spur dykes in the tidal flats except for station T-6 which is located next to a land reclamation area.

Figure 2-4: Illustration of the turbidity measuring stations: $(A)$ construction of a turbidity measuring station (T-4) located on the tidal flat; (B) entire sensor unit inside the metal grid cage; (C) wiper; (D) OBS3+ sensor. The components: 1 metal post, 2 metal grid cage, 3 POM pressure housing, 4 OBS sensor, 5 wiper, 6 probe, 7 sediment accumulator installed in the field. The topographical survey points of Table 2-1: 8 top, 9 OBS measuring height, 10 grid and 11 ground. 
Figure 2-5: (A) Sketch of a sediment accumulator. Once a particle enters one of the six inlets of the accumulator and is no longer affected by the outer flow conditions, it should sink to the accumulator bottom. (B) Close up of sediment accumulator closed and opened (C).

Figure 2-6: Figure and sketch of the calibration tank. The tank consists of a $1.65 \mathrm{~m}$ long and $0.2 \mathrm{~m}$ wide black PE tube. The sieve (cyan) inside is used for separating the turbulence and the calibration chamber. The pump on the ground is coloured in dark gray. It pumps the suspension within a $0.04 \mathrm{~m}$ wide pipe system (light gray) into the upper turbulence chamber of the tank. The OBS (red) is mounted in the calibration chamber.

Figure 2-7: Calibration results of stations T-1 to T-6 using the accumulator calibration samples (respective median grain size in $[\mu \mathrm{m}]$ in brackets) and the individual incrementally added aliquots (series of black dots for each station). The corresponding linear polynomial fits are shown with dotted lines. The slopes of the regression lines continuously increase with increasing median grain size. The blurred straight lines at the end of the polynomial fit for stations T-5 from $0.38 \mathrm{~g} / \mathrm{l}$ and T- 6 from $0.5 \mathrm{~g} / \mathrm{l}$ indicate the extrapolation range towards higher SPM concentrations. The extrapolation of the calibration curves for these two stations was necessary to cover all measured concentrations from the field, because not enough accumulator sediment was available to extend the range by a further calibration point. The vertical error bars estimates possible deviations of the added aliquot as determined by mean and standard deviation of five aliquots taken from the respective suspension. The horizontal error bars show the standard deviation of the measured OBS signal. The coloured squares show the results of the reference measurements. The inset gives a more detailed view for low OBS response (between 50 and $150 \mathrm{mV}$, grey box).

Figure 2-8: Calibration result of station T-6 with accumulator sediment calibration samples and their individual incrementally added aliquots (series of black dots). Error bars as in Figure 2-7. The corresponding linear polynomial fit is shown with a dotted green line, the $95 \%$ confidence intervals with blue dashed lines and the $99 \%$ confidence intervals with red dashed lines. The green square shows the result of the single reference measurement. The stars show the results of ten in situ samples. One in situ sample is an outlier with $0.24 \mathrm{~g} / \mathrm{I} \mathrm{SPM}$.

Figure 2-9: Frequency distributions of SPM concentrations during two tidal cycles under different weather conditions. The light grey bars show a common tidal cycle under fair weather conditions recorded on November 30, 2017. The dark grey bars show the SPM concentrations during an inundation event within a storm surge in the night of February 11 to 12, 2018.

Figure 2-10: A section of our measured time series shows SPM concentrations for four consecutive tidal cycles for an inundation event in February 2018. Right column shows the measuring duration of the individual stations (vertical coloured bars) in relation to the tidal elevation (tide gauge Hilligenley): T1=black, $\mathrm{T}-2=$ cyan, $\mathrm{T}-3=$ magenta, $\mathrm{T}-4=\mathrm{red}, \mathrm{T}-5=$ blue, $\mathrm{T}-6=$ green. Additional information include, tidal elevation, ten minutes averaged values of wind speeds (circles) and wind directions (crosses) recorded at the weather station Strucklahnungshörn and ten minutes averaged values of significant wave heights from the sea state buoy Süderaue. 36

Figure 2-11: Ten minutes averaged wind speed and direction during the measuring period from October 1, 2017 to February 15, 2018 (winter) and from February 25, 2017 to June 1, 2017 (spring) recorded at the weather station Strucklahnungshörn. The grey box 1 contains the highest wind speeds occurring during the first storm surge event with wind speeds above $20 \mathrm{~m} / \mathrm{s}$ (up to $28 \mathrm{~m} / \mathrm{s}$ ). The grey box 2 
highlight higher maximum wind speeds compared to the winter period. Wind speeds from about northern and SSE directions (box 3 ) are lower compared to the winter months. The colour bar visualizes data density.

Figure 2-12: Density plot of SPM versus wind direction of all stations in the tidal flats measured during the same winter period from October 1, 2017 to February 15, 2018 as in Figure 2-11. The colour bar visualizes data density.

Figure 2-13: Linear regression analysis between wind speed and SPM concentrations, separated for the different wind direction quadrants, during the measured winter period from October 1, 2017 to February $15,2018 . R^{2}$ is the coefficient of determination after Pearson. The four individual inundation events (Table 2-4) are marked with different symbols and colour coding according to the associated wind direction quadrant. Inundation event 3 was not recorded by stations T-3 and T-4 due to technical issues.

Figure 2-14: Density plot of SPM concentrations versus wind direction at stations T-1, T-2, T-3 and T-4 measured during the same spring period as in Figure 2-11, from February 25, 2017 to June 1, 2017.... 42

Figure 2-15: Bar plot of mean SPM concentrations of the measured spring period from 25th February 2017 to 1st June 2017, the winter period from October 1, 2017 to February 15, 2018, and the individual inundation events numbered from 1 to 4 as in Table 2-4. The third inundation event was not recorded at stations T-3 and T-4 due to technical issues.

Figure 3-1: The map shows the ten Halligen (green), located in the North Frisian Wadden Sea in the southern North Sea. The study area Langeness (black dotted outline) is surrounded by the islands of Föhr, Amrum and Pellworm and the mainland of Schleswig Holstein as well as several other Halligen and three sand barrier islands. The inset shows the location of the area within the North Sea and its neighboring countries: GB = Great Britain, NL = The Netherlands, $D=$ Germany, DK = Denmark, $\mathrm{S}=$ Sweden, $\mathrm{N}=$ Norway.

Figure 3-2: (a) Impressions of the vegetated landscape of Langeness showing the flat marshland and dwelling mounts with houses (b) same view as in (a) but during an inundation. (c) Example of the revetment and breakwater construction. (d) The spur dykes enclosing the turbidity measurement station T-2 (circle) in the southwest of Langeness (see Figure 3-3).

Figure 3-3: Digital ground model of Langeness (based on Laser scan DGM1 data from 2014, courtesy of LKN.SH). The black line divides Langeness in its historical parts named Nordmarsch in the west and AltLangeness in the east. The white areas show depths that cannot be detected by laser scanning due to high water coverage in the two tidal channels Norderaue in the north of Langeness and Süderaue in the south. The black roundish areas are the dwelling-mounts.

Figure 3-4: Sketch of a top view trap cluster, consisting of five bottles (trap type A) and five turf mats (trap type B) installed around the cluster center at a distance of approximately five meters. The red inset shows a photo of trap types $A$ and $B$ as installed in the field. Figure $3-4 b$ shows a picture of the turbidity measurement station T-7 on the marshland of Langeness consisting of a pressure housing (1) in which data acquisition and transmission unit is located and the metal grid cage (2) in which the OBS sensor unit is mounted to protect it against flotsam. Next to the OBS sensor unit, 4 sediment accumulators SB (3) are mounted. The stations in the tidal flat have additional sediment accumulators ST in approximately 1.2 meter above the SB accumulators. This construction is surrounded by five sediment 
traps (4). A more detailed description of the functionality (data acquisition and transmission) of the turbidity measurement stations is given in Hache et al. (2019).

Figure 3-5: Linear regression analysis between tidal averaged wind speed and SPM concentrations at three different sites from the turbidity measurement stations T-1, T-2 and T-4 (Figure 3-3), separated for the two main wind direction quadrants from south to west (left) and west to north (right), during both measured winter periods from October 1, 2017 to February 15, 2018 and from September 1, 2018 to March 01, 2019. $R^{2}$ is the adjusted coefficient of determination after Pearson and $n$ is the number of observations. The $p$-values are below the assumed significance level of 0.05 .

Figure 3-6: Left (a, c, e); the tidal averaged development of wind speed and wind direction of five tides that end up in the inundations number 6,5 and 8 within the fifth tide (yellow). The error bars show the standard deviations which indicate tidal averaged wind condition variability. The dashed line shows the average wind speed of $13.3 \mathrm{~m} / \mathrm{s}$ from all 8 inundation cycles and their standard deviation of $3 \mathrm{~m} / \mathrm{s}$ (dotted lines). Right ( $b, d, f)$; the associated tidal averaged SPM for stations in the tidal flat and the average of all stations per tide (av). Inundation number 6 shows the highest average SPM concentration, followed by inundation number 5 and inundation number 8 with the lowest average SPM concentration. Missing tidal averaged SPM at station T-5 and/or T-6 are due to too low water levels during some tides.

Figure 3-7: Overall tidal averaged SPM of the measurement stations in the tidal flat (T-1 to T-6) and averaged Ms accumulation from trap clusters on the marshland for eight sampled inundations in 2017/18 and 2018/19 (Table 3-3).

Figure 3-8: Comparison of the determined tidal averaged SPM on the tidal flat (T-4, T-1) to the respective marshland area $(T-7, T-8)$ for the six individual inundations where water level was high enough to activate at least one of the marshland stations (Table 3-2 and Table 3-3). The ascertained SPM concentrations at station T-4 are compared with the SPM concentrations at station T-7 (red dots) and T-1 with T-8 (black dots), respectively. The number besides the individual dots indicate the number of the inundation. The blue dashed line indicates the highest possible SPM on the marshland based on the SPM availability on the tidal flat.

Figure 3-9: Linear regression analysis between averaged SPM at the two stations on the marshland (T-7 and T-8) and Ms accumulation of traps type A next to both stations for the inundations during the winter season 2018/19 where water level was high enough to activate the respective station. The numbers indicate the number of the inundation (Table 3-3). The regression equation is $y=1.67 * 10-$ $4 * M s+0.0185 . \mathrm{R}^{2}$ is the adjusted coefficient of determination after Pearson and $\mathrm{n}$ is the number of observations.

Figure 3-10: Ms accumulation $\left[\mathrm{g} / \mathrm{m}^{2}\right]$ for all eight inundations (Table 3-3) at three locations $(1,2$ and 3 related to stations T-1, T-2 and T-3; Figure 3-3) with two trap clusters each related to different barrier heights ( $\mathrm{H}=$ high, $\mathrm{L}=$ low, Table $3-4)$.

Figure 3-11: Grain size analysis of samples from the sediment accumulators SB (Base) and ST (Top) at station T-1 and the adjacent trap clusters $1 \mathrm{H}$ and $1 \mathrm{~L}$, for the two inundations number 3 and number 6 (Table 3-3).

Figure 3-12: Median grain size comparison for four inundations number 3, 4, 6, and 8 (3 - circles, 4 squares, 6 - diamonds, 8 - stars) between the sediment accumulators at tidal flat stations T-1 (black) and 
T-2 (cyan), and trap cluster samples from clusters $1 \mathrm{H}, 2 \mathrm{H}$ (unfilled) and $1 \mathrm{~L}, 2 \mathrm{~L}$ (filled). The blue dashed line indicates the 1:1 ratio of median grain sizes in the trap clusters versus sediment accumulator SB (Base, left) or ST (Top, right). For this grain size comparison, only sediment accumulator sample results are shown where it was possible to empty the accumulators immediately before the inundation and sample it as soon as tidal flats were accessible again after the inundation (maximum two days) to minimize potential bias in the sediment accumulators caused by subsequent tides.

Figure 4-1: The map shows the ten Halligen (green). The study area Langeness (black dashed outline) is surrounded by the islands of Föhr, Amrum and Pellworm and the mainland of Schleswig Holstein (northern Germany) as well as nine other Halligen and three sand barrier islands. The inset shows the location of the area within the southeastern North Sea and the neighboring countries: GB = Great Britain, NL = The Netherlands, $\mathrm{D}=$ Germany, $\mathrm{DK}=$ Denmark $\mathrm{S}=$ Sweden, $\mathrm{N}=$ Norway.

Figure 4-2: (a) Hydrodynamic model of the entire North Sea including parts of the North Atlantic by Arns et al. (2015b). The red square shows the location of the high resolution model of the Hallig Langeness (b) including topographic elevation in meter above mean sea level. The magenta dot marks the location of the tide gauge Hilligenley.

Figure 4-3: Close up of the high resolution model of the Hallig Langeness surrounded by 94 sources from which particles are added including topographic elevation in meter above mean sea level.

Figure 4-4: Breakwater height adjustments at Hallig Langeness chosen for three particle track simulation cases ( $A, B$ and $C$ ) showing in meter above mean sea level. The red framed sections in case $B$ and $C$ show the areas where the heights were adjusted to lower values compared to case $A$.

Figure 4-5: Tidal cycles from the hydrodynamic simulations of the moderate inundation (black line) and the storm surge (blue line) which end up in the tidal cycle that inundates Langeness, calculated at the location of the tide gauge Hilligenley (see Figure 4-2b). The dashed red line shows the reference height for water levels from which a storm surge occurs according to the German Federal Maritime and Hydrographic Agency (BSH). The dashed grey line shows the mean breakwater height of Langeness according to Schindler and Willim (2015).

Figure 4-6: Particle class $1(30 \mu \mathrm{m})$ track simulation results of accumulated particles on Langeness including the breakwater heights in meter above mean sea level. The left side shows the moderate inundations from top to bottom ( $A 1, B 1, C 1)$ of all breakwater adjustments $A$ to $C$ in Figure 4-4 and the right side shows the storm surge event in the same order (A2, B2, C2).

Figure 4-7: Bar chart showing particle transport, loss and accumulation quantities in case of $30 \mu \mathrm{m}$ particle size, for moderate inundations (A1, B1, C1) and storm surge simulations (A2 B2 C2), normalized to the particle transport of simulation $A 2$.

Figure 4-8: Bar chart showing particle transport, loss and accumulation quantities in case of $80 \mu \mathrm{m}$ particle size, for moderate inundation (C1) and storm surge simulations (A2 B2 C2), normalized to the particle transport of simulation A2.

Figure 4-9: Linear regression analysis of seven individual modelled inundations (Table 4-2) between simulated peak high water levels and normalized particle accumulation quantities to the particle accumulation of simulation A2 (inundation event 1 in Table 4-2) resulted in a coefficient of 
Figure 4-10: Scatter plot of the difference in the resulting flow velocities on Langeness between storm surge simulation $\mathrm{A} 2$ and $\mathrm{B} 2$ averaged during the flood and ebb phase. The white areas on Langeness show areas where no higher flow velocities occur due to the breakwater height adjustments (see Figure 4-4).

Figure 4-11: Scatter plot of the difference in the resulting flow velocities on Langeness between storm surge simulation $\mathrm{A} 2$ and $\mathrm{C} 2$ averaged during the flood and ebb phase. The white areas on Langeness show areas where no higher flow velocities occur due to the breakwater height adjustments (see Figure 4-4).

Figure 5-1: Map of the ten Halligen (green), located in the North Frisian Wadden Sea in the southeastern North Sea. The study area Langeness (black dashed outline) is surrounded by the islands of Föhr, Amrum and Pellworm and the mainland of Schleswig Holstein as well as the nine other Halligen and three sand barrier islands. The inset shows the location of the area within the North Sea and its neighboring countries: GB = Great Britain, NL = The Netherlands, $D=$ Germany, $D K=$ Denmark, $\mathrm{S}=$ Sweden, $\mathrm{N}=$ Norway.

Figure 5-2: Digital ground model of Langeness (based on Laser scan DGM1 data from 2014, courtesy of LKN.SH) adjusted after Hache et al. (2020). The black line divides Langeness in its historical parts named Nordmarsch in the west and Alt-Langeness in the east. The white areas show depths $>2.4 \mathrm{~m}$ below NHN including the two main tidal channels Norderaue and Süderaue. The black roundish areas on the marsh surface are the dwelling-mounts. Inset 1 shows (from left to right) the tidal flat at low water next to the revetment and breakwater construction. Inset 2 shows a tidal gate at low water in the southeast of Langeness adjacent to turbidity measurement stations T-1 and T-8. The magenta box in the southeast shows the location where the photos were taken for Figure $5-8$.

Figure 5-3: Gauge level data from the tide gauge Hilligenley (blue), the interior tide gauges Kichhofswarf (red) and Kirchwarf (black); a) example of a moderate inundation that occurred on March 05, 2019, b) storm surge SF2 at January 15, 2020 and c) storm surge SF1 at December 15, 2019. Dashed lines show the mean ground level height of $1.53 \mathrm{~m}$ above NHN of Langeness (green), mean height of the water impermeable coastal protection structures of $2.34 \mathrm{~m}$ above NHN (grey; Schindler and Willim 2015) and the reference height for storm surge water levels of $2.92 \mathrm{~m}$ above NHN (cyan). At storm surge SF1 no data was recorded by tide gauge Hilligenley during the ebb phase due to technical issues (c).

Figure 5-4: Comparison of $\mathrm{SPM}_{\mathrm{i}}$ on the tidal flats (T-4, T-1) with $\mathrm{SPM}_{\mathrm{rs}}$ on the marshland at rising water level (T-7, T-8). In addition to data from seven storm surge inundations (squares) SPM values of individual moderate inundations recorded by Hache et al. (2020) are shown (circles). The $\mathrm{SPM}_{\mathrm{i}}$ at station T-4 are compared with SPM ${ }_{r s}$ at station T-7 (west) and SPM at T-1 with SPM $\mathrm{Sm}_{\mathrm{rs}}$ at T-8 (east). Individual storm surge events are labelled according to Table 5-1. The dashed lines show, for orientation, percentages of $\mathrm{SPM}_{\mathrm{i}}$ attained by $\mathrm{SPM}_{\mathrm{r}}$

Figure 5-5: Water level data (upper graph) of tide gauge Hilligenley (blue) and interior tide gauge Kirchwarf (black). The lower graph shows individual SPM concentrations (not averaged) from three stations T-1 (black), T-8 (orange) and T-G (cyan) during the two storm surge induced inundations SF3c and SF3d on February 11 and 12, 2020 (Table 5-1). The black box highlights the SPM concentrations at 
station T-G during the drainage water flow through the tidal gate. The red vertical lines resemble the separation into flood/ebb phase.

Figure 5-6: $\mathrm{SPM}_{\mathrm{rs}}$ versus $\mathrm{SPM}_{\mathrm{fl}}$ for seven storm surge induced inundations. The values at T-7 (dark grey), T-8 (orange) and T-G (cyan) from two different OBS calibrations, coarse grain size calibrated SPM (squares) and the fine grain size-calibrated SPM (triangles). The linear fits refer to the SPM concentrations of station T-7 and T-8. The five circles (light grey) show the dry mass concentration in suspension samples taken as reference. The dashed line indicates the 1:1 ratio between SPM $\mathrm{rs}_{\mathrm{s}}$ and $S P M_{f \mid}$

Figure 5-7: Grain size distribution $(0.4$ to $2000 \mu \mathrm{m})$ of the sediment accumulator samples SB and ST at station T-1 and the adjacent sediment trap samples $1 \mathrm{~L}$ (lower breakwater) and $1 \mathrm{H}$ (higher breakwater) from a moderate inundation (a), storm surge SF2 (b) and storm surge SF1 (c) (Increasing peak water level). The respective median is displayed as circle. The respective samples were collected during one single tidal cycle. The associated tide gauge levels are shown in Figure 5-3 in the same order from (a) to (c).

Figure 5-8: Impressions of the tidal flat to the southeast of Langeness (for location see magenta box in Figure 5-2) during low water before the storm surge SF3 with the revetment and breakwater construction in the background (a). The tidal flat was predominantly covered with fine sand and some silt-sized material on top. (b) shows the same area during low water after the storm surge SF3 but viewed from the top of the breakwater construction towards the tidal flat. The silt-sized material is removed and darker parts in the tidal flat indicate spots where erosion has exposed the peat below. The inset (c) shows an area close to the tidal channel with deep erosion patterns in the tidal flat. 133

Figure 5-9: Linear regression analysis between SPM $\mathrm{rs}_{\mathrm{s}}$ at the two stations on the marshland (T-7 and T-8, red and black lines, respectively) and sediment accumulation of bottle traps next to both stations for all storm surge events and the moderate inundations during the winter season 2018/19 (Hache et al. 2020). Storm surges that could be sampled after one tide are shown with squares. Sampled storm surges that contain sediment accumulation data and SPM $\mathrm{M}_{\mathrm{rs}}$ of at least two or more tides are shown by diamonds. The moderate inundations are shown by circles. Dashed magenta line refers to T-7-8 (all data from T-7 and T-8 except for the summed storm surge SF3a to SF3e from T-7). $R^{2}$ is the adjusted coefficient of determination after Pearson and $\mathrm{n}$ is the number of observations.

Figure 6-1: Map of the North Sea coast of Lower Saxony and Schleswig-Holstein including the islands and Halligen off the mainland coast. The island study area is indicated by a filled triangle, mainland study areas are indicated by filled circles, Hallig study areas are indicated by filled squares. (Single- column fitting image).

Figure 6-2: Annual SEC of the study areas island, mainland (due to regional differences divided between Lower Saxony and Schleswig-Holstein), and Hallig. The boxes represent the interquartile ranges, the whiskers the upper and lower fences, the circles outliers, the thick lines indicate the medians, and the solid dots the means. The dashed line shows the annual mean sea-level rise (3.6 mm/a) in the German Bight from 1971 to 2008 according to Wahl et al. (2011). Letters indicate significant differences among study areas according to a Tukey-HSD post hoc test. (Single-column fitted image).

Figure 6-3: Factors explaining positive SEC on salt marshes of (a) island ( $n=23)$, (b) mainland ( $n=105)$, and (c) Halligen $(n=113)$. Distance to marsh edge is boxcox transformed, where a value of -1 corresponds to 
$77 \mathrm{~m}$, a value of +1 corresponds to $431 \mathrm{~m}$. Canopy height $(\mathrm{CH})$ is $\mathrm{ORQ}$ transformed, where a value of -1 corresponds to $6.8 \mathrm{~cm}$, a value of +1 corresponds to $28.2 \mathrm{~cm}$. 'Shoot mass' values are the scores of the first axis from the PCA (see Appendix Figure 6-5) using LA, LBM, SBM, and SSL. (2-column fitted image).

Figure 6-4: Factors explaining negative SEC on salt marshes of $(a)$ island $(n=15)$ and $(b)$ mainland $(n=79)$ locations. Distance to the marsh edge is ORQ transformed, where a value of -1 corresponds to $125 \mathrm{~m}$, a value of +1 corresponds to $390 \mathrm{~m}$. 'Anchoring' values are the scores of the first axis from the PCA (see Appendix Figure 6-6) using BIBM and SRL. (2-column fitted image)...

Figure 6-5: Two-dimensional illustrations of the standard PCA ordination results of plots that experienced positive SEC. Arrows indicate the direction of loading for each trait. The scores of the first axis are included in the model as 'shoot mass'. LA, leaf area; LBM, leaf biomass; SBM stem biomass; SSL, specific stem length. (Single-column fitted image).

Figure 6-6: Two-dimensional illustration of the standard PCA ordination results of plots that experienced negative SEC. Arrows indicate the direction of loading for each trait. The scores of the first axis are included in the model as 'anchoring'. BIBM, below-ground biomass; SRL, specific root length. (Singlecolumn fitted image).

Figure 7-1: Modified Sketch of the working principle of the controlling flooded polder after Vandenbruwaene et al. (2011), illustrated for a storm surge (a), and for a mean tide during flood (b) and during ebb (c). 170

Figure 7-2: (a) shows the location of the two sediment trap cluster on Langeness (blue filled circles) where the sediment deposits were collected within six years to calculate annual vertical accretion rates. (b) shows the annual vertical accretion rates $(\Delta \mathrm{H})$ related to the total inundation events per year (c) $\Delta \mathrm{H}$ related to total moderate inundations per year and (d) $\Delta \mathrm{H}$ related to total storm surge induced inundations per year. The six-year time series includes annual vertical accretion data from 2010 to 2013 by Schindler et al. (2014a).

Figure 7-3: Digital ground model (based on Laser scan DGM1 data from 2014 courtesy of LKN.SH) showing depths from 1 to 5.9 metres above mean sea level. The $1.43 \mathrm{~m}$ threshold represents the mean ground level of Nordmarsch and $1.62 \mathrm{~m}$ of Alt-Langeness and $2.34 \mathrm{~m}$ represents the mean barrier height of Langeness according to Schindler and Willim (2015). The black line divides Langeness in its historical parts named Nordmarsch in the west and Alt-Langeness in the east. 176 


\section{List of Tables}

Table 2-1: Topographical survey of all turbidity measurement stations in meters above MSL. The exact locations of the four measuring levels from top to ground are illustrated in Figure 2-4.

Table 2-2: Total measurement duration in days from February 25, 2017 to February 15, 2018 and the number of recorded storm surge events within the winter measurement period from October 1, 2017 to February 15, 2018.

Table 2-3: $\mathrm{d} 50$ percentile (median) of bed sediment collected next to each measurement station and Base accumulator sediment samples which we have used for OBS calibration collected at each station over a period of one month from January 8 to February 9, 2018 without occurring storm surge events. The measurements were carried out with a laser particle sizer LS13320 by Beckman\&Coulter.

Table 2-4: Key parameters averaged over four tidal cycles within four different storm surge events leading to inundation of Langeness. Ten minutes averaged mean wind speeds and directions from the weather station Strucklahnungshörn and ten minutes averaged mean significant wave heights from the sea state buoy Süderaue.

Table 3-1: Vertical marshland accretion rates $\Delta \mathrm{H}$ of three sampled winter seasons displayed as three year average of all trap clusters as well as the minimum and maximum values $\Delta \mathrm{h}$. Number of sampled inundations and the mean inundation height based on data from the interior tide gauges Kirchhofswarf (KHW) and Kirchwarf (KW) in m above m.s.l (Figure 3-3).

Table 3-2: Total number of recorded tides within the turbidity measurement network and the total number of recorded tides that lead to an inundation of the entire Hallig according to the inundation criterion (cf. section 2.4) within winter seasons from October 1, 2017 to February 15, and from September 1, 2018 to March 01, 2019. Missing inundation measurements at station T-3 and T-4 were due to technical issues.

Table 3-3: Tidal averaged parameters for eight different inundations measured at Langeness. Ten minutes averaged mean wind speeds and directions from the weather station Strucklahnungshörn (located $22 \mathrm{~km}$ southeast of the tide gauge Hilligenley) and ten minutes averaged mean significant wave heights from the sea state buoy Süderaue. Mean SPM values from the whole turbidity network in the tidal flat (av SPM) and mean $\mathrm{M}_{\mathrm{s}}$ accumulation rates in the trap clusters and mean inundation heights above marsh surface from both interior tide gauges Kirchhofswarf and Kirchwarf.

Table 3-4: Key parameters of all trap cluster pairs with different barrier heights ( $\mathrm{H}=\mathrm{high}$ and $\mathrm{L}=\mathrm{low}$ ). The barrier heights are measured from the seabed of the nearby turbidity measurement station to the top of the barrier (elevation data from GNSS topographical survey of the LKN.SH). The average $M_{s}$ accumulation is the mean of all eight inundations. The average inundation SPM (SPM availability on the tidal flat) is the mean of all tidal averaged SPM of eight inundations measured at the nearby turbidity measurement station on the tidal flat ( $\mathrm{T}-1$ for $1 \mathrm{H}$ and $1 \mathrm{~L}, \mathrm{~T}-2$ for $2 \mathrm{H}$ and $2 \mathrm{~L}, \mathrm{~T}-3$ for $3 \mathrm{H}$ and $3 \mathrm{~L}$ ). 70

Table 4-1: Two particle classes and their used parametrization for the particle tracking 
Table 4-2: Particle accumulation quantities and their ratios $(80 \mu \mathrm{m} / 30 \mu \mathrm{m})$ of all simulation cases from A1 to $\mathrm{C} 2$ from both particle classes.

Table 4-3: All seven simulated inundation events with the two key parameters for the linear regression analysis in Figure 4-9; particle accumulation quantities $(30 \mu \mathrm{m})$ normalized to the particle accumulation of simulation A2 (inundation event 1 ) and peak high water levels. The observed peak high water levels are records from tide gauge Hilligenley except inundation event 1 which was recorded by tide gauge Dagebüll. The inundation events printed in bold are storm surges.

Table 4-4: Inundation durations of the marshland during the flood-and ebb phase and the sum of both phases from the moderate inundation and storm surge simulation cases (see e.g. Figure 4-6) in minutes measured at the location of the interior tide gauge Kirchwarf on Langeness $\left(54.640^{\circ} \mathrm{N}, 8.614^{\circ} \mathrm{E}\right)$. The standard deviation is always \pm 10 minutes due to the chosen evaluation interval. The flood phase contains the duration where water level crosses the mean ground elevation of Langeness ( 1.53 m.a.m.s.l.) according to Schindler and Willim (2015) until peak high water level is reached. The duration of the following ebb phase contains the water levels from peak high water to the highest reduction in the breakwater height to $1.9 \mathrm{~m}$ above mean sea level. This assumption is made because the subsequent drainage water flow below $1.9 \mathrm{~m}$ above mean sea level only takes place through the tidal gates and to avoid potential bias caused by subsequent rising tides especially after the storm surge. 106

Table 5-1: Parameters of seven storm surge induced inundations.

Table 5-2: Median grain sizes of sediment accumulator samples from individual storm surge induced inundations and one moderate inundation that is used for the individual OBS sensor calibration.

Table 5-3: Sediment accumulation from sediment traps (bottles) and SPMrs as average (av) of all measured moderate inundations and storm surges at station T-7, T-8 and from all 12 sediment trap fields (bottles) on the marshland.

Table 5-4: Vertical marshland accretion rates $\Delta \mathrm{H}$ of four sampled winter seasons including minimum and maximum values $\Delta \mathrm{H}$ and the average inundation height.

Table 6-1: Plot locations, number of replicates, and data sources for the three salt marsh types; island, mainland (LS: Lower Saxony), and Halligen. In addition to the sources for vegetation and SEC data, all community-weighted trait data were unpublished. Ntotal = total number of plots of the study area, NPSEC = number of plots which experienced positive SEC, NNSEC = number of plots which experienced negative SEC.

Table 6-2: Dependent and independent (biotic and abiotic) variables. Given are the plant functional traits, the corresponding abbreviations used in this study, as well as the aggregated variables based on a principle-component analysis (if traits were correlated) that are included in the multiple linear regression. Given are also minimum and maximum values, units and applied transformations; ORQ, ordered quantile normalization transformation.

Table 6-3: Results of the multiple linear regression model and stepwise variable selection for positive SEC on island marshes, mainland marshes and Hallig marshes. Given are estimates, standard error, tvalues, and significance levels. Significant $p$-values at $\alpha<0.05$ are shown in bold type. In addition, measures of model performance are shown. 
Table 6-4: Results of the multiple linear regression model for negative SEC on island- and mainland marshes. Given are estimates, standard errors, $t$-values, and significance levels. Significant $p$-values at $\alpha$ $<0.05$ are shown in bold type. In addition, measures of model performance are shown.

Table 6-5: Functional traits and the aggregated variable 'shoot mass' of species growing on plots that experienced positive SEC. CH, canopy height $(\mathrm{cm})$; LA, leaf area $(\mathrm{mm} 2)$; LBM, leaf biomass $(\mathrm{mg})$, SBM, stem biomass (mg); SSL, specific stem length $(\mathrm{mm} / \mathrm{mg})$.

Table 6-6: Functional traits and the aggregated variable 'anchoring' of species growing on plots that experienced negative SEC. BIBM, below-ground biomass (mg); SRL, specific root length $(\mathrm{mm} / \mathrm{mg})$. .... 166

Table 7-1: Case A shows calculated annual vertical marshland accretion rates within six sampled years and as a six-year average ( 6 yr. av) of both sediment trap clusters (see Figure 7-2) as well as the total number of moderate inundations and storm surges and their six-year average. Same vertical accretion calculation but without SPM loss (A w. loss), or with adjusted southern barrier heights (C), or in combination by preventing SPM loss with adjusted southern barrier heights (C. w. loss).

Table 7-2: Case A shows calculated annual vertical marshland accretion rates within three sampled years and as a three year average ( $3 \mathrm{yr}$. av) of all 12 trap clusters. Same calculation but without SPM loss (Case A w. loss), or with adjusted southern barrier heights (C), or in combination by preventing SPM loss with adjusted southern barrier heights (C. w. loss).

Table 7-3: Flooding frequency in total and its rounded annual average in brackets within the sampling period from October 2017 to March 2020. The additional floods are caused by the barrier height adjustment. Flooding beside season indicates the number of floods beside the annual storm season which last from October to March. Case $\mathrm{C}$ refers to the southern barrier height adjustment in Chapter 4. 


\section{ABBREVIATIONS}

AIC Akaike Information Criterion

A.M.S.L. Above Mean Sea Level

AV

Average

BIBM Below-Ground Biomass

$\mathrm{CH} \quad$ Canopy Height

CWM Community-Weighted Mean

DGM Digital Ground Model

ECMWF European Centre for Medium-Range Weather Forecasts

FM Flexible Mesh

GPS Global Positioning System

GSD Grain Size Distribution

IPCC Intergovernmental Panel on Climate Change

ISM-Band Industrial, Scientific and Medical Band

LA Leaf Area

LBM Leaf Biomass

LOI Loss On Ignition

LSU Livestock Unit

MHW Mean High Water

MSL Mean Sea Level

OBS Optical Backscatter Sensor

PCA Principal-Component Analysis

POM Polyoxymethylene

PSD Particle Size Distribution

PT Particle Tracking

RCP Representative Concentration Pathway

RMSL Relative Mean Sea Level

SB Sediment Accumulator Base

SBM Stem Biomass

SEC Surface Elevation Change

SLR Sea Level Rise

SPM Suspended Particulate Matter

SRL Specific Root Length

SSL Specific Stem Length

ST Sediment Accumulator Top

UNESCO United Nations Educational Scientific and Cultural Organization 


\begin{abstract}
Inhabited coastal areas around the world have to deal with sea level rise (SLR) and its likely consequences such as increasing natural hazards like heavy storms, flooding events and coastal lowland loss by drowning over the long-term. The latter threat also affects the so-called Halligen, ten island-like marsh areas in the North Frisian Wadden Sea (southern North Sea), Germany. Like marshes in general, periodic inundations of the Halligen have the potential to mitigate or even hamper the risk of drowning by sedimentation and subsequent surface elevation increase. This natural adaptation capacity is strongly influenced by the availability of suspended sediments in the intertidal flats and the transportation of sediment on supratidal flats where it can settle. Coastal protection measures like dykes and breakwater constructions were implemented in the past to prevent erosion of the Hallig margin but are nowadays suspected to limit the sediment transport towards the marsh surface. The result is that vertical accretion of the supratidal areas is outpaced by SLR. Assessment of suspended sediment availability in tidal flat systems and transport mechanisms is therefore necessary to determine the possible adaptation capacity of the marshland against present and future SLR. The latest research results show that today's accretion rates on the Halligen are out of balance with present SLR. Lack of knowledge about suspended sediment quantities makes it impossible to answer the main question, whether a limited suspended sediment availability in the intertidal flats or an inefficient sediment transport towards the marsh surface is responsible for the imbalance. The development and usage of an autonomously working turbidity measurement network based on IR backscatter sensors and accompanied by suspended sediment accumulators and sediment traps is central to this thesis and to the framework of the project "LivingCoastLab". The availability of suspended sediment and its transport around and on the Hallig Langeness is measured and assessed during numerous events that range from moderate to storm surge induced inundations. These measurements revealed insights into strong and systematic suspended sediment availability and its increasing as well as decreasing controlling factors. Furthermore, these measurements revealed insights into sediment transport limitations caused by coastal protection measures and demonstrated the high potential of storm surges in generating vertical accretion rates capable of compensating SLR. However, this high potential is not fully utilized as large sediment quantities are lost before they can increase vertical accretion rates due to a disadvantageous coastal protection management. A detailed
\end{abstract}


hydrodynamic model of Hallig Langeness and surrounding tidal flats is used to investigate the transport of suspended sediment during inundations and to simulate various scenarios with adapted coastal protection measure heights at the Hallig margin. The results quantitatively show the potential to increase the accretion rates by decreasing the transport limiting effects of the coastal protection measures. Furthermore, the positive and negative effects on vertical accretion rates of functional plant traits are investigated in a collaborative study. Finally, a theoretical approach revealed an almost doubling of today's vertical accretion rates where coastal protection measure heights are decreased and the potential of storm surges are fully utilized. Consequently, the results of these studies lead the way to mitigate the impact of future SLR on the North Frisian Halligen. 


\section{Introduction to the thesis}

\subsection{Motivation}

Roughly estimated, more than 200 million coastal inhabitants already live five meters below mean sea level worldwide and this number is estimated to rise to about 400 to 500 million by the end of the 21st century (WOR1 2010). This is an concerning development as the Intergovernmental Panel on Climate Change (IPCC) projects a global mean sea level rise (SLR) based on Representative Concentration Pathways (RCP) scenarios between 4-9 (RCP2.6) and 10-20 (RCP8.5) mm per year by the end of this century (Oppenheimer et al. 2019). SLR and likely consequences such as increasing coastal flooding (Seneviratne et al. 2012), increase in the frequency of extreme events (Hunter 2009) and higher storm surge water levels (Arns et al. 2015a) thus will affect the coastal regions and their inhabitants around the world. Blankespoor et al. (2014) quantified the impact of a 1-m sea-level rise on coastal wetlands in 86 developing countries and territories. The latter authors found that approximately $68 \%$ of coastal wetlands in these countries are at risk with a large percentage in Europe and Central Asia, East Asia, and the Pacific, as well as in the Middle East and North Africa. These consequences are particularly evident for low lying tidal marsh areas which are part of vegetated coastal habitats that are ranked among the most ecologically productive and economically valuable ecosystems on Earth (Costanza et al. 1997). They provide habitat and regulate nutrient fluxes (Barbier et al. 2011), act as net sinks of carbon and have a great potential for becoming long term carbon storage (Artigas et al. 2015; Peck et al. 2020) which makes them climatically significant. Furthermore, tidal marsh areas show their value as an important component of coastal protection schemes in dissipating wave energy during storm surges (Möller et al. 2014). Protection and utilization of such marsh areas seems highly relevant when facing the assumption that 20 to $50 \%$ may be drowned by SLR until the end of this century (e.g. McFadden et al. 2007; Craft et al. 2009). As todays intertidal zones are mostly limited in extent due to flood defenses such as revetments, dykes, and embankments to protect today's populated and vulnerable coastlines (Temmerman et al. 2013), a SLR driven onshore migration of marsh areas is thus limited in space. This finally results in "coastal squeeze" (van der Wal and Pye 2004) where onshore migration is not possible, feedbacks between tidal flooding, plant growth, and sediment transport allow 
marshes to adapt to a wide range of relative SLR rates in the vertical dimension (Kirwan and Megonigal 2013; Kirwan et al. 2016b). Consequently, the availability of suspended sediment on intertidal flats and its transport towards the supratidal marshland where it can subsequently settle is essential to generate vertical accretion rates to keep pace with SLR. High SLR rates and low sediment supply rates, however, are at least partially responsible for recent marshland decline (Kirwan and Murray 2008). These circumstances are also particularly evident for the socalled Halligen, ten anthropogenic island-like marshlands located in a highly dynamic tidal flat in the southern North Sea, Germany. Recent research findings reveal that today's vertical accretion rates of the Halligen are out of balance with local SLR (Schindler et al. 2014a) which indicates a limited sediment supply. Hence, the main question arises: are today's imbalance between vertical accretion rates and SLR of these anthropogenic marshlands driven by a material limitation (i.e. there is not sufficient sediment available in the water column around the Halligen) or by a transport limitation (i.e. sediment is available but not transported efficiently onto the Halligen)? This Ph.D.-thesis will contribute to answer that question by assessing the spatial and temporal distribution of suspended sediment around and on the largest Hallig Langeness. The implementation of the assessment takes place with a combination consisting of field measurements by using a unique autonomous working turbidity measurement network and numerical model calculations with a detailed 2D hydrodynamicnumerical model from Langeness and the surrounding tidal flats. These suspended sediment investigations cover a large number of inundation events during various weather scenarios including fair weather to storm surge conditions. Based on these investigations and the large obtained dataset resulted in additional insights into sediment dynamics on the marshland. These insights offer approaches for new coastal protection strategies and a sustainable longterm management to mitigate the imbalance between vertical accretion rates and SLR on the unique Halligen.

\subsection{Regional setting}

According to Reise et al. (2010), the Wadden Sea (Figure 1-1) emerged about 8000 years ago and evolved along the North Sea shore and include today the largest coherent tidal flat area of the temperate world consisting of vast bare sand and mud flats, emerging twice daily at low tide. Nowadays, a mainland coastal plain of about $24000 \mathrm{~km}^{2}$ comprises $15000 \mathrm{~km}^{2}$ of 
embanked marshes and embayments (including the former Zuiderzee, Netherlands), $1000 \mathrm{~km}^{2}$ of islands and high sandy shoals, $200 \mathrm{~km}^{2}$ of salt marshes along the mainland coast and $100 \mathrm{~km}^{2}$ on the islands, $4000 \mathrm{~km}^{2}$ of intertidal sand and mud flats, and also $4000 \mathrm{~km}^{2}$ of a sandy subtidal zone dissected by deep tidal channels and inlets (Reise 2005).

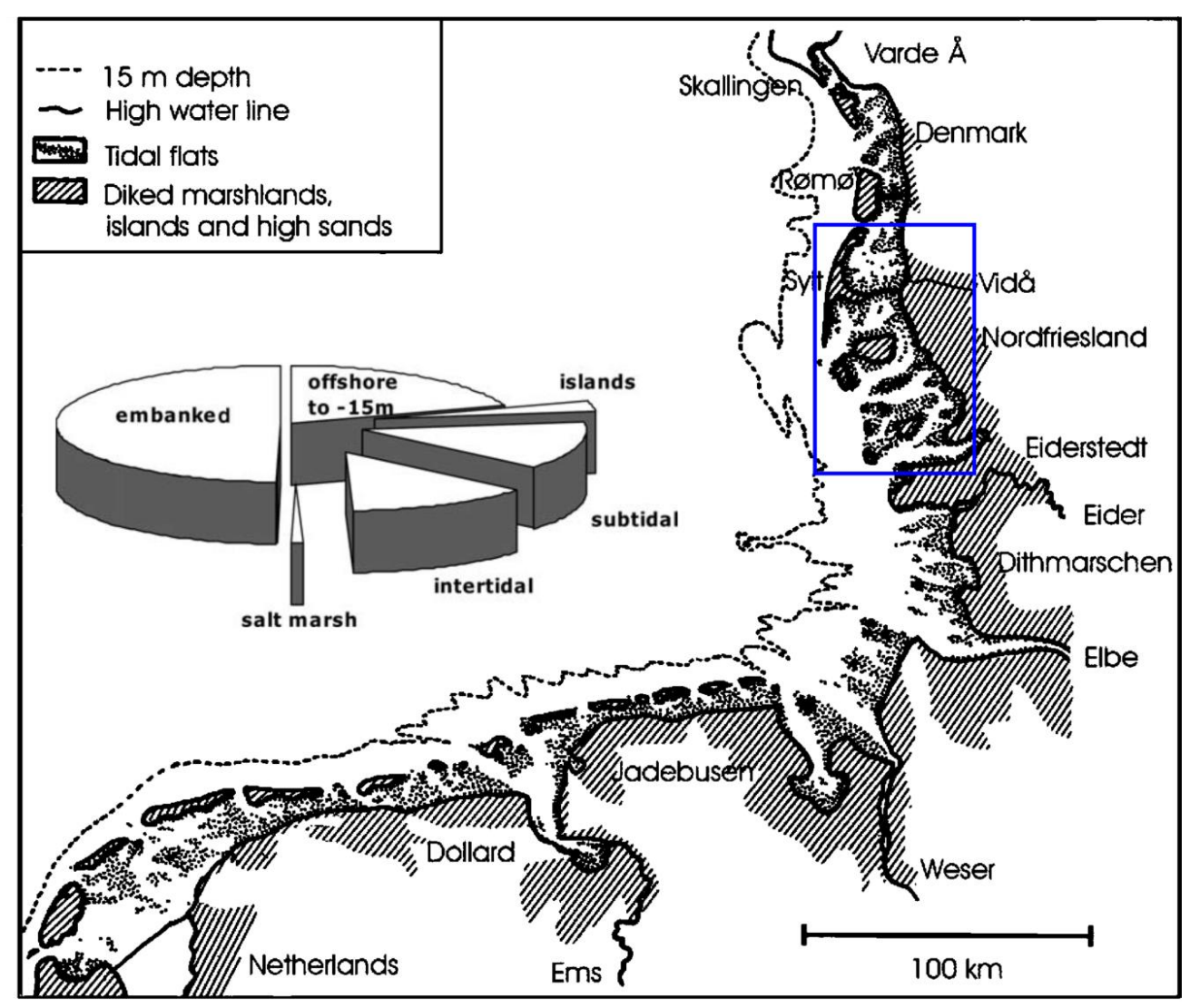

Figure 1-1: The Wadden Sea between the 15-m depth contour and the embanked marshland and the areal share of land- and sea-scape components in the Wadden Sea region (including the Zuiderzee area in the Netherlands). The blue box contains the area of the North Frisian Wadden Sea. This figure is modified from Reise (2005).

The world's largest unbroken intertidal sand and mud flats was a main reason for UNESCO to declare the Wadden Sea as World Natural Heritage Site (Hofstede and Stock 2018). The salt marshes of the Wadden Sea make up about $20 \%$ of the total area of salt marshes along the European Atlantic and Baltic coasts (Wolff et al. 2010). In general, the tidal flats and salt marshes function as wave dissipation zone (Möller et al. 2014). This is an important aspect as the wave dissipation zone decreased hydrodynamic forcing on the mainland flood defence structures during storm surges (e.g. Hofstede 2015) that significantly influence the safety of at least 3.5 million inhabitants of the whole Wadden Sea region (CPSL 2010).

The North Frisian Wadden Sea (see blue box in Figure 1-1) is located the coastline of the federal state of Schleswig-Holstein, Germany and is part of the national park Schleswig- 
Holsteinisches Wattenmeer. This part of the Wadden Sea contains the North Frisian Islands and ten island-like marsh areas, the so called Halligen (Figure 1-2). The North Frisian Halligen are recognized as a UNESCO biosphere reserve and bird protection and flora-fauna habitat area of the European Union. Adjacent to the Halligen, three sand barrier islands (Figure 1-2) dissipates most of the energy of the incoming deep-water waves from the North Sea and are therefore important for the long term stability of the southern part of the North Frisian Wadden Sea (Hofstede 1997). Hence, this set of Halligen and barrier islands thus fulfil an important aspect in mitigating the hydrodynamic forces during extreme weather conditions before it can put the mainland coastline under pressure (Hofstede 1999).

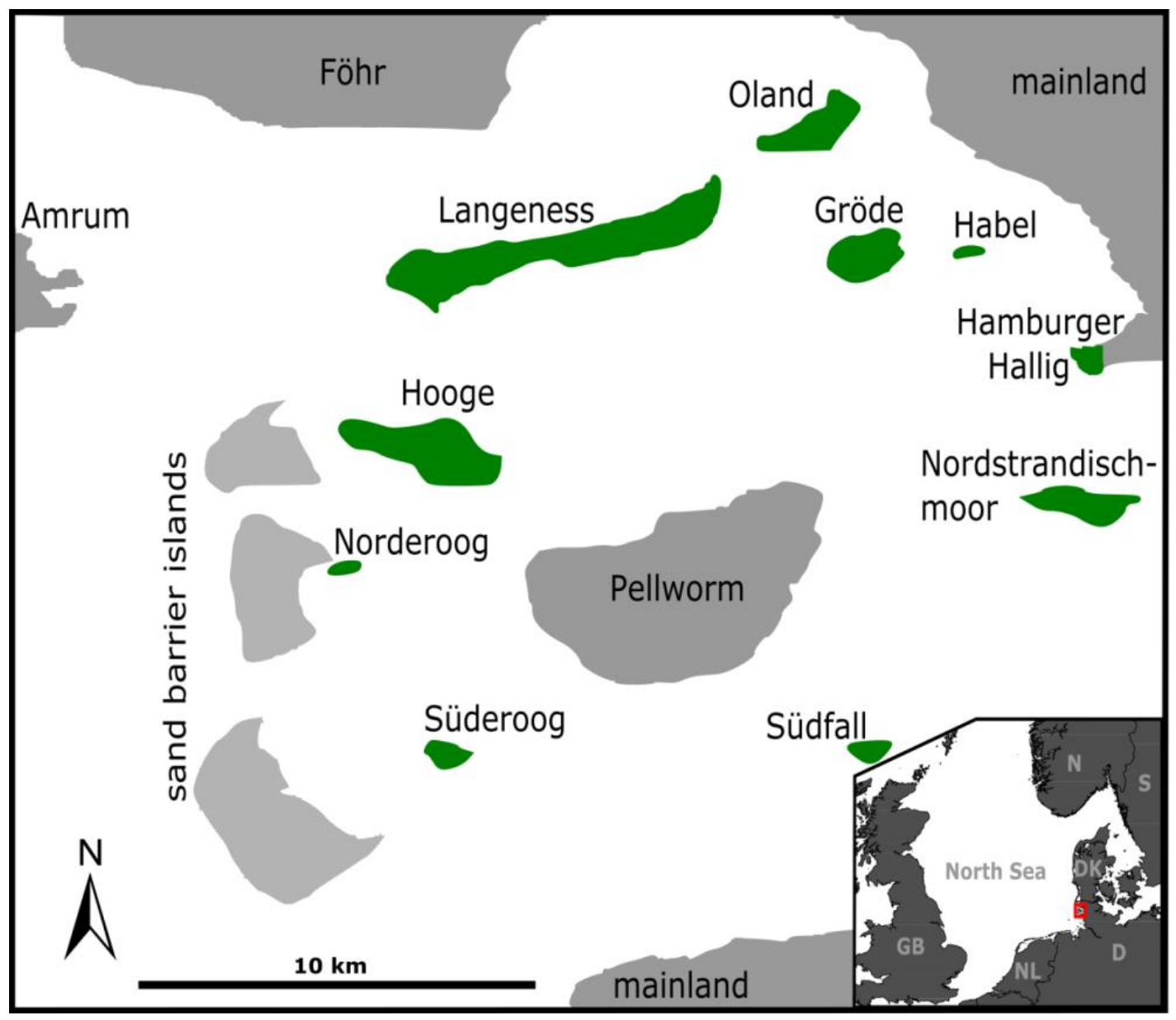

Figure 1-2: The map shows the ten Halligen (green) surrounded by the islands of Föhr, Amrum and Pellworm and the mainland of Schleswig-Holstein (northern Germany) as well as three sand barrier islands. The inset shows the location of the area within the southern North Sea and the neighboring countries: GB = Great Britain, NL = The Netherlands, D = Germany, DK = Denmark, S = Sweden, N = Norway.

The Halligen have a total area of approximately $23 \mathrm{~km}^{2}$ and an average elevation of only a few decimetres above the mean high water (MHW). Unlike other North Frisian Islands the Halligen consists of Holocene marine marsh sediments rather than Pleistocene glacial deposits which 
make them more prone to erosion. The so-called 1st and 2nd Grote Mandränke were, in 1362 and 1634, respectively, the most catastrophic storm surge events in historical time, where thousands of people lost their lives. The latter storm surge created the last huge land loss in the North Frisian Wadden Sea (Reise 2005) and gave the Halligen their current isolated appearance (Ahrendt 2007) as remnants of the formerly coherent marshlands. Nevertheless, the remaining Halligen have lost between 50 and 80 percent of their land mass (on average) since the first survey in 1713 until the authorities started to stabilize the Hallig margins around 1900 (Quedens 1991). These protective measures are still important because the Halligen are constantly affected by increasing SLR, extreme weather conditions and storm surges (Deicke et al. 2007) that are able to inundate the entire Halligen (Figure 1-3).

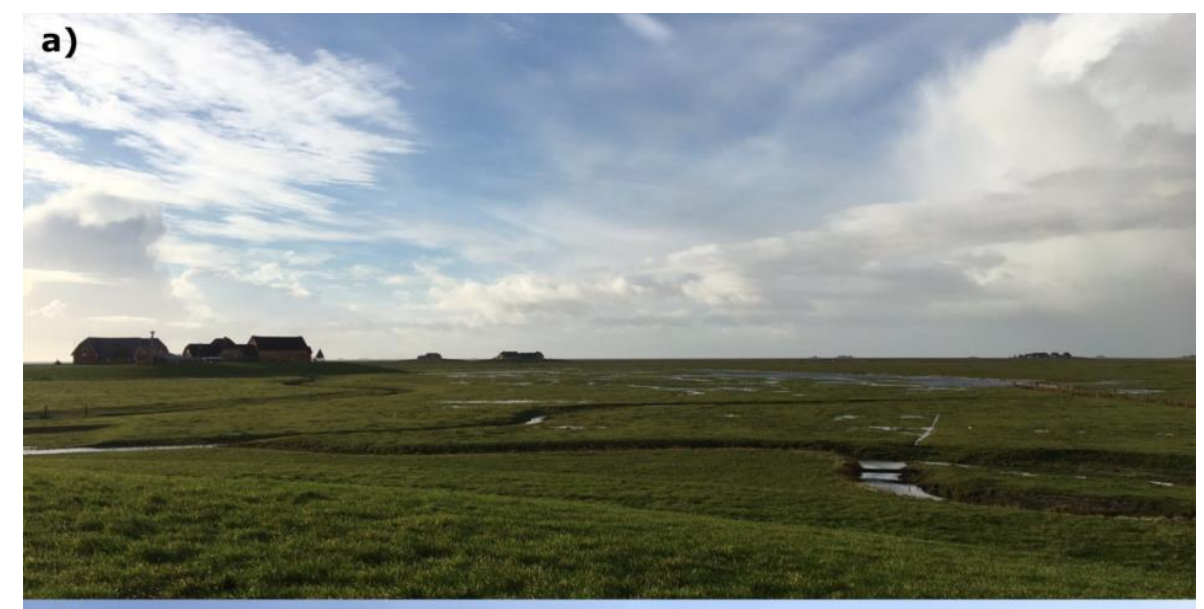

b)

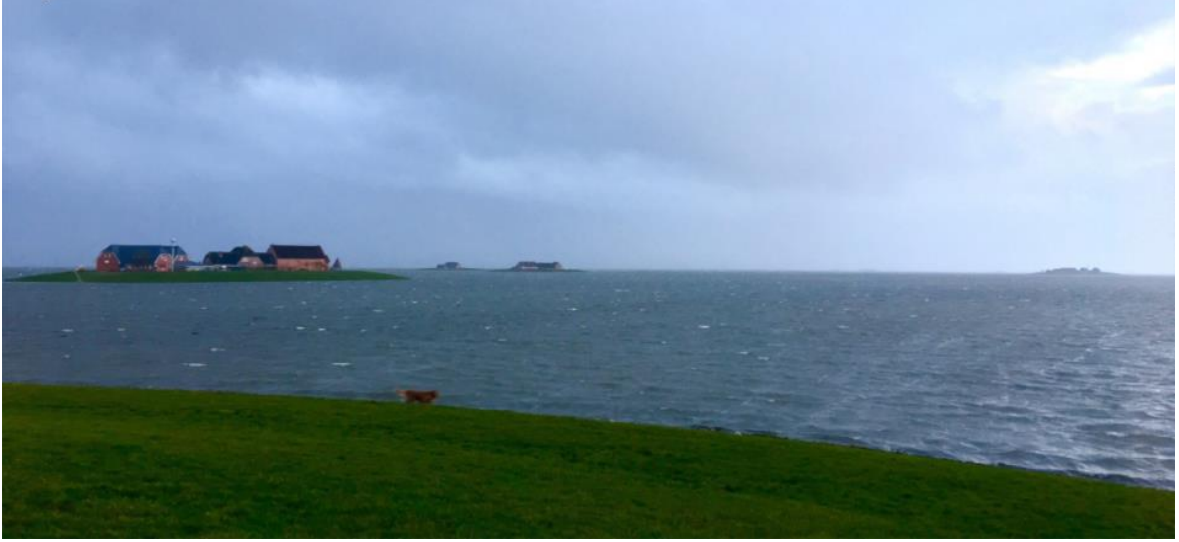

Figure 1-3: Impressions of the vegetated landscape of Hallig Langeness showing the flat marshland and dwelling mounts with houses (b) same view as in (a) but during an inundation where only the dwellingmounts stand above the water.

These extreme weather conditions are common in the winter months and are usually caused by strong westerly winds superimposing on high astronomical tides. The approximately 300 inhabitants of the Halligen protect themselves, their cattle and goods against these inundations by living on man-made dwelling-mounts (called "Warfen"). These Warfen were elevated in 
2000 and further increases are being implemented due to the continuous SLR rise (Hofstede 2019). Protection measures like dykes and revetments encompass the larger Halligen to protect them from erosion and minor inundations. These protection measures are especially relevant during the summer season when farmers cultivate their fields and graze livestock while migratory birds rest and breed. Breakwater constructions were partially installed along the Hallig edge to reduce erosion by wave impact at higher water levels (Figure 1-4). After an inundation, tidal gates ensure a fast drainage of the marshland (Figure 1-5). Spur dykes have been installed to keep the tidal channels at some distance from the Hallig edge to protect the marshland against flow induced erosion.

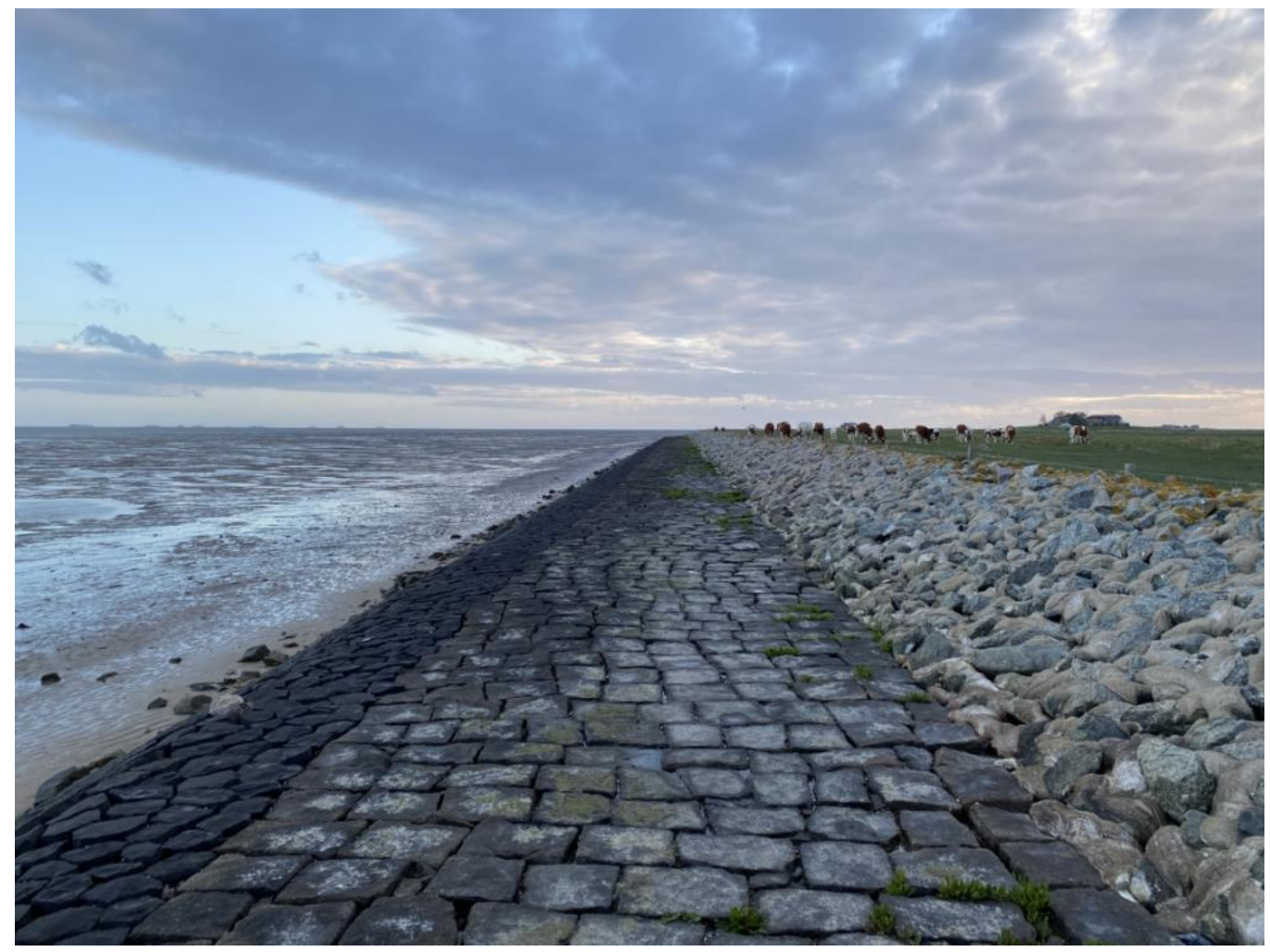

Figure 1-4: View along the Langeness southeastern shoreline showing the tidal flat at low water on the left side with adjacent revetments. On the right side, breakwater constructions are shown with grazing livestock on the marshland surface. 


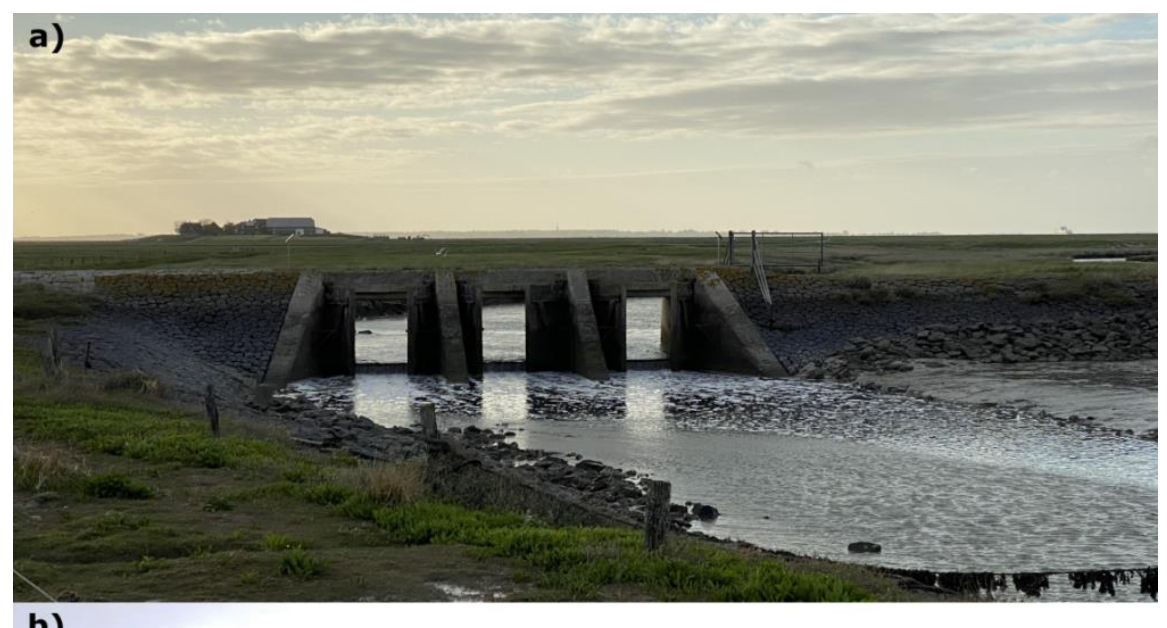

b)

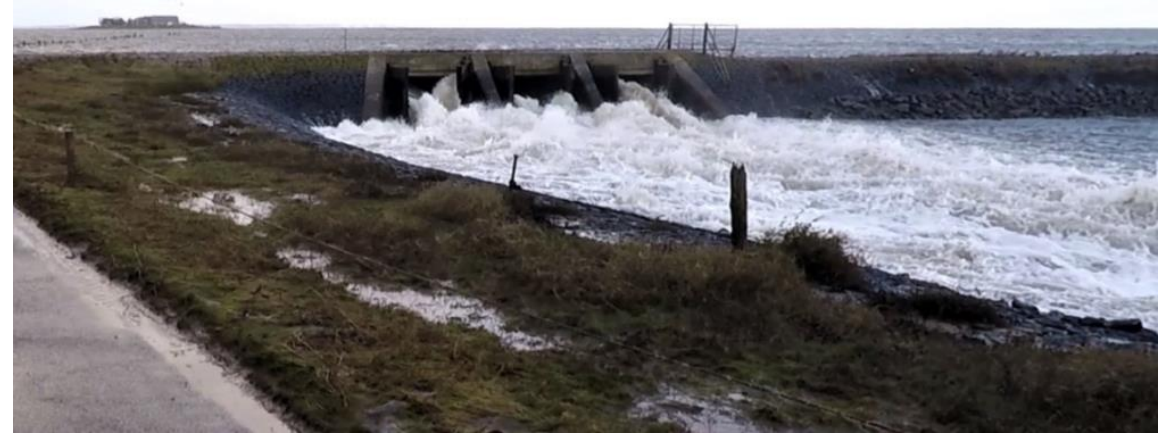

Figure 1-5: A tidal gate in the southeast of Langeness at low water during fair weather conditions (a) and at drainage water flow after a storm surge (b).

\subsection{State of research}

Global and regional SLR affects the conservation and natural adaptation capacity of intertidal and especially supratidal areas which is why the topic of protection and utilization of these areas has become a major research issue (Kirwan et al. 2010, 2016a, 2016b; Kirwan and Megonigal 2013; D'Alpaos et al. 2011; Schuerch et al. 2012, 2013; Horton et al. 2018). The measurement of suspension load, i.e. suspended particulate matter (SPM), represents a major tool to understand sediment origin, transport and dispersal which is why turbidity measurements with optical systems became a fundamental method during the last decades (e.g. Downing 2006). Until today the assessment of solids in suspension is still a challenging task as no standardized method exists to calibrate the measuring devices properly for any given environment. The specific characteristics and interferences that superimpose the responded signal vary widely within each study area which are dependent on particle size, shape, surface 
roughness, the degree of flocculation (e.g. Agrawal et al. 2008; Verney et al. 2009), air bubbles, biological matter (Bunt et al. 1999) and the colour of the sediment (Sutherland et al. 2000). Making SPM assessments was (e.g. Sternberg et al. 1986; Downing and Beach 1989; Kineke and Sternberg 1992; Black and Rosenberg 1994; Bunt et al. 1999) and is still a challenging task (e.g. Druine et al. 2018; Fettweis et al. 2019; Hache et al. 2019), but one that is necessary to determine SPM availability and its transport. New ways to investigate the sediment transport are hydrodynamic models with the use of particle tracking which has become a state-of-the-art method. This method was already used to investigate sediment deposition pattern in the Rhine river by Thonon et al. (2007), by Allison et al. (2017) to track particle paths in the Mississippi Delta and their accumulation spots towards the Gulf of Mexico, by Liubartseva et al. (2018) to examine drifted and accumulated plastic particles in the Mediterranean Sea and by Stanev et al. (2019) to investigate the propagation pathways of marine litter in the southern North Sea. Hence, a combination of SPM measurements and numerical modelling can deepen our understanding of sediment availability and transport mechanisms. These mechanisms are becoming highly relevant for low-lying tidal marsh areas as sediment availability and its transport are indispensable for their natural adaptation capacity. Each tidal cycle that inundates the marsh surface provides SPM that can accumulate on the marsh surface which leads to a vertical accretion of the marsh surface (e.g. Schuerch et al. 2012; Schindler et al. 2014a). The quantities of SPM for supplying natural marshes with sediment depends mainly on the flooding frequency and proximity to the SPM source (Stoddart et al. 1989), tidal variations (Temmerman et al. 2003a, 2003b), the availability of SPM and the rate of SLR (e.g. Temmerman et al. 2004; Andersen et al. 2011). Human interventions such as dykes, sea walls and embankments as well as their continuous heightening and widening to keep up with SLR can block the wave- and wind-driven supply of sediments along coastlines (e.g. Temmerman et al. 2013) which would increase the limitations in adaptation to SLR. To predict the anthropogenic modified Halligens adaptation capacity to recent and future SLR, Schindler et al. (2014b) developed a field and laboratory method to calculate marshland accretion rates based on short-term measurements of sediment depositions on Hallig Hooge, Langeness and Nordstrandischmoor. The results revealed vertical accretion rates ranging from $1.5 \pm 0.9 \mathrm{~mm} /$ a for Hallig Hooge, $1.2 \pm 0.8 \mathrm{~mm} / \mathrm{a}$ for Langeness and $2.6 \pm 0.9$ for Nordstrandischmoor which are out of balance with the local mean high water rise of $5.0 \mathrm{~mm} / \mathrm{a}$ (Schindler et al. 2014a). The latter authors even expected an increased hazard potential for the Halligen due to SLR if vertical marshland accretion does not accelerate in the future. They suggested that a long term persistence of the Halligen can be 
ensured if inundation frequency will increase by deconstruction of dykes and deconstructed tidal channels. Whether these measures promise the desired success of long-term persistence of the Halligen depends mainly on the knowledge of a sufficient SPM availability on intertidal flats during inundation events and its utilization for the supratidal marshland. To this goal, the results of this Ph.D.-thesis will contribute knowledge gained by turbidity measurements and Lagrangian particle tracking during individual inundation scenarios ranging from moderate inundations to storm surges. The findings allow new long-term strategies to be developed for a sustainable ecosystem management with focus on maintaining resilience as considered by e.g. Scheffer et al. (2001) and Hofstede (2019).

\subsection{Thesis Outline}

Chapter 1 introduces the Ph.D.-thesis and highlights the need of the investigated topic.

Chapter 2 represents the development and application of an autonomous working turbidity measurement network, which was installed around and on the largest Hallig Langeness. This chapter explains in detail the functionality (data acquisition and transmission) and the calibration of the turbidity measurement network. Furthermore, first insights into SPM variabilities around Langeness within different time scales, ranging from single tides up to several months within a spring (from February 25, 2017 to June 1, 2017) and a winter (from October 1, 2017 to February 15, 2018), are provided as a time series. The time series included several storm events and four inundations which allows a direct SPM concentration comparison between fair and harsh weather conditions. To highlight the impact on weather conditions on SPM concentrations, gauge level-, wind- and wave data were post processed and evaluated.

In Chapter 3, eight individual moderate inundations are analyzed separately which occurred in the first two winter measuring seasons between December 2017 and March 2019. Beside the evaluation of the turbidity-, gauge level, wind- and wave data, we evaluated sediment accumulation on marshland and suspended sediment samples on tidal flats. The sediment accumulation samples were prepared to quantify total mass of solids, as well as clastic and organic fractions to calculate vertical accretion rates. In addition, laser granulometry was applied to measure the grain size distribution of all collected sediment samples. This variety of data enables the assessment of the SPM availability on tidal flats and the transported SPM 
towards the marsh surface during moderate inundations which reveals the SPM transportlimiting effect of coastal protection measures. Furthermore, to deepen our knowledge about the wind driven dependency on generating SPM availability, a correlation between SPM concentrations and wind data on tidal scales was carried out.

In Chapter 4, a detailed hydrodynamic model of the Hallig Langeness and its surrounding tidal flats allows analyses of adjustments on today's coastal protection measures and its impact on sediment transport conditions by using particle tracking of two particle classes $(30 \mu \mathrm{m}$ and 80 $\mu \mathrm{m})$. The analyses included different hydrodynamic scenarios ranging from moderate sea level conditions to storm surge conditions. Furthermore, the model calculations in Chapter 4 highlight an increased particle transport with increasing water level. The model calculations additionally offer insights into disadvantages by inappropriately adjusting the protection measure heights and firstly reveal quantities of transported particles during a storm surge that do not settle or are remobilized and washed off the Hallig, the so-called particle loss.

Chapter $\mathbf{5}$ analyses seven individual inundations caused by storm surges that occurred in the last winter measuring season $2019 / 20$. The analysis is carried out in the same way as the moderate inundations in Chapter 3 to fulfil the understanding of today's possible SPM availabilities and transport SPM conditions in the study area. The evaluations of the storm surges show their importance in generating vertical accretion rates which are able to compensate local SLR. Furthermore, the particle loss from Chapter 4 could be confirmed by measuring and assessing proportions of SPM on the marshland that do not settle or are remobilized and washed off the Hallig. This loss of SPM highlights the relevance to improve the use of storm surges with adapted coastal management strategies to generate higher vertical accretion rates than today. The additional vertical accretion increase that would result if the coastal management strategies were adapted is shown by a theoretical approach.

Chapter 6 analyzes the effects of functional plant traits (e.g. above-ground roughness and below-ground anchoring capacity) and distance to marsh edge on vertical accretion rates on three different marsh types (island salt marsh, mainland salt marsh, Hallig salt marsh). All marsh types showed mean vertical accretion rates below observed local SLR. It is demonstrated that higher roughness supported sedimentation and stronger anchoring capacity can mitigate erosion. In addition, an assumption for adapted vegetation on the Halligen was made to increase vertical accretion rates. 
In Chapter 7, the status quo of the Halligen's issue in gaining high vertical accretion rates despite the presented adaptation strategies is demonstrated. A comparison of a six-year data series of accretion rates within a theoretical approach clarifies the achievements and the limitations of generating accretion rates that compensate SLR. In addition, further adjustments are discussed that have the potential to increase sediment accumulation as well as the erosion potential. Possible side effects of the adjusted measures concerning erosion processes and the impairment of the inhabitant's lifestyle are discussed as well.

Chapter 8 gives present and future perspectives about the assessed SPM availability and its utilization in view of the present and postulated future climate change scenarios in the Wadden Sea. Finally, a recommendation for an implementation of the discussed adaptation measures is given.

Chapter 9 summarizes the Ph.D.-thesis.

Chapters 2 to 6 are identical with the following publications:

\section{Chapter 2}

"The development and application of an autonomous working turbidity measurement network: Assessing the spatial and temporal distribution of suspended particulate matter on tidal flats in the North Frisian Wadden Sea" by Ingo Hache, Volker Karius, Jörg Gutkuhn and Hilmar von Eynatten

Published 2019 in Continental Shelf Research, Volume 176, 36-50

Doi: https://doi.org/10.1016/i.csr.2019.02.010

\section{Chapter 3}

"Suspended particulate matter for sediment accumulation on inundated anthropogenic marshland in the southern North Sea - Potential, thresholds and limitations" by Ingo Hache, Volker Karius and Hilmar von Eynatten

Published 2020 in Continental Shelf Research, Volume 207, 1-14

Doi: https://doi.org/10.1016/i.csr.2020.104214 


\section{Chapter 4}

"Assessing sediment accumulation at inundated anthropogenic marshland in the southeastern North Sea: using particle tracking on modified coastal protection structures" by Ingo Hache, Sebastian Niehüser, Volker Karius, Arne Arns and Hilmar von Eynatten

Published 2021 in Ocean \& Coastal Management, Volume 208, 1-13

Doi: https://doi.org/10.1016/i.ocecoaman.2021.105631

\section{Chapter 5}

"Storm surge induced sediment accumulation on marsh islands in the southeastern North Sea: Implications for coastal protection" by Ingo Hache, Volker Karius and Hilmar von Eynatten

Submitted 2021 to Estuarine, Coastal and Shelf Science

\section{Chapter 6}

"Plant traits affect surface elevation change in salt marshes: an example of biogeomorphic ecosystems" by Julia Bass, Dirk Granse, Ingo Hache, Kai Jensen, Volker Karius, Vanessa Minden, Martin Stock, Sigrid Suchrow and Michael Kleyer

In preparation 


\section{The development and application of an autonomous working turbidity measurement network: Assessing the spatial and temporal distribution of suspended particulate matter on tidal flats in the North Frisian Wadden Sea}

This paper is published 2019 in Continental Shelf Research, 176, 36-50

Doi: https://doi.org/10.1016/i.csr.2019.02.010

Ingo Hache ${ }^{1}$, Volker Karius ${ }^{1}$, Jörg Gutkuhn ${ }^{2}$, Hilmar von Eynatten ${ }^{1}$

Corresponding author: Ingo Hache, University of Göttingen, Geoscience Center, Goldschmidtstr.

3, 37077 Göttingen, Germany, ingo.hache@uni-goettingen.de

${ }^{1}$ Geoscience Center University of Göttingen, Department of Sedimentology and Environmental Geology, Goldschmidtstraße 3, 37077 Göttingen, Germany

${ }^{2}$ ARGUS Gesellschaft für Umweltmeßtechnik mbH, Goethestrasse 35, 27721 Ritterhude, Germany

Keywords: Suspended particulate matter, Turbidity, Langeness, Hallig, Storm surge, Inundation 


\section{Abstract}

Measuring suspension load (i.e. turbidity) in tide-dominated shallow marine systems like the North-Frisian Wadden Sea forms a major challenge with respect to understanding and modelling sediment origin, transport and dispersal. Sediment transport and deposition in highly dynamic and vulnerable coastal regions becomes especially relevant in terms of the natural adaption capacity of intertidal and supratidal areas to global sea-level rise.

We have developed a network of eight autonomously operating turbidity measuring stations in an island-like salt marsh area (Hallig Langeness) in the tidal flats of the North-Frisian Wadden Sea. Using this system we got first insights about the availability of suspended particulate matter (SPM). In order to obtain the best possible estimation of the SPM concentrations in our study area, each measuring station has been individually calibrated considering the local conditions by using sediment collected by self-built sediment accumulators which are installed next to the respective measuring station. With this method we have investigated the availability of SPM and its spatial and temporal distribution during several spring and winter months.

The results indicate strong and systematic SPM variability between individual stations ranging on average from 0.04 to $0.56 \mathrm{~g} / \mathrm{l}$. Control factors include installation altitude, the proximity to tidal channels, the positive influence on SPM availability by adjacent land reclamation areas or the negative influence on SPM availability due to a shielded position between two spur dykes. Further results show a strong increase of the SPM concentration under harsh weather conditions and inundation events whereby strong winds alone are not sufficient for a significant SPM increase. Instead, the combination of suitable wind direction, its strength and duration play a major role. The increase in SPM concentrations under harsh weather conditions tends to be highest at stations with less protection against the open water. Our new extensive network of turbidity measurements also allows for detecting areas with predominantly low SPM availabilities, the so-called low spots. 


\section{$2.1 \quad$ Introduction}

The natural adaption capacity of intertidal and especially supratidal areas to global and regional sea-level rise is a significant research question related to the protection and utilization of flat coastal areas (e.g. Kirwan et al. 2010; Kirwan et al. 2016a; Kirwan et al. 2016b; D'Alpaos et al. 2011; Schindler et al. 2014a; Schindler et al. 2014b). Especially coastal regions that are not protected by dikes react sensitive to changing water levels. Among these regions are the highly dynamic and vulnerable tidal flats within the southeastern North Sea, including the so-called Halligen, ten small island-like salt marshlands within the North-Frisian Wadden Sea (Figure 2-1).

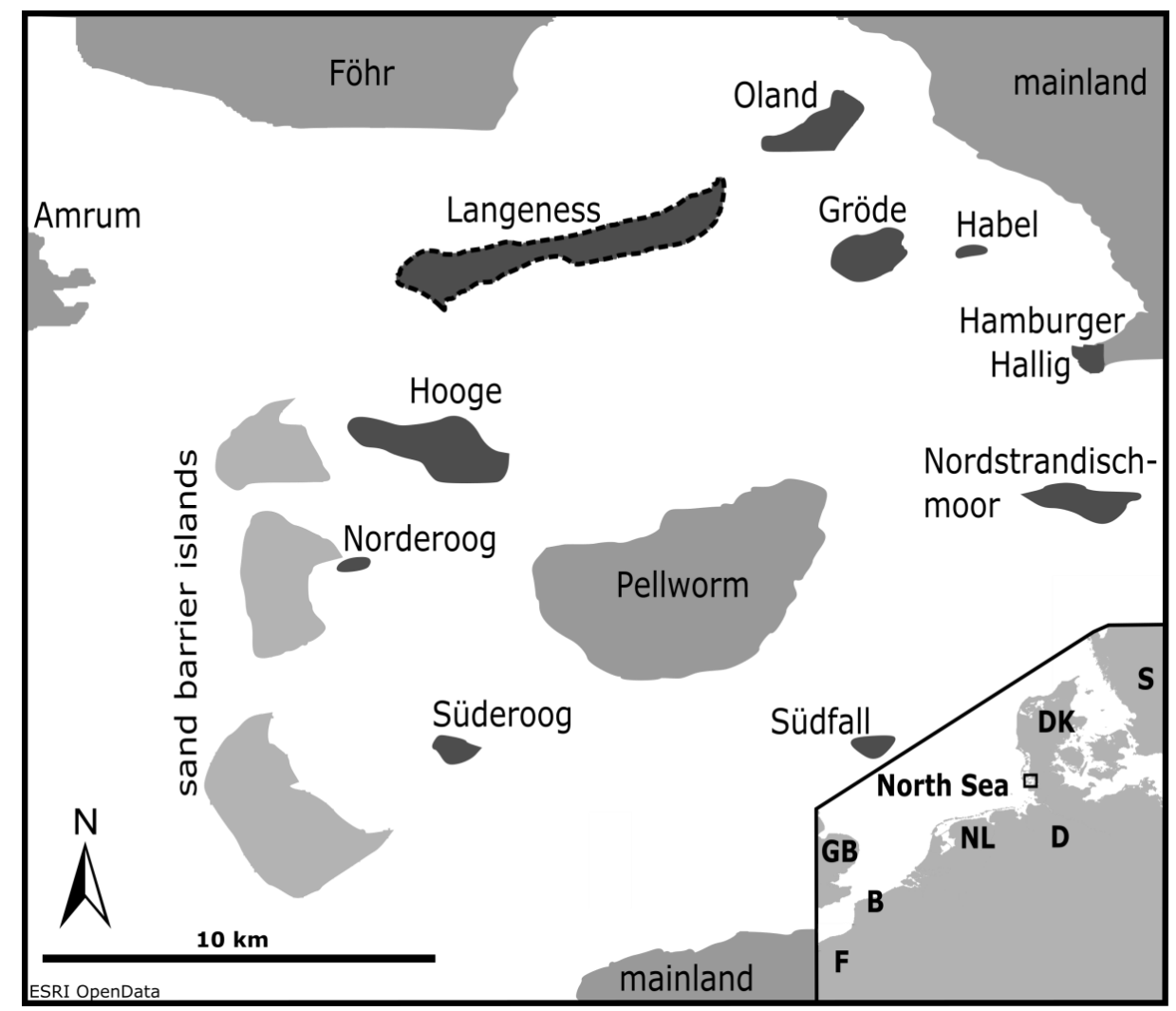

Figure 2-1: The location map shows the ten Halligen (dark gray), located in the German Bight. The study area Langeness (dashed outline in black) is surrounded by the islands of Föhr, Amrum and Pellworm and the mainland of Schleswig Holstein as well as several other Halligen and three sand barrier islands. The inset shows the location of the area within the southeastern North Sea and its neighbouring European countries.

Average elevation of these unique areas is only a few decimeter above the mean high water (MHW). Thus, these areas are particularly affected by an increasing sea-level and by extreme weather conditions (e.g. Deicke et al. 2007, Schindler et al, 2014b). For this reason, protection measures have been taken since centuries to secure the Halligen against erosion and 
inundations. In 1914 low dyke constructions were built on the two largest Halligen Hooge and Langeness (Müller and Fischer 1917), which are supposed to impede inundations of the marsh areas during the summer months. In the following, the rise in sea level and strong floods called for more intensive protection measures in the form of more or less water impermeable revetments and tidal gates to cope with the increasing threat. Some of these measures, however, hamper the long-term self-preservation potential by hindering inundations that would have provided sediments and thus vertical accretion of the land surface (e.g. Schuerch et al. 2013). In view of the rising sea level, however, the support of natural sedimentation and vertical accretion processes are important to counteract the sea-level rise (SLR). At present the prevailing sediment accretion rates on three Nord Frisian Halligen are $1.5 \pm 0.9 \mathrm{~mm} / \mathrm{a}$ for Hooge, $2.6 \pm 0.9 \mathrm{~mm} / \mathrm{a}$ for Nordstrandischmoor and $1.2 \pm 0.8 \mathrm{~mm} / \mathrm{a}$ for Langeness (Schindler et al. 2014a). Longer term accretion rates based on $137 \mathrm{Cs}$ and $210 \mathrm{~Pb}$ datings range from 1.0 to 3.2 $\mathrm{mm} / \mathrm{a}$ (Schindler et al. 2014b). Although these values vary considerably, none of them reaches the rate of the annual average relative mean sea level rise (RMSL) of $4.6 \pm 0.8 \mathrm{~mm} / \mathrm{a}$ determined from linear trend analysis of the tidal gauge at Wyk at the southeast on the island of Föhr (Jensen et al. 2011). Hydrodynamic modelling results for the North Sea based on transient simulations from 1961 to 2100 indicate serious changes in the case of persistent anthropogenic climate change (Gaslikova et al. 2013). Actually without considering SLR in the North Sea, a small increase in extreme surge heights towards the coast of the German Bight is expected, along with even stronger changes along the North Frisian Islands. Moreover, model based investigations of 65 extreme storm events between 1970 and 2009 show that an added rise of $+0.54 \mathrm{~m}$ in mean sea level (MSL) has a dramatic local storm surge water level increase of up to $+0.15 \mathrm{~m}$ in addition to the increased MSL in the shallow water areas of the Wadden Sea (Arns et al. 2015a). It is becoming more and more probable that such a high MSL rise will occur until the end of this century, as recent studies by Nerem et al. (2018) postulate a global sea-level rise of $0.65 \pm 0.12 \mathrm{~m}$ until 2100, which is in agreement with the Intergovernmental Panel on Climate Change (IPCC) 5th Assessment Report (AR5) model projections. The combination of low accretion rates and a continuous SLR calls for a long-term strategy to mitigate the imbalance between SLR and vertical accretion. Developing such a strategy for the protection and preservation of Schleswig-Holsteins Halligen is the main objective of the interdisciplinary joint research project named "Living Coast Lab Halligen". The availability of suspended particulate matter (SPM) in our study area forms a key factor for the natural adaptability of the Halligen to SLR. Considering this, it is of major concern whether the sediment accretion of the Halligen 
during inundations is material-limited (i.e. there is not sufficient SPM available in the water column around the Halligen) or transport-limited (i.e. SPM is available but not transported efficiently onto the Halligen). The latter includes the question if and how the coastal protection measures hinder sediment transport. The Lower Saxony Wadden Sea is a national park and the nature conservation within a national park should aim at allowing natural processes to proceed undisturbed under prevailing natural dynamics (Hofstede and Stock 2018). If not, this could give strong arguments to change coastal protection strategies in the future.

Many efforts have been made to measure turbidity to get representative information about the SPM and its transport in tidal areas, for example in tidal creeks (e.g. Davidson-Arnott et al. 2002; Voulgaris and Meyers 2004), nearshore in the Belgian North Sea (e.g. Baeye et al. 2011) and in the German Bight. Studies concerning the German Bight show that SPM variation in deep water e.g. in tidal channels is mainly controlled by the tidal current. (e.g. Ricklefs 1998; Albers and Lieberman 2007). Apart from tidal current, wave action is another factor that influences SPM especially in shallow water e.g. tidal flats (e.g. Gätje and Reise 1998; Bartholomä et al. 2009). Water samples taken one meter above the seabed within the main tidal channels of the Dithmarschen Bight (German North Sea) revealed sediment concentration variations ranging from $0.04 \mathrm{~g} / \mathrm{l}$ to $1.1 \mathrm{~g} / \mathrm{l}$ and maximum computed depth-averaged values of $0.55 \mathrm{~g} / \mathrm{l}$ (Poerbandono and Mayerle 2005). Further strong tidal current influenced SPM variations ranging from $0.002 \mathrm{~g} / \mathrm{l}$ up to $0.16 \mathrm{~g} / \mathrm{l}$ were observed in the tidal inlet between the East Frisian islands Spiekeroog and Langeoog (e.g. Badewien et al. 2009). In most of these studies, SPM was measured at single stations or from a moving vessel. Consequently, the spatial distribution of SPM in a wider area is difficult to assess. Therefore, we developed and installed an autonomous turbidity measurement network with six stations on the tidal flats around the largest of the Halligen, Langeness and two additional stations on the Hallig itself (Figure 2-2) to collect turbidity data simultaneously and continuously during an ongoing measuring campaign, which started on February 25, 2017 and is scheduled to an end in March 2020.

For each study area to be examined for turbidity has its own specific characteristics and interferences that superimpose the response signal of the optical backscatter sensor (OBS), such as particle size, shape and surface roughness, the degree of flocculation (e.g. Verney et al. 2009), air bubbles, biological matter (Bunt et al. 1999) and even the colour of the sediment (e.g. Sutherland et al. 2000). It has to be emphasized that the response of an OBS is both directly 
proportional to the particle volume concentration and inversely proportional to the particle diameter (Fugate and Friedrichs 2002). Thus, the strongest influence on the OBS response, consequently the most important factor (Down and Lehr 2005) and the biggest source of error for the estimation of the SPM concentration is the variability in particle size distribution (PSD) (e.g. Downing 2006), especially regarding particle size changes below $100 \mu \mathrm{m}$ (Conner and Visser 1992). Because of the infrared radiation scatter-sensitivity of the OBS response on the amount of particles and their PSD, the OBS should be calibrated with sediment from the field site (e.g. Sternberg et al. 1986; Downing and Beach 1989; Kineke and Sternberg 1992). We therefore decided to use sediment from the water column collected separately at all six stations in the tidal flat over a period of one month (08.01. to 09.02.2018). With this method, we can avoid the use of bed samples for OBS calibration since the PSD of bed samples is likely different from the sediments in suspension (e.g. Kineke and Sternberg 1992; Black and Rosenberg 1994 ; Bunt et al. 1999).

In this paper we describe the design of the measuring network, the conditions of data acquisition and transmission and the calibration of the turbidity sensors in the tidal flats. Selected time series collected from the six tidal flat stations are presented. These time series include the comparison of SPM of a single tidal cycle under normal and storm conditions and observations of SPM variability during inundation events. In the winter period 2017/2018 four storm surges leading to inundation of Hallig Langeness could be recorded, which are separately investigated. In addition, seasonal differences due to contrasting meteorological conditions recorded in two time series encompassing 183 tidal cycles in spring and 265 tidal cycles in winter are presented.

\subsection{Material and methods}

\subsubsection{Study area}

Langeness is the largest of the ten presently existing North Frisian Halligen with a total area of $9.24 \mathrm{~km}^{2}$. It is located in the Nord Frisian Wadden Sea off the coast of the federal state of Schleswig-Holstein, Germany (Figure 2-1). It has a population of 113 inhabitants (2018), who live on man-made dwelling-mounts (called "Warfen"). The current altitudes of the dwellingmounts are on average 5.06 \pm 0.45 meter above MSL. The last heightening of the dwellingmounts was in the year 2000 in order to compensate for the rising sea level and further 
increases are planned to ensure the best possible protection of the inhabitants, their cattle and goods against annually occurring storm surge events. The term storm surge is used here to describe a local event associated with harsh weather conditions leading to an inundation of Langeness.

The tidal flats around Langeness differ in bathymetry (Figure 2-2). The areas in the north are higher than those in the west and south. The biggest difference in altitude between the two lowest stations (T-2, T-4) and the highest one (T-6) in the tidal flats is about 1.9 meter (Table 2-1). The different bathymetrical conditions of the tidal flats mirror the position of the two large tidal channels, Norderaue in the north and Süderaue in the south, enclosing Langeness (Figure 2-3). The terrain heights of Langeness have an average absolute ground level without anthropogenic structures of $0.17 \mathrm{~m}$ above MHW (Schindler and Willim 2015). Dykes and revetments increase this mean value to $0.98 \mathrm{~m}$. The western terrain of Langeness is on average about $0.2 \mathrm{~m}$ lower than the eastern part.

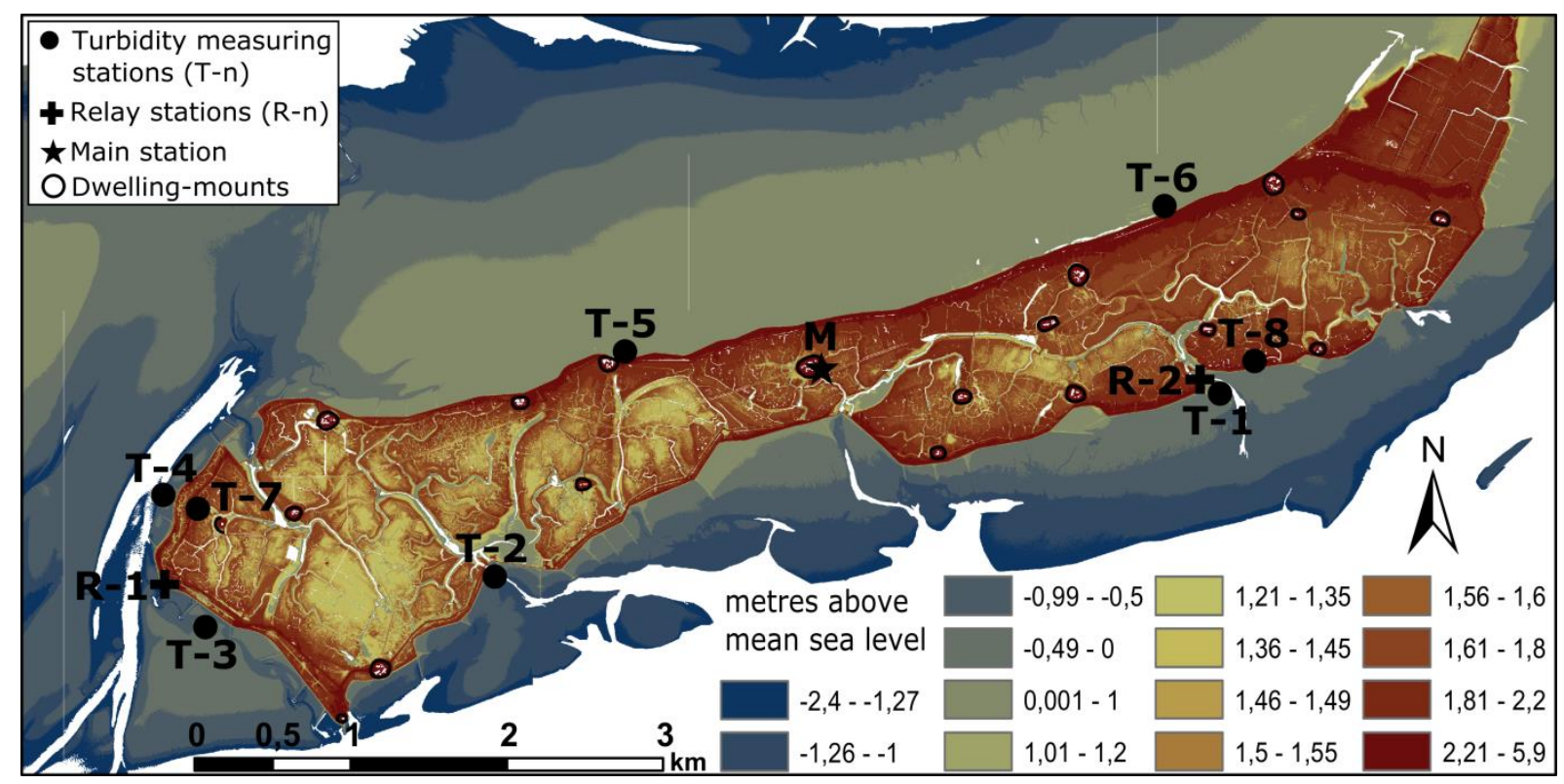

Figure 2-2: Digital ground model (based on Laser scan DGM1 data from 2014 courtesy of LKN.SH) of Langeness with the locations of the different components of the autonomous network of turbidity measuring stations and transmission units. The white areas show depths that cannot be detected by laser scanning due to high water coverage. The three white vertical lines are artifacts. 
Table 2-1: Topographical survey of all turbidity measurement stations in meters above MSL. The exact locations of the four measuring levels from top to ground are illustrated in Figure 2-4.

\begin{tabular}{lcccccccc}
\hline station & T-1 & T-2 & T-3 & T-4 & T-5 & T-6 & T-7 & T-8 \\
\hline top & 2.62 & 1.40 & 1.97 & 1.39 & 3.31 & 3.33 & 4.31 & 4.60 \\
OBS & 0.71 & -0.50 & 0.13 & -0.50 & 1.37 & 1.45 & 2.38 & 2.66 \\
grid & 0.51 & -0.70 & -0.07 & -0.70 & 1.17 & 1.25 & 2.18 & 2.46 \\
ground & 0.14 & -1.07 & -0.50 & -1.08 & 0.83 & 0.86 & 1.83 & 2.13 \\
\hline
\end{tabular}

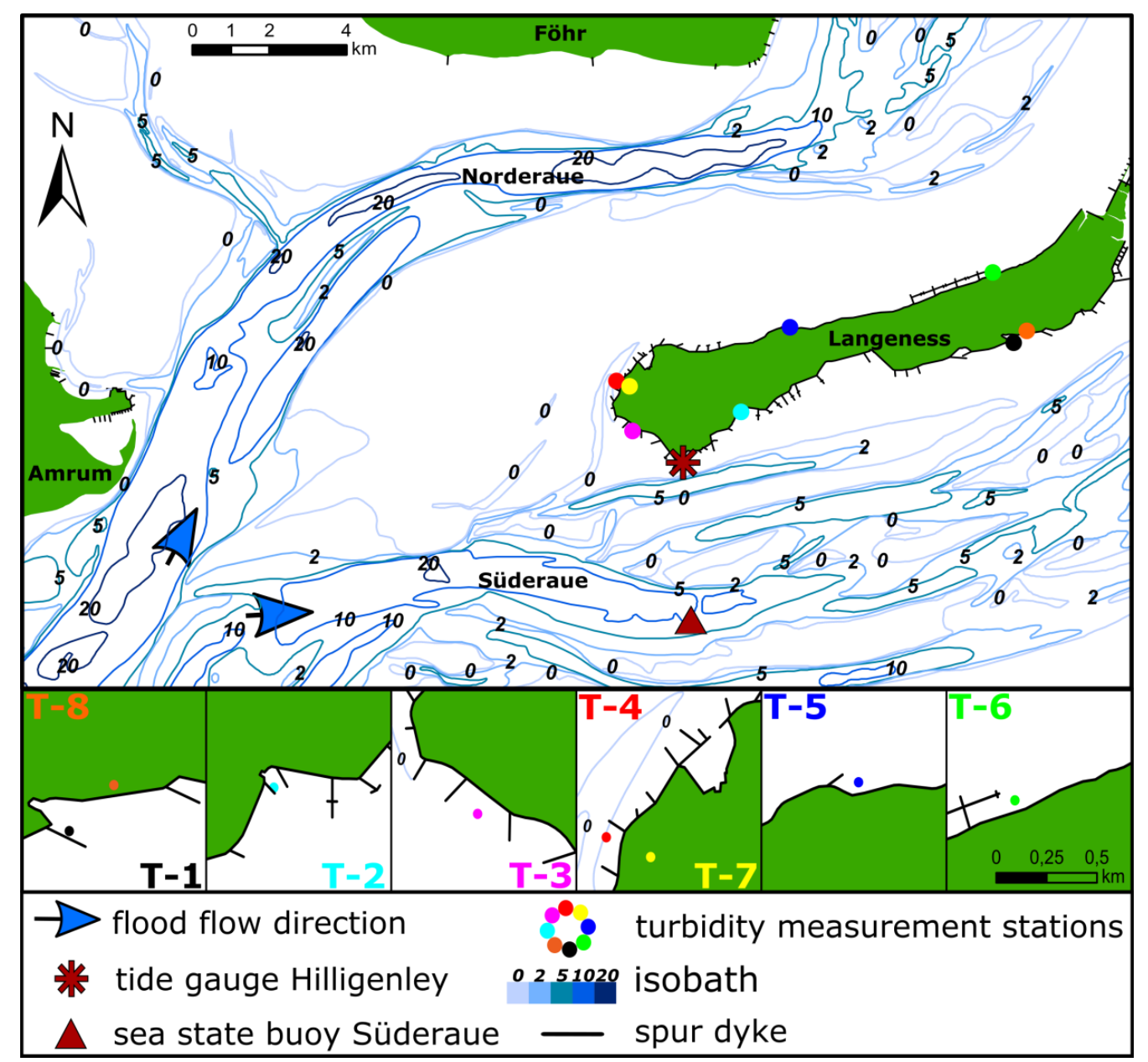

Figure 2-3: Bathymetric map (courtesy of Bundesamt für Seeschifffahrt und Hydrographie) showing the two large tidal channels Norderaue in the north and Süderaue in the south enclosing Langeness. The small pictures show a detailed view of the respective turbidity measurement stations with their neighboring spur dykes in the tidal flats except for station T-6 which is located next to a land reclamation area.

The tide is semidiurnal with an average mean tidal range of $2.74 \mathrm{~m}$ measured at tide gauge Hilligenley during the winter measuring period from October 1, 2017 to February 15, 2018. Such range is characteristic for mesotidal regimes according to Hayes (1979). In a typical incipient high tide, the tidal channels radially widen in the flood flow direction and enclose 
Langeness fan-like. In case of an inundation event the water overflows the dykes and inundates the entire Hallig, solely the dwelling-mounts stand above the water. The different locations of the individual stations are also affected by coastal protection measures. In order to keep the tidal channels at some distance from the edge of the Hallig, spur dykes have been installed to protect against flow induced erosion. Almost all stations are located in the vicinity of the outer end of spur dykes except station T-6, which is located adjacent to a land reclamation area.

Beyond the measuring stations, the tidal flat sediments around Langeness were investigated in a survey covering every $100 \mathrm{~m}$ within $500 \mathrm{~m}$ long transects perpendicular to the shore line. The results of 1280 samples analyzed by finger tests, 113 of them verified by laser particle sizer, using the classification of Folk and Ward (1957) revealed a mixed sediment flat with predominantly fine sand along with a small increase of the grain size towards the tidal channels. Only the area around station T-2 revealed a tidal flat with a finer grain size distribution with a higher proportion of silt-sized material. The grain-size distribution of sediments from the tidal channel Norderaue is dominated by medium sand. In the tidal channel Süderaue, medium sand only occurs in the deeper levels whereas the shallower levels are dominated by fine sand (Asmus et al. 2016).

\subsubsection{Turbidity data acquisition}

We installed eight turbidity measurement stations (Figure 2-2). Six of them are distributed on the tidal flat around Langeness (T-1 to T-6) and two of them are installed on Langeness itself ( $T$ 7, T-8). Table 2-1 shows the topographical survey of all stations. Figure 2-4 shows a detailed illustration of individual components of a turbidity measuring station. 


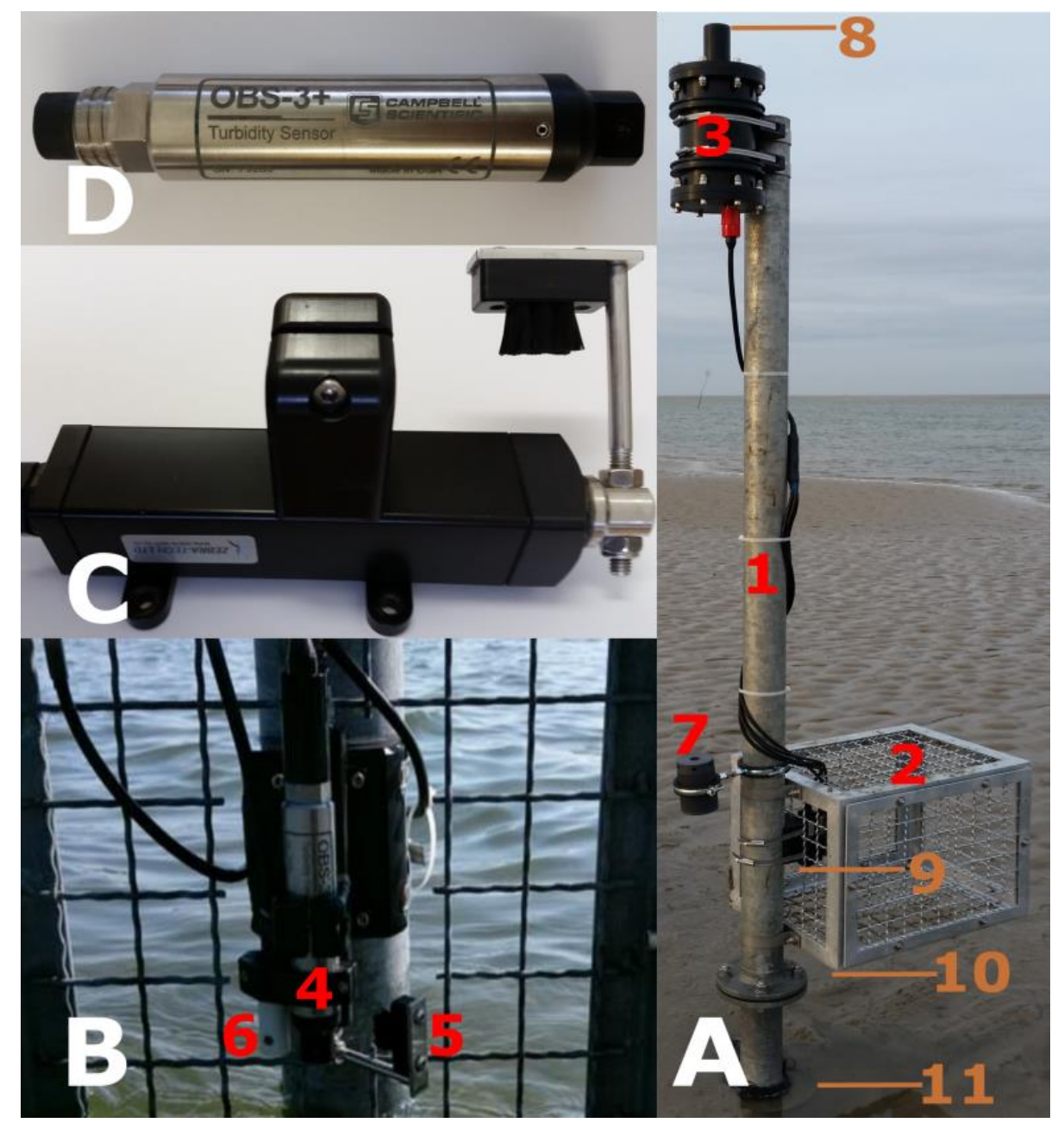

Figure 2-4: Illustration of the turbidity measuring stations: $(A)$ construction of a turbidity measuring station (T-4) located on the tidal flat; (B) entire sensor unit inside the metal grid cage; (C) wiper; (D) OBS3+ sensor. The components: 1 metal post, 2 metal grid cage, 3 POM pressure housing, 4 OBS sensor, 5 wiper, 6 probe, 7 sediment accumulator installed in the field. The topographical survey points of Table 2-1: 8 top, 9 OBS measuring height, 10 grid and 11 ground.

All components are attached to a $2.3 \mathrm{~m}$ high metal post. At the upper end of the metal post there is a polyoxymethylene (POM) pressure housing, in which the data acquisition (CR200X datalogger by campbell@ scientific) and transmission unit (RF422 radio by campbell@ scientific) is located. The power supply of the measurement station is ensured by two battery packs with four 3.6 $\mathrm{V}$ lithium batteries each connected in series. The heart of the measuring station is an optical backscatter turbidity sensor (OBS-3+ by campbell (C scientific) in a titanium case to protect it from seawater. This OBS operates in the near-infrared wavelength range of $850 \pm 5$ $\mathrm{nm}$ and receives the backscatter signal at a scattering angle between 140 and 160 degrees to minimize interferences of air bubbles and organic matter. The sensor is mounted about $0.5 \mathrm{~m}$ above ground with the OBS photodiode pointing to the north to avoid saturation effects by sunlight. A more detailed description of the OBS-3+ is given in Campbell Scientific (2017). A 
probe is located at the same level next to the OBS, which detects the water level simply by conductivity. Behind the OBS, a wiper (Hydro-Wiper by Zebra-Tech Ltd) is attached, which cleans the OBS sensor and the probe before each measurement cycle. The measurement cycle starts as soon as the probe is permanently immersed in the water for 10 minutes and stops as soon as the water level drops below the probe. This prevents erroneous measurements that would be induced by wave impact. The entire sensor unit is enclosed with a $0.4 \times 0.5 \times 0.4 \mathrm{~m}$ metal grid cage to protect it against, e.g., flotsam.

\subsubsection{Data transmission (networking)}

Each turbidity measurement station is equipped with a Low-Profile-Antenna which transfers the data within the license-free ISM bands in the $869 \mathrm{MHz}$ range to the main stations radio (RF407 by campbell (C scientific) which in turn feeds the data into the mobile network and finally sends it to the receiving institute server at University of Göttingen. Regarding the weak transmission power and the challenges of an undisturbed line of sight behind the protection constructions and the variety of dwelling-mounts on the Hallig, two relay stations were installed to achieve an undisturbed fresnel zone between the transmitter and the receiver and thereby avoid the excessive attenuation of the signal (Figure 2-2). The data are transmitted immediately after the measurement has stopped. If transmission is not possible, the radio within the turbidity stations automatically turns off after one hour to save battery power. The next transmission attempt restarts after the subsequent measurement. In case of a larger network problem, turbidity data can be stored for up to one month. With this setting, all turbidity measurement stations are networked and measure autonomously since February 25, 2017.

\subsubsection{Time series}

Between February 25, 2017 and February 15, 2018 our turbidity network has achieved measurement durations between 45 and 215 days at single measurement stations with a measuring interval of $1 \mathrm{~min}$ (Table 2-2). The total measurement duration depends on station location (mainly altitude) and flooding frequency. Lower stations therefore have much longer measurement duration than stations in higher areas due to their longer flooding durations. Four inundation events have been recorded in that time frame. The working time and number of recorded inundations are shown in Table 2-2 for each station. The inundation events occurred on October 29, 2017, December 8, 2017, January 4, 2018 and February 11, 2018. Despite some issues with maintenance or technical failures, three of the four storm surges 
could be measured simultaneously at all stations in the tidal flat. The four recorded storm surges were moderate regarding tidal elevation and therefore no measurements could be triggered simultaneously from the two stations in the marshland (T-7 and T-8) so far. The calibrated turbidity data is presented together with water level, wind speed and direction and significant wave heights. The water level data is collected at the tide gauge Hilligenley located in the southwest of Langeness and the wave data is collected at a sea state buoy located in the tidal channel Süderaue (Figure 2-3). The wind data is collected at the weather station Strucklahnungshörn located at $54.4965^{\circ} \mathrm{N}$ and $8.8069 \mathrm{E}$, about $22 \mathrm{~km}$ southeast of the tide gauge Hilligenley.

Table 2-2: Total measurement duration in days from February 25, 2017 to February 15, 2018 and the number of recorded storm surge events within the winter measurement period from October 1, 2017 to February 15, 2018.

\begin{tabular}{ccc}
\hline station & $\begin{array}{c}\text { measuring } \\
\text { duration [days] }\end{array}$ & $\begin{array}{c}\text { number of } \\
\text { recorded storm } \\
\text { surges }\end{array}$ \\
\hline T-1 & 160 & 4 \\
T-2 & 215 & 4 \\
T-3 & 106 & 3 \\
T-4 & 183 & 3 \\
T-5 & 57 & 4 \\
T-6 & 45 & 4 \\
\hline
\end{tabular}

\subsubsection{OBS Calibration}

In order to sample SPM resembling the one detected at each OBS, we collected sediment samples at each station with a sediment accumulator (Design by IM+P engineering office Dr.Ing. Manzenrieder und Partner GbR http://www.imp-ol.de) (Figure 2-5) mounted next to the OBS measuring position (Base). A second accumulator (Top) was mounted approx. $1.2 \mathrm{~m}$ higher to test for the homogeneity of SPM in the water column. The sediment accumulators have a capacity of approx. $350 \mathrm{~cm}^{3}$. Its six inlets of $8 \mathrm{~mm}$ in diameter and the closed top minimize resuspension of the sample inside the accumulator at higher flow velocities. Furthermore, with an aspect ratio of 3.6 the accumulator fulfills the recommended aspect ratio conditions by Gardner (1980a, 1980b) to prevent resuspension. The sediment accumulators are emptied after 
each storm surge event and, if possible, directly before. The accumulators are capable to collect sediments for about one month depending on the location before they get plugged and have to be emptied. So far samples of four storm surges and two one month periods under fair weather conditions were collected. These samples were analyzed for particle size and organic content. Sediments collected during the fair weather period January 8, 2018 to February 9, 2018 were taken for the calibration of the individual OBS sensors. We preferred this method because bed sediment from the flats directly below the stations (which might be used alternatively) shows a much coarser grain size compared to the material in the accumulators (Table 2-3). This difference in grain sizes is consistent with results by Poerbandono and Mayerle (2005) who found up to 5 times bigger mean grain sizes in bed sediments compared to suspended material collected at fair weather conditions.

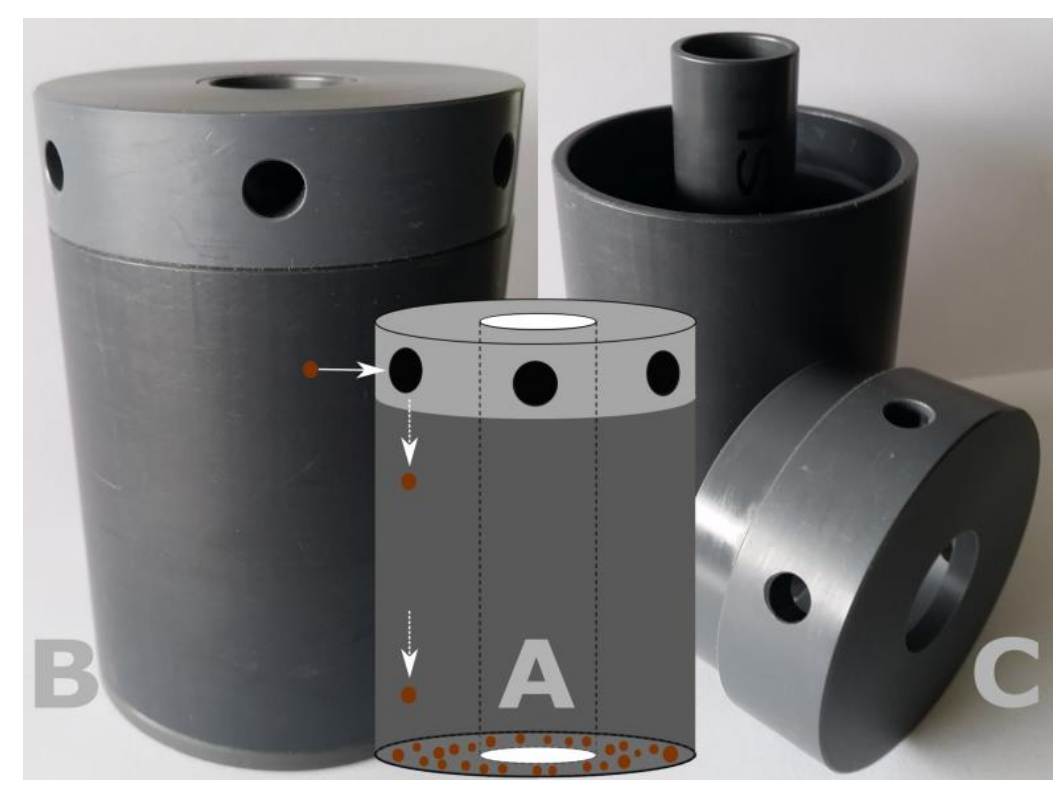

Figure 2-5: (A) Sketch of a sediment accumulator. Once a particle enters one of the six inlets of the accumulator and is no longer affected by the outer flow conditions, it should sink to the accumulator bottom. (B) Close up of sediment accumulator closed and opened (C). 
Table 2-3: d50 percentile (median) of bed sediment collected next to each measurement station and Base accumulator sediment samples which we have used for OBS calibration collected at each station over a period of one month from January 8 to February 9, 2018 without occurring storm surge events. The measurements were carried out with a laser particle sizer LS13320 by Beckman\&Coulter.

\begin{tabular}{ccc}
\hline station & $\begin{array}{r}\text { bed sediment } \\
\mathrm{d} 50[\mu \mathrm{m}]\end{array}$ & $\begin{array}{c}\text { accumulator } \\
\text { sample } \\
\mathrm{d} 50[\mu \mathrm{m}]\end{array}$ \\
\hline T-1 & 143 & 46 \\
T-2 & 40 & 13 \\
T-3 & 162 & 31 \\
T-4 & 153 & 107 \\
T-5 & 146 & 18 \\
T-6 & 114 & 19 \\
\hline
\end{tabular}

All individual calibration samples were prepared in the same way. The samples were wet sieved to filter out seagrass and shells. Subsequently, the sieved sediment samples were stirred with an electric paint stirrer and sufficient water within a bucket to get a homogeneous suspension. While stirring, a $100 \mathrm{ml}$ aliquot was removed from the suspension in order to determine the SPM content by oven drying $\left(105^{\circ} \mathrm{C}\right)$ and weighting. A correction for dissolved salt was applied via conductivity measurement. Knowing the SPM content allows for taking suitable SPM aliquots from the suspension for the calibration procedure. For error assessment regarding an individual aliquot from the suspension we took five $100 \mathrm{ml}$ aliquots from the suspension and recorded the standard deviation of the evaluated mean value.

For the calibration procedure a special calibration tank was built (Figure 2-6). Its operation mode is mainly based on efficient circulation of the contained suspension to ensure homogenous distribution. The tank consists of a $1.65 \mathrm{~m}$ long black polyethylene (PE) tube with a diameter of $0.2 \mathrm{~m}$ which is heat sealed with a PE base plate on the ground. At the bottom of the container a pump is circulating up to $40 \mathrm{I}$ suspension within a $0.04 \mathrm{~m}$ diameter pipe system into the upper area of the container that is described as turbulence chamber. The penetration depth of the pipe is deep enough to ensure a closed circulation of the suspension and to avoid the entrainment of air bubbles during the calibration procedure. The turbulence chamber is separated from the calibration chamber by a sieve. This separation has been installed to minimize "hydrodynamic noise" (Black and Rosenberg 1994), i.e. erroneous measurements 
triggered by turbulent flow conditions (e.g. Benns and Pilgrim 1994) and to reach the best possible homogeneous suspension in the calibration chamber in which the OBS is mounted (Figure 2-6).

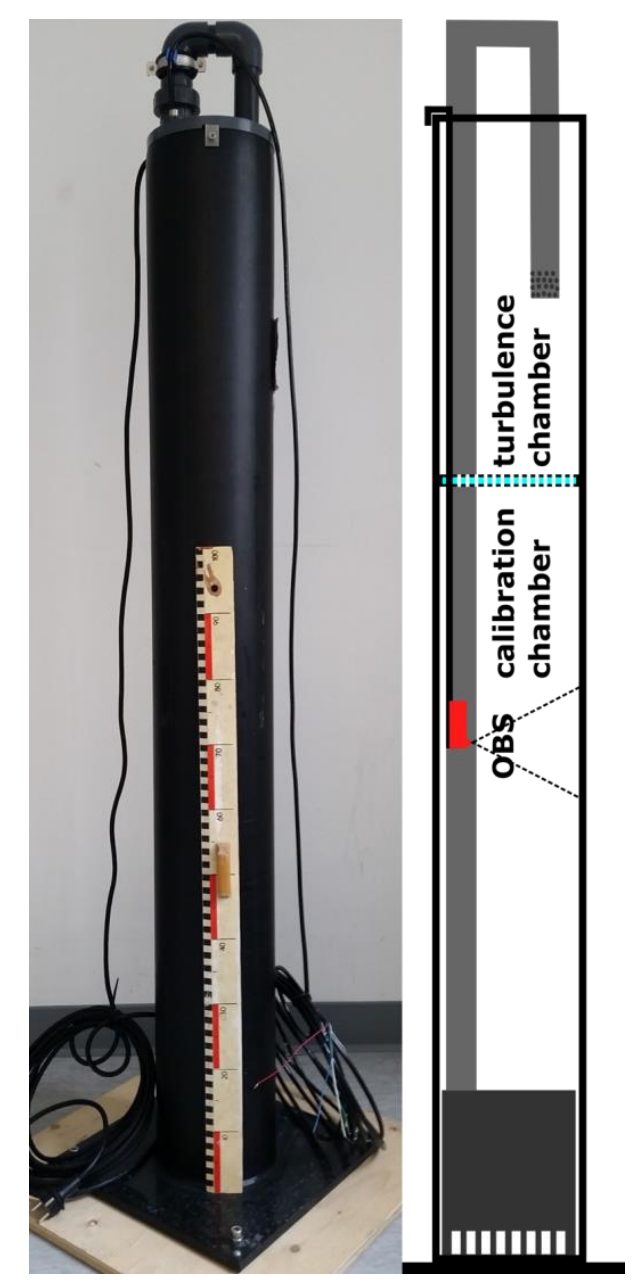

Figure 2-6: Figure and sketch of the calibration tank. The tank consists of a $1.65 \mathrm{~m}$ long and $0.2 \mathrm{~m}$ wide black PE tube. The sieve (cyan) inside is used for separating the turbulence and the calibration chamber. The pump on the ground is coloured in dark gray. It pumps the suspension within a $0.04 \mathrm{~m}$ wide pipe system (light gray) into the upper turbulence chamber of the tank. The OBS (red) is mounted in the calibration chamber.

Each OBS sensor was calibrated with the respective accumulator calibration sample. Before each calibration procedure, the signal strength in deionised water was measured in order to determine the offset. The offset depends on the used OBS ranging from 8 to 13 millivolts and was subtracted in subsequent measurements. The suspension was circulated during the whole calibration process. Step by step $7-13$ aliquots of the previously described calibration suspension were added. After each added aliquot the OBS response was recorded for 60 seconds and averaged. This average was assigned to the current SPM content in the calibration tank. 
To verify the accuracy of the calibrated data, 55 in situ water samples were collected in the vicinity of the stations with a 1 I water sampler. These samples were vacuum filtered $(0.45 \mu \mathrm{m})$. The sea salt was rinsed out with deionised water during the filtration process. The SPM content was measured by oven drying $\left(105^{\circ} \mathrm{C}\right)$ and weighting.

Additionally, for each sensor a separate reference measurement was performed with an artificial sample of known SPM content in order to obtain additional information on the accuracy of the calibration run.

\section{$2.3 \quad$ Results}

\subsubsection{Laboratory calibration}

The calibration data is shown in (Figure 2-7). The OBS response is strongly influenced by the PSD of the used accumulator sediment. The finer the PSD of the calibration sample, the higher is the OBS response implying that the slopes of the linear regression lines decrease with decreasing PSD (see medians in Table 2-3). The single reference measurements generally show good consistency with the calibration curves. Higher accuracy was achieved for low SPM concentrations, which is probably due to better homogenisation of the suspension during the measurement in the PE bucket (see Figure 2-7, T2+T4). 


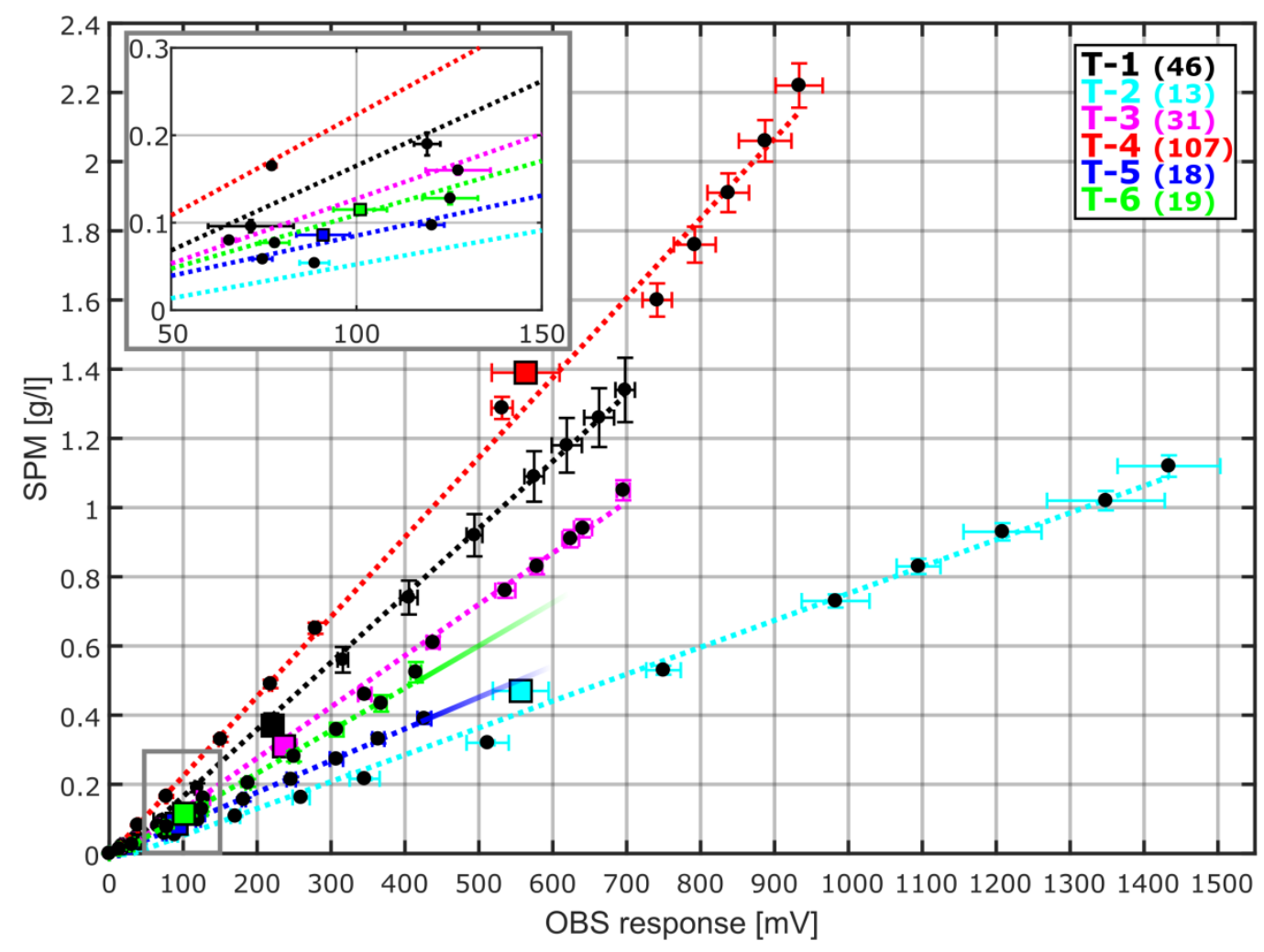

Figure 2-7: Calibration results of stations T-1 to T-6 using the accumulator calibration samples (respective median grain size in $[\mu \mathrm{m}]$ in brackets) and the individual incrementally added aliquots (series of black dots for each station). The corresponding linear polynomial fits are shown with dotted lines. The slopes of the regression lines continuously increase with increasing median grain size. The blurred straight lines at the end of the polynomial fit for stations T-5 from $0.38 \mathrm{~g} / \mathrm{l}$ and T- 6 from $0.5 \mathrm{~g} / \mathrm{l}$ indicate the extrapolation range towards higher SPM concentrations. The extrapolation of the calibration curves for these two stations was necessary to cover all measured concentrations from the field, because not enough accumulator sediment was available to extend the range by a further calibration point. The vertical error bars estimates possible deviations of the added aliquot as determined by mean and standard deviation of five aliquots taken from the respective suspension. The horizontal error bars show the standard deviation of the measured OBS signal. The coloured squares show the results of the reference measurements. The inset gives a more detailed view for low OBS response (between 50 and $150 \mathrm{mV}$, grey box).

The in situ samples (see 2.2.5) taken in the field show only little scatter with respect to the calibration curves, as illustrated in Figure 2-8. Out of ten samples taken at station T-6, seven samples plot within the $95 \%$ confidence interval of the linear regression line and two samples plot within the $99 \%$ confidence interval. One sample is an outlier with SPM concentration of $0.24 \mathrm{~g} / \mathrm{l}$. An even better result was achieved at station T-1 where nine out of ten in situ samples plot within the $95 \%$ confidence interval. The largest deviation between the regression line and the in situ samples was observed at station T-4 with results in the $99 \%$ confidence interval only. This is most likely due to difficult conditions during field sampling within a tidal channel at 
higher flow velocities. Regression analysis of the in situ samples is not possible because samples with higher SPM concentrations would require boat operation under harsh conditions, which could not yet be implemented here, mainly for reasons of safety.

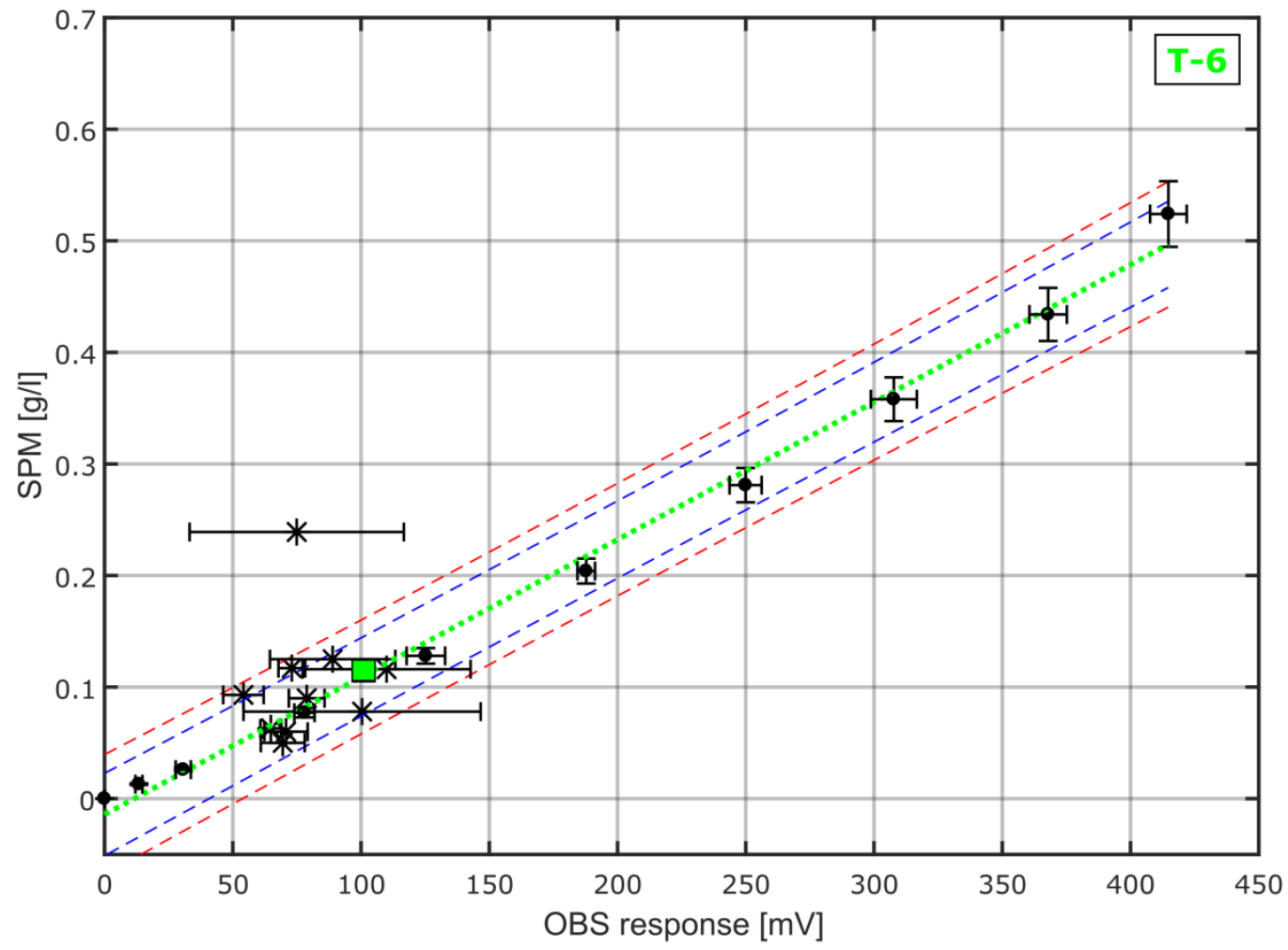

Figure 2-8: Calibration result of station T-6 with accumulator sediment calibration samples and their individual incrementally added aliquots (series of black dots). Error bars as in Figure 2-7. The corresponding linear polynomial fit is shown with a dotted green line, the $95 \%$ confidence intervals with blue dashed lines and the $99 \%$ confidence intervals with red dashed lines. The green square shows the result of the single reference measurement. The stars show the results of ten in situ samples. One in situ sample is an outlier with $0.24 \mathrm{~g} / \mathrm{I} \mathrm{SPM}$.

\subsubsection{Field measurements}

\subsubsection{Single tidal cycles}

The SPM concentrations around Hallig Langeness vary spatially and temporarily. The comparison of two tidal cycles at fair weather and storm conditions indicates striking differences in the determined SPM concentrations in the tidal flats around Langeness (Figure 2-9). 

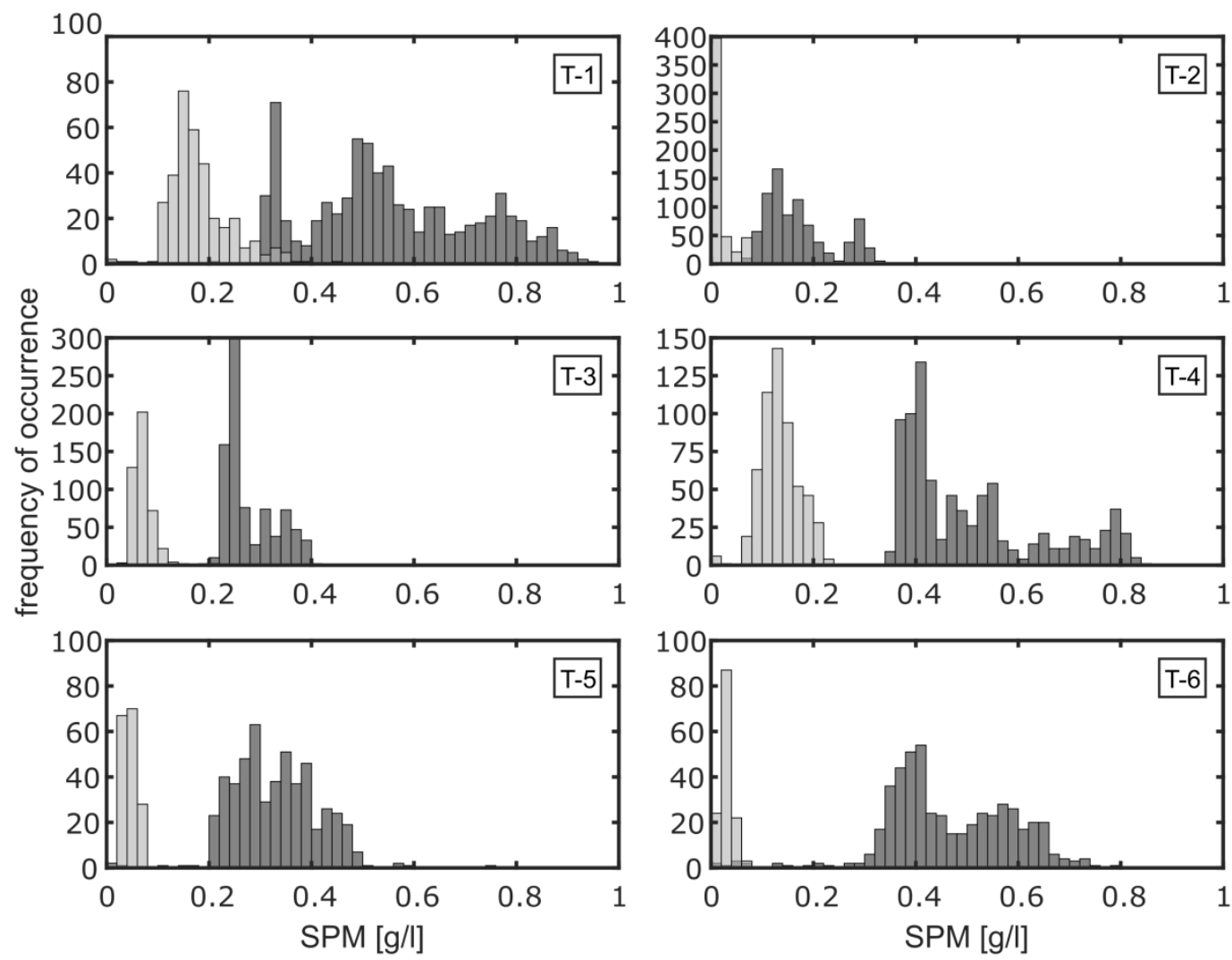

Figure 2-9: Frequency distributions of SPM concentrations during two tidal cycles under different weather conditions. The light grey bars show a common tidal cycle under fair weather conditions recorded on November 30, 2017. The dark grey bars show the SPM concentrations during an inundation event within a storm surge in the night of February 11 to 12, 2018.

The tidal cycle at fair weather conditions was recorded on November 30, 2017 and the one at storm surge conditions during an inundation event on February 12, 2018. Under fair weather conditions, particles are suspended only by tidal induced flow conditions. Under fair weather conditions, the SPM concentration is usually highest at station T-4 due to the proximity to a branch of the tidal channel Süderaue and at station T-1 due to an unhindered access to the tidal channel Süderaue (Figure 2-3) leading to the highest SPM concentrations $>0.2 \mathrm{~g} / \mathrm{l}$. Although station T-2 is located closer to the tidal channel Süderaue, it shows the lowest SPM values and the least variability due to its shielded position between two spur dykes. With increasing distance to the tidal channels, the tidal currents are reduced, which in turn leads to a reduction of the SPM concentrations. This is visible, for instance, at station T-3 showing roughly half the SPM concentrations of station T-4. Rather low SPM concentrations $<0.1 \mathrm{~g} / \mathrm{l}$ were measured at the stations in the north (T-5 and T-6). They have the highest elevations and are located over 1 $\mathrm{km}$ distance from the tidal channel Norderaue (Figure 2-2, Table 2-1), resulting in relatively low tidal currents along the northern shore of Hallig Langeness. 
During storm surge conditions, the hydrodynamic processes are supposed to change considerably with increasing tidal currents, higher coastal set-up and intensified wave activity. Wind speed and significant wave height recorded at the sea state buoy Süderaue during the period from October 1, 2017 to February 15, 2018 show a significant positive correlation with a coefficient of determinations of 0.68 within 19719 numbers of observations. These wave heights cannot be extrapolated to the whole area around Langeness, because they are only measured stationary at a single sea state buoy in the tidal channel Süderaue. However, due to the strong correlation between wind speed and wave height they can be used for the qualitative, large-scale assessment of the sea state conditions in our study area. Thus, under harsh weather conditions and top wind speeds between 16.7 and $27.8 \mathrm{~m} / \mathrm{s}$, wave height will increase and hence the resuspension of bed sediments should increase, too. This will occur especially in shallow water areas, e.g. at stations T-1, T-5 and T-6 (Table 2-1).

During the storm surge cycle in February 2018, all stations show higher SPM concentrations than during the fair weather cycle (Figure 2-9). The different ranges in SPM concentrations of the individual stations indicate to an underlying variability in the local hydrodynamic conditions. Stations T-1 and T-4 showed the highest SPM concentrations at fair weather conditions and also at storm surge conditions. The smallest SPM concentrations occurred at station T-2, which already showed the lowest SPM concentrations at fair weather conditions. The other three stations (T-3, T-5, T-6) are intermediate with respect to SPM concentrations, but all show a significant increase from fair weather to storm surge conditions (Figure 2-9). In the north of Langeness (T-5, T-6), the higher elevation of the tidal flat and lower water depths likely lead to increased resuspension and prevent settling of particles due to wave activity. T-3 and T-4 seem to be dominated by tidal currents and the area of station T-1 seems to be influenced by both, tidal currents and wave activity. The increase at T-2 remains small despite harsh weather conditions. The level of SPM concentrations under fair weather conditions and harsh weather conditions shows that the SPM concentration is usually highest at stations with an unobstructed access to a tidal channel or a position unsheltered to wind and waves. Note that for harsh weather conditions the SPM frequency distributions change from unimodal to polymodal in most cases. What conditions and processes lead to this change in modality will be discussed in the following.

Figure 2-10 illustrates the relations of SPM, wind speed, wind direction, significant wave height and tidal elevation as measured for the storm surge in February, 2018. Four consecutive tidal 
cycles were selected. The first tidal cycle (Feb. 11) is accompanied by increasing wind speed with gradual change in wind direction from SE to SW. With the increase in wind speed, the wave height also increased. At the beginning the southeasterly wind direction remained stable for several hours and led to the highest measured SPM concentration at station T-2. So far, the tidal elevation was not high enough to trigger a measurement at stations T-5 and T-6. The second tidal cycle reached a much higher elevation than the first leading to inundation of the Hallig. Tidal elevation was high enough to trigger a measurement cycle at all tidal flat stations around Langeness. Within this tide the averaged wind speed reached its maximum along with the highest waves and overall highest SPM concentrations. Only station T-2 showed a steadily decreasing SPM concentration from the early first cycle. During the third tidal cycle, wind speed and wave height started to decrease accompanied by overall decreasing SPM concentrations. The water level dropped rapidly at the ebb stage of the third tidal cycle and all stations stopped measurements in order of their respective elevation. The fourth tidal cycle led to a renewed flooding of all stations in the tidal flats starting a new measuring cycle. The measured SPM values, however, decreased again according to the smoother weather condition. 


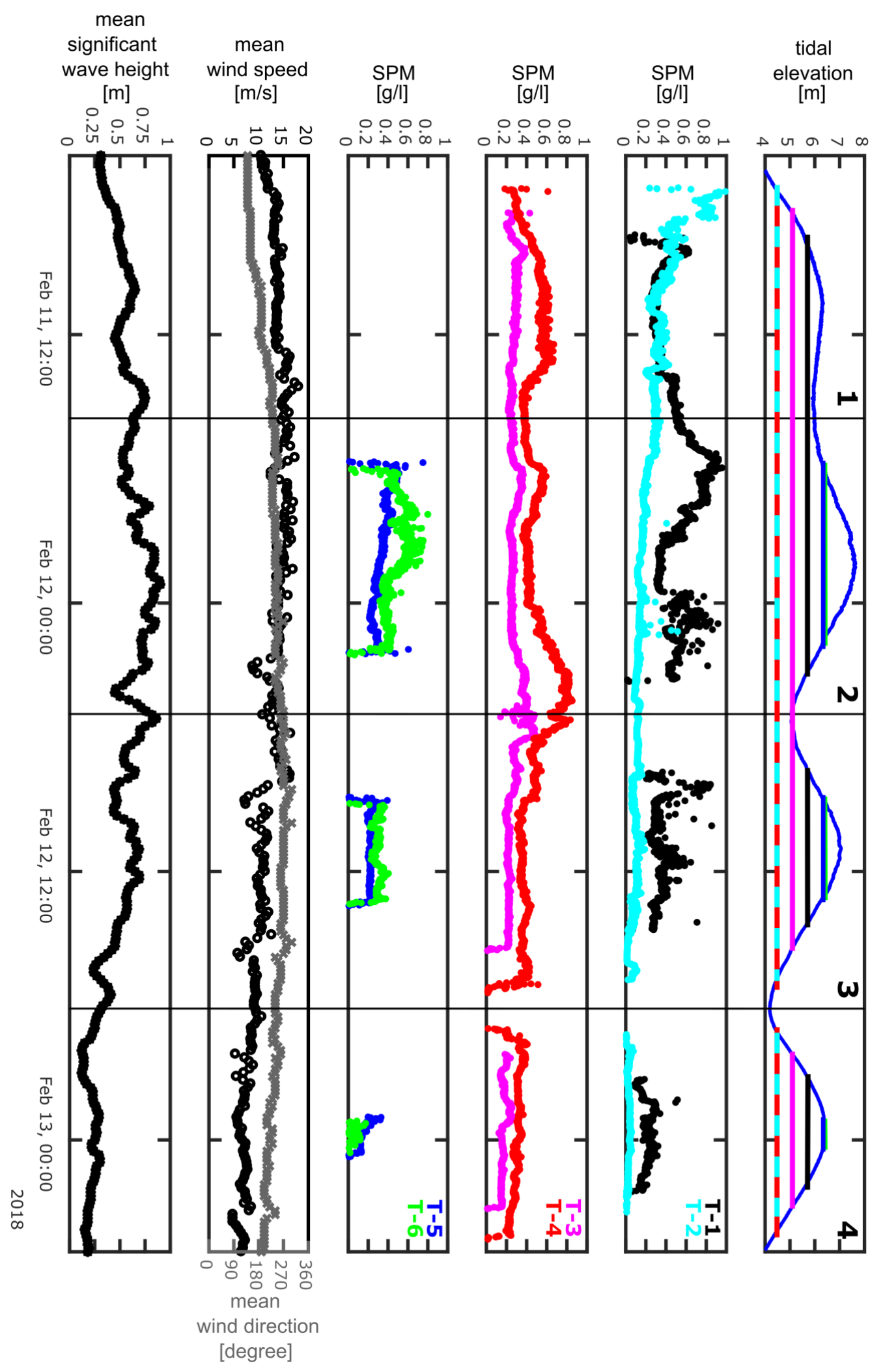

Figure 2-10: A section of our measured time series shows SPM concentrations for four consecutive tidal cycles for an inundation event in February 2018. Right column shows the measuring duration of the individual stations (vertical coloured bars) in relation to the tidal elevation (tide gauge Hilligenley): T1=black, $\mathrm{T}-2=$ =cyan, $\mathrm{T}-3=$ magenta, $\mathrm{T}-4=\mathrm{red}, \mathrm{T}-5=\mathrm{blue}, \mathrm{T}-6=$ green. Additional information include, tidal elevation, ten minutes averaged values of wind speeds (circles) and wind directions (crosses) recorded at the weather station Strucklahnungshörn and ten minutes averaged values of significant wave heights from the sea state buoy Süderaue. 
During this time series, fluctuations in the SPM concentration within the individual tidal cycles are recognized, which calmed down in their intensity only in the fourth tidal cycle. In the slack water phase during the first high tide, a drop in the measured SPM is recorded at station T-1 (Figure 2-10). Further drops in SPM concentrations are observed in the slack water phases during low tide between the first and second tidal cycle at stations T-3 and T-4, and around high tide of the second tidal cycle at stations T-1, T-3, T-4 and T-6. Slack water conditions are prime candidates for sediment settling and, consequently, the SPM concentrations decrease. This process seems to be subdued when low water levels coincide with wind induced waves on the tidal flats causing resuspension of sediment and thus a remarkable increase of the SPM concentration. This effect can be assumed during slack water at low tide (i) after the first tidal cycle at station T-1 and (ii) after the second tidal cycle at stations T-3 and T-4. Further increases in SPM concentration were recognized at the inflexion points of the tidal cycle (mid-tide) where tidal currents are highest (e.g. T-1, T-3, T-4 within the second tidal cycle). The SPM response to increasing tidal currents may show a slight offset in time at stations located farther away from the recording tide gauge (i.e. in the North of Langeness). Such time-delayed response is recorded in the second tidal cycle at station T-6.

This complex web of interacting hydrodynamic processes related to changing flow conditions and wave impact within single tidal cycles likely induces the change in modality between fair weather and storm conditions as we have clearly seen in Figure 2-9.

\subsubsection{Wind conditions}

The SPM concentration depends on the meteorological and associated hydrodynamic conditions. The latter vary considerably from location to location within the study area as outlined before. However, to achieve an increased SPM at a specific location within the tidal flats a suitable wind direction is crucial (e.g. T-2, first tidal cycle, Figure 2-10). When looking at a longer period, this becomes more obvious. Figure 2-11 shows ten-minutes averages of wind speed and direction during two longer measurement periods from October 1, 2017 to February 15, 2018 (winter period) and from February 25, 2017 to June 1, 2017 (spring period). The period between June and September 2017 is not considered, as intense algae and barnacle growth on the stations compromised the measurements during this time.

During the winter period the highest averaged wind speeds with up to $27,8 \mathrm{~m} / \mathrm{s}$ were recorded during inundation event 1 (Table 2-4). In a density plot of wind speed and direction the 
conditions during inundation event 1 are clearly outstanding (see Figure 2-11, box 1). The most common directions for wind speeds around $10 \mathrm{~m} / \mathrm{s}$ are southwest to west in both winter and spring season, while lower wind speeds around $5 \mathrm{~m} / \mathrm{s}$ are most frequently related to southeastern directions. Overall, the spring period is characterized by lower maximum wind speeds compared to the winter period. Only easterly winds show higher maximum speeds compared to the winter period (Figure $2-11$ box 2$)$. Wind directions from SSE $\left(140-170^{\circ}\right.$, Figure 2-11, box 3) and northerly winds show remarkably lower wind speeds compared to the winter period.

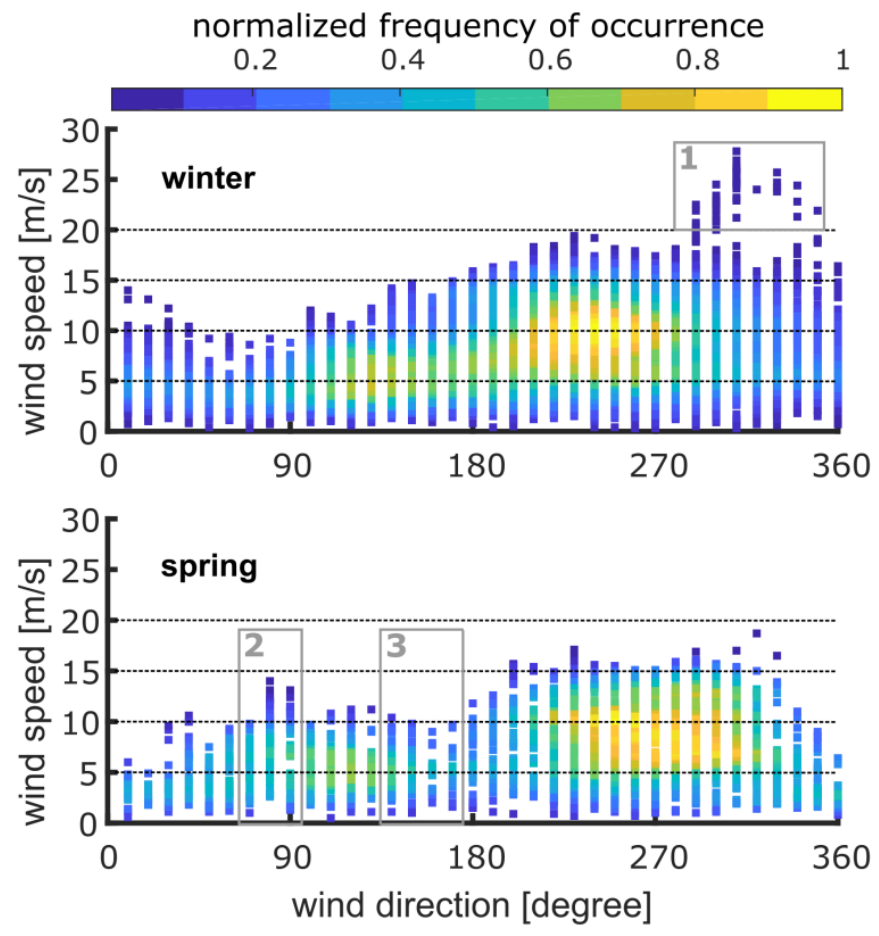

Figure 2-11: Ten minutes averaged wind speed and direction during the measuring period from October 1, 2017 to February 15, 2018 (winter) and from February 25, 2017 to June 1, 2017 (spring) recorded at the weather station Strucklahnungshörn. The grey box 1 contains the highest wind speeds occurring during the first storm surge event with wind speeds above $20 \mathrm{~m} / \mathrm{s}$ (up to $28 \mathrm{~m} / \mathrm{s}$ ). The grey box 2 highlight higher maximum wind speeds compared to the winter period. Wind speeds from about northern and SSE directions (box 3 ) are lower compared to the winter months. The colour bar visualizes data density.

\subsubsection{Winter time series}

The patterns of SPM concentration at individual stations are related to the measured wind direction and speed. All stations show increased SPM concentrations around the most frequent wind directions (Figure 2-12). However, some of the patterns differ significantly from each other due to the conditions at the individual locations. 


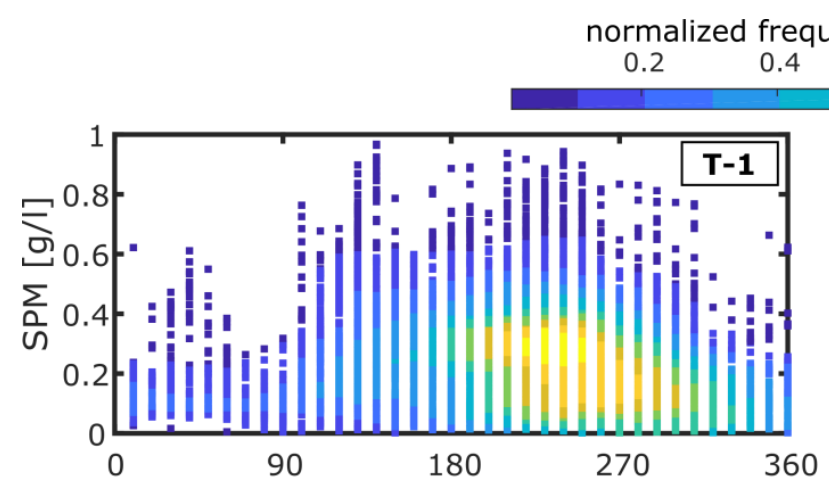

$\begin{array}{ll}0.6 & 0.8\end{array}$
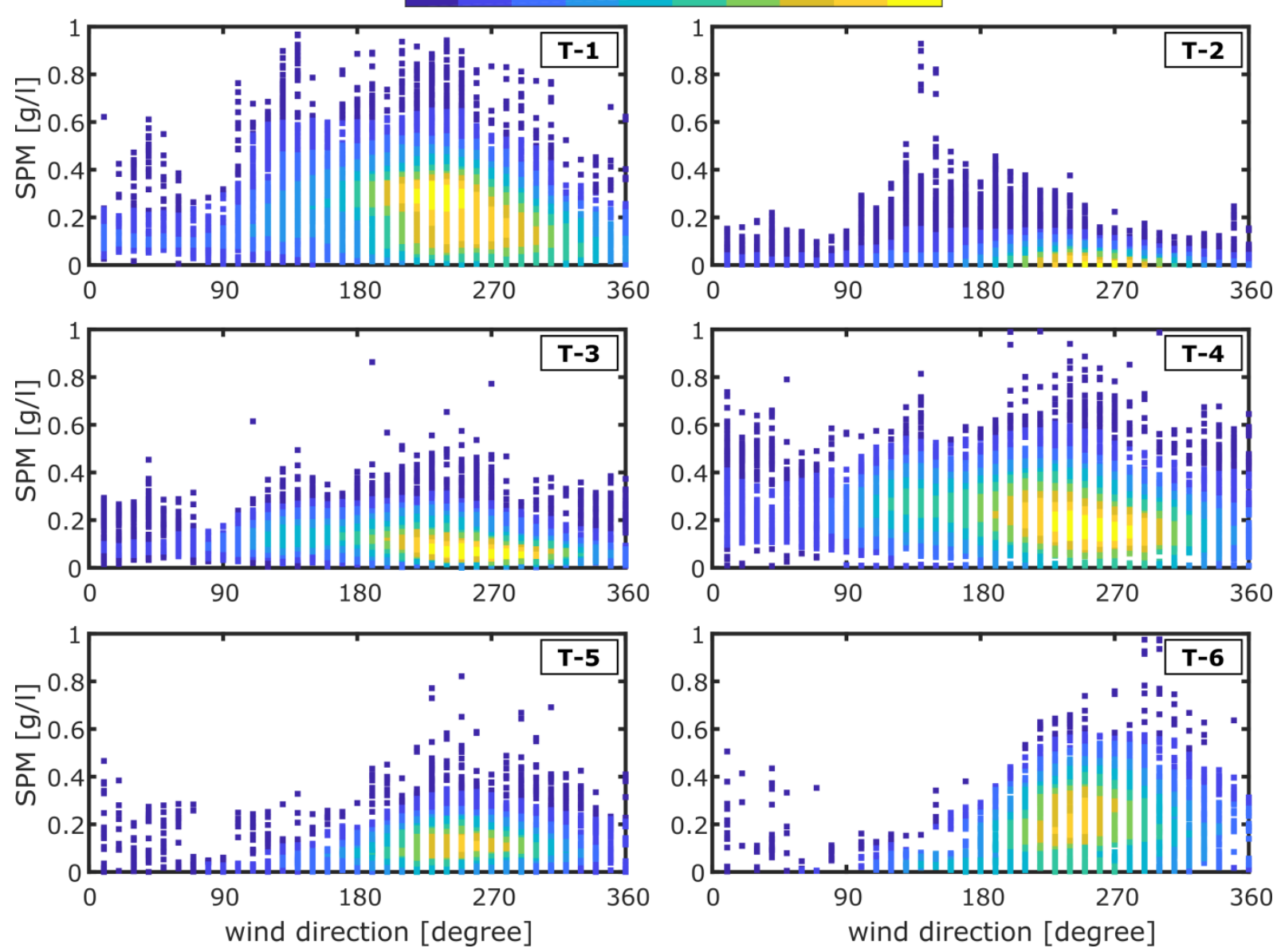

Figure 2-12: Density plot of SPM versus wind direction of all stations in the tidal flats measured during the same winter period from October 1, 2017 to February 15, 2018 as in Figure 2-11. The colour bar visualizes data density.

The stations in the south of Langeness (T-1 and T-2), for instance, have nearly the same pattern but station T-2 is strongly attenuated, which reflects the specific sheltered position of this station as outlined above. The stations in the west of Langeness (T-3 and T-4) show the smallest variation in SPM and the weakest dependency on the prevailing wind direction. At station T-4, however, the highest density of data is recorded at on average twice as high concentrations compared to station T-3. In case of long lasting easterly winds, the higher elevated stations T-5 and T-6 in the north are characterized by even lower water depths as these winds counteract the rising tide. This results in a similar data pattern at both stations characterized by few data but with on average higher SPM concentrations at station T-6 (Figure 2-12). To take a closer look at these different patterns, we now consider the relations of SPM concentration to increasing wind speeds. Therefore, linear regression analysis is performed between wind speed 
and SPM concentration separated for the wind direction quadrants ( $N$ to $E, E$ to $S, S$ to $W, W$ to N) (Figure 2-13). The four individual inundation events (Table 2-4) are treated separately.

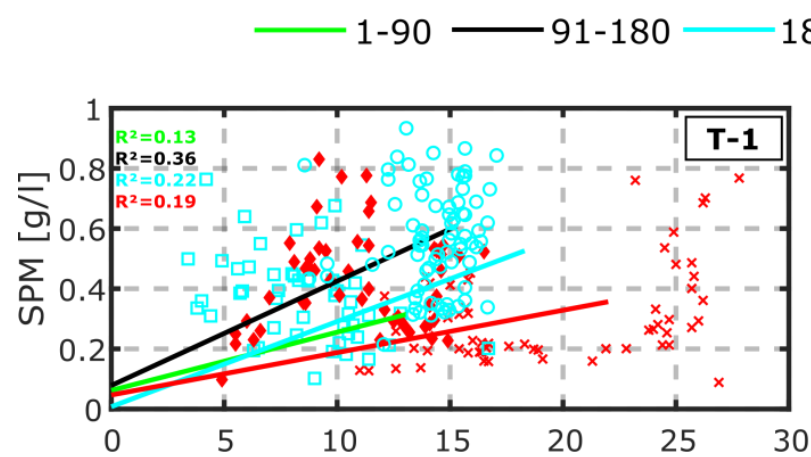

81-270 271-360 wind direction [degree] 1.× 2. $\square$ 3. 4.0 inundation event
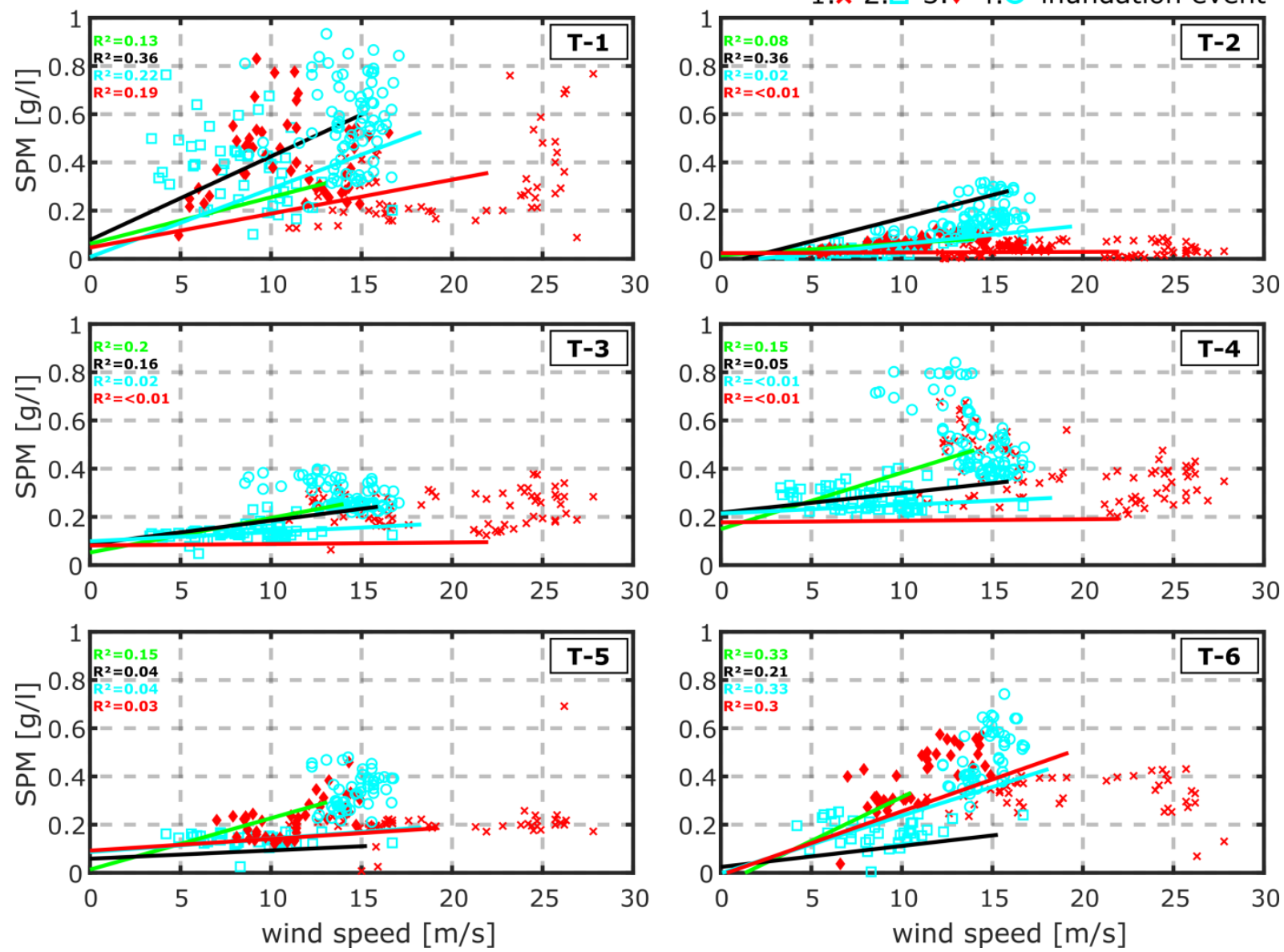

Figure 2-13: Linear regression analysis between wind speed and SPM concentrations, separated for the different wind direction quadrants, during the measured winter period from October 1, 2017 to February $15,2018 . R^{2}$ is the coefficient of determination after Pearson. The four individual inundation events (Table 2-4) are marked with different symbols and colour coding according to the associated wind direction quadrant. Inundation event 3 was not recorded by stations T-3 and T-4 due to technical issues.

The regression results of the spatial related stations show striking similarities: station T-2 shows an attenuated form of station T-1, T-3 an attenuated form of T-4 and T-5 an attenuated form of T-6. The sensitivities to higher SPM concentrations at increasing wind speeds, however, are different with respect to wind direction and station location. For instance, the stations in the south (T-1, T-2) show the highest SPM increase in the second quadrant between 91 and 180 degree wind directions and the lowest in the fourth quadrant between 271 and 360 degrees. The stations in the north $(T-5, T-6)$ show the highest SPM increase in the first quadrant between 1 and 90 degrees, i.e. wind direction from north to east. A similar pattern is observed for the stations in the west (T-3, T-4). 
During storm surges leading to inundations, SPM concentrations differ between individual events and stations (Figure 2-13). Regarding wind speed, the first inundation was the strongest (see Figure 2-11 box 1 and Table 2-4) but the SPM concentrations are not correspondingly high. Instead, the SPM concentrations roughly follow the mostly low slopes for the respective wind direction of event 1 (Figure 2-13, Table 2-4).

Table 2-4: Key parameters averaged over four tidal cycles within four different storm surge events leading to inundation of Langeness. Ten minutes averaged mean wind speeds and directions from the weather station Strucklahnungshörn and ten minutes averaged mean significant wave heights from the sea state buoy Süderaue.

\begin{tabular}{lllll}
\hline Inundation event & $\begin{array}{l}\text { wind speed } \\
{[\mathrm{m} / \mathrm{s}]}\end{array}$ & $\begin{array}{l}\text { top wind } \\
\text { speed }[\mathrm{m} / \mathrm{s}]\end{array}$ & $\begin{array}{l}\text { wind direction } \\
\text { [degree] }\end{array}$ & $\begin{array}{l}\text { significant wave } \\
\text { height }[\mathrm{m}]\end{array}$ \\
\hline 1. (29.10.2017) & $18.8 \pm 5.1$ & 27.8 & $329.3 \pm 18.4$ & $0.96 \pm 0.32$ \\
2. (08.12.2017) & $8.1 \pm 2.6$ & 16.7 & $268.9 \pm 19.8$ & $0.41 \pm 0.11$ \\
3. (04.01.2018) & $10.7 \pm 3.8$ & 18.1 & $280.6 \pm 8.7$ & $0.48 \pm 0.19$ \\
4. (12.02.2018) & $14.2 \pm 1.7$ & 17.1 & $243.3 \pm 11$ & $0.69 \pm 0.11$
\end{tabular}

The other inundation events 2, 3 and 4 occurred during mainly westerly wind directions (243 $281^{\circ}$; Table 2-4), among which event 4 had the highest wind speed. The southwestern wind direction $\left(243^{\circ}\right)$ matches the main wind directions during the recorded winter period (Figure 2-11) and shows the overall highest SPM concentrations at all stations in the tidal flat (Figure 2-13). At the stations where all these three events were recorded, decreasing average wind speed correlates with decreasing average SPM. Stations T-2, T-3 and T-5, which show generally lower SPM concentrations, also show relatively smaller SPM increase during inundation events.

\subsubsection{Spring time series}

SPM concentrations are generally lower in the spring period compared to the winter period (Figure 2-14), as well as the recorded wind speed (Figure 2-11). No data for stations T-5 and T-6 is displayed because the small number of measurements due to low water level at these stations. Nevertheless, even in the measured spring period, stronger winds can significantly increase the SPM concentration as observed during few days with stronger wind (May 1 to 4, 2017; Figure 2-11, box 2). The average wind speed reached $9 \pm 2 \mathrm{~m} / \mathrm{s}$ at rather stable wind direction $\left(81 \pm 12^{\circ}\right)$. During these stormy winds the average SPM concentrations exceeded the 
average SPM concentrations over the entire spring period by factors of 3.5 (T-1), 2.5 (T-2), 2.0 ( $T-4)$ and 1.7 (T-3). These elevated SPM concentrations are visible in Figure 2-14, wind direction between 70 and 90 degree. This spring storm data highlight that longer-lasting and stable winds can lead to a significant increase in SPM concentration even during spring time.

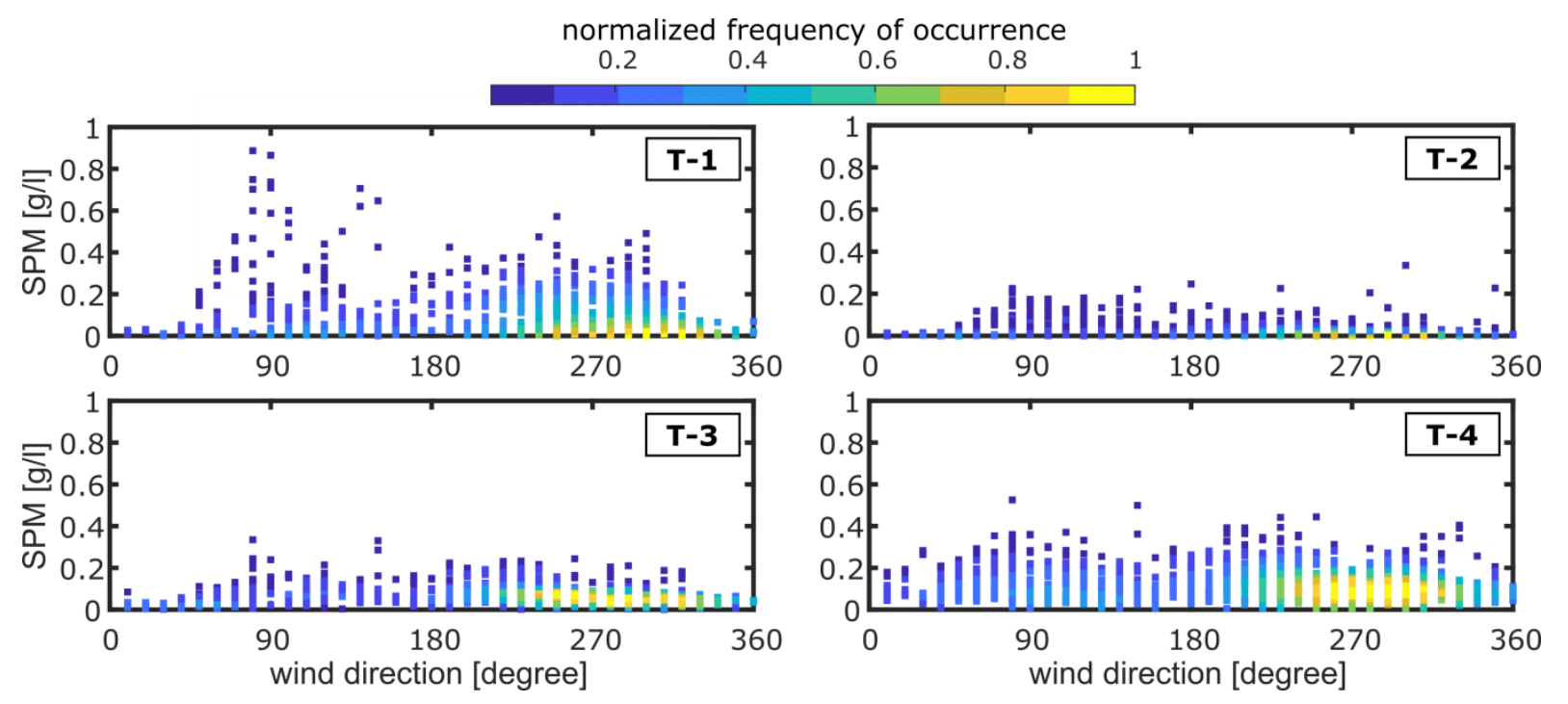

Figure 2-14: Density plot of SPM concentrations versus wind direction at stations T-1, T-2, T-3 and T-4 measured during the same spring period as in Figure 2-11, from February 25, 2017 to June 1, 2017.

\subsubsection{Spatial SPM variability}

In order to obtain an overall impression of the SPM distribution in the study area, the determined SPM concentrations of the different time series were averaged. SPM concentrations were summed up and divided by the respective number of measurements at the individual station to calculate the mean (Figure 2-15). This mean SPM was highest over the entire winter period at stations T-1 and T-4, followed closely by T-6. The lowest SPM concentrations are captured at station T-2. The mean winter SPM could be at least doubled at all stations during inundation event no. 4. The magnitude of the mean SPM increase of the respective stations follows the same ascending order as already observed (T-2, T-3, T-5, T-6, T$4, T-1$ ) in the investigation of the two tidal cycles under different weather conditions (Figure 2-9). This order persists within a time series of several months during the winter period. The biggest differences in mean SPM are observed between the stations T-1 and T-2 in the south of Langeness. The mean SPM is about four times higher at station T-1 and up to more than seven times higher during storm conditions. Therefore, the potential for generating higher SPM concentrations even under harsh weather conditions seems to be strongly limited in the area of station T-2. In the west of Langeness, station T-4 shows on average about twice as high SPM concentrations during the entire winter season as the neighboring station T-3, a trend that 
persists even during the inundation events. In the north of Langeness, station T-6 is characterised by higher mean SPM compared to T-5, especially during inundation events.

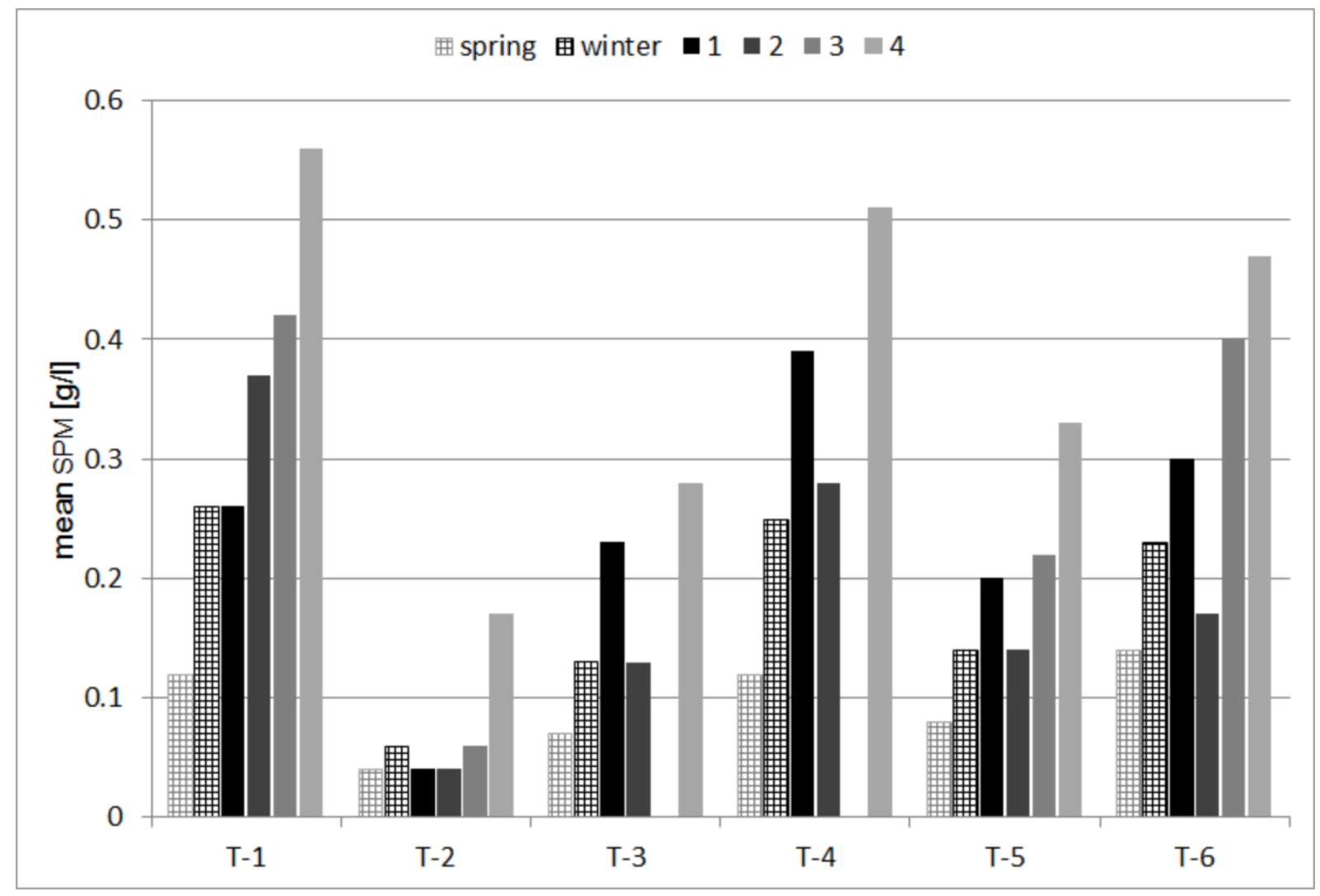

Figure 2-15: Bar plot of mean SPM concentrations of the measured spring period from 25th February 2017 to 1st June 2017, the winter period from October 1, 2017 to February 15, 2018, and the individual inundation events numbered from 1 to 4 as in Table 2-4. The third inundation event was not recorded at stations T-3 and T-4 due to technical issues.

\subsection{Discussion}

\subsubsection{Calibration}

Benns and Pilgrim (1994) showed experimentally that the OBS response increases with decreasing particle size between $12 \mu \mathrm{m}$ and $212 \mu \mathrm{m}$. This general observation is strongly supported by our results (Figure 2-7). At every calibration run, with increasing SPM concentration inside the calibration tank, the signal to noise ratio increased, which in turn led to an increased standard deviation during recording the OBS response. This behaviour has already been investigated by Downing and Beach (1989) and Black and Rosenberg (1994) who 
explained this by variations in the instantaneous sample concentration inside the calibration tank.

To evaluate the quality of our laboratory calibration, individual reference measurements were carried out for each calibration run, which were evaluated separately. These reference measurements provide a good opportunity to check the calibration results for reproducibility, which in our case is very convincing, even for low concentrations $<0.2 \mathrm{~g} / \mathrm{l}$ (Figure 2-7). The comparison with the in situ samples showed a satisfying agreement with the laboratory calibration results (Figure 2-8).

An obvious drawback of using accumulator sediments for calibration is the long sampling time until enough sediment is collected, especially at higher elevated stations which are less inundated and have lower collection times, i.e. stations T-5 and T-6. However, we decided, not to add any local bed sediment to the accumulator samples to cover the remaining calibration interval (i.e. $\sim 150 \mathrm{mV}$ at T-5 and $\sim 200 \mathrm{mV}$ at T-6; Figure 2-7) because the large differences in PSD between bed sediment and accumulator sediments (Table 2-3) would inevitably distort the calibration. Because of the linear relationship in all our calibration runs between OBS response and SPM concentration, we are confident that the linear extrapolation still provides reliable results beyond the end of the calibrated data range $(0.38 \mathrm{~g} / \mathrm{l}$ at T-5 and $0.5 \mathrm{~g} / \mathrm{l}$ at T-6; Figure 2-7). We are aware that even the well-suited accumulator calibration samples cannot achieve an exact solution in quantifying the SPM concentration in the field, especially for longer periods with fluctuating hydrodynamic conditions. Therefore, calibration runs and comparisons with in situ samples should be performed consistently. Nevertheless, the in situ results clearly showed that the accumulator sediments are suited for OBS calibration. We therefore recommend the type of calibration used here, especially if the permanent in situ sampling at a variety of measurement stations is not possible for logistic, safety and cost reasons.

\subsubsection{Field measurements}

The results of this study demonstrate the variability of SPM concentrations at different time scales, weather conditions, seasons and local conditions (e.g. water depth, proximity to tidal channels) and anthropogenic influences (e.g. protection measures) on the tidal flats around Langeness. Already the comparison of two tidal cycles under fair weather and storm conditions showed a clear shift towards higher SPM concentrations during storm conditions. The SPM concentrations during storms can be several times higher as the concentrations under fair weather conditions. Within several tidal cycles, we have noted further fluctuations in SPM 
concentration (Figure 2-10). The SPM concentration was highest at the mid-tide and lowest at slack water conditions during high and low tides were sediment settling takes place, which can rapidly and significantly reduce SPM concentration. The SPM decrease during low tide was only interrupted at low water levels when wave induced resuspension of bed sediment occurred even at lower wave heights. This is consistent with results of a qualitative model by Gätje and Reise (1998) within a 2 m deep region located to the north of the island of Föhr, which shows higher bed shear stresses induced by sea state compared to the tidal current at moderate wind speed of $5 \mathrm{~m} / \mathrm{s}$. These fluctuations were maintained during storm surges, but at higher SPM concentration ranges. These observations during tidal cycles are consistent with other studies (e.g. Ridderinkhof et al. 2000; Andersen and Pejrup 2001; Bartholdy and Aagaard 2001; Bartholomä et al. 2009; Badewien et al. 2009).

During one inundation event we analyzed mean SPM concentrations that were nearly two times the averaged concentration during the whole winter period of more than four months. Between the individual stations distinct contrasts in the SPM increase are observed. A closer look at the individual inundation events indicates that these events also vary considerably depending on the prevailing wind conditions and station location. In general, SPM concentrations were higher in case of persistent strong winds from westerly directions, which correspond to the most frequent wind directions during the whole winter period. For three inundation events under westerly wind directions $\left(243-281^{\circ}\right)$ the mean SPM concentration increased with increasing average wind speed.

During inundation event 1 with a northerly wind direction, the SPM concentration was lower despite the highest wind speeds, which underlines the strong influence of the wind direction on an overall increased SPM concentration for the study area. However, the number of observed inundation events is too low to cover all the relevant variables and therefore further measurements during inundation events are required. The highest SPM concentrations at all stations in the tidal flats were obtained during the inundation event no. 4 with a southwesterly wind direction with averaged wind speed of $14.2 \mathrm{~m} / \mathrm{s}$ in combination with an incipient spring tide. Although we cannot yet determine the exact impact of a spring tide on an SPM increase during a storm, higher SPM concentrations during a spring tide due to higher flow velocities have already been reported by, e.g., Bartholomä et al. (2009). We therefore hypothesize that a 
superposition of a stable wind direction from SW, high average wind speed and spring tide meets the optimal conditions for high SPM concentrations in the study area.

In the spring period, average SPM concentration and variability are lower at all stations in the tidal flat. This results from lower average wind speed with consequently lower hydrodynamic activity compared to the winter months. Measured SPM differences are thus largely controlled by regional meteorological patterns. This finding is in accordance with other studies (e.g. Fettweis et al. 2006; Fettweis et al. 2007; Fettweis et al. 2012). Nevertheless, in rare cases higher SPM concentration were determined during stronger winds blowing for several days from a constant direction. This effect was also observed by Badewien et al. (2009) in a tidal inlet between the East Frisian Islands of Langeoog and Spiekeroog (southern North Sea). They found that long-lasting wind have the same or even larger impact on SPM concentrations than a single storm surge event in their area. The overall SPM potential during the measured spring period, however, remained rather low and is therefore no longer considered.

\subsubsection{Spatial SPM availability and low spots}

All stations are able to respond to favorable wind conditions with higher SPM concentrations unless they are constrained by man-made constructions like high spur dykes. Depending on the location and the prevailing wind conditions, a temporally and spatially variable SPM distribution is observed in the tidal flats of the study area.

For the stations in the west of Langeness $(T-3, T-4)$, no distinct trend is found relating SPM concentrations with increasing wind speed. The comparatively poor correlation results (Figure 2-13) and the most horizontally elongated data density maxima in Figure 2-12 can be interpreted as a predominant influence of tidal currents with a lower sensitivity to changing wind conditions which is consistent with the observations of Gätje and Reise (1998). The most likely reasons are higher water depths in this area and consequently relatively less wave energy input on the bed sediments forcing resuspension especially at storm surge conditions with above-average water depths. Therefore, significantly higher SPM concentrations as determined during the inundation events 1 and 4 at station T-4 are preferentially achieved around midtides when tidal currents are more active, especially close to a tidal channel. During the fourth inundation event high SPM concentrations were also detected during low tide at station T-4. Due to harsh weather conditions, we assume that long-lasting stable wind conditioned wave activity led to this SPM increase (Figure 2-10). The station T-3 shows similar pattern in an attenuated form because tidal currents decrease with increasing distance to the tidal channel 
and consequently the capacity to keep particles in suspension decreases, too. This results in a lower data density maximum and generally lower y-intercepts of the regression lines as compared to station T-4 (Figure 2-12, Figure 2-13). We therefore suggest that advection of suspended material by the tidal channels Süderaue and Norderaue is the main controlling factor for increasing SPM concentrations in these lower-lying western areas during the high water levels.

In contrast, two of the higher-lying areas within our measurement network (T-1 in the South and T-6 in the North) are characterized by strong positive correlation of increasing SPM with increasing wind speed. Both areas have relatively shallow water depth which favors resuspension triggered by harsh sea state conditions. At station T-6 the nearby land reclamation area without spur dykes may also serve as an additional source for fine sediment. This assumption is supported by the fact that the strong positive correlation described above is more independent of the wind direction (Figure 2-13). In comparison to the tidal flats at station T-6 the water level in front of station T-1 deepens faster (Figure 2-2). This could favor a stronger energy input of larger waves propagating from deeper regions to the shallower water, which would favor resuspension of bed sediments next to the measurement position. In addition, this area appears to be affected by the prevailing flow conditions of the tidal channel Süderaue, as the observed variations are particularly high at the inflection points of the tidal cycles where the tidal current is fastest. The highest determined SPM variabilities within our turbidity network at station T-1 could therefore be the result of the superposition of resuspension next to the station and advection transport of fine sands to the station which are more frequent in the channel beds of Süderaue compared to Norderaue (e.g. Asmus et al. 2016).

Within the turbidity network spots of remarkable low SPM concentrations could also be located. The most obvious low spot is the southwestern area at the low-lying station T-2. The data clearly show that the constricted position between the spur dykes has a significant attenuating effect on the hydrodynamic conditions in the area causing (i) usually low SPM concentrations and (ii) the finest PSD of the bed sediment in the study area (Table 2-3). Higher SPM concentrations were only recorded in a small range of wind directions, which fit to the direction of the opening between the two spur dykes. Therefore, the SPM concentrations at station T-2 remain moderate even during inundation conditions. In contrast, SPM is usually highest at more exposed stations like T-4, which is additionally in the vicinity of a tidal channel. 
The higher SPM availability at stations T-1, T-4 and T-6 compared to the lower SPM availability at stations $\mathrm{T}-2, \mathrm{~T}-3$ and $\mathrm{T}-5$ results in a heterogeneous SPM distribution between the western and eastern parts of Langeness.

\subsection{Conclusion and outlook}

To our knowledge we are the first to successfully run an autonomous operating network of stationary turbidity measuring stations around an island-like salt marshland, located in the tidal flats of the North Frisian Wadden Sea (Southern North Sea). Based on this network, we have gained new insights into variability of SPM concentrations at different time scales which range from a single tidal cycle up to several months.

The comparison of SPM concentrations between fair and harsh weather conditions offers insights into the spatial distribution of SPM in the study area. Stations located within an area with relatively free access to a tidal channel show on average more than twice as high SPM concentrations during fair weather conditions. All stations show higher SPM concentrations during harsh weather conditions. The highest SPM availability around Langeness was recorded during a storm surge with an average wind speed of $14.2 \mathrm{~m} / \mathrm{s}$ from a southwestern direction. The strongest storm (average wind speed $\sim 19 \mathrm{~m} / \mathrm{s}$ ) does not trigger the highest SPM concentrations, indicating that wind direction is also highly relevant. SPM concentrations measured at stations that are higher in altitude due to the tidal flat bathymetry depend more on wind speed and direction than at low-lying stations. The clearest low spot in SPM availability was determined in a low-lying area between two spur dykes. The observed patterns prove a persistent spatially and temporally inhomogeneous distribution of SPM around Langeness.

The next steps will include (i) the analysis of single inundation events considering the SPM from the ongoing measurement campaign, (ii) the analysis of more events under storm conditions to better constrain the relevance of individual controlling factors of SPM availability, (iii) comparing the temporal and spatial variability of SPM measured on the intertidal flats with sediment accretion rates on different parts of the marshland (Hallig Langeness), and (iv) using the results to validate a numerical sediment transport model for Langeness and the surrounding flats, which is designed to test scenarios for an optimized coastal protection. 


\section{Acknowledgements}

The study forms part of the joint research project "Real life coastal laboratory to develop shortand long term strategies for the protection and preservation of Schleswig-Holsteins Halligen (Living Coast Lab Hallig)" funded by the German Federal Ministry of Education and Research (BMBF, 03F0759C).

We thank the inhabitants of Langeness for their hospitality and for supporting the research project. We thank the Landesbetrieb für Küstenschutz, Nationalpark und Meeresschutz Schleswig Holstein (LKN.SH) for their accomplished altitude measurements of all stations within the network, for their help in installation and maintenance of the turbidity network and for providing topographical, sea state and gauge level data. We thank the Bundesamt für Seeschiffahrt und Hydrographie (BSH) for providing nautical data and the Deutscher Wetterdienst (DWD) for providing wind data. We further want to thank Caren Stachorra for continuous help in the field and laboratory as well as Artiom Patrinica from AVP-Systeme for helping us networking our whole turbidity measurement network. We highly acknowledge detailed and constructive advice by two anonymous reviewers as well as careful editorial handling by Augustin Sanchez-Arcilla. 


\section{Suspended particulate matter for sediment accumulation on inundated anthropogenic marshland in the southern North Sea - Potential, thresholds and limitations}

This paper is published 2020 in Continental Shelf Research, 207 (2020), 1-14

Doi: https://doi.org/10.1016/j.csr.2020.104214

Ingo Hache, Volker Karius, Hilmar von Eynatten

Corresponding author: Ingo Hache, University of Göttingen, Geoscience Center, Goldschmidtstr.

3, 37077 Göttingen, Germany, ingo.hache@uni-goettingen.de

Geoscience Center University of Göttingen, Department of Sedimentology and Environmental Geology, Goldschmidtstraße 3, 37077 Göttingen, Germany

Keywords: Suspended particulate matter, Sediment transport, Inundation, Vertical accretion, Sea level rise, Marshland 


\section{Abstract}

Determining the availability of suspension load, i.e. suspended particulate matter (SPM), in shallow marine tidal systems is necessary to estimate whether coastal lowlands may experience vertical accretion rates that keep pace with global sea level rise. In particular anthropogenic marshland areas are affected by limitations of SPM accumulation due to artificial structures such as dikes and revetments that act as transport barriers.

With the use of an autonomously operating turbidity measuring network installed at and around the Hallig Langeness in the North Frisian Wadden Sea, we have analyzed eight individual inundations on this island-like marshland during harsh weather conditions. The results reveal that the majority of the available SPM could not accumulate on the marshland due to adjacent coastal protection measures. This reduction in SPM affects especially grain sizes within the very fine to fine sand range between 63 and $200 \mu \mathrm{m}$ that can account for up to $70 \%$ of the sampled SPM during an inundation. Furthermore, we have determined a tidal average SPM concentration threshold of $0.15 \mathrm{~g} / \mathrm{l}$ should be available on the tidal flat in order to allow sufficient vertical marshland accretion. Although this threshold is exceeded by the determined SPM on the tidal flats around Langeness, the available SPM on the marshland after passing the coastal protection measures is at most $20 \%$. Hence, an optimized coastal management strategy has the potential to increase the sediment accumulation rate on the marshlands by a factor of five, which might allow for keeping pace with local sea level rise.

\subsection{Introduction}

Global and regional sea level rise (SLR) affects the conservation and natural adaptation capacity of intertidal and especially supratidal areas, which is why protection and utilization of these areas has become a major research issue (Kirwan et al. 2010, 2016a, 2016b; Kirwan and Megonigal 2013; D'Alpaos et al. 2011; Schuerch et al. 2012, 2013; Schindler et al. 2014a, 2014b; Horton et al. 2018). Among highly dynamic tidal areas like the tidal flats of the southern North Sea, there are the so-called Halligen, ten small island-like marshlands with a total area of approximately $23 \mathrm{~km}^{2}$ and an average elevation of only a few decimeters above the mean high water (MHW) in the North Frisian Wadden Sea Figure 3-1. 


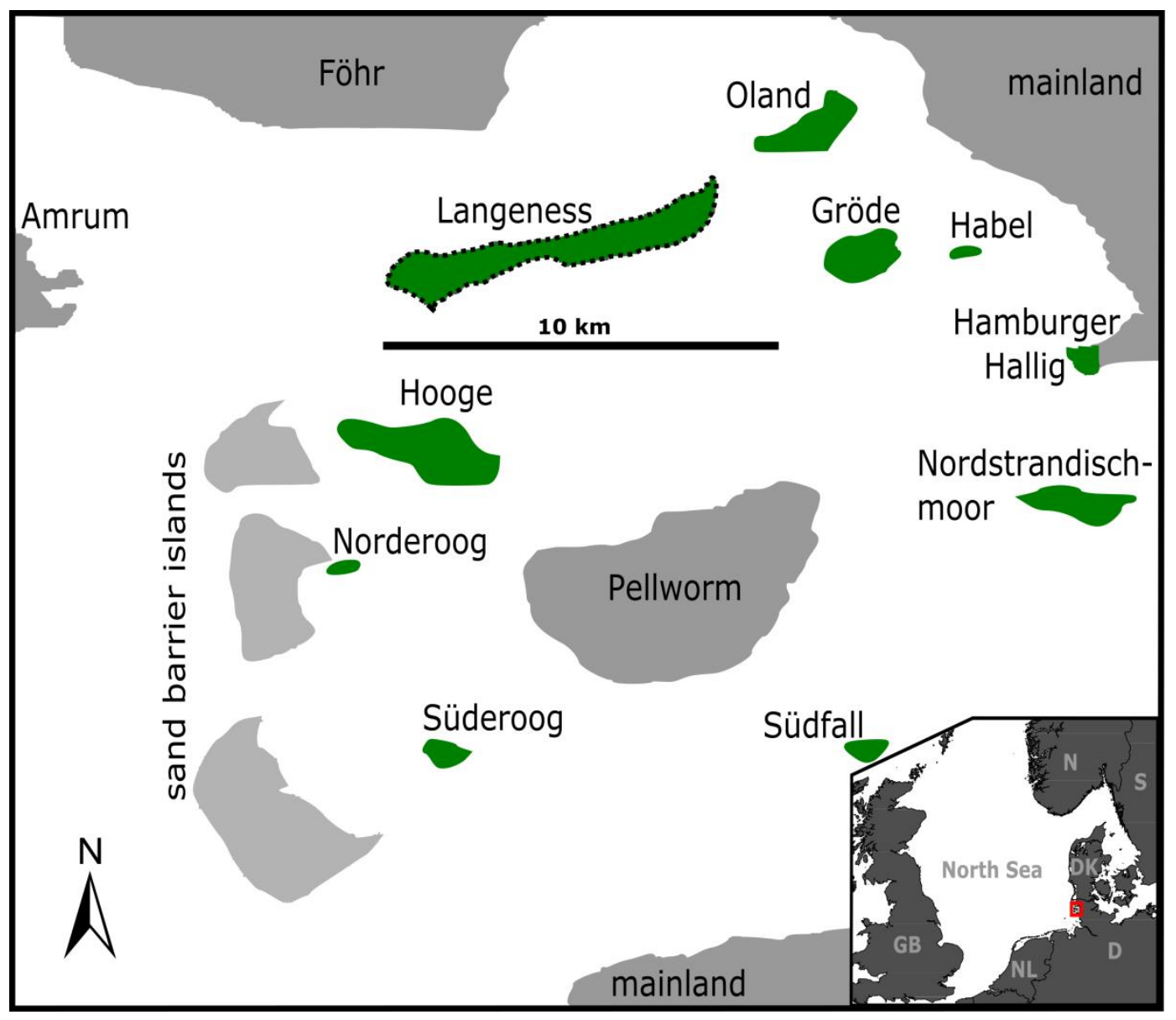

Figure 3-1: The map shows the ten Halligen (green), located in the North Frisian Wadden Sea in the southern North Sea. The study area Langeness (black dotted outline) is surrounded by the islands of Föhr, Amrum and Pellworm and the mainland of Schleswig Holstein as well as several other Halligen and three sand barrier islands. The inset shows the location of the area within the North Sea and its neighboring countries: $\mathrm{GB}=$ Great Britain, $\mathrm{NL}=$ The Netherlands, $\mathrm{D}=$ Germany, $\mathrm{DK}=$ Denmark, $\mathrm{S}=$ Sweden, $\mathrm{N}=$ Norway.

Unlike the surrounding islands, the Halligen have no consolidated nucleus formed by Pleistocene glacial deposits; they consist largely of Holocene tidal sediments which make them more prone to erosion. The so-called 1st and 2nd Grote Mandränke in 1362 and 1634, respectively, were the most catastrophic storm surge events in historical time where thousands of people were killed. The latter event created the last huge land loss in the North Frisian Wadden Sea (Reise 2005) and gave the Halligen their current isolated appearance (Ahrendt 2007) as remnants of the former coherent marshlands. However, the remaining Halligen have still lost between 50 and 80 percent of their land mass (on average) since the first survey in 1713 until the authorities started to stabilize the Hallig margins around 1900 (Quedens 1991). Protection measures started with small dyke constructions in 1914 on the two largest Halligen Hooge and Langeness (Müller and Fischer 1917) and evolved to distinct protection measures 
like breakwater constructions and revetments which today entirely enclose at least the larger Halligen. Nevertheless, the Halligen are massively affected by storm surges, extreme weather conditions and the increasing SLR (Deicke et al. 2007).

The measures provide certain protection against erosion by tidal currents and storm induced waves during inundations. However, conventional coastal protection measures can block the wave- and wind-driven supply of sediments along coastlines (e.g. Temmerman et al. 2013), which likely applies to the Halligen as well. As a consequence, the vertical accretion of such marshland surfaces is limited, and depends on storm frequency and intensity (e.g. Schuerch et al. 2013). None of the present vertical accretion rates measured on three of the larger North Frisian Halligen at $1.5 \pm 0.9 \mathrm{~mm} / \mathrm{a}$ for Hooge, $2.6 \pm 0.9 \mathrm{~mm} / \mathrm{a}$ for Nordstrandischmoor and $1.2 \pm$ $0.8 \mathrm{~mm} / \mathrm{a}$ for Langeness (Schindler et al. 2014a) can compete with the observed annual local MHW rise of $5.0 \pm 0.3 \mathrm{~mm} / \mathrm{a}$ (1951-2011; Schindler et al. 2014a), neither with the average estimated global mean sea level (MSL) of $3.4 \pm 0.4$ mm/a (1993-2009; Nerem et al. 2010) with an climate-change-driven acceleration of $0.084 \pm 0.025 \mathrm{~mm} / \mathrm{y}^{2}$ during the last 25 years (Nerem et al. 2018) or the local MSL increase in the inner North Sea of $4.6 \pm 1.8 \mathrm{~mm} / \mathrm{a}$ (1993-2009; Wahl et al. 2013). Moreover, model based investigations of 65 extreme storm events between 1970 and 2009 by Arns et al. (2015a) show that an added MSL of +0.54 m causes an additional dramatic local storm surge water level increase of up to $+0.15 \mathrm{~m}$ in the shallow water areas of the German Wadden Sea.

The natural adaptation capacity of the supratidal flats in the North-Frisian Wadden Sea is strongly influenced by the availability of suspended particulate matter (SPM) in the intertidal flats during harsh weather conditions, resulting in vertical accretion on the supratidal marshland (i.e. the percentage of available SPM that is delivered onto the marshland and subsequently settled).

SPM was examined on the tidal flats around Hallig Langeness and on the marshland of the Hallig itself during two winter seasons (2017/18 and 2018/19) for eight individual inundation events each caused by a single tide. This SPM examinations were conducted using a unique autonomous working turbidity measurement network (Hache et al. 2019). The main question to be answered is whether the sediment accretion of the Hallig during inundations is material limited (i.e. there is not sufficient SPM available in the water column around the Hallig) or transport-limited (i.e. SPM is available but not transported efficiently onto the Hallig). 
In this study, we present the results of determined SPM in combination with sediment accumulation and accretion rates of eight individually investigated inundations. The results reveal the conditions allowing for high SPM availability on the tidal flats, as well as high sediment accumulation on the marsh surface. We also determine a minimum SPM availability threshold on the tidal flat that would be necessary to keep pace with the rising MHW. Furthermore, we demonstrate the impact of the existing coastal protection measures on present sediment accumulation rates. The study was carried out within the interdisciplinary joint research project "Living Coast Lab Halligen", which is intended to develop strategies to mitigate the imbalance between SLR and vertical accretion.

\subsection{Material and methods}

\subsubsection{Study area}

Hallig Langeness is located in the Nord Frisian Wadden Sea off the coast of the federal state of Schleswig-Holstein (Germany) and is the largest of the ten North Frisian Halligen with a total area of $9.24 \mathrm{~km}^{2}$. Harsh weather conditions are especially common in the winter months, and often lead to storm surges in this area, which inundate the entire Hallig Figure 3-2a, Figure 3-2b. The 113 inhabitants (2018) protect themselves, their cattle and goods against these inundations by living on man-made dwelling-mounts (called "Warfen") which are on average $5.06 \pm 0.45 \mathrm{~m}$ above MSL (Figure 3-2b). For the best possible protection of the inhabitants the Warfen were heightened in 2000 and further increases are already implemented due to the continuous MHW rise (Hofstede 2019). Without anthropogenic structures, the terrain height of Langeness has an average absolute ground level of $0.17 \mathrm{~m}$ above $\mathrm{MHW}$; protection measures like dykes and revetments encompass the entire Hallig (Figure 3-2c) with an average height of $0.98 \mathrm{~m}$ above MHW (Schindler and Willim 2015) to protect it from erosion and to avoid minor inundations. To reduce the wave energy on the marsh surface at high water levels, water permeable rubble breakwater constructions were partially installed along the edge of Langeness (Figure 3-2c). To protect the marshland against flow induced erosion, spur dykes have been installed to keep the tidal channels Norderaue (to the north of Langeness) and Süderaue (to the south of Langeness) at some distance from the edge of Langeness (Figure 3-2d). The tide is semidiurnal with an average mean tidal range of $2.7 \mathrm{~m}$ measured at tide 
gauge Hilligenley (Figure 3-3) during the winter measuring periods from October 1, 2017 to February 15, 2018 and from September 1, 2018 to March 01, 2019. Such range is characteristic for mesotidal regimes according to Hayes (1979).

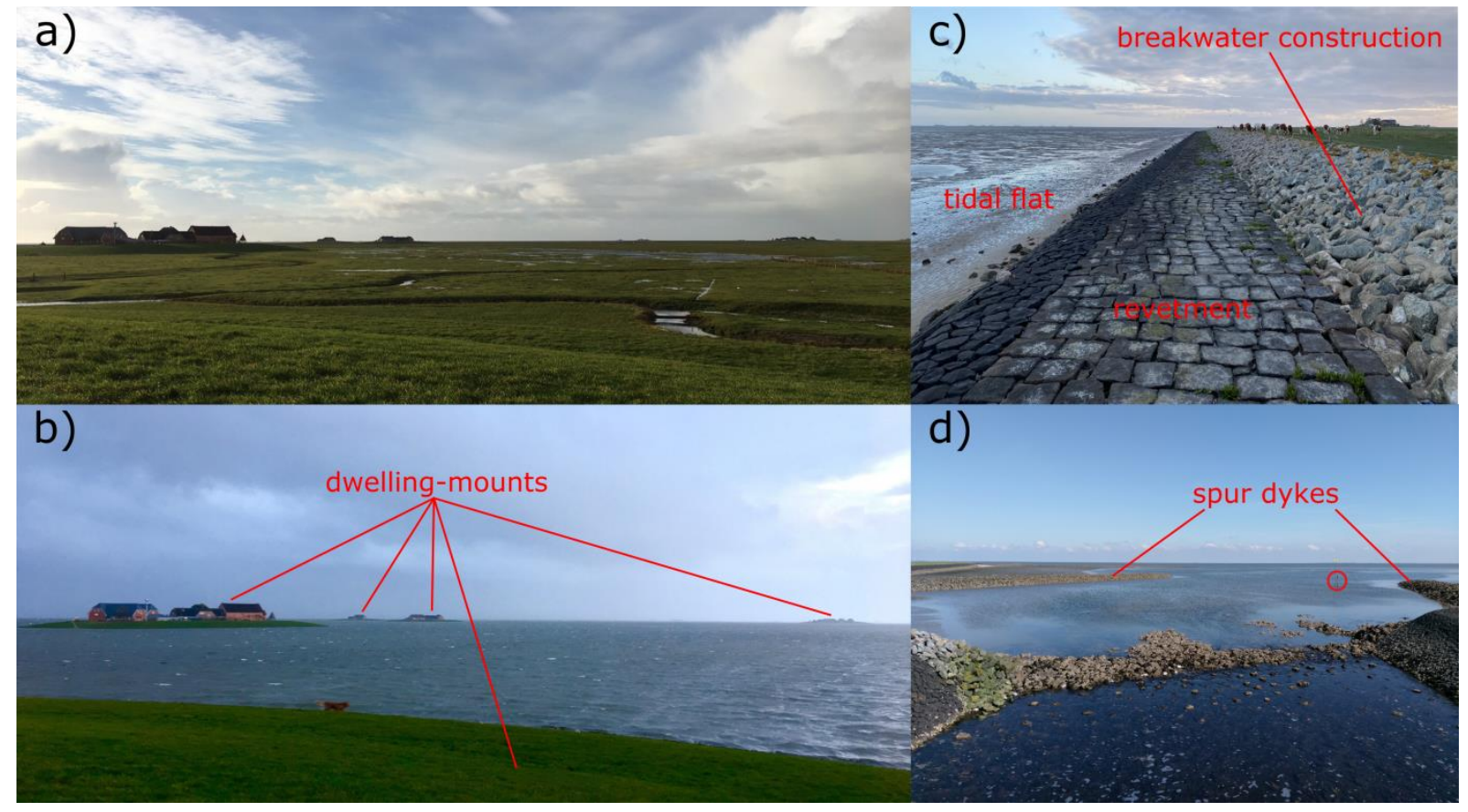

Figure 3-2: (a) Impressions of the vegetated landscape of Langeness showing the flat marshland and dwelling mounts with houses (b) same view as in (a) but during an inundation. (c) Example of the revetment and breakwater construction. (d) The spur dykes enclosing the turbidity measurement station T-2 (circle) in the southwest of Langeness (see Figure 3-3).

The sediment grain sizes on the tidal flats around Langeness were investigated in a survey covering every $100 \mathrm{~m}$ within $500 \mathrm{~m}$ long transects perpendicular to the shore line. 1280 samples were analyzed by finger tests and 113 of them verified by laser particle sizer, using the classification of Folk and Ward (1957). The results revealed a mixed sediment flat with predominantly fine sand along with a small increase of the grain size towards the tidal channels. Only a small area in the south revealed a tidal flat with finer grain size distribution, i.e. a higher proportion of silt-sized material. Grain size analysis of the tidal channels by Asmus et al. (2016) showed medium sand as the dominant grain size in the tidal channel Norderaue. These medium sands also occur in deeper regions in the tidal channel Süderaue whereas the shallower levels are dominated by fine sand. 


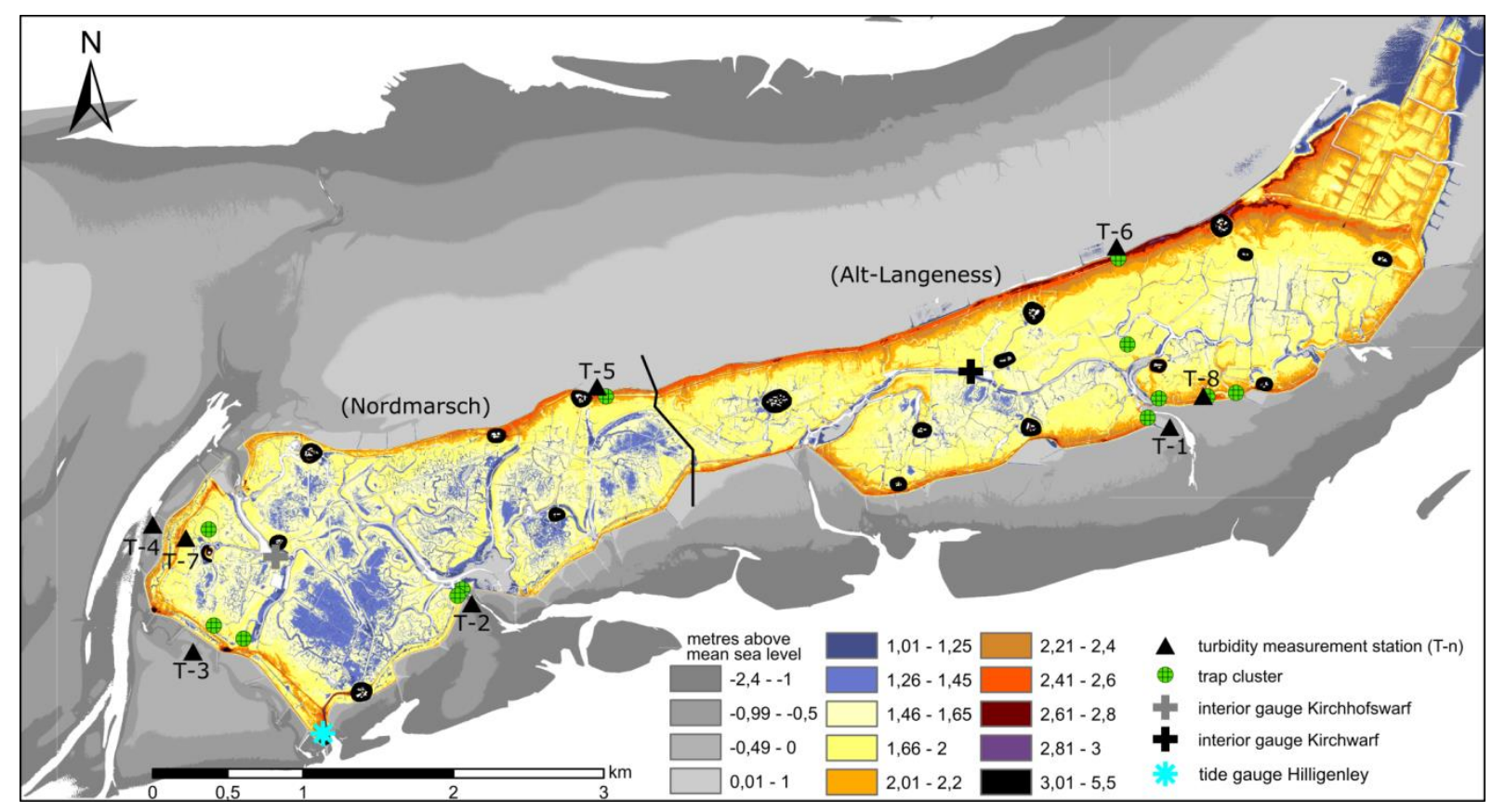

Figure 3-3: Digital ground model of Langeness (based on Laser scan DGM1 data from 2014, courtesy of LKN.SH). The black line divides Langeness in its historical parts named Nordmarsch in the west and AltLangeness in the east. The white areas show depths that cannot be detected by laser scanning due to high water coverage in the two tidal channels Norderaue in the north of Langeness and Süderaue in the south. The black roundish areas are the dwelling-mounts.

\subsubsection{Turbidity measurement network}

The autonomously working network includes eight turbidity measurement stations (Figure 3-3) operating in the near-infrared wavelength range with an optical backscatter turbidity sensor OBS-3 + by campbell C scientific (Campbell Scientific 2017). Six stations are distributed on the tidal flats around Langeness (T-1 to T-6) and two stations are installed on the marshland of Langeness (T-7, T-8). The measurement duration of the stations depends on their elevation and related flooding frequency. The measurement cycle starts as soon as the OBS is permanently submerged by the rising water level with a measuring interval of 1 minute and immediately stops as soon as the water drops below the OBS sensor unit. Therefore, the stations on the tidal flats already measure during normal tides while the stations on the marsh surface are activated only during inundations. Next to each OBS sensor we mounted a self-built sediment accumulator (Design by $\mathrm{IM}+\mathrm{P}$ engineering office Dr.-Ing. Manzenrieder und Partner GbR http://www.imp-ol.de) to collect the sediments that were detected by the OBS. This sediment accumulator is called 'Base' while a second accumulator ('Top') was mounted approximately 1.2 $\mathrm{m}$ higher at each station to investigate the vertical sediment distribution within the water column during individual inundation events. We emptied the accumulators immediately before 
an inundation and sampled them as soon as the tidal flats were accessible again after the inundation. The sediment accumulators have a capacity of approximately $350 \mathrm{~cm}^{3}$, six inlets of $8 \mathrm{~mm}$ in diameter and a closed top to minimize resuspension of the sample inside the accumulator. With an aspect ratio of 3.6 the accumulator fulfills the recommended aspect ratio conditions by Gardner (1980a; 1980b) to prevent resuspension at higher flow velocities. Furthermore, each turbidity measurement station received an individual calibration with the sampled materials from the respective Base sediment accumulator to convert the OBS signal from millivolt to grams per liter. For the calibration process, the accumulator samples were wet sieved to filter out seagrass and shells and were subsequently stirred with an electric paint stirrer with sufficient water to get a homogeneous suspension. While stirring, aliquots were extracted from the suspension and transferred into a self-built calibration tank in which the suspension was circulated to ensure a homogenous distribution. After each added aliquot the OBS response inside the tank was recorded for 60 seconds and averaged and assigned to the dried mass of solids. A more detailed description about the functionality and calibration procedure of the turbidity measurement system as well as the sediment accumulators is given in Hache et al. (2019).

\subsubsection{Sediment traps}

In addition to SPM the sediment accumulation and accretion rates on the Hallig were measured during individual inundation events with a well-tested combination of sediment traps, consisting of one-liter LDPE bottles (trap type A) and PE-synthetic turf mats (trap type B). The combined data from these traps are used to calculate the accretion rates, which is described in detail by Schindler et al. (2014b). The arrangement of the traps is illustrated in Figure 3-4a and further referred to as trap cluster. The bottles measure $20 \mathrm{~cm}$ in height from the bottom of the bottle to the base of the screw cap and $9.5 \mathrm{~cm}$ in diameter. The opening is five $\mathrm{cm}$ in diameter. The bottles are sunk into the ground up to the screw cap and filled with nearby brackish water in order to prevent them from floating in case of an incipient inundation. Next to the bottles the turf mats were fixed to the ground with five nails. Each turf mat has a surface area of 600 $\mathrm{cm}^{2}$ and is equipped with $2.1 \mathrm{~cm}$ long grass like PE-blades. Each trap cluster consists of five bottles and five turf mats. In total, we placed 12 trap clusters in the proximity of the turbidity measurement stations and some additional suitable locations (Figure 3-3). Locations were chosen with respect to different transport conditions, including the type and height of revetments and rubble breakwater constructions. Two trap clusters were placed each behind barriers of different height at three locations adjacent to stations $\mathrm{T}-1, \mathrm{~T}-2$, and $\mathrm{T}-3$. Note that 
the entire area of Langeness is covered with vegetation (Figure 3-2) and thus, all trap clusters have been installed on vegetated areas. Figure 3-4 shows a setup of all measurement devices that were used on the marshland including sediment traps, sediment accumulators and a turbidity measurement station.

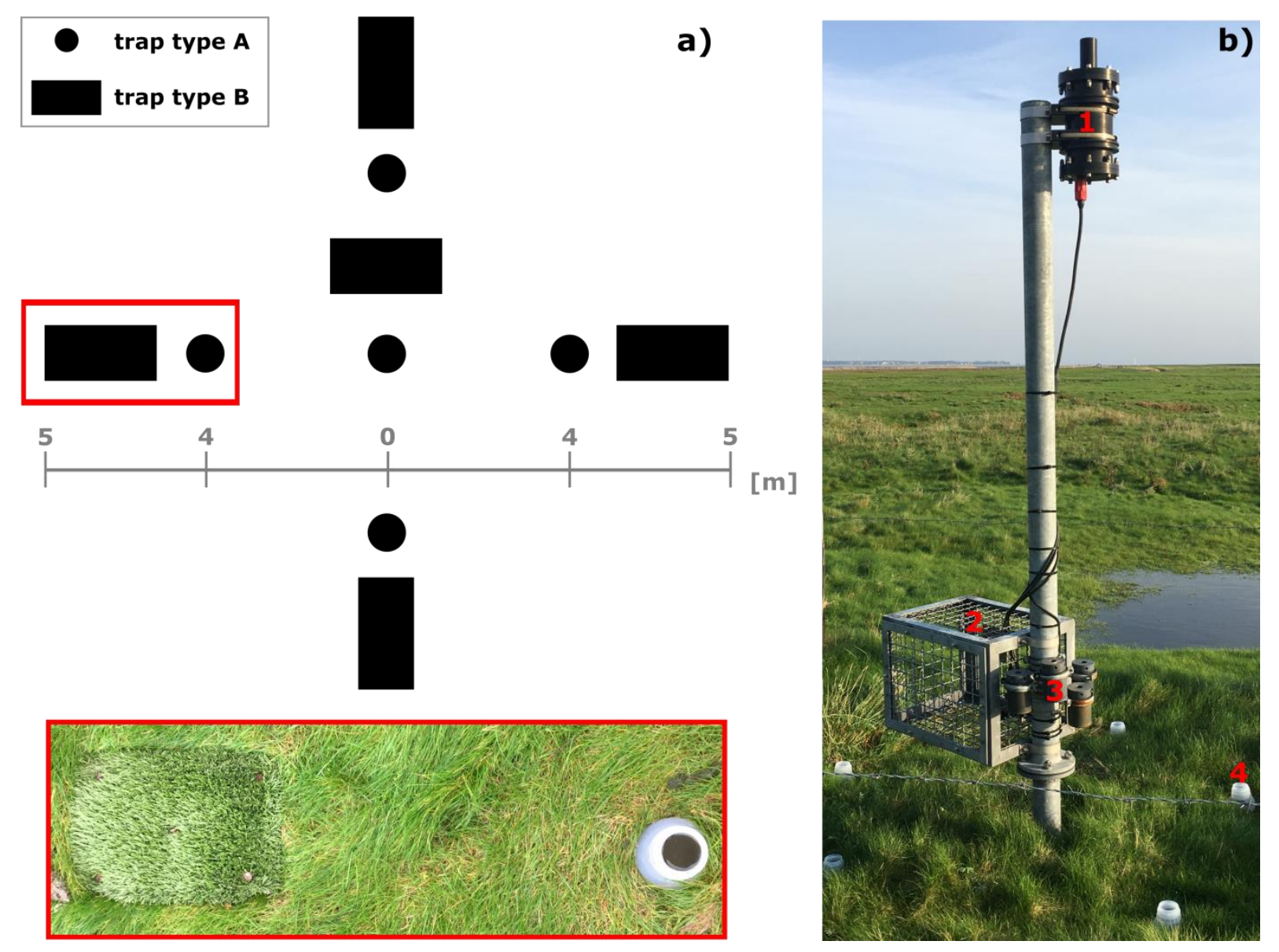

Figure 3-4: Sketch of a top view trap cluster, consisting of five bottles (trap type A) and five turf mats (trap type B) installed around the cluster center at a distance of approximately five meters. The red inset shows a photo of trap types $A$ and $B$ as installed in the field. Figure 3-4b shows a picture of the turbidity measurement station T-7 on the marshland of Langeness consisting of a pressure housing (1) in which data acquisition and transmission unit is located and the metal grid cage (2) in which the OBS sensor unit is mounted to protect it against flotsam. Next to the OBS sensor unit, 4 sediment accumulators SB (3) are mounted. The stations in the tidal flat have additional sediment accumulators ST in approximately 1.2 meter above the SB accumulators. This construction is surrounded by five sediment traps (4). A more detailed description of the functionality (data acquisition and transmission) of the turbidity measurement stations is given in Hache et al. (2019). 


\subsubsection{Inundation criterion}

Note that many tides were measured during the experimental period but only a few tides reached the inundation criterion required for a complete inundation of the entire Hallig, and hence were referred to as inundations. An inundation of the entire Hallig occurs when the water level reaches the mean height of the non-permeable revetments. These high water levels are usually caused by strong westerly winds which are superimposed on high astronomical tides. Above this threshold, only the dwelling-mounts stand above the water (Figure 3-2b). Langeness can be divided into its historical parts named Nordmarsch located in the west with a mean height of the non-permeable revetments of $2.27 \pm 0.2 \mathrm{~m}$ above MSL and Alt-Langeness in the east with $2.4 \pm 0.16 \mathrm{~m}$ above MSL, reflecting the difference in mean elevation of the Hallig surface from west to east of about $0.2 \mathrm{~m}$ (Schindler and Willim 2015). The water level in the west refers to the interior tide gauge Kirchhofswarf and in the eastern part to the interior tide gauge Kirchwarf (Figure 3-3).

The appearance of Hallig Langeness with its dykes, revetments and breakwater constructions is equivalent to a man-made anthropogenic environment (Schindler 2014). Without these anthropogenic structures, Langeness would have been flooded 85 times in the winter season 2018/19. In fact, only 5 inundations occurred. In general, the sampled inundation per year ranged from 3 to 6 inundations (Table 3-1) which is in line with the 3 to 7 inundations between 1961 and 2010 reported by Schindler and Willim (2015). Hence, the investigated number of inundations in this study is representative.

\subsubsection{Laboratory sample preparation}

The samples from the sediment accumulators and from the trap clusters were prepared to quantify total mass of solids $\left(M_{s}\right)$, as well as clastic $\left(M_{c}\right)$ and organic $\left(M_{0}\right)$ fractions to calculate vertical accretion rates.

The samples of the sediment accumulators and from the bottles (trap type A) were wet sieved with deionised water to filter out seagrass and shells. The brackish water was decanted after at least two days of particle settling. To remove the surplus brackish water and its dissolved salt, the samples were rinsed with deionised water in a centrifuge container and centrifuged for 5 minutes at $1400 \mathrm{rpm}$. The separation between the sample and the remaining brackish water resulted in negligible salt content. Then the samples were rinsed with deionised water in a glass bowl and stirred until a homogeneous suspension was generated for subsequent uniform particle settling within the bowl. The samples were oven dried at $105^{\circ} \mathrm{C}$ for $24 \mathrm{~h}$ to determine 
$M_{s}$ by weighing. To determine the percentage of $M_{0}$, we used the loss on ignition (LOI) method. An aliquot was taken from the total sample $\left(M_{s}\right)$ and weighed. The aliquot was dried at $425^{\circ} \mathrm{C}$ for $2 \mathrm{~h}$ and reweighed again.

The mats (trap type B) were dried at $70{ }^{\circ} \mathrm{C}$ for at least $24 \mathrm{~h}$ and weighed. Then the mats were cleaned with a high-pressure cleaner, dried again for $24 \mathrm{~h}$ and reweighed. The difference weight corresponds to the total accumulated amount of $M_{s}$ on the mats. The percentage of organic material is determined by the mean value of the five adjacent bottles of the trap cluster and transferred to the mats because no precise determination of the respective organic proportion is possible after the cleaning process. To calculate the vertical accretion rates according to Schindler et al. (2014b), we used the annually calculated average values of $M_{s}$ and $M_{o}$ from trap types $A$ and $B$.

\subsubsection{Trap cluster data}

With the trap clusters, three winter seasons were sampled, 2016/17, 2017/18 and 2018/19, each from October to March. The key parameters are displayed in Table 3-1.

Table 3-1: Vertical marshland accretion rates $\Delta \mathrm{H}$ of three sampled winter seasons displayed as three year average of all trap clusters as well as the minimum and maximum values $\Delta \mathrm{h}$. Number of sampled inundations and the mean inundation height based on data from the interior tide gauges Kirchhofswarf (KHW) and Kirchwarf (KW) in m above m.s.l (Figure 3-3).

\begin{tabular}{|c|c|c|c|c|c|}
\hline $\begin{array}{l}\text { Winter } \\
\text { season }\end{array}$ & $\begin{array}{c}\text { number of } \\
\text { sampled } \\
\text { inundations }\end{array}$ & $\Delta \mathrm{H}[\mathrm{mm} / \mathrm{a}]$ & $\begin{array}{c}\Delta \mathrm{h} \min / \max \\
{[\mathrm{mm} / \mathrm{a}]}\end{array}$ & $\begin{array}{l}\text { KHW mean } \\
\text { inundation } \\
\text { height [m } \\
\text { a.m.s.I.] }\end{array}$ & $\begin{array}{c}\text { KW mean } \\
\text { inundation } \\
\text { height [m } \\
\text { a.m.s.I.] }\end{array}$ \\
\hline $16 / 17$ & 6 & $1.9 \pm 1.9$ & $0.4 / 6.5$ & $2.9 \pm 0.41$ & $2.94 \pm 0.45$ \\
\hline $17 / 18$ & 3 & $0.9 \pm 0.5$ & $0.1 / 1.8$ & $2.5 \pm 0.21$ & $2.44 \pm 0.35$ \\
\hline $18 / 19$ & 5 & $1.0 \pm 0.9$ & $0.1 / 2.7$ & $2.46 \pm 0.2$ & $2.56 \pm 0.15$ \\
\hline all & 14 & $1.3 \pm 1.1$ & $0.1 / 6.5$ & $2.62 \pm 0.27$ & 2.65 \\
\hline
\end{tabular}

In the season 2016/17, the turbidity measurement network was not fully developed and therefore the trap clusters remained in the ground for the whole season in order to collect the $M_{s}$ accumulation from the total number of six inundations cumulatively. In the following seasons, the procedure was unchanged for traps of type $B$ but the traps of type $A$ were sampled 
after each inundation to determine the $M_{s}$ accumulation for each event. For this purpose, the content of the five bottles per trap cluster (Figure 3-4a) was merged in order to obtain a robust average for each cluster and to reduce laboratory time. Altogether we sampled three inundations in season $2017 / 18$ and five inundations in season $2018 / 19$ that met the inundation criterion. In season 2018/19 two more trap fields of type A were added close to the turbidity measurement stations on the marshland (T-7, T-8; Figure 3-4b) to better investigate the correlation between SPM and accumulated $\mathrm{M}_{\mathrm{s}}$.

\subsubsection{Turbidity time series}

Within the two winter turbidity measurement periods from October 1, 2017 to February 15, 2018 and from September 01, 2018 until March 01, 2019 the turbidity network covered 229 to 440 tides (Table 3-2). The number of covered tides depends on the elevation of the individual turbidity measurement station (Figure 3-3) and the tidal range. Within the measured time series, eight inundations of Hallig Langeness were recorded by the turbidity measurement stations on the tidal flats (T-1 to T-6) that met the inundation criterion. Each inundation was additionally analyzed separately with sediment trap clusters and sediment accumulators. On Langeness itself, six out of the eight inundations were also recorded by at least one of the stations on the marshland $(T-7, T-8)$. For three inundations a simultaneous measurement of both stations on the marshland was possible.

Table 3-2: Total number of recorded tides within the turbidity measurement network and the total number of recorded tides that lead to an inundation of the entire Hallig according to the inundation criterion (cf. section 2.4) within winter seasons from October 1, 2017 to February 15, and from September 1, 2018 to March 01, 2019. Missing inundation measurements at station T-3 and T-4 were due to technical issues.

\begin{tabular}{ccc}
\hline station & measuring duration [tides] & $\begin{array}{c}\text { number of recorded } \\
\text { inundations }\end{array}$ \\
T-1 & 440 & 8 \\
T-2 & 431 & 8 \\
T-3 & 381 & 7 \\
T-4 & 401 & 7 \\
T-5 & 260 & 8 \\
T-6 & 229 & 8 \\
T-7 & 5 & 5 \\
T-8 & 4 & 4 \\
\hline
\end{tabular}


In order to investigate SPM response to changing meteorological conditions, ten minutes averaged mean wind speeds and directions from weather station Strucklahnungshörn are used which were kindly provided from the Climate Data Center (CDC) of the German Meteorological Service (DWD). The weather station is located at $54.4965^{\circ} \mathrm{N}$ and $8.8069^{\circ} \mathrm{E}$, about $22 \mathrm{~km}$ southeast of the tide gauge Hilligenley. In addition, wave data was provided by the responsible authority for coastal protection, national parks and marine protection (LKN-SH), determined by a sea state buoy, which is located in the southern tidal channel Süderaue at about $2.5 \mathrm{~km}$ distance to the tide gauge Hilligenley.

\subsubsection{Grain size analysis}

Laser granulometry was applied to measure the grain size distribution of all trap type $A$ and sediment accumulator samples. An aliquot was extracted from the total sample $\left(M_{s}\right)$ and treated for 30 seconds in a supersonic bath to fully disaggregate the fine-grained material. The measurements were carried out with a laser particle sizer LS13320 by Beckman\&Coulter. Each sample was measured three times in the range $0.4-2000 \mu \mathrm{m}$ for one minute and averaged. The Fraunhofer model was applied to calculate the grain size distribution from the particle sizer data.

\subsection{Results}

\subsubsection{Tidal flat SPM}

The North Frisian tidal flats are characterized by strong winds from westerly directions. Around Langeness, SPM values have a variable response to wind and tidal forcing, both spatially and temporally (detailed in Hache et al. 2019). Figure 3-5 exemplifies the correlation between averaged SPM and wind speed from two wind direction quadrants on tidal scales for various stations. The SPM depends, among other things, on spatial factors such as (i) areas with shallower water are more reactive to wind speed and wind induced waves and, consequently, reach higher SPM concentrations (e.g. T-1 in Figure 3-5), (ii) areas adjacent to a tidal channel (T4) are constantly higher in SPM concentration at lower wind speed due to higher tidal current velocities, (iii) areas with distinct constrictions between spur dykes (Figure 3-2d) can only benefit from a narrow range of wind directions approaching the Hallig roughly orthogonal, i.e. parallel to the spur dykes. Therefore, in case of station T-2, the best correlation between SPM 
and wind speed was not achieved within the main wind direction quadrants (S-W, W-N) but instead came from the East to South wind direction quadrant with an adjusted coefficient of determination of 0.48 . However, such wind directions are not typical during inundations and were not recorded in 2017 to 2019 (Table 3-3), so this result will not be discussed further. In general, a strong wind from the S-W quadrant generates the highest overall SPM concentrations throughout the entire turbidity measurement network on the tidal flat.
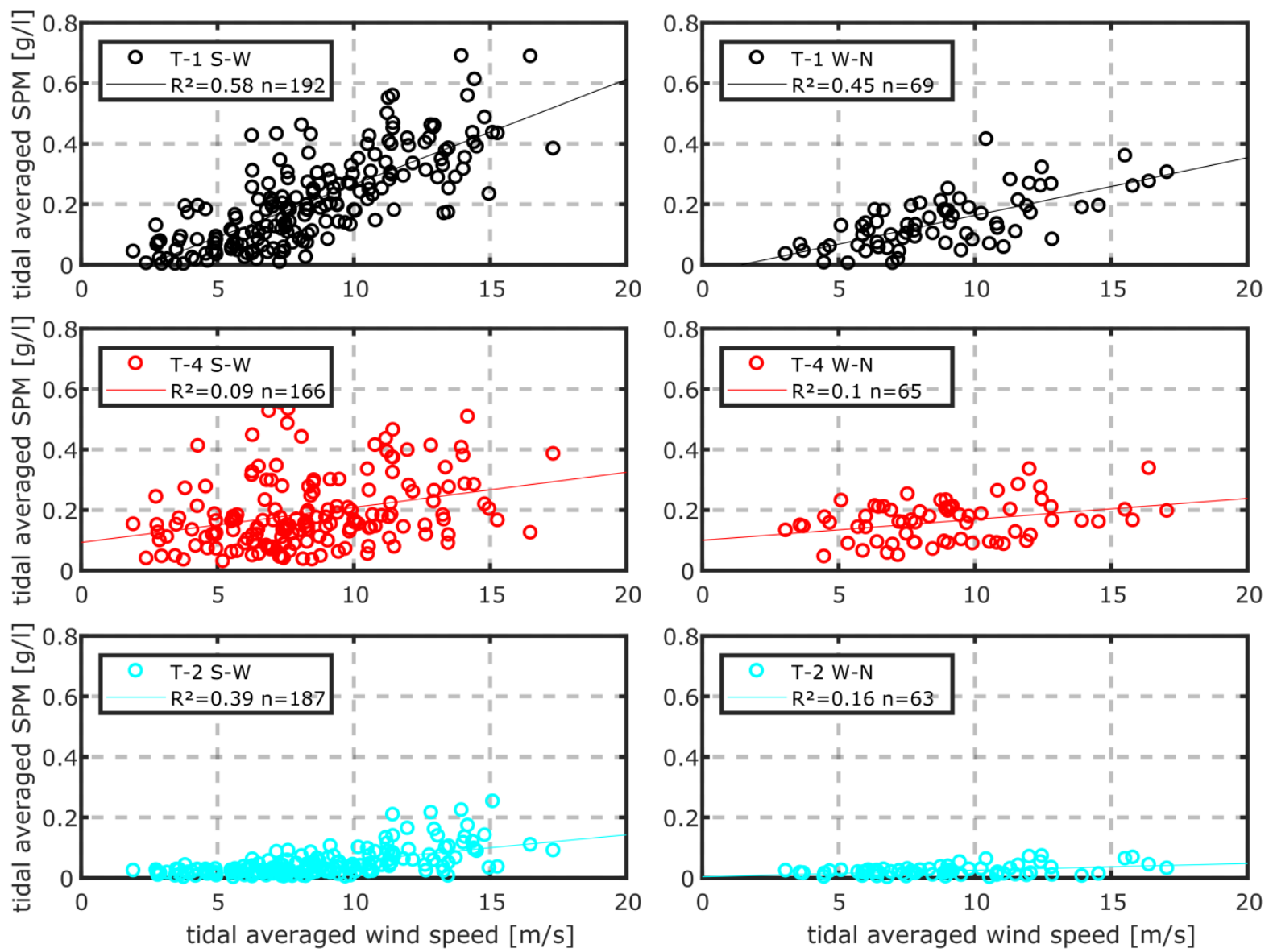

Figure 3-5: Linear regression analysis between tidal averaged wind speed and SPM concentrations at three different sites from the turbidity measurement stations T-1, T-2 and T-4 (Figure 3-3), separated for the two main wind direction quadrants from south to west (left) and west to north (right), during both measured winter periods from October 1, 2017 to February 15, 2018 and from September 1, 2018 to March $01,2019 . R^{2}$ is the adjusted coefficient of determination after Pearson and $\mathrm{n}$ is the number of observations. The $p$-values are below the assumed significance level of 0.05 .

Wind, wave action, and tidal currents are the main factors controlling SPM concentration during inundations. Strong variations in SPM throughout the whole turbidity measurement network in the tidal flat are shown in Table 3-3. 
Table 3-3: Tidal averaged parameters for eight different inundations measured at Langeness. Ten minutes averaged mean wind speeds and directions from the weather station

Strucklahnungshörn (located $22 \mathrm{~km}$ southeast of the tide gauge Hilligenley) and ten minutes averaged mean significant wave heights from the sea state buoy Süderaue. Mean SPM values from the whole turbidity network in the tidal flat (av SPM) and mean $\mathrm{M}_{\mathrm{s}}$ accumulation rates in the trap clusters and mean inundation heights above marsh surface from both interior tide gauges Kirchhofswarf and Kirchwarf.

\begin{tabular}{|c|c|c|c|c|c|c|}
\hline Inundation & $\begin{array}{l}\text { wind } \\
\text { speed } \\
{[\mathrm{m} / \mathrm{s}]}\end{array}$ & $\begin{array}{c}\text { wind } \\
\text { direction } \\
\text { [degree] }\end{array}$ & $\begin{array}{l}\text { significant } \\
\text { wave } \\
\text { height [m] }\end{array}$ & $\begin{array}{c}\text { av } \\
\text { SPM } \\
{[\mathrm{g} / \mathrm{l}]}\end{array}$ & $\begin{array}{c}\text { mean } \mathrm{M}_{\mathrm{s}} \\
\text { accumulation } \\
{\left[\mathrm{g} / \mathrm{m}^{2}\right]}\end{array}$ & $\begin{array}{c}\text { mean } \\
\text { inundation } \\
\text { height }[\mathrm{m}]\end{array}$ \\
\hline $\begin{array}{l}1 . \\
(08.12 .2017)\end{array}$ & $\begin{array}{l}8.1 \pm \\
2.6\end{array}$ & $268.9 \pm 19.8$ & $0.41 \pm 0.11$ & $\begin{array}{l}0.19 \pm \\
0.12\end{array}$ & $132.8 \pm 90$ & $0.93 \pm 0.13$ \\
\hline $\begin{array}{l}2 . \\
(04.01 .2018)\end{array}$ & $\begin{array}{l}10.7 \pm \\
3.8\end{array}$ & $280.6 \pm 8.7$ & $0.48 \pm 0.19$ & $\begin{array}{l}0.28 \pm \\
0.17\end{array}$ & $158.6 \pm 84.4$ & $1.12 \pm 0.11$ \\
\hline $\begin{array}{l}3 . \\
(12.02 .2018)\end{array}$ & $\begin{array}{l}14.2 \pm \\
1.7\end{array}$ & $243.3 \pm 11$ & $0.69 \pm 0.11$ & $\begin{array}{l}0.39 \pm \\
0.15\end{array}$ & $179.6 \pm 118.5$ & $1.08 \pm 0.13$ \\
\hline $\begin{array}{l}4 . \\
(08.12 .2018)\end{array}$ & $\begin{array}{l}14.6 \pm \\
1.5\end{array}$ & $239.5 \pm 12.5$ & $0.72 \pm 0.13$ & $\begin{array}{l}0.19 \pm \\
0.13\end{array}$ & $100.6 \pm 71.7$ & $0.96 \pm 0.1$ \\
\hline $\begin{array}{l}5 . \\
(08.01 .2019)\end{array}$ & $\begin{array}{l}14.5 \pm \\
2.3\end{array}$ & $332.1 \pm 12.9$ & $0.62 \pm 0.11$ & $\begin{array}{l}0.16 \pm \\
0.1\end{array}$ & $99.2 \pm 50.2$ & $1.26 \pm 0.08$ \\
\hline $\begin{array}{l}6 . \\
(09.02 .2019)\end{array}$ & $\begin{array}{l}17.3 \pm \\
1.4\end{array}$ & $227.1 \pm 7$ & $0.74 \pm 0.14$ & $\begin{array}{l}0.32 \pm \\
0.18\end{array}$ & $224.8 \pm 206.5$ & $0.78 \pm 0.02$ \\
\hline $\begin{array}{l}7 . \\
(05.03 .2019)\end{array}$ & $\begin{array}{l}15.4 \pm \\
2.8\end{array}$ & $251.6 \pm 3.7$ & $0.77 \pm 0.14$ & $\begin{array}{l}0.23 \pm \\
0.18\end{array}$ & $172.8 \pm 167.5$ & $0.99 \pm 0.1$ \\
\hline $\begin{array}{l}8 . \\
(10.03 .2019)\end{array}$ & $\begin{array}{l}11.4 \pm \\
10.8\end{array}$ & $256 \pm 3.6$ & $0.54 \pm 0.25$ & $\begin{array}{l}0.13 \pm \\
0.09\end{array}$ & $79.9 \pm 58.5$ & $0.96 \pm 0.07$ \\
\hline
\end{tabular}

Figure 3-6 shows the development of prevailing wind conditions over four preceding tides and the subsequent inundation. An increasing wind speed over several tides, with south to west directions (Figure 3-6a), produces the highest SPM concentration at inundation number 6 (Figure 3-6b), both on average and at each individual station. Note the small variability in wind speed and direction within each tide compared to inundation number 5 (Figure 3-6c) and especially inundation number 8 (Figure 3-6e). A stronger variation in wind speed and wind 
direction that in addition tend towards northerly directions (inundation number 5) cause overall lower concentrations and SPM decrease by $50 \%$ between the last two tides (Figure 3-6d). The fourth tide (see Figure 3-6c, d) follows an often recognizable pattern within our longterm measurements. High SPM concentrations are triggered by high wind speeds following relatively calm periods (tide 1 to 3 ) implying that tides that lead to an inundation do not necessarily have the highest overall SPM concentrations. In comparison to inundation number 6, significant variability in wind conditions within a tide does not lead to an increase in SPM at inundation number 8 which is highlighted by the large error bars in Figure 3-6e and the overall low average SPM concentrations (Figure 3-6f).
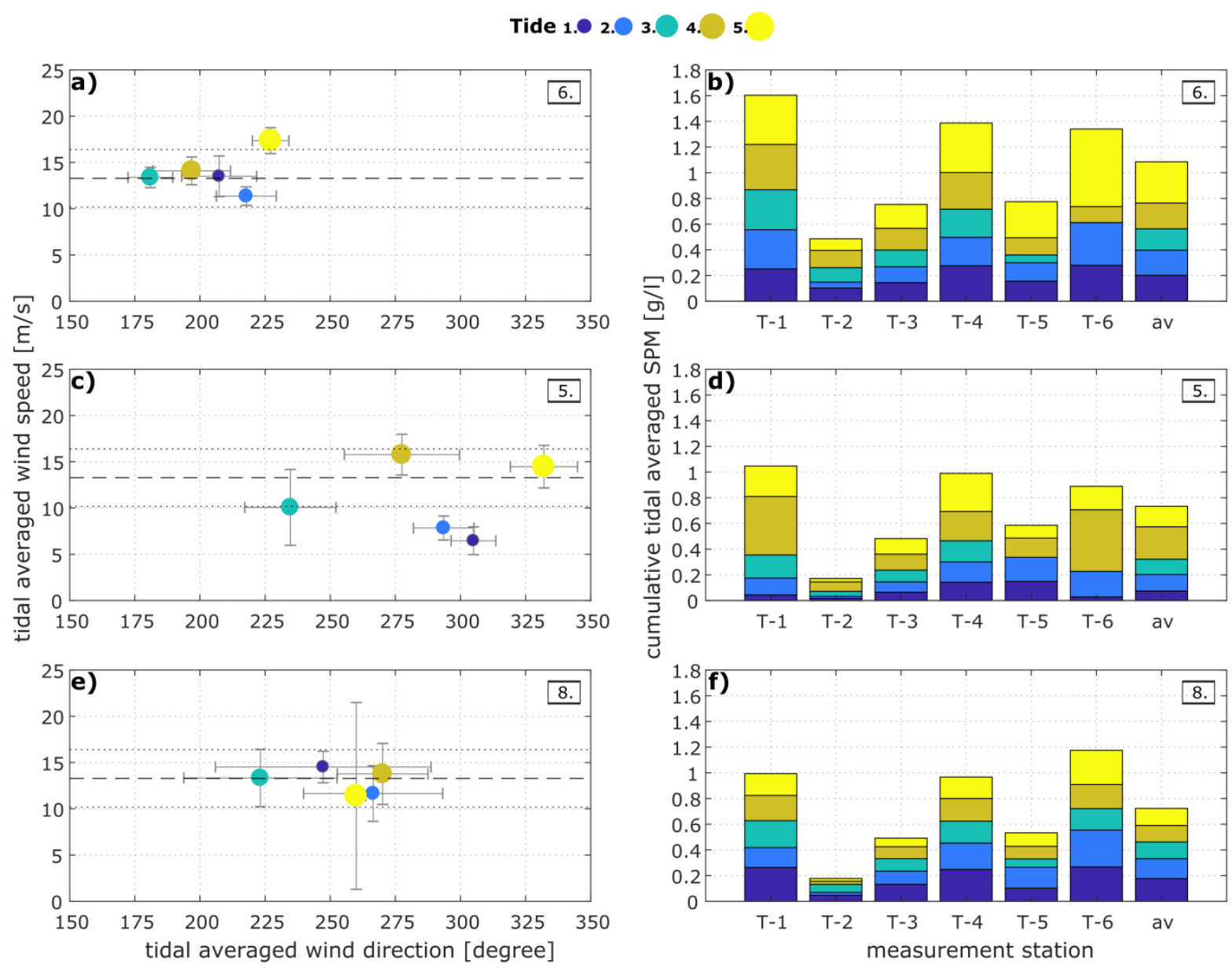

Figure 3-6: Left (a, c, e); the tidal averaged development of wind speed and wind direction of five tides that end up in the inundations number 6, 5 and 8 within the fifth tide (yellow). The error bars show the standard deviations which indicate tidal averaged wind condition variability. The dashed line shows the average wind speed of $13.3 \mathrm{~m} / \mathrm{s}$ from all 8 inundation cycles and their standard deviation of $3 \mathrm{~m} / \mathrm{s}$ (dotted lines). Right ( $b, d, f)$; the associated tidal averaged SPM for stations in the tidal flat and the average of all stations per tide (av). Inundation number 6 shows the highest average SPM concentration, followed by inundation number 5 and inundation number 8 with the lowest average SPM concentration. Missing tidal averaged SPM at station T-5 and/or T-6 are due to too low water levels during some tides. 


\subsubsection{SPM availability}

The inundation-induced $M_{s}$ accumulation values on Langeness vs. SPM on the tidal flats are displayed in Figure 3-7. Values during eight inundations indicate that higher SPM concentrations on the tidal flat result in an increased $M_{s}$ accumulation on the marshland. In order to investigate the quantities of available SPM more specifically, we directly compare the SPM concentrations of two stations on the tidal flat (T-4 and T-1) with the nearby stations on the marshland (T-7 and T-8; Figure 3-8).

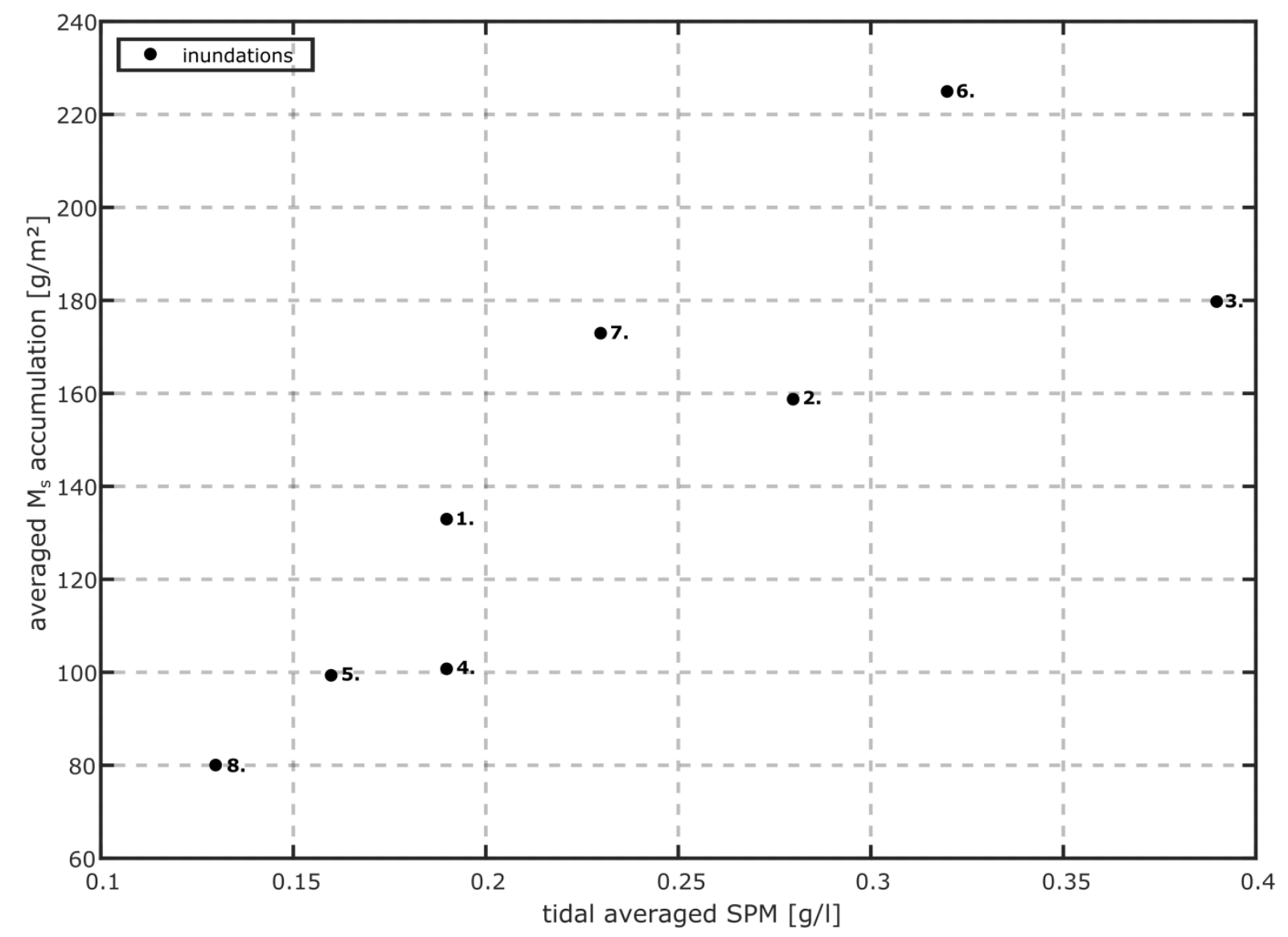

Figure 3-7: Overall tidal averaged SPM of the measurement stations in the tidal flat (T-1 to T-6) and averaged Ms accumulation from trap clusters on the marshland for eight sampled inundations in 2017/18 and 2018/19 (Table 3-3).

The comparison shows a large SPM difference between the neighboring station pairs that indicates a large contrast between the available SPM on the tidal flat and the SPM on the marshland. In other words, a significant amount of SPM does not reach the marshland of Langeness. An increase of the SPM availability on the tidal flat causes only a minor increase in the marshland SPM (Figure 3-8). The average SPM availability is $0.23 \mathrm{~g} / \mathrm{l}$ in the west and $0.29 \mathrm{~g} / \mathrm{I}$ 
in the east of Langeness. In addition, the exploitation of the potential SPM availability is on average $20 \%$ or $0.06 \mathrm{~g} / \mathrm{l}$ in the east, and as low as $9 \%$ in the west or $0.02 \mathrm{~g} / \mathrm{l}$.

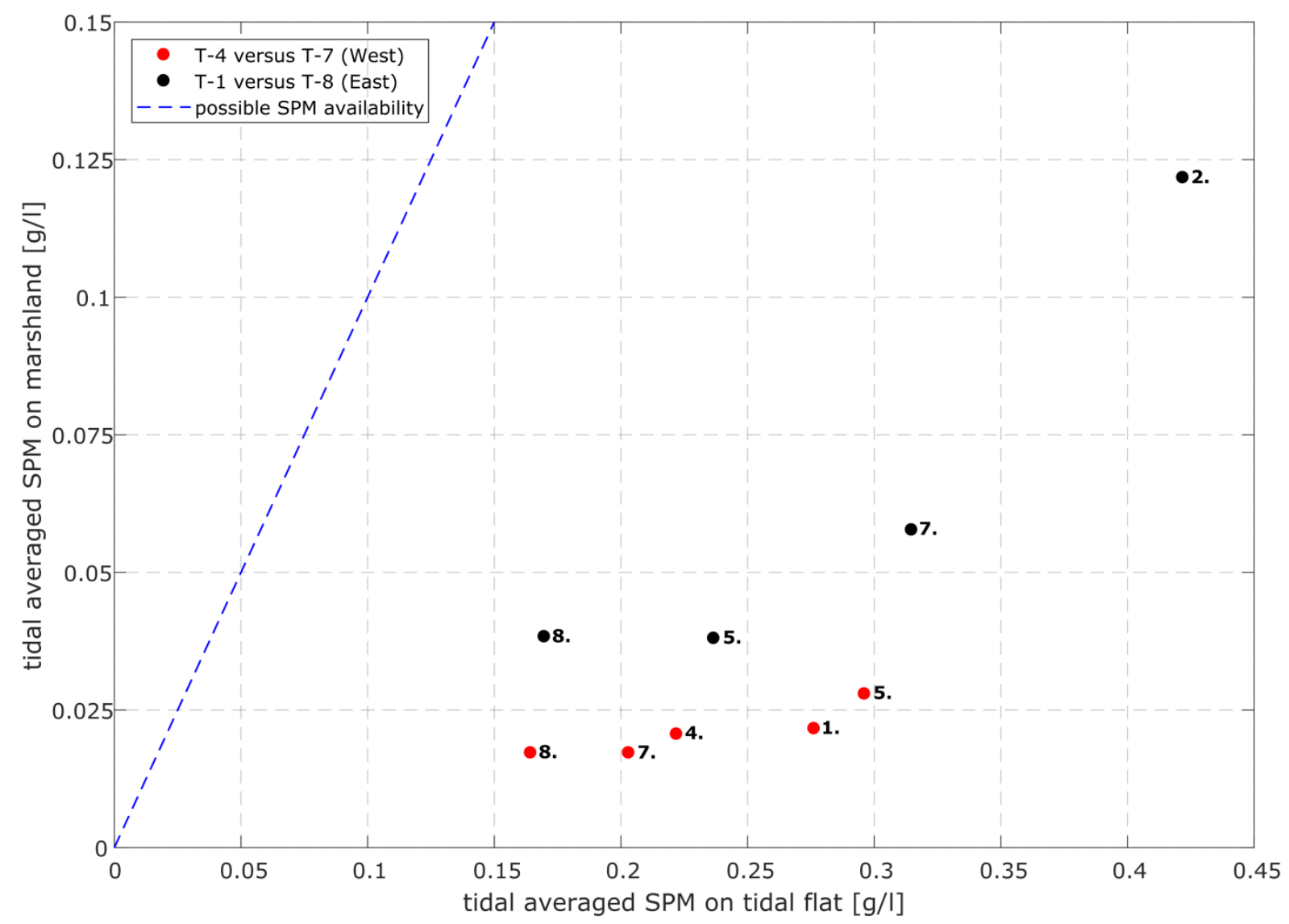

Figure 3-8: Comparison of the determined tidal averaged SPM on the tidal flat (T-4, T-1) to the respective marshland area $(T-7, T-8)$ for the six individual inundations where water level was high enough to activate at least one of the marshland stations (Table 3-2 and Table 3-3). The ascertained SPM concentrations at station T-4 are compared with the SPM concentrations at station T-7 (red dots) and T-1 with T-8 (black dots), respectively. The number besides the individual dots indicate the number of the inundation. The blue dashed line indicates the highest possible SPM on the marshland based on the SPM availability on the tidal flat.

To assign the measured SPM in $\mathrm{g} / \mathrm{I}$ on the marshland to the sediment accumulation in $\mathrm{g} / \mathrm{m}^{2}$, two additional trap type A fields were installed next to the turbidity stations T-7 and T-8 in the winter season $2018 / 19$. The results indicate a strong correlation between the marshland SPM and the nearby accumulated $\mathrm{M}_{\mathrm{s}}$ (Figure 3-9). 


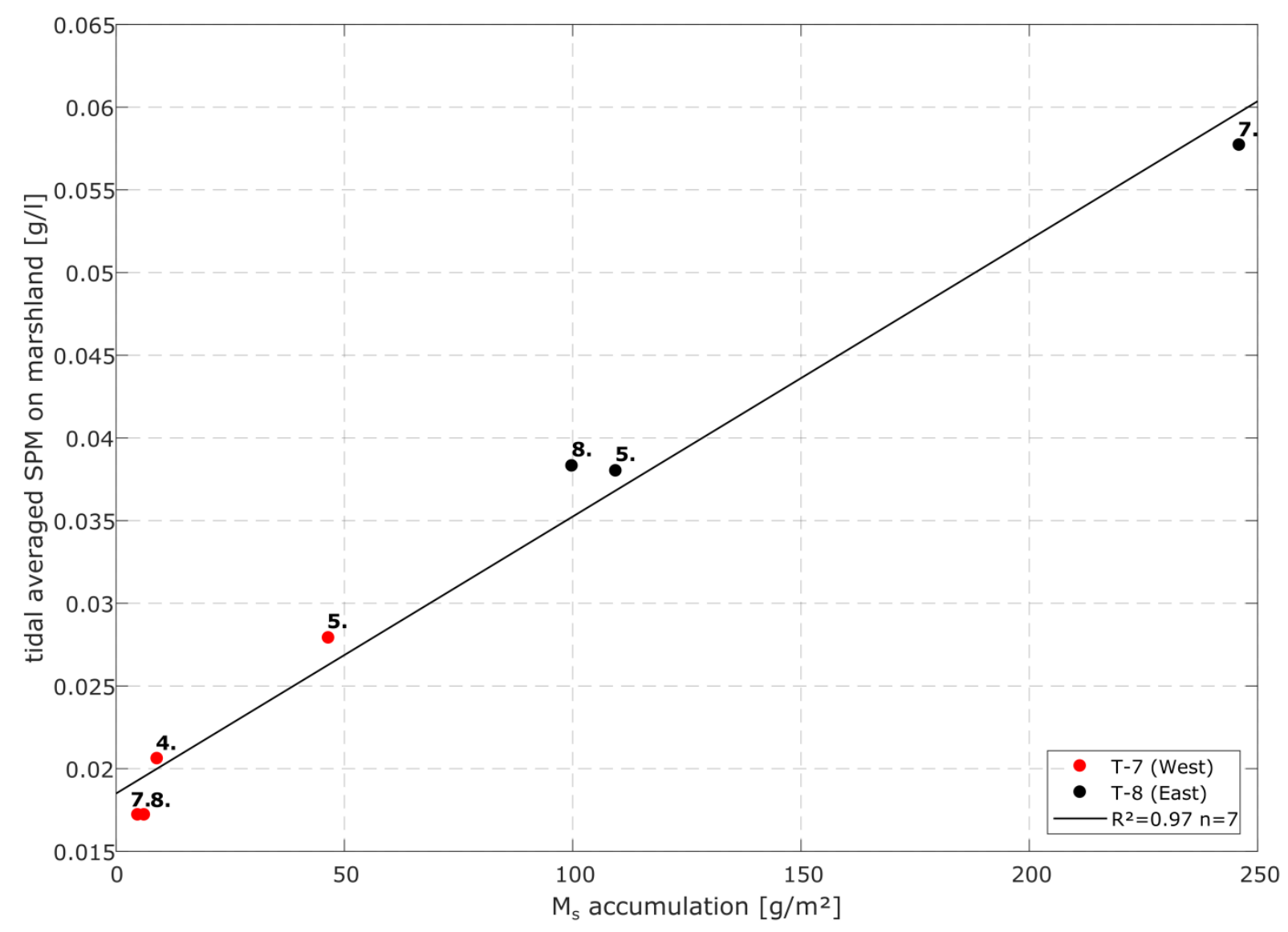

Figure 3-9: Linear regression analysis between averaged SPM at the two stations on the marshland (T-7 and $\mathrm{T}-8)$ and Ms accumulation of traps type $A$ next to both stations for the inundations during the winter season 2018/19 where water level was high enough to activate the respective station. The numbers indicate the number of the inundation (Table 3-3). The regression equation is $y=1.67 *$ $10^{-4} * M_{S}+0.0185 . \mathrm{R}^{2}$ is the adjusted coefficient of determination after Pearson and $\mathrm{n}$ is the number of observations.

The average accretion rate in winter $18 / 19$ of $1.0 \mathrm{~mm}$ (Table $3-1$ ) shows that the $M_{s}$ accumulation per inundation has to be on average five times higher to compensate for the recent $\mathrm{MHW}$ increase of $5 \mathrm{~mm} / \mathrm{a}$ (Schindler et al. 2014a). Based on five times the average $\mathrm{M}_{\mathrm{s}}$ accumulation in winter $18 / 19$ at the trapping fields next to stations $\mathrm{T}-7$ and $\mathrm{T}-8, \mathrm{M}_{\mathrm{s}}$ accumulations of $83.3 \mathrm{~g} / \mathrm{m}^{2}$ and $759 \mathrm{~g} / \mathrm{m}^{2}$, respectively, would be necessary to keep pace with MHW increase. Using the linear regression in Figure 3-9, these values translate into SPM values of $0.03 \mathrm{~g} / \mathrm{l}$ at station $\mathrm{T}-7$ and $0.15 \mathrm{~g} / \mathrm{I}$ at station T-8. As a conservative estimate, the SPM on the tidal flat has to exceed a threshold of at least $0.15 \mathrm{~g} / \mathrm{l}$ in order to ensure $M_{s}$ accumulation quantities that keep pace with the rising MHW. Note that no transport limitations may hinder the SPM to get onto the marshland in this scenario. The suggested threshold is exceeded for all inundations at stations T-4 and T-1 on the tidal flat, which are related to the related marshland stations T-7 and T-8, respectively (Figure 3-8). 


\subsubsection{SPM transport}

In general, the average $M_{s}$ accumulation on the marshland increases with increasing SPM availability on the tidal flats (Figure 3-7). On a smaller spatial scale there are clear differences in $M_{s}$ accumulation. The effect of coastal protection measures on today's $M_{s}$ accumulation was investigated with six trap clusters at three locations on the marshland near the turbidity measurement stations T-1, T-2 and T-3 (see section 2.3 and Figure 3-3). For this investigation, only trap type A data from the trap clusters were used due to the consistent sampling after each inundation. Two trap clusters were installed at each location, differing in the barrier height of the directly related protection measure, while the distance to the Hallig margin has been tried to keep almost constant for the individual pairs (Table 3-4).

Table 3-4: Key parameters of all trap cluster pairs with different barrier heights ( $\mathrm{H}=\mathrm{high}$ and $\mathrm{L}=\mathrm{low}$ ). The barrier heights are measured from the seabed of the nearby turbidity measurement station to the top of the barrier (elevation data from GNSS topographical survey of the LKN.SH). The average $M_{s}$ accumulation is the mean of all eight inundations. The average inundation SPM (SPM availability on the tidal flat) is the mean of all tidal averaged SPM of eight inundations measured at the nearby turbidity measurement station on the tidal flat ( $\mathrm{T}-1$ for $1 \mathrm{H}$ and $1 \mathrm{~L}, \mathrm{~T}-2$ for $2 \mathrm{H}$ and $2 \mathrm{~L}, \mathrm{~T}-3$ for $3 \mathrm{H}$ and $3 \mathrm{~L}$ ).

\begin{tabular}{ccccc}
\hline trap cluster & $\begin{array}{c}\text { barrier height } \\
{[\mathrm{m}]}\end{array}$ & $\begin{array}{c}\text { distance to Hallig } \\
\text { margin }[\mathrm{m}]\end{array}$ & $\begin{array}{c}\text { average } \mathbf{M}_{\mathbf{s}} \\
\text { accumulation } \\
{\left[\mathrm{g} / \mathrm{m}^{2}\right]}\end{array}$ & $\begin{array}{c}\text { average } \\
\text { inundation SPM } \\
{[\mathrm{g} / \mathrm{I}]}\end{array}$ \\
\hline $\mathbf{1 H}$ & $2.69 \pm 0.14$ & 30 & $263 \pm 132$ & $0.34 \pm 0.12$ \\
$\mathbf{1 L}$ & $2.22 \pm 0.04$ & 40 & $329 \pm 151$ & $0.34 \pm 0.12$ \\
$\mathbf{2 H}$ & $3.58 \pm 0.23$ & 32 & $75 \pm 32$ & $0.06 \pm 0.05$ \\
$\mathbf{2 L}$ & $3.2 \pm 0.11$ & 33 & $149 \pm 49$ & $0.06 \pm 0.05$ \\
$\mathbf{3 H}$ & $3.24 \pm 0.06$ & 120 & $43 \pm 21$ & $0.14 \pm 0.07$ \\
$\mathbf{3 L}$ & $2.87 \pm 0.11$ & 121 & $176 \pm 46$ & $0.14 \pm 0.07$ \\
\hline
\end{tabular}

Location 1 has the lowest barrier heights and the highest SPM availability of all three locations and shows the smallest difference in the $M_{s}$ accumulation between trap cluster $1 \mathrm{H}$ (high barrier) and $1 \mathrm{~L}$ (low barrier). Nevertheless, on average $25 \%$ more $\mathrm{M}_{\mathrm{s}}$ can be found in trap cluster $1 \mathrm{~L}$ compared to trap cluster $1 \mathrm{H}$ (Table 3-4). Beyond high variability between individual inundations, the trap clusters at Location 1 yield constantly highest values for all inundations (compared to the other two locations) as well as constantly higher values behind the lower barrier (1L vs. $1 \mathrm{H}$; Figure 3-10). At Location 2, barrier heights are highest, above 3 meters due to 
the deeper tidal flat bathymetry (Figure 3-3) and this locality shows the lowest SPM availability and generally low $M_{s}$ accumulation. However, the $M_{s}$ accumulation is on average twice as high in the lower barrier trap cluster $2 \mathrm{~L}$ than in the adjacent higher barrier trap cluster $2 \mathrm{H}$. Barrier height as well as SPM availability at Location 3 is intermediate compared to the other two locations (Table 3-4). The distance to the Hallig margin is approximately three times farther compared to the other locations. The difference in $\mathrm{M}_{\mathrm{s}}$ accumulation between trap cluster $3 \mathrm{H}$ and $3 \mathrm{~L}$ is highest, by about a factor of 4 . The data shows that throughout all stations and all measured inundations, $M_{s}$ accumulation is constantly higher behind the lower barriers (Figure 3-10).

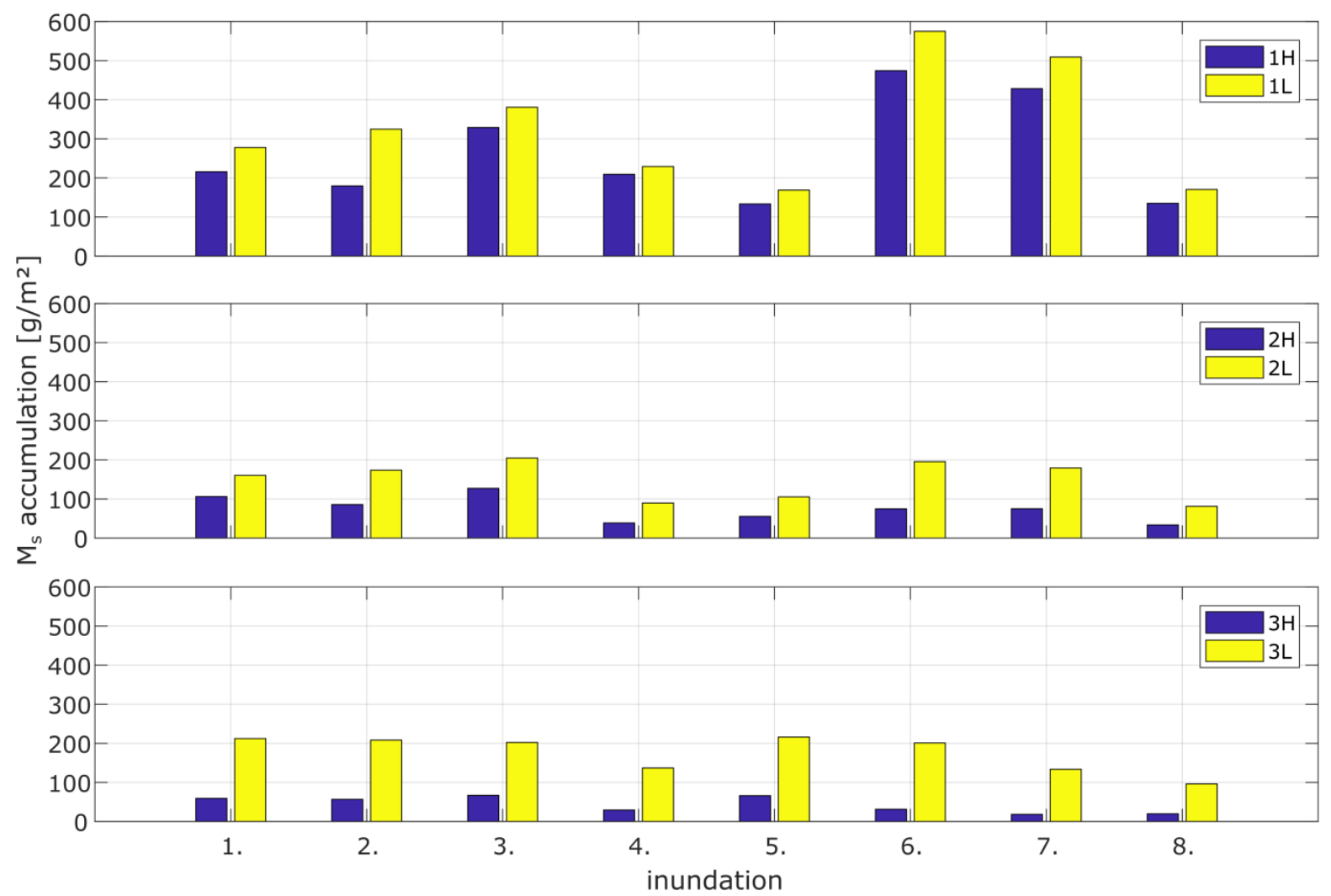

Figure 3-10: Ms accumulation $\left[\mathrm{g} / \mathrm{m}^{2}\right]$ for all eight inundations (Table 3-3) at three locations (1, 2 and 3 related to stations T-1, T-2 and T-3; Figure 3-3) with two trap clusters each related to different barrier heights ( $\mathrm{H}=$ high, $\mathrm{L}=$ low, Table 3-4).

\subsubsection{Grain size}

To investigate the effect of the coastal revetment on the grain size distribution grain size analyses are compared between the sediment accumulators and adjacent trap clusters near the Hallig margin at the wind and wave exposed area at station T-1 and in a low energetic area at station T-2. 
Among all inundations investigated, inundation 3 shows the highest SPM availability in the tidal flat around Langeness while inundation 6 shows the highest averaged $M_{s}$ accumulation (Figure 3-7). The grain size distribution of the sediment accumulators SB (Base) and ST (Top) at the wind and wave exposed station T-1 reveals a good mixing within the water column (Figure 3-11). The SPM in the tidal flat was coarser-grained for inundation 3 compared to inundation 6. The nearby trap clusters $(1 \mathrm{H}, 1 \mathrm{~L})$ show that the coarsest grain size class $(63-200 \mu \mathrm{m}$, very fine to fine sand) is more frequent in the trap cluster behind the lower barrier (1L) compared to the high barrier $(1 \mathrm{H})$ for both inundations. Clearly, the sand fraction is more hampered in reaching the marshland due to the higher barrier (Figure 3-11). The coarsest grain size class (63-200 $\mu \mathrm{m})$, however, always makes up the majority of the available sediments with a median grain size of $83 \pm 16 \mu \mathrm{m}$ at station T-1 within the sediment accumulator SB and $67 \pm 26 \mu \mathrm{m}$ in the sediment accumulator ST. Fine sand can account for up to $70 \%$ within the sediment accumulators after an inundation and is predominantly present at higher energetic areas due to wind and wave or tidal forcing at stations T-1, T-3 and T-4. This prevailing grain size fraction fails to overcome the coastal revetment because a median grain size of $39 \pm 10 \mu \mathrm{m}$ for all eight inundations was measured at the trap clusters $(1 \mathrm{H}, 1 \mathrm{~L})$ and $32 \pm 11 \mu \mathrm{m}$ as average of all trap clusters on the marshland.
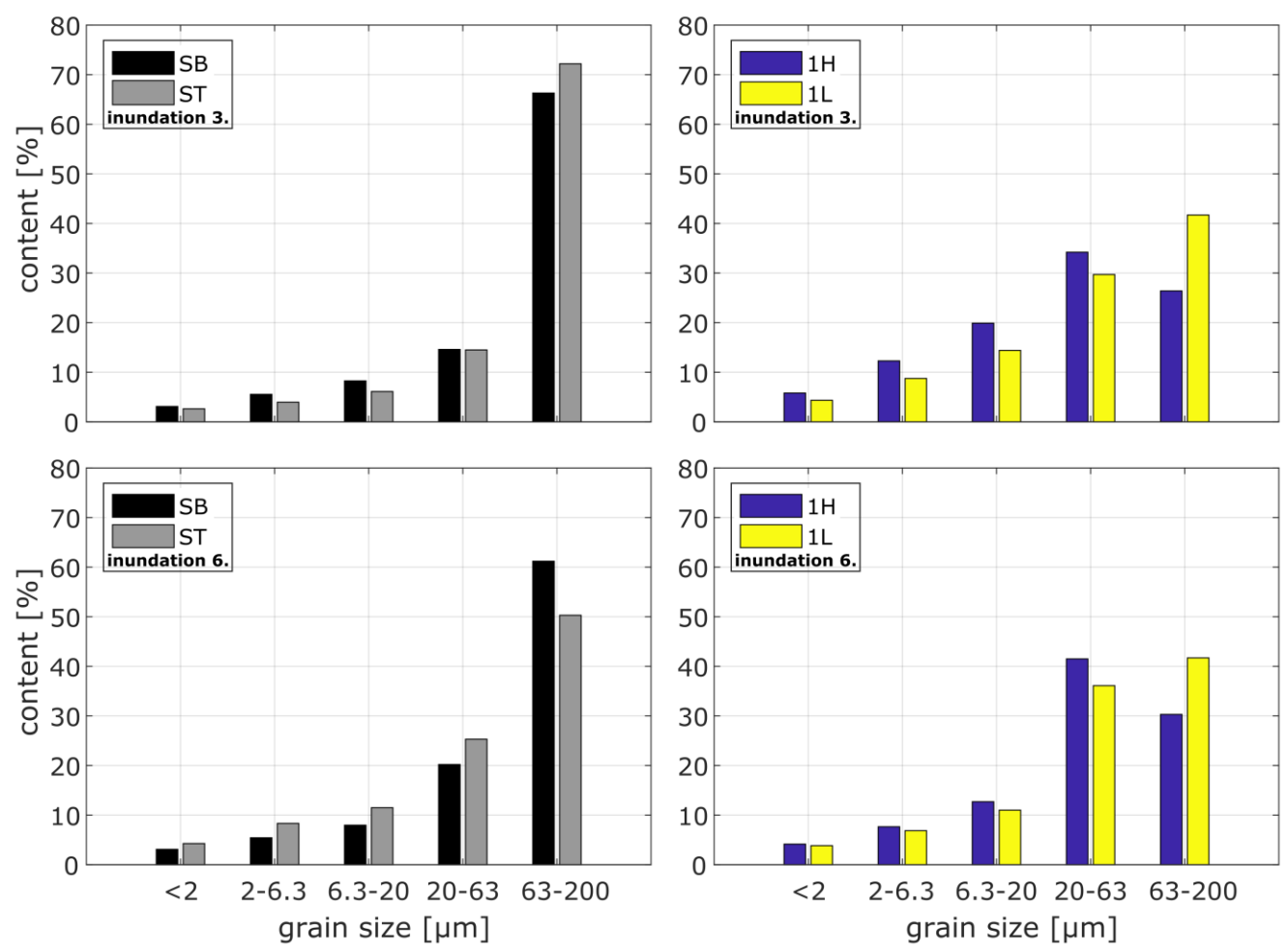

Figure 3-11: Grain size analysis of samples from the sediment accumulators SB (Base) and ST (Top) at station $\mathrm{T}-1$ and the adjacent trap clusters $1 \mathrm{H}$ and $1 \mathrm{~L}$, for the two inundations number 3 and number 6 (Table 3-3). 
The largest deviation from the particle size distribution within the sediment accumulator at higher energetic areas is found in the low energetic area at station T-2 (Figure 3-12). The small scatter of the median grain sizes at station T-2 underlines the calming effect of the adjacent spur dykes even at harsh weather conditions during inundation. Therefore, the very fine to fine sand only accounts for approximately $25 \%$ resulting in median grain sizes of $30 \pm 2 \mu \mathrm{m}$ in SB and $34 \pm 7 \mu \mathrm{m}$ in the ST accumulator. The grain sizes at station T-2 are almost the same as in the adjacent trap clusters which is indicated by the close positioning of the median grain size values to the 1:1 line in Figure 3-12. The close positioning of the median grain size values to the 1:1 line only applies at station T-1 for inundation number 4, i.e. the first inundation that occurred in the season 2018/19 where we found the lowest particle size distribution of the measured inundations in the tidal flat.
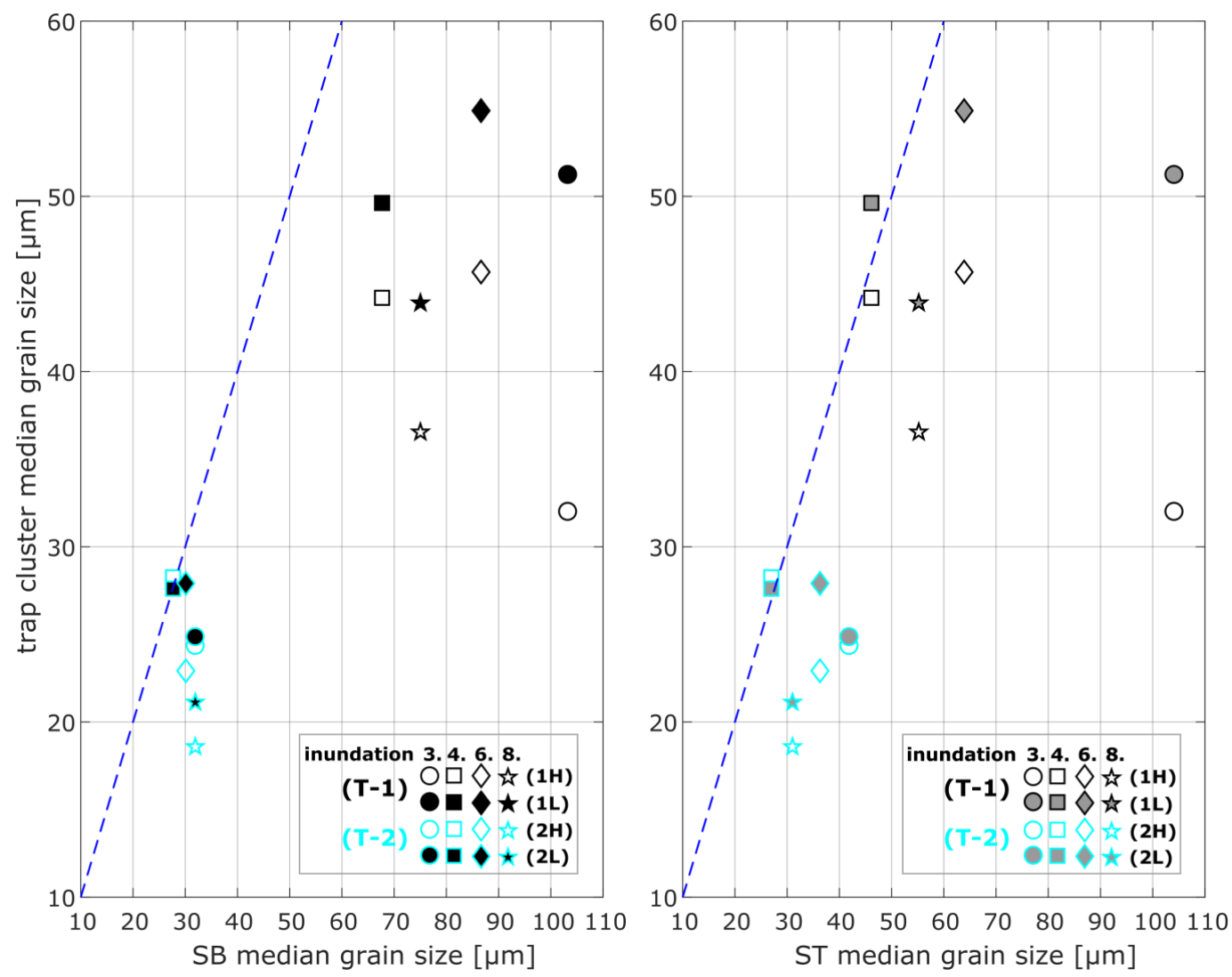

Figure 3-12: Median grain size comparison for four inundations number 3, 4, 6, and 8 (3 - circles, 4 squares, 6 - diamonds, 8 - stars) between the sediment accumulators at tidal flat stations T-1 (black) and $\mathrm{T}-2$ (cyan), and trap cluster samples from clusters $1 \mathrm{H}, 2 \mathrm{H}$ (unfilled) and $1 \mathrm{~L}, 2 \mathrm{~L}$ (filled). The blue dashed line indicates the 1:1 ratio of median grain sizes in the trap clusters versus sediment accumulator SB (Base, left) or ST (Top, right). For this grain size comparison, only sediment accumulator sample results are shown where it was possible to empty the accumulators immediately before the inundation and sample it as soon as tidal flats were accessible again after the inundation (maximum two days) to minimize potential bias in the sediment accumulators caused by subsequent tides. 


\subsection{Discussion}

The simple regression model of wind speeds, subdivided into the two most relevant westerly wind direction quadrants underlines that meteorological forcing strongly affects SPM concentrations in the study area (Figure 3-5). This is consistent with other studies (Fettweis et al. 2006; Fettweis et al. 2007; Fettweis et al. 2012; Jorge and van Beusekom 1995). A simple linear regression model shows a strong relationship between wind speed and SPM. Multiple linear regression, including the significant wave height, shows no significantly better relationships, likely due to the fact that the wave buoy is located approximately $2.5 \mathrm{~km}$ off the site. SPM variations in deeper regions near tidal channels are controlled by tidal currents (e.g. Ricklefs 1998; Albers and Lieberman 2007). However in the shallow tidal flats around Langeness, the tidal effect is largely outcompeted by strong winds that drive higher SPM concentrations (Figure 3-5). We therefore attempted a correlation over the tidal range because correspondingly higher current velocities may also cause increase in SPM (e.g. Reuter et al. 2009; Bartholomä et al. 2009). This attempt, however, led to poor correlation results, comparable to results reported by Fettweis et al. (2010), who showed the relation between tidal range and tidal average SPM concentration which are overprinted by high wave conditions and wind-induced advection. We therefore also suggest that flow-induced SPM increase is strongly superimposed by the impact of wind and wave action. Only higher SPM values at lower wind speed indicates the influence of tidal currents. Investigations in the middle of a tidal channel between the islands of Langeoog and Spiekeroog (East Frisian Wadden Sea) by Becherer et al. (2016) showed that SPM did not derive from local erosion by tidal currents within the channel, but instead was the result of wind and wave-induced erosion on the surrounding tidal flats. These authors therefore assume that tidal channels are not the primary source for sediments in the Wadden Sea, but rather function as a distribution network for sediment that is eroded and deposited on the intertidal flats.

Our results indicate that increased SPM in the whole turbidity network during inundations strongly depends on the wind conditions, especially the conditions during the tides preceding an inundation (Figure 3-6). Constantly rising wind speeds over several tides from relatively stable southwestern directions are the conditions most likely to generate high SPM concentrations. Indeed, northern winds during inundation number 5 caused an average overall SPM reduction by $50 \%$ (Figure 3-6c, d). In general, significant fluctuations in wind speed and 
direction result in lower SPM concentrations. These results are consistent with Badewien et al. (2009), who showed a large impact of longer-lasting winds from relatively stable western wind direction on SPM concentrations in a tidal inlet between the East Frisian Islands of Langeoog and Spiekeroog.

On a large spatial scale, higher SPM concentrations on the tidal flats positively correlate with greater $M_{s}$ accumulation on the adjacent marshland (Figure 3-7). The conversion of $M_{s}$ accumulation into annual marshland accretion rates reveals that the three year (2016-2019) average accretion rate of $1.3 \pm 1.1 \mathrm{~mm} /$ a for Langeness is well in line with previous data from Schindler et al. (2014a). These values are much too low to compensate for the actual rise in the MHW of $5 \pm 0.3 \mathrm{~mm} / \mathrm{a}$. The comparison of the SPM concentrations on the tidal flat (possible SPM availability) with SPM on the marshland demonstrates that large amount of SPM cannot overtop the coastal barriers (Figure 3-8). Exploiting the full potential of SPM availability to be transported onto the marshland, for instance by adjusting the barrier heights would certainly increase the currently low accretion rates. On the basis of the SPM vs. $M_{s}$ correlation results (Figure 3-9) a tidal averaged SPM concentration threshold of at least $0.15 \mathrm{~g} / \mathrm{l}$ should be available on the tidal flat and entirely reach the marshland surface to allow for sufficient $M_{s}$ accumulation to keep pace with SLR. Based on the observations of individual inundations measured with the turbidity network, the SPM availability threshold is even exceeded in the tidal flat except at station T-2 where the tidal flat SPM does not reach the availability threshold, most likely due to its constricted position between spur dykes. Nevertheless, the present bulk exploitation of the SPM available on the tidal flat is as low as $20 \%$ at station T-8 and $9 \%$ percent at station T-7 on the marshland, at 50 and $150 \mathrm{~m}$ distance to the Hallig edge, respectively and therefore do not reach the needed SPM threshold concentrations (Figure 3-8).

To understand the low degree of SPM transfer onto the marshland of Langeness, the influence of the current coastal protection measures on SPM transport to the marshland has been investigated by comparing $M_{s}$ accumulations at three different locations with each showing locally different barrier heights (Figure 3-10). All locations show for all inundations consistently higher $M_{s}$ accumulations within the trap clusters behind the lower barriers (Figure 3-10). Besides overall higher $M_{s}$ accumulation, grain size analysis reveals coarser-grained sediment in the trap clusters behind the lower barriers, which additionally supports a less disturbed SPM transport onto the marsh surface. These findings clearly demonstrate the strong limitations in 
SPM transport raised by the height of the barriers. Generally, the finer fraction $<63 \mu \mathrm{m}$ is more able to reach the marshland than the most common grain size fraction between 63 and 200 $\mu \mathrm{m}$. On the other hand, this grain size fraction accounts for up to $70 \%$ within the sediment accumulators after an inundation and is predominantly present in tidal flat areas with stronger wind and tidal forcing. The sand-sized fraction apparently fails to overtop the coastal revetments resulting in a median grain size of all trap clusters of $32 \pm 11 \mu \mathrm{m}$ calculated from all eight inundations.

Between 2016 - 2019, the highest $M_{s}$ accumulation occurred in the winter of 2016/17, which coincided with the highest average inundation heights (Table 3-1). These results are consistent with Temmerman et al. (2003a), who demonstrated that sedimentation rates increase with increasing inundation heights. However, there was low correlation between SPM and $M_{s}$ with increasing inundation heights for the seasons $2017 / 18$ and $2018 / 19$, mostly likely due to small differences in inundation heights within each season (Table 3-1).

Grain size analysis of the sediment accumulator samples revealed variations in the composition of the tidal flat SPM (Figure 3-11) that cannot simply be explained by the prevailing weather conditions. The grain size distributions at station T-1 remained finer at inundation 6 than at inundation 3 (Figure 3-11, Figure 3-12) despite stronger wind and higher waves (Table 3-3). This can be explained by the resuspension of freshly deposited mud (Fettweis et al. 2010), which was deposited during preceding calm periods. Field observations during sampling of the sediment accumulators in the tidal flat provide supporting evidence: a one to four $\mathrm{cm}$ thick muddy layer referred to as "mobile layer" (Bartholdy and Aagaard 2001) of fine sediment was found on the tidal flat. At the subsequent sediment accumulator sampling after the next inundation, the muddy layer was eroded and no longer available on the tidal flat. The relevance of the remobilization of such muddy layers can be demonstrated by, for instance, inundations number 7 and 8 . These inundations followed each other after just 5 days (Table 3-3) without a distinct calming of the weather conditions in between. We assume that no muddy layer could be formed before inundation number 8 . The significant variability in wind conditions (Figure $3-6 e, f)$ and the absence of the muddy layer is likely the reason why we determined the lowest SPM availability and accumulation rates of all sampled inundations (Figure 3-7) at inundation number 8 . That means that such muddy layers are not formed when inundations follow each other rather quickly without intermediate calm periods. In addition, we often determined much higher SPM concentrations after longer relatively calm periods (e.g. see fourth tide before 
inundation number 5 in Figure 3-6c). This indicates that a muddy layer can favor higher $M_{s}$ accumulation on the marshland as this additional source of fine sediments on the intertidal flats increase the fine grained fraction of the available SPM, which is more able to cross the coastal barriers than the coarse grained fraction (Figure 3-11,Figure 3-12).

We are aware that even the site specific and well-calibrated turbidity measurement network has uncertainties in long-term analysis of SPM concentrations (e.g. Druine et al. 2018; Fettweis et al. 2019), especially in tidal flats where hydrodynamic conditions change rapidly within a tide. Nevertheless, we successfully achieved an assessment of the available SPM around Langeness by averaging the determined SPM concentrations on tidal scales. This is demonstrated by large spatial scale agreement between increased tidal flat SPM concentrations (SPM availabilities) and higher $\mathrm{M}_{\mathrm{s}}$ accumulation on the marshland (Figure 3-7) and small spatial scale correlation between SPM at the marshland stations and the adjacent accumulated $\mathrm{M}_{\mathrm{s}}$ (Figure 3-9).

\subsection{Conclusions and outlook}

Utilizing a network of eight autonomously operating turbidity measurement stations along with sediment accumulators and sediment traps, installed on the intertidal flats around as well as the supratidal marshland of Hallig Langeness (North Frisian Wadden Sea, southern North Sea), we could quantify the unexploited potential of SPM, which is available on the tidal flats but hindered by coastal revetments to be transported onto the marshland during inundations.

Based on the relations between the SPM at the marshland stations and the adjacent accumulated $M_{s}$ a SPM availability threshold of $0.15 \mathrm{~g} / \mathrm{l}$ should be at least available on the tidal flat to allow for sufficient transport of the available SPM onto the marshland to keep pace with SLR. This threshold has been exceeded on the tidal flat by all of the eight individual inundations measured within the turbidity network. However, the identified SPM at the marshland stations is on average only $20 \%$ of the total SPM availability in the southeastern area close to the Hallig margins and decreases to $9 \%$ with increasing distance to the margin. This demonstrates that the system is rather transport limited than material limited. An efficient transport of the 
sufficiently available SPM towards the marshland surface is probably somehow hindered by coastal protection barriers resulting in vertical accretion rates too low to compensate for SLR.

On the one hand, we successfully achieved an assessment of the available SPM around Langeness by averaging the determined SPM concentrations on tidal scales demonstrated by large spatial scale agreement between increased tidal flat SPM concentrations and higher $\mathrm{M}_{\mathrm{s}}$ accumulation on the marshland. On the other hand, we showed a small spatial scale correlation between SPM at the marshland stations and the adjacent accumulated $M_{s}$. The best conditions to generate high SPM availability on the tidal flats and high $M_{s}$ accumulation rates on the marshland are (i) constantly raising wind speed over several tides from stable southwestern directions until the inundation and (ii) calm periods before the inundation potentially provide a thin muddy layer of fine sediments as additionally local SPM source which are more able to reach the marshland surface due to its finer grain size distribution.

Grain size distributions of the available SPM on the tidal flat and the accumulated $M_{s}$ from all sediment traps on the marshland revealed that the most common sand-sized fraction between 63 and $200 \mu \mathrm{m}$ is not able to reach the Hallig surface. A detailed look by comparing $M_{s}$ accumulations at three different locations with locally differing barrier heights consistently demonstrated the limitations in SPM transport onto the marsh surface related to barrier height, i.e. behind higher barriers sediment accumulation is reduced along with a finer particle size distribution compared to the lower barriers.

According to our findings, coastal protection measures that are only bases on barriers and their elevations endanger the long-term preservation of the Halligen. This supports the current rethinking of coastal protection strategies. These findings are to be incorporated into current coastal protection measure strategies in order to support the long-term preservation of the North Frisian Halligen.

\section{Acknowledgements}

The study forms part of the joint research project "Real life coastal laboratory to develop short-

and long term strategies for the protection and preservation of Schleswig-Holsteins Halligen (Living Coast Lab Hallig)" funded by the German Federal Ministry of Education and Research 
(BMBF, 03F0759C). We wish to thank the inhabitants of Langeness for their hospitality and helpfulness in supporting the research project. We thank the responsible authority for coastal protection, national parks and marine protection in Schleswig Holstein (LKN.SH) for providing topographical, sea state and gauge level data. We thank the German Meteorological Service (DWD) for providing wind data. 


\section{$4 \quad$ Assessing sediment accumulation at inundated anthropogenic marshland in the southeastern North Sea: using particle tracking on modified coastal protection structures}

This paper is published 2021 in Ocean \& Coastal Management, 208 (2021), 1-13

Doi: https://doi.org/10.1016/j.ocecoaman.2021.105631

Ingo Hache ${ }^{1}$, Sebastian Niehüser ${ }^{2}$, Volker Karius ${ }^{1}$, Arne Arns $^{3}$, Hilmar von Eynatten ${ }^{1}$

Corresponding author: Ingo Hache, University of Göttingen, Geoscience Center, Goldschmidtstr.

3, 37077 Göttingen, Germany, ingo.hache@uni-goettingen.de

${ }^{1}$ Geoscience Center University of Göttingen, Department of Sedimentology and Environmental Geology, Goldschmidtstraße 3, 37077 Göttingen, Germany

${ }^{2}$ University of Siegen, Research Institute for Water and Environment, Paul-Bonatz-Straße 9-11, 57076 Siegen, Germany

${ }^{3}$ University of Rostock, Faculty of Agricultural and Environmental Sciences, Justus-von-LiebigWeg 6, 18059, Rostock, Germany

Keywords: Sediment transport, Inundation, Vertical accretion, Particle tracking, Coastal protection 


\section{Abstract}

Adaptation of protection structures to the continuously rising sea level is a major topic at many coastal areas all over the world. In shallow marine environments like tidal flats these structures often aim at mitigation rather than prevention of inundations. These anthropogenic interventions, however, are prone to disturb sediment transport onto regularly inundated marshlands. This applies to ten island-like marsh areas called Halligen in the southeastern North Sea, off the coast of Schleswig-Holstein, Germany. The capability of these marsh surfaces to keep pace with sea level rise depends on vertical accretion rates due to the accumulation of suspended sediments that reach the Hallig surface during annually occurring inundations. According to latest findings, the current coastal protection structures such as revetments, dikes and breakwaters represent an obstacle for sediment transport, which intensifies the imbalance between sea level rise and vertical accretion rates on the Halligen.

A detailed hydrodynamic model of the largest Hallig Langeness and its surrounding tidal flats is applied to investigate adjustments to impermeable breakwater constructions for different inundation scenarios. The sediment transport from the tidal flats towards the marshland and the subsequent accumulation is modelled by Lagrangian particle tracking. The particle tracking results indicate almost doubling of accumulated particles by decreasing the breakwater heights from $2.56 \pm 0.3 \mathrm{~m}$ to a constant height of $1.92 \mathrm{~m}$ in the moderate inundation scenario. In comparison, storm surges conditions show an increase of particle accumulation by a factor of 6.9. The results further delineate a potential increase of the recently measured vertical accretion rates by almost an order of magnitude, given an appropriate design and management of the protection structures.

\subsection{Introduction}

Coastal regions around the world have to deal with manifold challenges imposed by the ongoing sea level rise (SLR) and likely consequences such as increasing coastal flooding (Seneviratne et al. 2012), increase in the frequency of extreme events (Hunter 2009) and higher storm surge water levels (Arns et al. 2015a). These consequences are particularly evident for low lying tidal marsh areas where 20 to $50 \%$ may get drowned by SLR until the end of this 
century (e.g. McFadden et al. 2007; Craft et al. 2009). Tidal marshes are among the most valuable ecosystems on Earth (Costanza et al. 1997) which highlight the urgent need to mitigate or even hamper the challenges caused by global and regional SLR for these areas.

Marsh areas have a limited adaptability to keep pace with SLR (e.g. Kirwan et al. 2010; D'Alpaos et al. 2011; Kirwan et al. 2016a, 2016b). Each tidal cycle that inundates the marsh surface provides suspended particulate matter (SPM) that can accumulate on the marsh surface. This repeating process leads to a vertical accretion of the marsh surface (e.g. Schuerch et al. 2012, 2013; Schindler et al. 2014a). For natural marshes, the interaction primarily depends on tidal variations (Temmerman et al. 2003a, 2003b), the availability of SPM and the rate of SLR (e.g. Temmerman et al. 2004). This interaction is disrupted by human interventions, for instance by conversion of coastal marshlands to agriculture and aquaculture (Kirwan and Megonigal 2013) or due to typical coastal protection measures such as dikes, sea walls and embankments. As a result, the latter intervention as well as their continuous heightening and widening to keep up with SLR can block the wave- and wind-driven supply of sediments along coastlines (e.g. Temmerman et al. 2013), which increase the limitations in adaptation to SLR.

The so-called Halligen in the southeastern North Sea are ten small anthropogenic modified island-like marshlands located in a highly dynamic tidal flat area (Figure 4-1) In fact, the Halligen constitute remnants of two catastrophic floods in the $14^{\text {th }}$ and $17^{\text {th }}$ century. With an average elevation of only few decimeters above mean high water (MHW) the Halligen and their inhabitants would be exposed to SLR and annually occurring inundations (e.g. Deicke et al. 2007) without coastal protection measures. The protection measures for the marshland started in 1914 with small dike constructions on the largest Halligen Hooge and Langeness (Müller and Fischer 1917). Heavy storm surges and a continuous SLR led to the construction of additional protection measures like revetments and breakwaters which enclose the larger Halligen and protect parts of the smaller Halligen. Today's vertical accretion rates of three of the larger Halligen $(1.5 \pm 0.9 \mathrm{~mm} / \mathrm{a}$ for Hooge, $2.6 \pm 0.9 \mathrm{~mm} / \mathrm{a}$ for Nordstrandischmoor and $1.2 \pm 0.8$ $\mathrm{mm} / \mathrm{a}$ for Langeness) clearly show that none of these marshlands keep pace with the local MHW rise of $5.0 \pm 0.3 \mathrm{~mm} / \mathrm{a}$ (Schindler et al. 2014a). This imbalance underlines the urgent need for new measures to maintain the Halligen for the future.

For this reason, the interdisciplinary joint research project "Living Coast Lab Halligen" was launched which includes the development of strategies to mitigate the imbalance between SLR 
and vertical accretion. In this framework we examined the SPM supply to the Halligen within three winter seasons from autumn 2017 to spring 2020 (data for the last season are still in progress) using an unique autonomous working turbidity measurement network at Hallig Langeness (Hache et al. 2019). Within eight individually monitored inundations from the first two winter seasons we uncovered a five times higher untapped SPM availability in the tidal flats around the Hallig compared to the Hallig marshland, i.e. a huge part of the available SPM is not transported onto the marsh surface during inundations (Hache et al. 2020). The study also demonstrated the impact of artificial structures (e.g. breakwaters) on limiting sediment accumulation on the marsh surface. In a previous study by Schindler et al. (2014a), it is believed that lower coastal protection structures (e.g. Schindler and Willim 2015) are among others the cause for increased vertical accretion rates at Hallig Nordstrandischmoor in comparison to Hallig Hooge and Langeness. This work can therefore be seen as a first model approach with the use of particle tracking in gaining insight into the possibilities of increasing the natural adaptation potential of the Halligen through lowering coastal protection structures in view of global SLR.

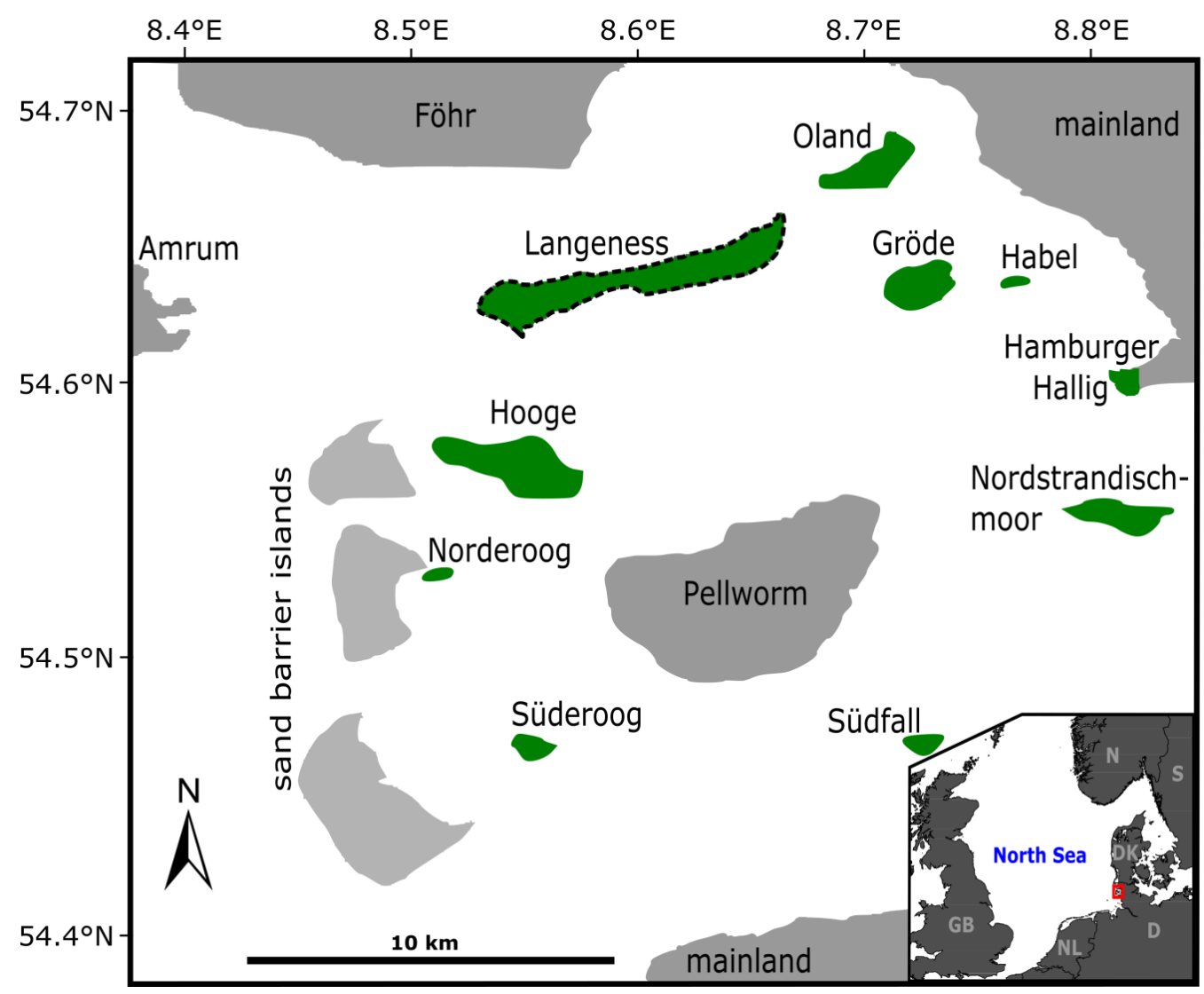

Figure 4-1: The map shows the ten Halligen (green). The study area Langeness (black dashed outline) is surrounded by the islands of Föhr, Amrum and Pellworm and the mainland of Schleswig Holstein (northern Germany) as well as nine other Halligen and three sand barrier islands. The inset shows the location of the area within the southeastern North Sea and the neighboring countries: GB = Great Britain, NL = The Netherlands, D = Germany, DK = Denmark, S = Sweden, N = Norway. 
The use of particle tracking to model and analyse sediment dynamics has become a state-ofthe-art method. Thonon et al. (2007) already investigated sediment deposition patterns and rates at the lower river Rhine in the Netherlands by using particle tracking. Allison et al. (2017) modelled particle paths and their accumulation spots by releasing a fine (silt) and a coarse (sand) particle class into the Mississippi Delta which were tracked up to the Gulf of Mexico. A 2D hydrodynamic numerical model was used by Liubartseva et al. (2018) to examine drifted and accumulated plastic particles in the Mediterranean Sea. They were able to detect significant accumulation spots along coastlines and the sea floor and locate the most contaminated areas and highly polluted local patches. Particle tracking was also used by Stanev et al. (2019) to study the propagation pathways of marine litter in the North Sea. The particle tracks helped to explain the abnormal transport of wooden and GPS drifters initiated by an extreme wind event in 2018, which caused a reversal of the circulation at the sea surface as well as in deeper layers.

In this paper we use a two-dimensional hydrodynamic-numerical model and a Lagrangian particle transport module to investigate the possibilities in making adjustments to the coastal protection structures for increased efficiency of transporting the available SPM from the tidal flats onto the Hallig surface. Specifically, we use the Danish Hydraulic Institute's (DHI) Mike FM (flexible mesh) model suit. With a detailed 2D hydrodynamic numerical model from Langeness and the surrounding tidal flats, we are able to make adjustments to the breakwaters of Langeness and examine their impact on SPM transport. Because of only moderate inundations with relatively low water levels in the two measuring seasons 2017/18 and 2018/19 used here, it was not possible to monitor an extreme storm surge and its impact on SPM availability, transport and accumulation so far. We simulate the well observed moderate inundations with different adjustments to the coastal revetment heights. To also examine higher peak water levels and its effect on accumulation quantities, we used data of the historic storm surge on November 24, 1981 and performed particle tracking with the same coastal revetment adjustments as taken for the moderate inundations. 


\subsection{Material and Methods}

\subsubsection{Study Area}

Hallig Langeness is the largest of the ten existing Halligen with a total area of $9.24 \mathrm{~km}^{2}$ and is located in the North Frisian Wadden Sea off the coast of the federal state of Schleswig-Holstein (Germany). The terrain height of Langeness has an average absolute ground level of $0.17 \mathrm{~m}$ above MHW without artificial structures. Protection measures like dikes encompass the entire Hallig with an average height of $0.98 \mathrm{~m}$ above MHW (Schindler and Willim 2015) to protect Langeness from minor floodings, especially during the summer season. Revetments and water impermeable breakwaters, further named as breakwaters, have been installed almost everywhere along the margin of Langeness to mitigate erosion by tidal flow induced currents and wave impact. In addition, spur dikes have been installed to reduce flow induced erosion by tidal currents. Larger inundations during storm surges are more likely to occur in the winter months when harsh weather conditions are common. In such cases, the man-made dwelling mounts (called "Warfen") with an average elevation of approximately $3.6 \pm 0.5 \mathrm{~m}$ above MHW provide shelter to the 113 inhabitants (2018), their cattle and goods. Protection levels were updated in 2000 and further increases are already implemented due to the continuous SLR (Hofstede 2019). Tidal gates at the outlets of channels draining the Hallig impede minor flooding and ensure fast dewatering after inundations. The tidal system is semidiurnal with an average mean tidal range of $2.7 \mathrm{~m}$ measured at tide gauge Hilligenley in the SW of Langeness (see Figure 4-2b) during the winter seasons 2017/18 and 2018/19. Such range is characteristic for a mesotidal regime according to Hayes (1979).

\subsubsection{Numerical model set-up}

In a first step, an existing two-dimensional barotropic hydrodynamic model of the entire North Sea including parts of the North Atlantic by Arns et al. (2015b) is used to create a model chain for simulating the hydrodynamic conditions around and on the Hallig Langeness (Figure 4-2a). The boundary conditions used and the calibration and validation of the model results are explained in detail by (Arns et al. 2015b; Arns et al. 2017). The model is adapted according to the latest available bathymetric, tidal and atmospheric boundary conditions. The main adjustment is the meteorological forcing at the boundary conditions. In order to also take the observed inundation events recorded during the period between 2017 and 2019 into account, 
updated atmospheric reanalysis data are required. Therefore, the atmospheric data of the ERA5 project of the "European Centre for Medium-Range Weather Forecasts" (ECMWF) are used. The ERA5 dataset $(C 3 S, 2017)$ consists of the reanalysed and homogenised combination of an atmospheric model, observations from satellites and weather stations worldwide on a grid with a resolution of about $31 \mathrm{~km}$. For the hydrodynamic model forcing the air pressure as well as the $\mathrm{u}$ - and v-components of the wind speed from the ERA5 data set are used. The temporal resolution of the meteorological data is 60 minutes.

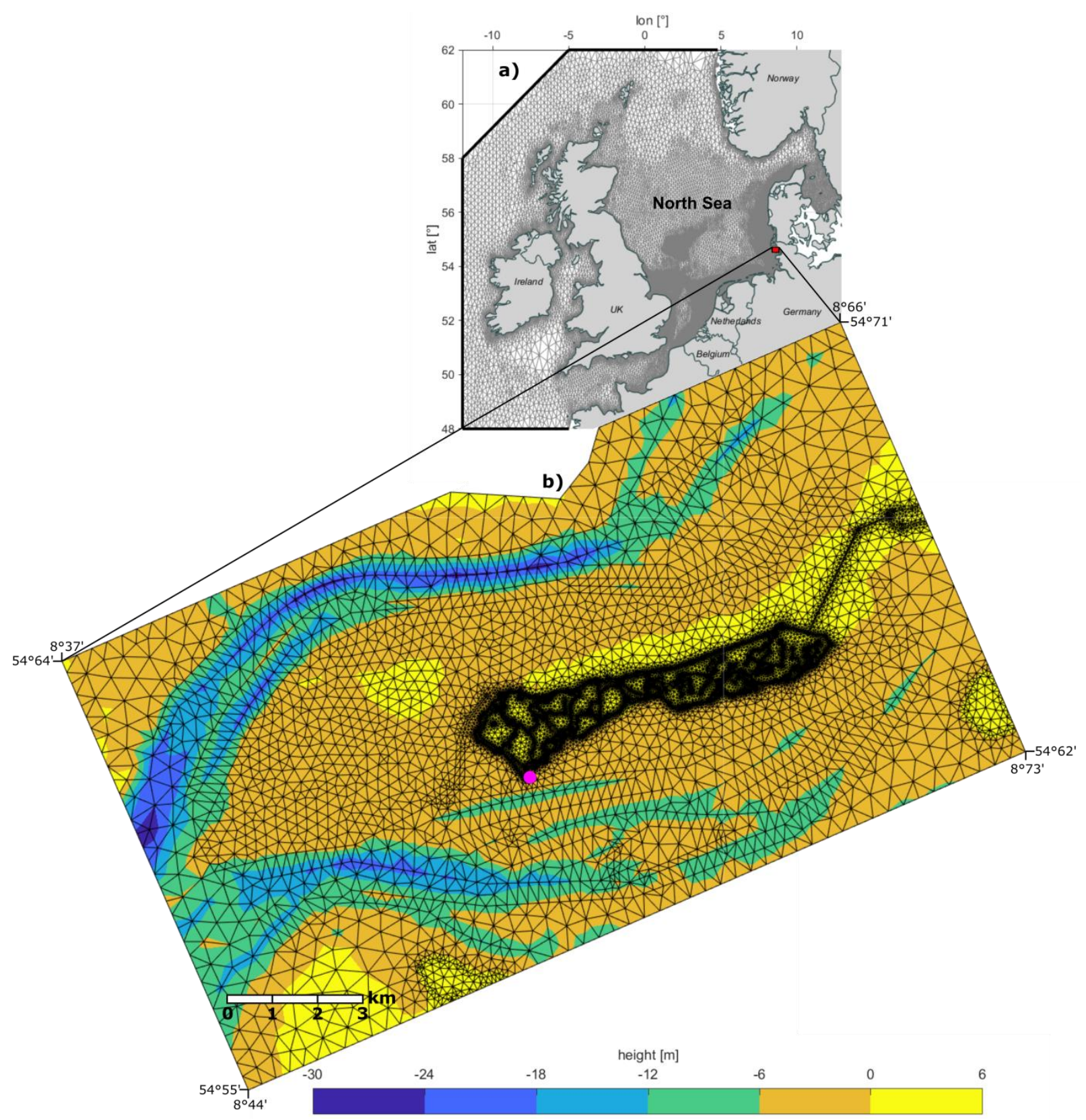

Figure 4-2: (a) Hydrodynamic model of the entire North Sea including parts of the North Atlantic by Arns et al. (2015b). The red square shows the location of the high resolution model of the Hallig Langeness (b) including topographic elevation in meter above mean sea level. The magenta dot marks the location of the tide gauge Hilligenley. 
The model of the North Sea provides the hydrodynamic boundary conditions for a second, highresolution model of the Hallig Langeness (Figure 4-2b). In order to simulate the required water level and sea state information, a two-dimensional, depth-integrated, barotropic hydrodynamic model is developed, considering the dynamic interaction between tides and the meteorologically induced surge (see e.g. Arns et al. 2020). The model is created with the software MIKE21 ${ }^{\circledR}$ from the Danish Hydraulic Institute (DHI) (e.g. Warren and Bach 1992). The model is based on the numerical solution of the two-dimensional shallow water equations, in the form of the depth-integrated, incompressible Reynolds-averaged Navier-Stokes equations, assuming a hydrostatic pressure distribution. Thus, the model consists of continuity, momentum, temperature, salinity and density equations. The spatial discretization of the equations is performed with a cell-centered finite volume method (DHI, 2017b). In addition to the hydrodynamics module, the MIKE21 ${ }^{\circledR}$ "Spectral Wave Model FM (MIKE 21 SW)" is used to simulate wind-waves according to the "wave action conservation" equation (DHI, 2017b).

The topography of the Hallig Langeness with the surrounding tidal flats of the North Frisian Wadden Sea in the detailed model is based on different data sets. The surface of the Hallig Langeness and the adjacent tidal flats are considered by digital terrain models from 2005 and 2014 on a Cartesian grid with a resolution of 1 m (DGM1). Furthermore, survey data from 2014 collected during construction measures are included. The data were provided by the responsible authority for coastal protection, national parks and marine protection of SchleswigHolstein (LKN.SH). Uncovered areas of the tidal flats are supplemented with bathymetric charts of 2012 from the research project "AufMod" (Heyer and Schrottke 2013) funded by the German Coastal Engineering Research Council (KFKI). For a detailed integration of the trench system and breakwaters in the mesh the building catalogue of the LKN.SH is used.

A moderate inundation and a strong inundation (i.e. as consequence of a storm surge) are simulated with a spin-up time of one week. The model results finally form the basis for the simulation of sediment transport for the most important sediment fractions found in the sediment samples after inundations. The suspended sediment samples on tidal flats reach median grain sizes of approximately $80 \mu \mathrm{m}$ and sediment accumulation samples on the marshland surface reach median values of $30 \mu \mathrm{m}$ (Hache et al. 2020). Here, the particle tracking module "PT" of MIKE21 ${ }^{\circledR}$ is used to simulate the suspended sediment particles. The module uses the Lagrangian discrete parcels method to model drift, dispersion, sedimentation, buoyancy, and remobilization, among others. The simulated particles can be assigned to 
individual classes, each of which can be assigned different properties with regard to decay, buoyancy and remobilization (DHI, 2017a). Due to the considered particle classes with 30 and $80 \mu \mathrm{m}$, particle tracking features such as decay and flocculation are neglected and a constant settling velocity is adjusted for each particle class. Because of the simulated hydrodynamic scenarios up to storm surges, horizontal and vertical dispersion are neglected as well. The remobilization is parameterized by setting a critical shear stress for each particle class within the erosion feature. As soon as the critical bed shear stress acts on a respective particle, it is set in motion. If this process takes place again after the particle had previously settled, the particle is remobilized. The particle tracks thus only consider the remobilization of previously released particles to the model within the individual hydrodynamic scenario and therefore neglects erosion processes of consolidated sediments entirely. By using the module, the paths of the individual particles as well as their sedimentation and remobilization as effected by the hydrodynamic conditions can be identified. A total of 94 sources (see Figure 4-3) are used to continuously add one particle per time step ( 1 minute) at each source to the model. This enables each particle to be assigned to a source location during the entire inundation scenario from which it was released. The evaluation interval of the particle tracks for each individual source takes place every 10 minutes. A particle is referred to as 'particle transport' as soon as a particle is drifted from the tidal flat towards the coastal protection structures and reach the marshland surface. If particles are moved from the marshland back to the tidal flat during the same inundation scenario, these particles are referred to as 'particle loss'. Only the particle quantities of the particle transport that remain on the marshland surface until the inundation is over are referred to as 'particle accumulation'. 


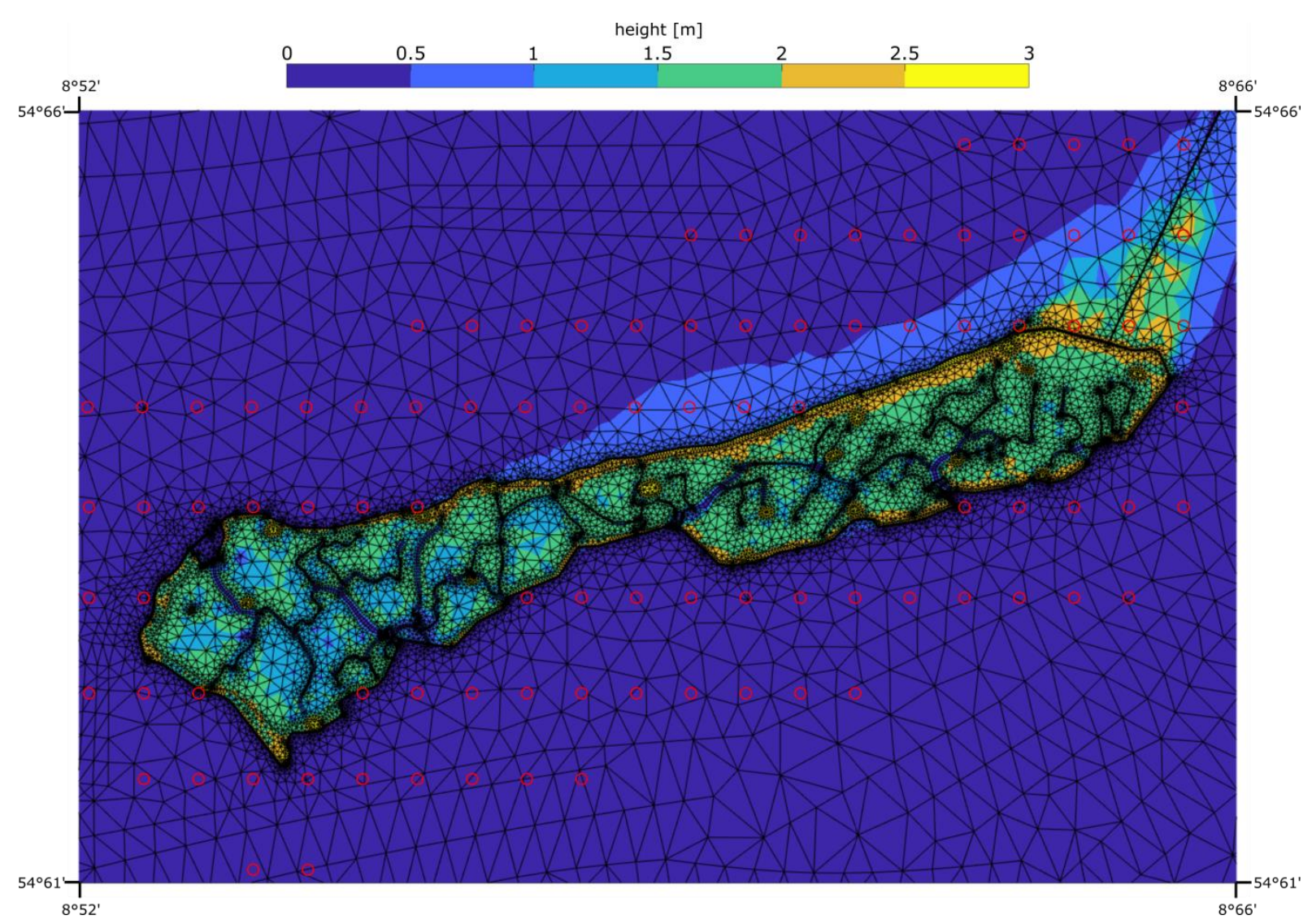

Figure 4-3: Close up of the high resolution model of the Hallig Langeness surrounded by 94 sources from which particles are added including topographic elevation in meter above mean sea level.

\subsubsection{Hydrodynamic simulations}

The local inundations are usually caused by increased surges from strong westerly winds superimposing on high astronomical tides. The strength and duration of the prevailing wind conditions have therefore a large impact on available SPM (detailed in Hache et al. 2020) and the inundation height and thus distinguish a moderate inundation from a storm surge. For the storm surge classification, we refer to water levels of the German Federal Maritime and Hydrographic Agency (BSH). Accordingly, on the German North Sea coast a storm surge is characterized by water levels between 1.5 to 2.5 meters above MHW. We refer to the nearby tide gauge Dagebüll $\left(54.731^{\circ} \mathrm{N}, 8.687^{\circ} \mathrm{E}\right.$ monitored by the Federal Waterways and Shipping Administration) with a determined MHW of 1.42 meter above MSL. On March 5, 2019, Langeness was inundated by an event that met the requirements for a moderate inundation with a peak high water level of $1.21 \mathrm{~m}$ above MHW. To generate the hydrodynamics for a moderate inundation we chose to start the simulation period on February 26, 2019. To generate peak water levels that met the requirements for a storm surge, we chose to start the 
simulation period on November 17, 1981 to capture the severe storm surge on November 24, 1981, where a peak high water level of about $3.3 \mathrm{~m}$ above MHW occurred.

\subsubsection{Particle classes and parametrization}

In Hache et al. (2020), the evaluation of all sediment traps on Langeness from 8 individual moderate inundations resulted in a median grain size of D50 $=32 \pm 10.9 \mu \mathrm{m}, \mathrm{D} 10=3.3 \pm 0.8 \mu \mathrm{m}$ and $\mathrm{D} 90=107 \pm 19.9 \mu \mathrm{m}$ and a standard deviation of $\mathrm{SD}=54.4 \pm 25.5 \mu \mathrm{m}$. We also used suspended sediment accumulators in the tidal flat around Langeness to determine which sediments would be available as a source for transport onto the Hallig during an inundation. The grain sizes of the suspended sediment accumulator samples were always coarser and reached D50 $=83.2 \pm 15.5 \mu \mathrm{m}, \mathrm{D} 10=6.9 \pm 1.3 \mu \mathrm{m}, \mathrm{D} 90=159.2 \pm 6.5 \mu \mathrm{m}$ and a standard deviation of SD $=56.3 \pm 1.4 \mu \mathrm{m}$ at a more wind and wave exposed area in the southeast of Langeness within the turbidity network. The coarser grain size distribution of the suspended sediment on tidal flats around Langeness compared to the finer accumulated sediment on the marsh surface indicates an obstacle for sediment transport and an unused availability of suspended sediments that does not reach the Hallig surface, at least during moderate inundations. We therefore investigated the sediment transport by using the particle classes of $30 \mu \mathrm{m}$ and $80 \mu \mathrm{m}$ in diameter for our particle track simulations (Table 4-1) to capture the representative range of verifiably measured grain sizes at moderate inundations. Representative grain size measurements during storm surges could not been carried out so far due to a lack of storm surges in the turbidity measurement period from March 2017 to March 2019. Therefore, an Increase in grain sizes of transported particles towards the marsh surface can only be assumed during storm surges, but is not considered in this work due to the lack of reliable grain size data of storm surges. 
Table 4-1: Two particle classes and their used parametrization for the particle tracking.

\begin{tabular}{ccccc}
\hline Particle & Particle & Settling & Critical bed & Mass [g] \\
class & diameter $\mathbf{d}$ & velocity $\mathbf{w}_{\mathrm{s}}$ & shear stress & \\
& {$[\mu \mathrm{m}]$} & {$[\mathrm{mm} / \mathrm{s}]$} & $\tau_{\mathrm{ce}}\left[\mathrm{N} / \mathrm{m}^{2}\right]$ & \\
\hline 1 & 30 & 1 & 0.433 & $3.7 \mathrm{E}-08$ \\
2 & 80 & 3.2 & 0.641 & $7.02 \mathrm{E}-07$ \\
\hline
\end{tabular}

All particles are attributed the density of quartz with $\rho_{\mathrm{s}}=2.65 \mathrm{~g} / \mathrm{cm}^{3}$. The settling velocity $\mathrm{w}_{\mathrm{s}}$ of the $80 \mu \mathrm{m}$ particle is calculated according to Soulsby (1997) from the density $\rho_{w}$ and the kinematic viscosity $v$ of water with a salinity of 28 per mill and $5{ }^{\circ} \mathrm{C}$ as well as the grain diameter $\mathrm{d}$ and the gravity $g$ using the dimensionless grain diameter $D^{*}$ resulting in $3.2 \mathrm{~mm} / \mathrm{s}$ (Table $4-1$ ).

$$
\begin{gathered}
D_{*}=\left[\frac{g \cdot\left(\left(\frac{\rho_{s}}{\rho_{w}}\right)-1\right)}{v^{2}}\right]^{\frac{1}{3}} \cdot d \\
w_{S}=\frac{v}{d}\left[\left(10,36^{2}+1,049 \cdot D_{*}^{3}\right)^{\frac{1}{2}}-10,36\right]
\end{gathered}
$$

Because the validity of the $w_{s}$ formula for settling velocities is restricted to particle sizes down to the fine end of $62 \mu \mathrm{m}$, we assume a settling velocity of $1 \mathrm{~mm} / \mathrm{s}$ for the $30 \mu \mathrm{m}$ particles according to Whitehouse et al. (2000).

The critical bed shear stress $\tau_{\mathrm{ce}}$ is calculated from the critical shield parameter $\Theta_{\text {ce }}$ according to the Shields' curve fit by Brownlie (1981), the acceleration of gravity g, the respective grain diameter $D$ and the densities of water $\rho_{w}$ and sediment particles $\rho_{s}$ (detailed in DHI 2017a).

$$
\tau_{c e}=\Theta_{c e} \operatorname{gD}\left(\rho_{s}-\rho_{w}\right)
$$

\subsubsection{Particle track simulation cases}

For the comparison of the SPM transport between a moderate inundation and a storm surge, no changes in the breakwater heights are made in order to obtain the current particle transport conditions (case $A$ in Figure 4-4). In previous field studies, measured sediment accumulations behind different breakwater heights demonstrated that more sediment accumulate behind lower breakwaters (Hache et al. 2020). Therefore we adjusted the heights of the higher 
breakwaters to the neighboring lower breakwaters at two locations, which were investigated for sediment accumulation rates within individual sampled inundation events in the last winter seasons from autumn 2017 to spring 2020 (case B in Figure 4-4). Consequently, we reduced the western breakwater heights from $2.79 \pm 0.01$ to a constant height of $2.6 \mathrm{~m}$ and in the southeast from $2.86 \pm 0.12$ to a constant height of $1.92 \mathrm{~m}$. In case $C$, we adjusted the breakwater heights along the entire southern coastline to the same height adjustment made in the southeast in Case B. That reduced the breakwater heights from $2.56 \pm 0.3 \mathrm{~m}$ to a constant height of $1.92 \mathrm{~m}$ (case $C$ in Figure 4-4). After each adjustment, an updated simulation of the hydrodynamics was necessary.

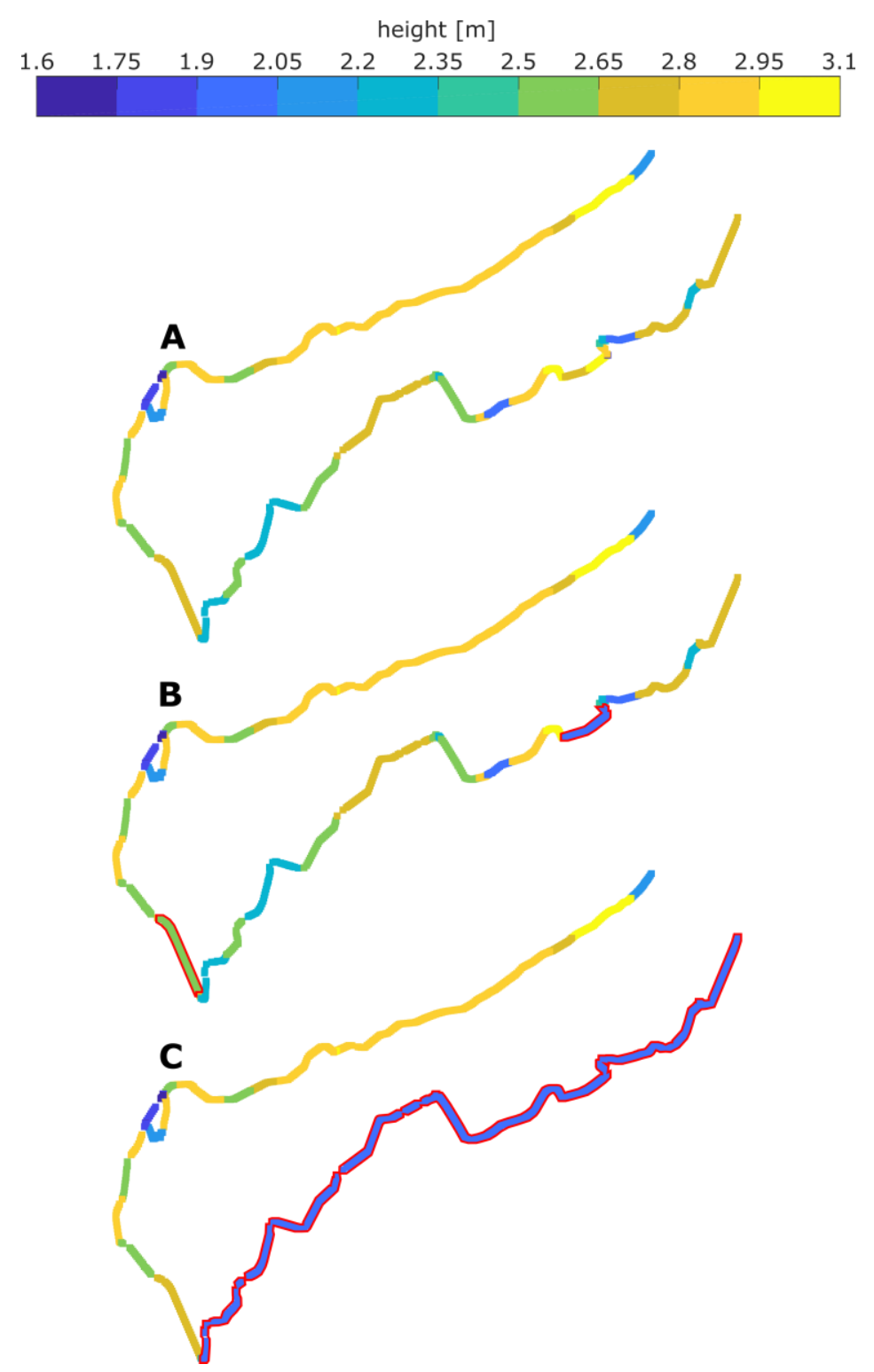

Figure 4-4: Breakwater height adjustments at Hallig Langeness chosen for three particle track simulation cases $(A, B$ and $C$ ) showing in meter above mean sea level. The red framed sections in case $B$ and $C$ show the areas where the heights were adjusted to lower values compared to case $A$. 


\subsection{Results}

\subsubsection{Hydrodynamics}

Figure 4-5 shows the tidal cycles from the hydrodynamic simulations of the moderate inundation and the storm surge, calculated at the location of the tide gauge Hilligenley on Langeness. The last of the respective tidal cycles shows the tide that inundates Langeness, indicated by the grey line in Figure 4-5 that represents the mean breakwater height of $2.34 \mathrm{~m}$ above MSL for Langeness (Schindler and Willim, 2015). The moderate inundation simulation reaches a peak high water level of $2.72 \mathrm{~m}$, which corresponds to $1.3 \mathrm{~m}$ above MHW. The storm surge simulation reaches a peak high water level of $3.25 \mathrm{~m}$, which corresponds to $1.83 \mathrm{~m}$ above MHW.

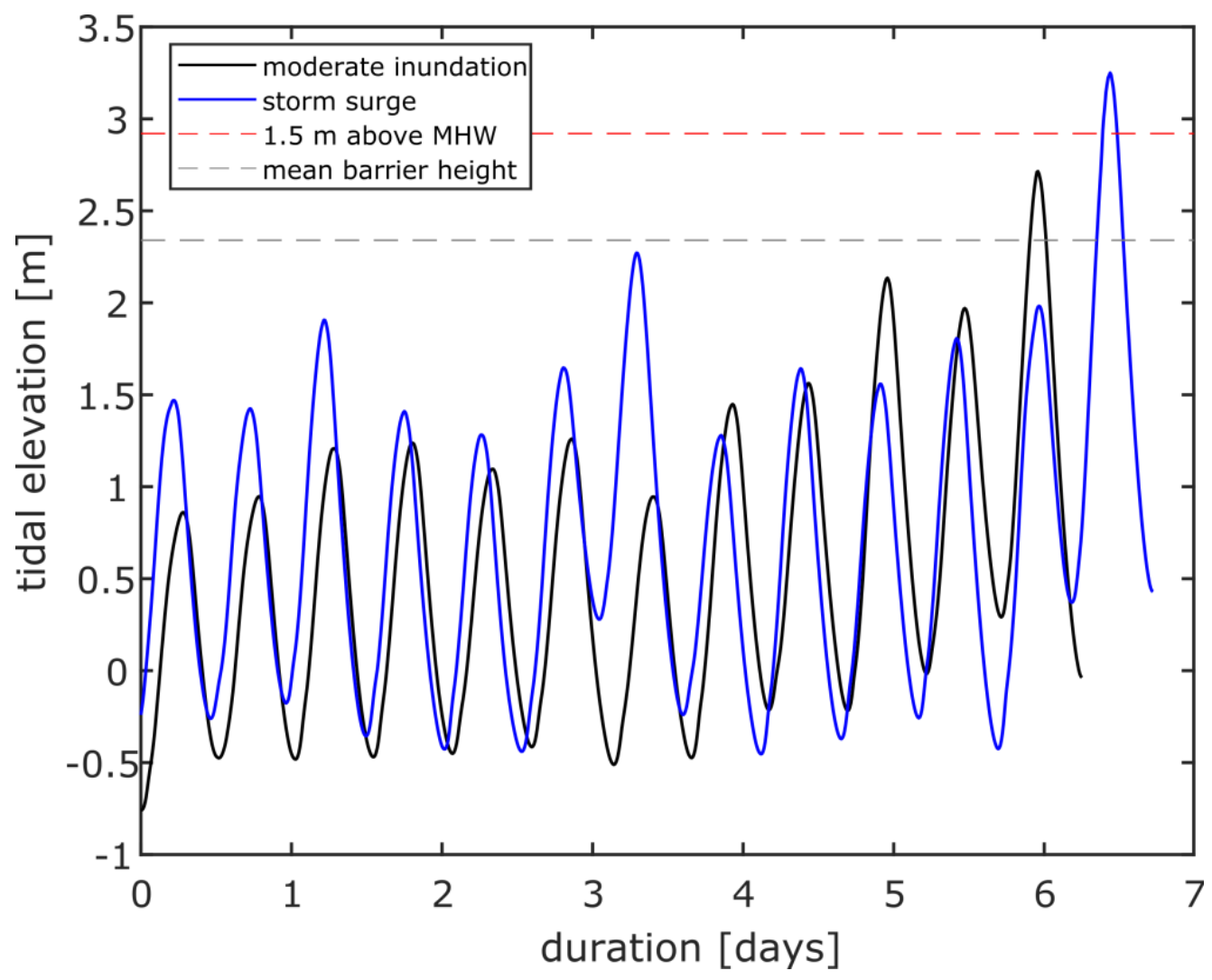

Figure 4-5: Tidal cycles from the hydrodynamic simulations of the moderate inundation (black line) and the storm surge (blue line) which end up in the tidal cycle that inundates Langeness, calculated at the location of the tide gauge Hilligenley (see Figure 4-2b). The dashed red line shows the reference height for water levels from which a storm surge occurs according to the German Federal Maritime and Hydrographic Agency (BSH). The dashed grey line shows the mean breakwater height of Langeness according to Schindler and Willim (2015). 


\subsubsection{Particle tracks $30 \mu \mathrm{m}$}

The comparison of the moderate inundation and the storm surge without making any adjustments in the breakwater heights (A cases) clearly shows a strong increase in the numbers of $30 \mu \mathrm{m}$ particles that accumulate area-wide on Langeness (Figure 4-6).
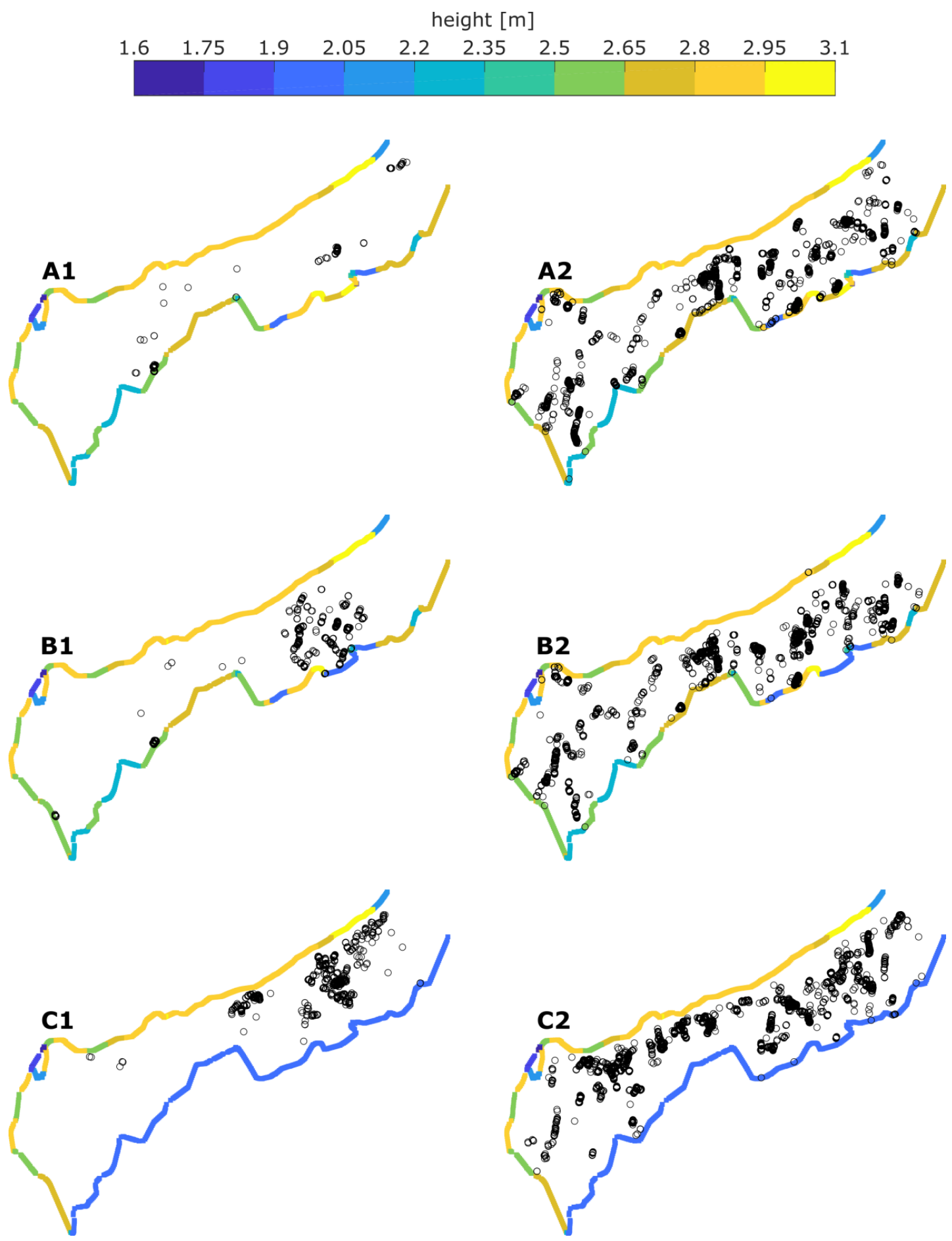

Figure 4-6: Particle class $1(30 \mu \mathrm{m})$ track simulation results of accumulated particles on Langeness including the breakwater heights in meter above mean sea level. The left side shows the moderate inundations from top to bottom (A1, B1, C1) of all breakwater adjustments $A$ to $C$ in Figure $4-4$ and the right side shows the storm surge event in the same order (A2, B2, C2). 
In comparison to the moderate simulation case A1, $590 \%$ more particles have been accumulated during the storm surge simulation A2 if only the $30 \mu \mathrm{m}$ particles are taken into account (Figure 4-7, Table 4-2). In contrast to the moderate inundation, there is also a significant loss of particles represented by the difference between the total number of transported particles and the number of accumulated particles that have actually remained on the Hallig surface throughout the entire inundation event. The quantity of the particle loss in case $A 2$ is almost twice the accumulated particle quantity of the moderate event $A 1$. Particle accumulation in case A2 would have increased by $777 \%$ if loss of particles could be hampered completely.

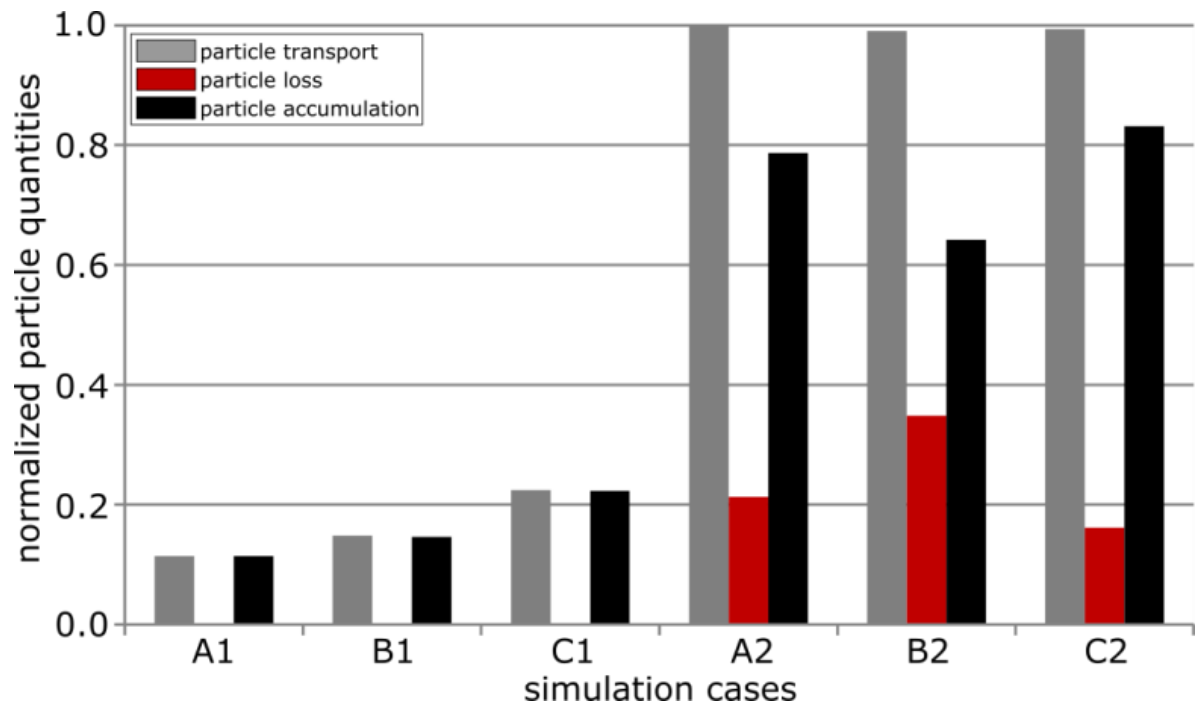

Figure 4-7: Bar chart showing particle transport, loss and accumulation quantities in case of $30 \mu \mathrm{m}$ particle size, for moderate inundations (A1, B1, C1) and storm surge simulations (A2 B2 C2), normalized to the particle transport of simulation $\mathrm{A} 2$. 
Table 4-2: Particle accumulation quantities and their ratios $(80 \mu \mathrm{m} / 30 \mu \mathrm{m})$ of all simulation cases from A1 to $\mathrm{C} 2$ from both particle classes.

\begin{tabular}{|c|c|c|c|}
\hline simulation & accumulated & accumulated & accumulated \\
\hline \multirow[t]{3}{*}{ case } & particle & particle & particle \\
\hline & quantity [30 & quantity [80 & ratio 80 \\
\hline & $\mu \mathrm{m}]$ & $\mu \mathrm{m}]$ & $\mu \mathrm{m} / 30 \mu \mathrm{m}$ \\
\hline A1 & 1131 & 13 & 0.01 \\
\hline B1 & 1452 & 0 & 0 \\
\hline C1 & 2212 & 61 & 0.03 \\
\hline$A 2$ & 7803 & 3499 & 0.45 \\
\hline B2 & 6368 & 3375 & 0.53 \\
\hline C2 & 8249 & 5566 & 0.67 \\
\hline
\end{tabular}

In case of a moderate inundation, the limited breakwater height adjustments (B1) increase the accumulated particles by $28 \%$ compared to the original case A1 (Figure 4-7). The particle locations in Figure 4-6 show that the increase is restricted to the adjusted area at the southeastern breakwater because the adjustment in the southwest does not noticeably increase the number of accumulated particles in the western part of Langeness. The storm surge case (B2) shows a relatively uniform particle distribution over the entire Hallig, similar to A2 (Figure 4-6), but a significant decrease in the number of accumulated particles by $18 \%$ in comparison to A2 (Figure 4-7). However, a high proportion of particle loss in B2 (35\% of the transported particles) reduces the accumulated particles significantly.

The lowering of the breakwater heights on the entire southern side of the Hallig (case $C$ ) leads to a $96 \%$ increase of the particle accumulation for the moderate inundation simulation (C1 vs. A1 in Figure 4-7). But still, the increase in accumulation is strongly focused on the eastern part (Figure 4-6). The storm surge case $C 2$ is characterized by the smallest particle loss of all three storm surge simulations, resulting in the highest particle accumulation (Figure 4-7). The particle distribution pattern covers both eastern and western part of the Hallig but particle deposition is shifted to the north relative to A2 and B2 (Figure 4-6). 


\subsubsection{Particle tracks $80 \mu \mathrm{m}$}

The particles with a median diameter of $80 \mu \mathrm{m}$ hardly reach the surface of Langeness during moderate inundations. For this particle class, no further particle distributions as in Figure 4-6 is shown because they do not provide additional information. Based on the total accumulated number of both particle classes $(30 \mu \mathrm{m}$ and $80 \mu \mathrm{m})$, the $80 \mu \mathrm{m}$ particles achieved negligible proportions of around $1 \%$ in case $\mathrm{A} 1$ and $0 \%$ in case B1 and are therefore not shown in (Figure 4-8). Only simulation case C1 shows a slightly higher proportion of accumulated $80 \mu \mathrm{m}$ particles up to $2.7 \%$. In comparison to these small numbers, storm surge conditions results in significantly higher quantities of accumulated $80 \mu \mathrm{m}$ particles (Figure 4-8).

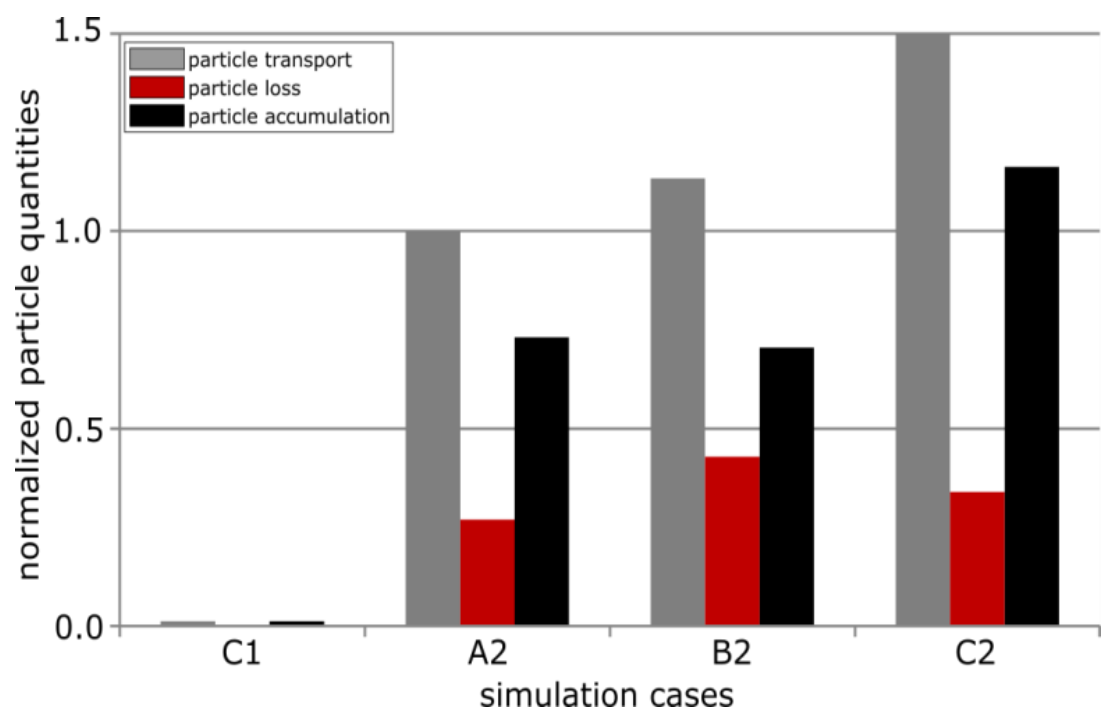

Figure 4-8: Bar chart showing particle transport, loss and accumulation quantities in case of $80 \mu \mathrm{m}$ particle size, for moderate inundation (C1) and storm surge simulations (A2 B2 C2), normalized to the particle transport of simulation A2.

The storm surge simulations $A 2$ to $C 2$ show a similar picture compared to the $30 \mu \mathrm{m}$ simulation cases. Case C2 displays again the highest particle accumulation while case B2 shows again a significant decrease in particle accumulation due to the highest particle loss of all $80 \mu \mathrm{m}$ simulation cases. In contrast to the $30 \mu \mathrm{m}$ particle simulations with almost no difference in the total particle transport of cases A2, B2 and C2 (Figure 4-7), $80 \mu \mathrm{m}$ particle simulations show an increase of $50 \%$ in particle transport for C2 compared to A2. The particle accumulation ratios of $80 \mu \mathrm{m}$ to $30 \mu \mathrm{m}$ particles also increase from 0.45 in case $A 2$ to 0.53 in case B2 up to 0.67 in case C2 (Table 4-2). 


\subsubsection{Particle accumulation versus peak high water level}

In order to expand the data set of simulated inundation events between moderate inundations and storm surges assumption we simulate five more inundations with different peak high water levels (Table 4-3) and without any adjustments to the breakwaters (A cases) and evaluating the particle tracks with $30 \mu \mathrm{m}$ particles. The correlation result between peak high water and particle accumulation of all seven inundations resulted in a coefficient of determination after Pearson $\left(R^{2}\right)$ of 0.87 with a $p$-value of 0.002 and a root mean square error of 0.14 (Figure 4-9).

Table 4-3: All seven simulated inundation events with the two key parameters for the linear regression analysis in Figure 4-9; particle accumulation quantities $(30 \mu \mathrm{m})$ normalized to the particle accumulation of simulation A2 (inundation event 1 ) and peak high water levels. The observed peak high water levels are records from tide gauge Hilligenley except inundation event 1 which was recorded by tide gauge Dagebüll. The inundation events printed in bold are storm surges.

\begin{tabular}{ccccc}
\hline inundation event & occurred on & normalized & simulated peak & observed peak \\
& & particle & high-water level & high-water level \\
& & accumulation & quantities & \\
[m] $]$ & \\
\hline $\mathbf{1}$ & 24.11 .1981 & 1 & 3.25 & 4.72 \\
$\mathbf{3}$ & 06.12 .2013 & 0.6 & 3.18 & 3.3 \\
4 & 13.09 .2017 & 0.4 & 2.7 & 2.63 \\
5 & 05.03 .2017 & 0.14 & 2.72 & 2.64 \\
6 & 11.02 .2018 & 0.07 & 2.48 & 2.69 \\
7 & 08.12 .2017 & 0.06 & 2.6 & 2.62 \\
\hline
\end{tabular}




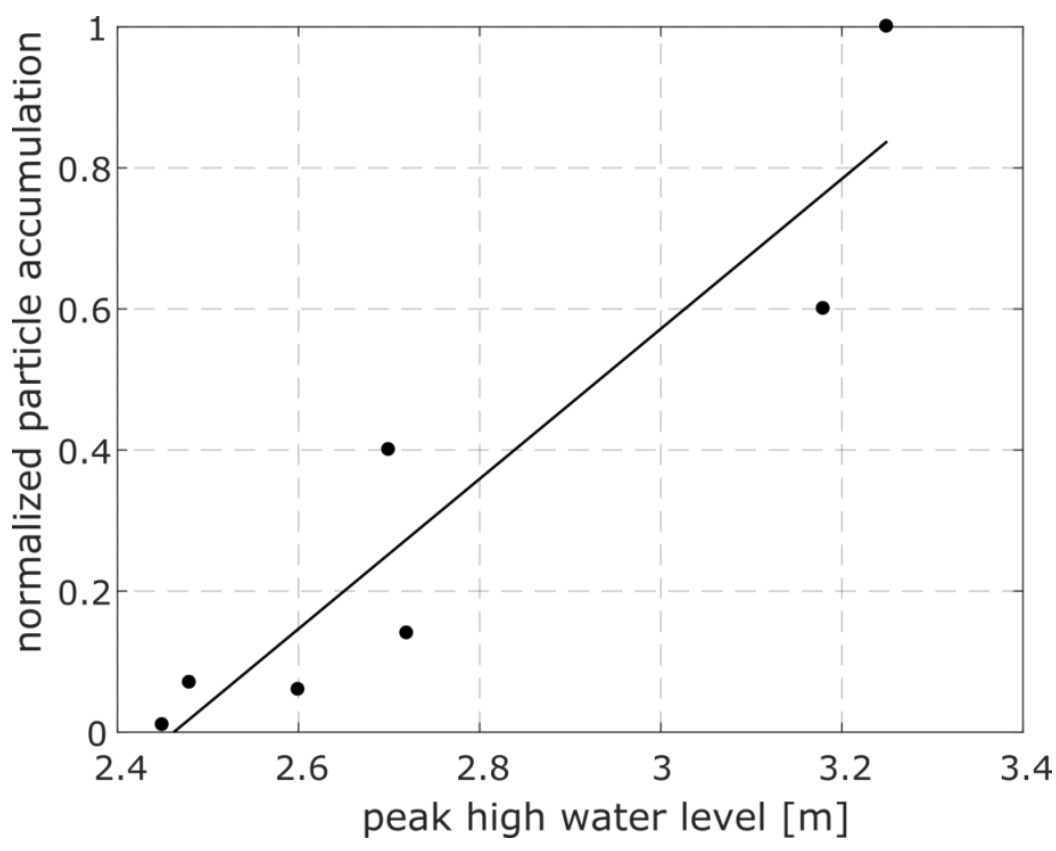

Figure 4-9: Linear regression analysis of seven individual modelled inundations (Table 4-2) between simulated peak high water levels and normalized particle accumulation quantities to the particle accumulation of simulation A2 (inundation event 1 in Table 4-2) resulted in a coefficient of determination after Pearson $\left(R^{2}\right)$ of 0.87 with a $p$-value of 0.002 and a root mean square error of 0.14 .

\subsection{Discussion}

The two hydrodynamic simulations indicated in Figure 4-5 caused peak high-water levels that met the requirements of a moderate inundation $(<1.5 \mathrm{~m}$ above $\mathrm{MHW})$ and a storm surge $(>1.5$ $\mathrm{m}$ above MHW) according to the storm surge classification of the BSH. The storm surge water levels were simulated using the astronomical and meteorological conditions of November 24, 1981 as input. However, simulated water levels were $1.83 \mathrm{~m}$ above MHW while observed water levels were more than $3 \mathrm{~m}$ above MHW during this event. These differences are mainly from the superordinate hydrodynamic model which tends to underestimate individual storm surge water levels (Arns et al. 2015b), especially at historical extremes where the meteorological reanalysis loses confidence. When simulating moderate inundations, differences between simulated and observed peak high-water levels are significantly smaller. Thus, the absolute deviation between simulated peak high-water levels and observed peak high-water levels within four simulated moderate inundations yield $0.13 \pm 0.04 \mathrm{~m}$ on average compared to three simulated storm surges with an average deviation of $0.99 \pm 0.44 \mathrm{~m}$ (also see Table 4-3). It is therefore important to mention that we are not interested in reproducing these particular 
events, but rather to simulate exemplary conditions during a moderate inundation and a storm surge that meet the requirements of the above mentioned peak water level on which particle tracking can be carried out.

The simulation results of the moderate inundation without adjustments to the breakwater heights (case A1) indicates that only the finer particles with $30 \mu \mathrm{m}$ grain size reach the Hallig surface and coarser particles with $80 \mu \mathrm{m}$ mainly remain in front of the Hallig margin (Figure 4-7 and Figure 4-8). This is in line with grain size measurements for eight individual moderate inundations in Hache et al. (2020). These showed a median grain size of $32 \pm 11 \mu \mathrm{m}$ in all sediment traps on Langeness. In contrast sediment samples collected in front of Langeness reached three times higher values. These median grain sizes are within a range of $32 \mu \mathrm{m}$ to 137 $\mu \mathrm{m}$ depending on spatial and temporal varying local hydrodynamic forcing e.g. a low energetic area due to a shielded position between two spur dikes or a high energetic area at a more wind and wave exposed area (Hache et al. 2019). Adjusting the breakwater heights at moderate inundations shows an almost two-fold increase in the $30 \mu \mathrm{m}$ particle accumulation (Figure 4-7), whereas the increase in $80 \mu \mathrm{m}$ particles remains negligibly small. The increase in the $30 \mu \mathrm{m}$ particles can therefore primarily be explained by an earlier flooding and therefore longer flood phase towards the Hallig surface (Table 4-4). However, due to the lower inundation heights and the resulting weaker hydrodynamic conditions at moderate inundations, there is almost no possibility to transport $80 \mu \mathrm{m}$ particles (though available) onto the Hallig by decreasing the breakwater heights. Even in simulation case C1 where we drastically decrease the breakwater heights from southwest to southeast, the $80 \mu \mathrm{m}$ particles only achieved a very small proportion below $3 \%$ from the total number of both accumulated particle classes.

The storm surge shows an almost 7-fold increase in $30 \mu \mathrm{m}$ particle accumulation compared to the moderate inundation, even without any breakwater height adjustments (Figure 4-7). This can be explained by the distinctly higher water level along with significantly stronger hydrodynamic conditions. This assumption is in line with our correlation results between rising peak high water levels and increasing accumulated particle quantities of seven individual simulated inundations (Table 4-3, Figure 4-9). This observation is supported by the simulation results by Temmerman et al. (2003a) showing higher accumulation rates with higher water levels. Reports of the Hallig inhabitants also point to high sediment loads visible from surface deposits right after storm surges. 
During storm surges particle transport and accumulation is strongly increased, however, in contrast to moderate inundations there are high particle loss quantities especially in simulation case B2 (Figure 4-7 and Figure 4-8). The differences in flow velocities between case A2 and B2 are predominant locally distributed in the area of the adjusted breakwaters (Figure 4-10). The high flow velocities are most clearly visible in the southeast where the barrier height was decreased. This flow channeling effect is most likely the reason that in comparison to simulation case $A 2$ particle remobilization instead of particle accumulation takes place close to the adjusted breakwater (case B2, Figure 4-6). In comparison to case B, the incoming and outgoing water at the breakwater adjustment in case $\mathrm{C}$ is distributed more widely on the entire southern edge which avoids distinct flow channeling (Figure 4-11). This reduced flow also enables particle accumulation close to the adjusted breakwater (case C2, Figure 4-6). Therefore, in simulation case $\mathrm{C} 2$, the loss of particles decreases and results in the highest particle accumulation quantity of all simulation cases (Figure 4-7 and Figure 4-8).

Considering the $30 \mu \mathrm{m}$ particle transport of all storm surge cases (Figure 4-7), it becomes apparent that the particle quantities transported are almost the same. This suggests that the availability of $30 \mu \mathrm{m}$ particles during storm surges may be completely utilized. Only the particle loss seems to be the decisive factor for the amount of $30 \mu \mathrm{m}$ particle accumulation on the Hallig surface, which is highest for case C2. Notably, particle loss at the storm surge simulations is predominantly higher than particle accumulation at the moderate inundations. In other words, preventing particle loss during storm surges would be equivalent in terms of sediment accumulation to two moderate inundations of case A1 or B1. These results clearly underline previous suggestions for changes in protection measures or in the handling of these measures like managing the tidal gates (Schindler 2014; Hofstede et al. 2019b). Such measures should aim at slowing down the outflowing water after inundations to substantially increase the proportion of particles that settle on the Hallig surface. These additional measures to slowing down the drainage water flow are especially relevant as on the one hand broad scale adjustments to the breakwater increase the particle transport duration during the flood phase but on the other hand decreases the inundation duration after slack water (Table 4-4).

Considering the $80 \mu \mathrm{m}$ particle transport, however, there is a steady increase in the quantities of transported particles with more extensive reductions in breakwater heights (Figure 4-8). This suggests a hampering influence of the breakwater heights on the transport of coarse particles during storm surge conditions. The existing limiting effect of the breakwater heights on $30 \mu \mathrm{m}$ 
particle transport processes at moderate inundations (Figure 4-7) seems thus shifted to the coarser particles under storm surge conditions, when the $30 \mu \mathrm{m}$ particles are fully transported onto the Hallig.

In addition, the evaluation of the particle tracks indicate that the particles that accumulate on the Hallig came almost exclusively from the south. Particles released from northern sources did not cross the northern margin of Langeness. For this reason, adjustments of the northern breakwater heights did not achieved higher quantities of accumulated particles, but rather contributed to an increase in particle loss. This observation agrees with averaged accretion rates by Schindler et al. (2014a), which show the highest accretion rate in the southeast of Langeness and generally low values along the northern Hallig margin. These low accretion rates would therefore not solely be the result of high breakwaters in the northern part, but rather occur due to the particle transport directions prevailing there. Adjustments of the breakwater heights in order to reach the highest increase in particle accumulations would make more sense in the southern and especially in the southeastern part of Langeness, where we already determined the highest SPM variabilities (Hache et al. 2019). We also identified an SPM low spot on the tidal flat in the southwest of Langeness in which the lowest SPM concentrations of the entire turbidity measurement network were measured during all inundations because of its sheltered position between two spur dikes. The vast majority of spur dikes (39 out of 51) are in the west of Langeness (Figure 3, in Hache et al. (2019)) which underlines the low particle deposition in the western part of Langeness during all moderate simulation cases where hydrodynamic conditions are generally weaker than at storm surges ( $A 1$ to $C 1$ in Figure 4-6).

Moreover, the large-scale adjustment of the entire southern breakwater (case $C$ ) led to a significant particle accumulation shift to the north relative to the simulation cases $A$ and $B$ at the moderate inundation and especially at the storm surge case ( $C 1$ and C2 in Figure 4-6). In order to enhance an area wide sediment distribution on the Halligen, the adjustment of the southern margin would be a promising approach. Today, the highest topographical regions of the larger Halligen Hooge and Langeness lay directly behind the Hallig margin (see Figure 4-3). This finding accords well with the high accretion rates in that regions (Deicke et al. 2007; Schindler et al. 2014a). SPM measurements during individual moderate inundations on Hallig Langeness showed an SPM concentration decrease of more than $50 \%$ between two stations with different distances (50 and $150 \mathrm{~m}$ ) from the Hallig margin supporting the assumption of a 
transport limited distribution of particles (Hache et al. 2020). Applying the theoretical increase to the monitored winter season in 2018/19 with only moderate inundations highlights the significance of the investigated breakwater adjustments. Five moderate inundations resulted in an average annual vertical accretion rate of $1 \mathrm{~mm}$ (Hache et al. 2020). Assuming that the $30 \mu \mathrm{m}$ particle increase generated by the breakwater adjustments would apply to the vertical accretion rate in the same way, results in an vertical accretion to 1.3 or $2 \mathrm{~mm}$ in case $\mathrm{B}$ or $\mathrm{C}$, respectively. Consequently, for the breakwater adjustments simulated here, moderate inundations are not able to generate sufficiently high vertical accretion rates to compensate the observed MHW rise of $5 \mathrm{~mm} / \mathrm{a}$. However, the breakwater adjustments are suitable to better exploit the existing potential of the untapped sediment availability around Langeness which at least mitigates the imbalance between vertical accretion rate and rising $\mathrm{MHW}$. The simulation results further indicate that the sediment most commonly available on the tidal flats is fine sand (represented by the $80 \mu \mathrm{m}$ particles), which is transported onto Langeness during storm surge conditions only. Replacing the five monitored moderate inundations in 2018/19 (A1 cases) by 5 storm surges of similar strength than the simulated one (A2 cases), would theoretically result in a vertical accretion of $6.9 \mathrm{~mm}$ by only taking the $30 \mu \mathrm{m}$ particle accumulation increase into account. Replacing the moderate A1 cases by storm surge C2 cases, would theoretically result in a vertical accretion of $7.3 \mathrm{~mm}$. In total, an additional accretion rate of $1.4 \mathrm{~mm}$ by preventing particle loss in simulation cases C2 would increase the theoretically accretion rate up to $8.7 \mathrm{~mm}$. The storm surge scenarios accordingly generate the highest vertical accretion rates, which may be capable to compensate the postulated MHW rise according to the model but still has to be proven with ongoing SPM measurements during storm surges around Langeness.

Please note that the particle loss quantities considered here refers only to the actually transported particles and does not consider erosion of already consolidated sediments. The erosion of sediment that accumulated during previous years is considered as very local feature and largely negligible for investigations considering the entire Hallig. Furthermore, the influence of vegetation on sediment trapping due to a reduction in turbulent kinetic energy (Mudd et al. 2010) is not considered in the model. Adapted vegetation, therefore, may be a suitable component as a reduction in breakwater height locally increases flow velocity (Figure 4-10 and Figure 4-11), decreases the total inundation time (Table 4-4) and might increase erosion processes on the marsh edge. We emphasize that these influencing factors of the 
marshland vegetation on vertical accretion rates should be implemented and further investigated in future models.

In general, however, it can be argued that the current coastal protection strategy to protect the Halligen from SLR does not promise a long-term success due to the implicit limitations on vertical accretion rates. Sticking to this strategy it would be necessary to continuously raise the breakwaters to keep pace with SLR (e.g. Temmerman et al. 2013), which in turn would further impair the natural adaptability of the Halligen.

\subsection{Conclusions}

Particle track simulations using various scenarios and two particle size classes (30 $\mu \mathrm{m}$ and 80 $\mu \mathrm{m}$ in diameter) allow to investigate the limiting influence on particle transport caused by coastal protection measures like dikes and breakwaters. Adjustment of the breakwater heights result in an up to $96 \%$ increase of accumulated $30 \mu \mathrm{m}$ particles under moderate inundation conditions. Under these conditions, however, the adjustments do not significantly increase the amount of $80 \mu \mathrm{m}$ particle. Only storm surges are able to transport appreciable amounts of the $80 \mu \mathrm{m}$ particles onto the Hallig surface. The transport of $80 \mu \mathrm{m}$ particles increases up to $50 \%$ by reducing the breakwater heights. Thus, the potential of available suspended sediment is currently not fully utilized, even at storm surges. Moreover, significant proportions of the sediment that is transported onto the Hallig during a storm surge either do not settle or are remobilized and washed off the Hallig. Such particle loss during storm surges is often higher (up to factor 2) than the total quantity of accumulated particles at moderate inundations. Using the simulation results to theoretically increase the accretion rates of some moderate inundations measured in the past indicates that typical frequencies of moderate inundations are not able to compensate the imbalance between a MHW rise of $5 \mathrm{~mm} / \mathrm{a}$ and accretion rates of up to 2 $\mathrm{mm} / \mathrm{a}$ resulted by the tested adjustments in the breakwater heights. Storm surges, however, are able to generate the necessary accretion rates according to the model. Replacing the moderate inundations measured in the past by storm surges, would theoretically result in a vertical accretion of $6.9 \mathrm{~mm}$ and up to $7.3 \mathrm{~mm}$ with adjustments in the breakwater height. An additional vertical accretion increase of $1.4 \mathrm{~mm}$ by prevent particle loss would increase the 
theoretically accretion rate to $8.7 \mathrm{~mm}$ in total. Making better use of the sediment accumulation potential of the rarer and stronger events thus seems highly relevant.

The modelling results point out that narrow adjustment in the breakwaters may increase the loss of particles by drastically increasing the local flow velocities at the Hallig surface.

In this study we utilized a novel approach by using particle tracking within a detailed hydrodynamic model of an anthropogenic marshland and the surrounding tidal flats, which offers new insights into the impact of coastal protection structures on sediment transport. This approach can be applied to other regions where anthropogenic interventions are suspected to influence sediment accumulation rates. It is important to emphasize that a previous field study on available suspended sediments (i.e. grain size, SPM) on tidal flats and the resulting spatial sediment accumulation rates on the marshland, as well as their influencing factors and local conditions is essential to ensure the validity of this approach.

\subsection{Appendix}

Table 4-4: Inundation durations of the marshland during the flood-and ebb phase and the sum of both phases from the moderate inundation and storm surge simulation cases (see e.g. Figure 4-6) in minutes measured at the location of the interior tide gauge Kirchwarf on Langeness $\left(54.640^{\circ} \mathrm{N}, 8.614^{\circ} \mathrm{E}\right)$. The standard deviation is always \pm 10 minutes due to the chosen evaluation interval. The flood phase contains the duration where water level crosses the mean ground elevation of Langeness ( 1.53 m.a.m.s.I.) according to Schindler and Willim (2015) until peak high water level is reached. The duration of the following ebb phase contains the water levels from peak high water to the highest reduction in the breakwater height to $1.9 \mathrm{~m}$ above mean sea level. This assumption is made because the subsequent drainage water flow below $1.9 \mathrm{~m}$ above mean sea level only takes place through the tidal gates and to avoid potential bias caused by subsequent rising tides especially after the storm surge.

\begin{tabular}{cccc}
\hline Simulation cases & Flood duration [min] & Ebb duration [min] & Total inundation duration [min] \\
\hline A1 & 50 & 470 & 520 \\
B1 & 40 & 470 & 510 \\
C1 & 70 & 320 & 390 \\
A2 & 90 & 540 & 630 \\
B2 & 90 & 530 & 620 \\
C2 & 110 & 380 & 490 \\
\hline
\end{tabular}




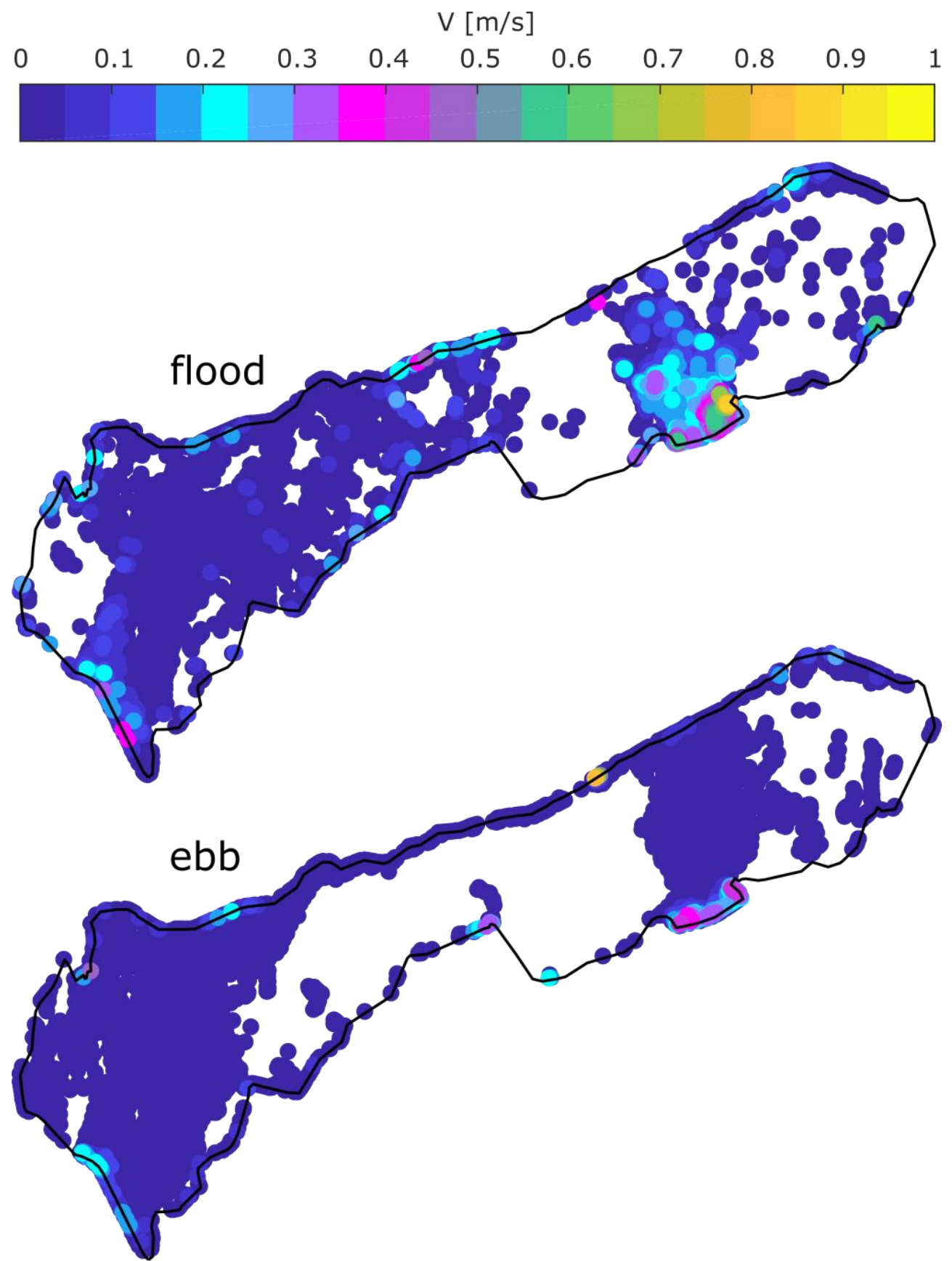

Figure 4-10: Scatter plot of the difference in the resulting flow velocities on Langeness between storm surge simulation A2 and B2 averaged during the flood and ebb phase. The white areas on Langeness show areas where no higher flow velocities occur due to the breakwater height adjustments (see Figure 4-4). 


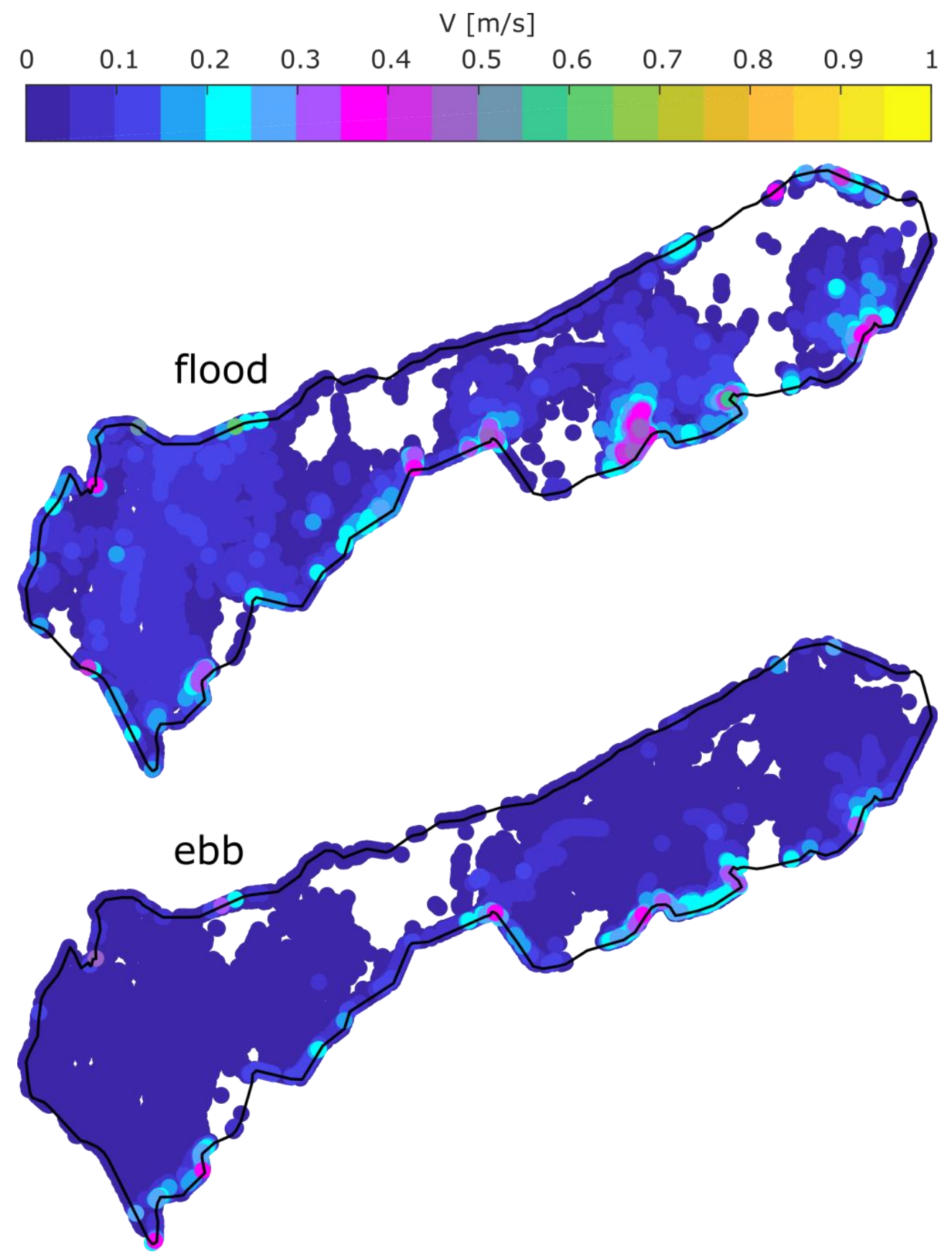

Figure 4-11: Scatter plot of the difference in the resulting flow velocities on Langeness between storm surge simulation A2 and C2 averaged during the flood and ebb phase. The white areas on Langeness show areas where no higher flow velocities occur due to the breakwater height adjustments (see Figure 4-4). 


\section{Acknowledgements}

The study forms part of the joint research project "Real life coastal laboratory to develop shortand long term strategies for the protection and preservation of Schleswig-Holsteins Halligen (Living Coast Lab Hallig)" funded by the German Federal Ministry of Education and Research (BMBF, 03F0759C). We wish to thank the inhabitants of Langeness for their hospitality and helpfulness in supporting the research project. We thank the Landesbetrieb für Küstenschutz, Nationalpark und Meeresschutz Schleswig Holstein (LKN.SH) for providing topographical data. The software package MIKE21 ${ }^{\circledR}$ was used for the hydrodynamic simulations and particle tracking, which was kindly provided by DHI-WASY. 


\section{Storm surge induced sediment accumulation on marsh islands in the southeastern North Sea: Implications for coastal protection}

This paper is submitted 2021 to Estuarine, Coastal and Shelf Science

Ingo Hache, Volker Karius, Hilmar von Eynatten

Corresponding author: Ingo Hache, University of Göttingen, Geoscience Center, Goldschmidtstr.

3, 37077 Göttingen, Germany, ingo.hache@uni-goettingen.de

Geoscience Center University of Göttingen, Department of Sedimentology and Environmental

Geology, Goldschmidtstraße 3, 37077 Göttingen, Germany

Keywords: Storm surge, Suspended particulate matter, Coastal protection, Sediment transport, Sediment accumulation, Vertical accretion 


\section{Abstract}

In view of global sea level rise (SLR), onshore transport of suspended particulate matter (SPM) in tide-dominated shallow marine systems is essential to generate vertical accretion on adjacent coastal lowlands during inundations. This is especially relevant for supratidal marshlands where access to sufficient SPM from the nearby intertidal flats might be limited by anthropogenic interventions such as dykes, revetments and tidal gates. Ensuring sufficient vertical accretion rates to balance SLR in such areas forms a challenging task for coastal protection strategies. With the use of an autonomously operating turbidity measuring network installed at and around a marsh island in the North Frisian Wadden Sea (Hallig Langeness, Germany), SPM concentrations were analysed for seven inundations caused by storm surges in the winter season $2019 / 20$. Their high energetic conditions in terms of water level, wave height and wind speed induced 3.1 to 3.5 fold higher SPM concentrations on the tidal flats compared to previously investigated moderate inundations at lower energetic conditions. During moderate inundations, SPM concentrations of the water on the Hallig marshland are reduced to $20 \%$ of the SPM concentration on the tidal flat while this proportion increases up to $73 \%$ for storm surges. Higher water levels and stronger hydrodynamic forcing at storm surges seem to reduce the limiting effects of coastal protection measures on sediment accumulation on adjacent marshlands. It is demonstrated that storm surge induced sediment accumulation is capable to generate vertical accretion rates on the marshland that keep pace with recent SLR. The vertical accretion rates in 2019/20 might have been even increased by $27 \%$ if the SPM that remains in the drainage water after inundations could be made useful for additional sediment accretion. Exploiting the full potential of vertical accretion of marshlands by SPM from recurrent inundations might be achieved by adjusted coastal management strategies.

\subsection{Introduction}

Global and regional sea level rise (SLR) and its likely consequences such as an increasing frequency of extreme events (Hunter 2009), increasing coastal flooding (Seneviratne et al. 2012) and higher storm surge water levels (Arns et al. 2015a) affect the conservation and natural adaptation capacity of intertidal flats and supratidal marshland areas. Therefore, the 
utilization and preservation of these areas has become a major topic in coastal research (Kirwan et al. 2010, 2016a,2016b; Kirwan and Megonigal 2013; D'Alpaos et al. 2011; Schuerch et al. 2012, 2013, 2018; Schindler et al. 2014b, 2014a; Hache et al. 2020). Considering that 20 to $50 \%$ of low lying marsh areas may get drowned by the end of this century (e.g. McFadden et al. 2007; Craft et al. 2009) underlines the need to mitigate or hamper the consequences for these ecosystems, which are among the most valuables on earth (Costanza et al. 1997). This challenge includes the so-called Halligen, ten small island-like, anthropogenically modified marshlands located in the tidal flats of the southeastern North Sea (North-Frisian Wadden Sea, Germany; Figure 5-1).

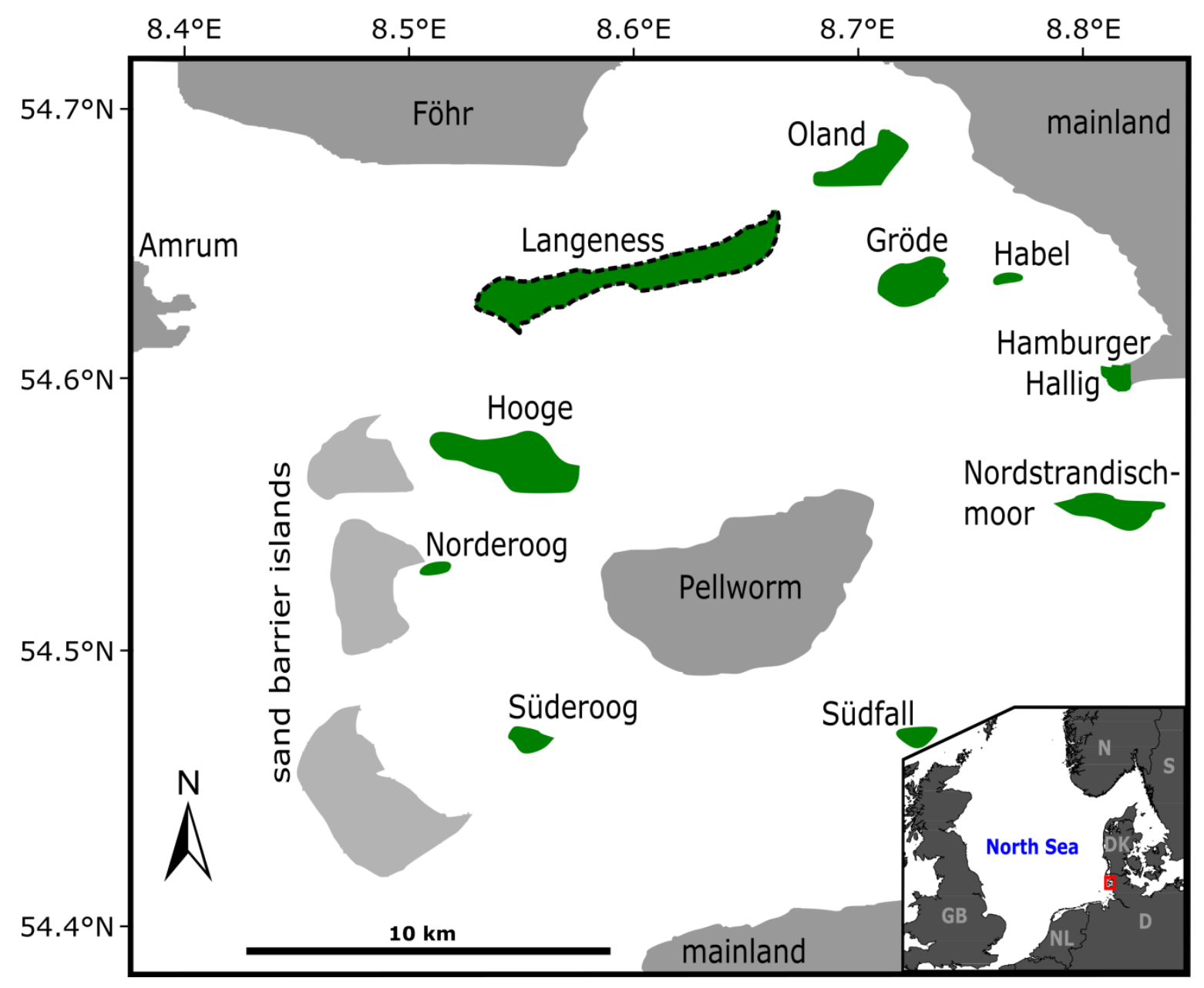

Figure 5-1: Map of the ten Halligen (green), located in the North Frisian Wadden Sea in the southeastern North Sea. The study area Langeness (black dashed outline) is surrounded by the islands of Föhr, Amrum and Pellworm and the mainland of Schleswig Holstein as well as the nine other Halligen and three sand barrier islands. The inset shows the location of the area within the North Sea and its neighboring countries: GB = Great Britain, NL = The Netherlands, $D=$ Germany, $D K=$ Denmark, $\mathrm{S}=$ Sweden, $\mathrm{N}=$ Norway. 
The Halligen have a total area of approximately $23 \mathrm{~km}^{2}$ with an average elevation of only few decimetres above mean high water (MHW). They are inhabited by around 300 people. These low lying marsh areas are recognized as UNESCO World Heritage site, UNESCO biosphere reserve and flora-fauna habitat area of the European Union. Annually, millions of migratory birds use the vegetated marshlands for resting and breeding. In contrast to adjacent islands like Amrum and Föhr, which are consolidated by a core of Pleistocene glacial deposits, the Halligen consist solely of Holocene marshland sediments being prone to erosion during storm surge events. The most catastrophic storm surges in historical time, the so-called 1st and 2nd Grote Mandränke in 1362 and 1634, drowned thousands of people and caused enormous land losses in the North Frisian Wadden Sea (Reise 2005) what gave the Halligen their current isolated appearance as remnants of the former coherent marshlands (Ahrendt 2007). The authorities started to stabilize the Hallig margins around 1900 with revetments to stop the ongoing land loss. Between the first survey in 1713 and 1900 already 50-80 \% of the former Hallig marshland area was eroded (Quedens 1991). Small dyke constructions were built in 1914 on the two largest Halligen Hooge and Langeness (Müller and Fischer 1917). Further devastating storm surges like the event in 1962 forced the authorities to implement additional protection measures such as breakwater constructions which enclose the larger Halligen and protect parts of the smaller Halligen (see inset 1 in Figure 5-2). But even today, the Halligen are massively affected by extreme weather conditions, storm surges and by the ongoing regional MHW rise (Deicke et al. 2007). The latter forms the major threat in preservation of the Halligen for future generations. This is due to the fact that today's vertical accretion rates of three of the larger Halligen $(1.5 \pm 0.9 \mathrm{~mm} / \mathrm{a}$ for Hooge, $2.6 \pm 0.9 \mathrm{~mm} / \mathrm{a}$ for Nordstrandischmoor and $1.2 \pm 0.8$ $\mathrm{mm} / \mathrm{a}$ for Langeness; Schindler et al. 2014b) demonstrate that none of these marshlands keep pace with the local MHW rise of $5.0 \pm 0.3 \mathrm{~mm} / \mathrm{a}$ in the Hallig area (1951-2011; Schindler et al. 2014a) or the local mean sea level (MSL) rise in the inner North Sea of $4.59 \pm 1.82 \mathrm{~mm} / \mathrm{a}$ (19932009; Wahl et al. 2013).

The overall available suspended particulate matter (SPM) forms a key factor for the natural adaptability of the Halligen to rising MHW if SPM is deposited on the marshland during inundations. A study about the spatial and temporal distribution of SPM concentration and its controlling factors around Hallig Langeness has been performed by using an autonomously working turbidity measurement network (Hache et al. 2019). The results indicate sufficient SPM concentrations on the tidal flats around Langeness, which would allow for vertical accretion 
rates capable to compensate for local MHW rise if SPM reached and deposited efficiently on the Hallig marshland (Hache et al. 2020).

Here, we analyse seven strong inundations caused by storm surges in the measuring season from autumn 2019 to spring 2020. These events were sampled, analyzed and evaluated in the same way as eight moderate inundations that occurred in the period 2017-2019. For the latter, only $20 \%$ of the SPM concentration on the tidal flats is measured on the marshland, at the most. This concentration difference indicates a transport limitation of SPM from tidal flats towards the marsh surface. This has been related to effects of the present coastal protection measures (Hache et al. 2020). The present study evaluates these effects with respect to stronger inundation events and discusses implications for protection measures on the Halligen.

\subsection{Methods}

\subsubsection{Study area}

Hallig Langeness is the largest of ten Halligen with a total area of $9.24 \mathrm{~km}^{2}$ and is located in the tide-dominated North Frisian Wadden Sea off the coast of the federal state of SchleswigHolstein, Germany (Figure 5-1). The tide is semidiurnal with an average mean tidal range of 2.7 $m$ referenced relative to the German ordnance datum Normalhöhennull (NHN), measured at tide gauge Hilligenley (Figure 5-2) during the winter periods from October, 2017 to March, 2019. Such range is characteristic for mesotidal regimes according to Hayes (1979). The natural terrain height of Langeness is on average $1.53 \mathrm{~m}$ above NHN and thus only $0.11 \mathrm{~m}$ above MHW (Mean High Tide Water level). To protect Langeness against flow-induced erosion, approximately 50 spur dykes keep the channels of the open tidal flats at some distance to the Hallig edge (Figure 5-2). Coastal protection measures like dykes and revetments with an average height of $2.34 \mathrm{~m}$ above NHN (0.92 $\mathrm{m}$ above MHW) encompass the entire Hallig to protect Langeness from coastal erosion and minor inundations (Schindler and Willim 2015). These measures are especially relevant during the summer season when farmers cultivate their grassland and livestock while migratory birds rest and breed. Inundations of the marshland are common in the winter months and are usually caused by strong westerly winds superimposed 
on high astronomical tides. In case of an inundation, the 113 inhabitants (2018) protect themselves, their cattle and goods by living on man-made dwelling-mounts (on Langeness called "Warfen") which are on average $5.06 \pm 0.45 \mathrm{~m}$ above NHN (Figure 5-2). In order to withstand the rising water levels, the Warfen were heightened in 2000. Further increases are already implemented for the best possible protection of the inhabitants (Hofstede 2019). To reduce the wave energy on the marshland during inundations breakwater constructions with variable water permeability and varying heights from 0.85 to $3.27 \mathrm{~m}$ above NHN protect the edges of Langeness (inset 1, Figure 5-2). After an inundation, six tidal gates at the outlets of drainage channels ensure a fast dewatering of the marshland (inset 2, Figure 5-2).

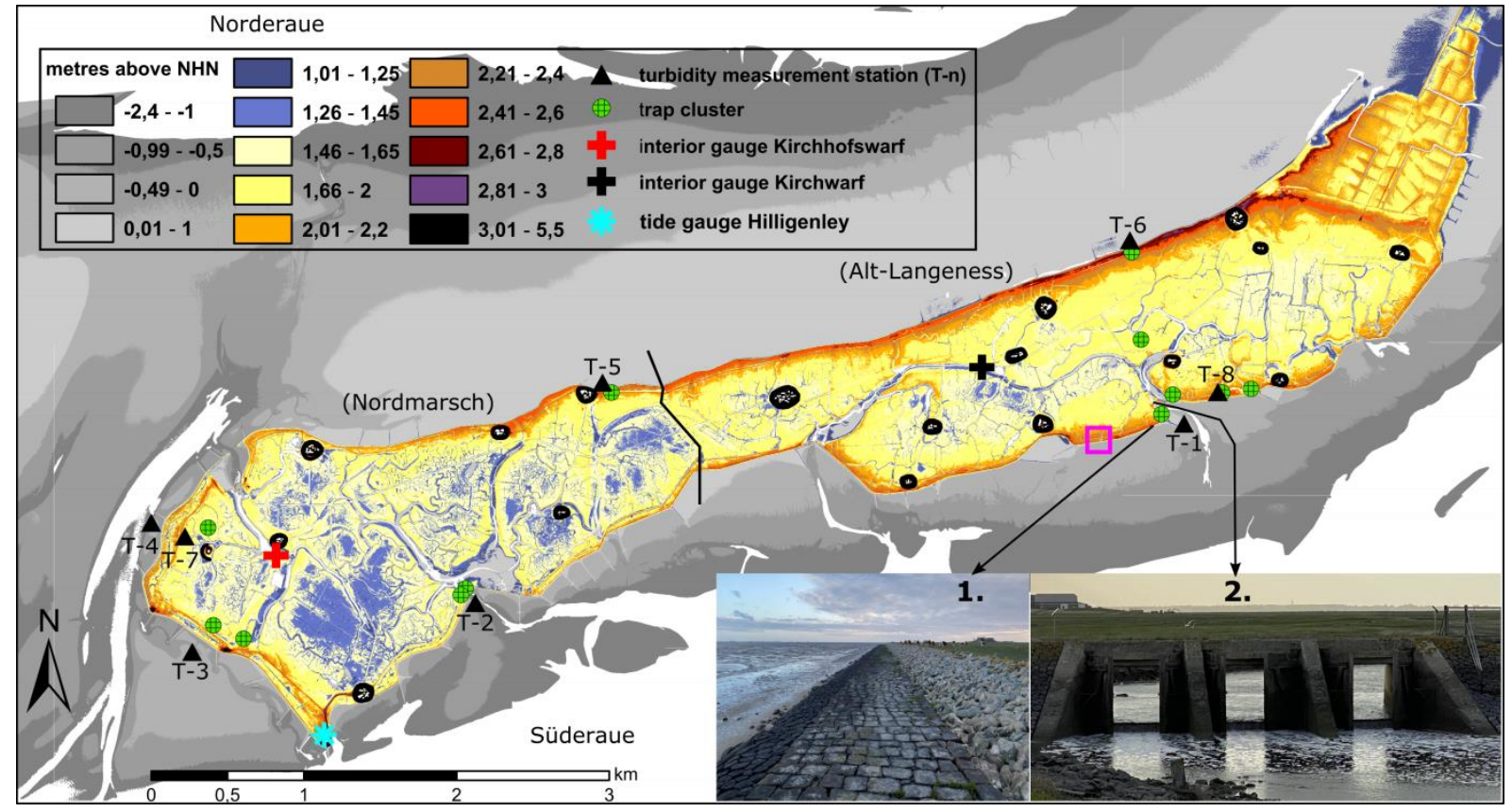

Figure 5-2: Digital ground model of Langeness (based on Laser scan DGM1 data from 2014, courtesy of LKN.SH) adjusted after Hache et al. (2020). The black line divides Langeness in its historical parts named Nordmarsch in the west and Alt-Langeness in the east. The white areas show depths $>2.4 \mathrm{~m}$ below NHN including the two main tidal channels Norderaue and Süderaue. The black roundish areas on the marsh surface are the dwelling-mounts. Inset 1 shows (from left to right) the tidal flat at low water next to the revetment and breakwater construction. Inset 2 shows a tidal gate at low water in the southeast of Langeness adjacent to turbidity measurement stations T-1 and T-8. The magenta box in the southeast shows the location where the photos were taken for Figure 5-8.

\subsubsection{Turbidity measurement network}

The autonomously working turbidity measurement network (Hache et al. 2019) includes eight measurement stations. Six were installed in the open tidal flat around Langeness (T-1 to T-6) and two (T-7, T-8) on Langeness itself (Figure 5-2). The installed optical backscatter turbidity 
sensor OBS-3+ by campbellC scientific (Campbell Scientific 2017) record the strength of backscattered light, which is a function of the number of particles in suspension and in this way a measure for the SPM concentration at the site. The measurement cycle starts as soon as the OBS is permanently submerged (one measurement per minute) and stops as soon as the water level drops below the OBS sensor unit. Therefore, stations T-1 to T-6 on the tidal flats measure SPM at almost every tide while the stations T-7 and T-8 on Langeness measure only when the Hallig is inundated. The measurement duration at the single stations thus depends on their elevation and the water height of individual tides.

Adjacent to each OBS sensor, a self-built sediment accumulator called SB for 'Base' is mounted (Design by IM+P engineering office Dr.-Ing. Manzenrieder und Partner GbR http://www.impol.de) to collect a sample of the sediments that are detected by the OBS. A second accumulator called ST for 'Top' is mounted approximately $1.2 \mathrm{~m}$ higher at each measurement station to investigate the vertical variability of SPM concentrations within the water column during individual inundations. The sediment accumulators have a capacity of approximately $350 \mathrm{~cm}^{3}$ and six horizontal inlets of $8 \mathrm{~mm}$ in diameter. The accumulators are closed at the top to minimize resuspension of sediment inside the accumulator. With an aspect ratio of 3.6 the accumulator fulfills the aspect ratio conditions recommended by Gardner (1980a, 1980b) to prevent resuspension at higher flow velocities. The sediments in the SB accumulators are used for calibrating the respective OBS sensors after each inundation to convert the OBS signal from millivolt to grams per liter. We therefore emptied the accumulators immediately before an inundation and sampled them as soon as the tidal flats were accessible again after the inundation to minimize a possible bias in the sediment accumulators caused by subsequent tides. The accumulator samples were wet sieved to filter out seagrass and shells and were subsequently stirred with an electric paint stirrer with sufficient water to get a homogeneous suspension. While stirring, aliquots were extracted from the suspension and transferred into a self-built calibration tank. A pump efficiently circulated the contained suspension inside the tank to ensure a homogeneous distribution. After each added aliquot the OBS response inside the tank was recorded for 60 seconds, averaged and assigned to the dried mass of solids. A more detailed description about the sediment accumulators and the OBS calibration procedure is given in Hache et al. (2019). 


\subsubsection{Sediment traps}

To quantify the sediment accumulation on the marshland after each inundation, we used a well-established combination of sediment traps consisting of one-liter LDPE bottles and PEsynthetic turf mats (Schindler et al. 2014b). The bottles measure $20 \mathrm{~cm}$ in height from the bottom of the bottle to the base of the screw cap and $9.5 \mathrm{~cm}$ in diameter. The opening of the bottle is $5 \mathrm{~cm}$ in diameter. The bottles were buried into the ground up to the screw cap and filled with water in order to prevent them from floating in case of an incipient inundation. Next to the bottles the turf mats were fixed to the ground with five nails. Each turf mat with $2.1 \mathrm{~cm}$ long grass like PE-blades has a surface area of $600 \mathrm{~cm}^{2}$. A combination of five of each trap type was installed crosswise with a distance of $5 \mathrm{~m}$ to each other in the field. This constellation is further called 'trap cluster'. The sediment accumulation data from the trap clusters are used to calculate annual vertical accretion rates according to Schindler et al. (2014b). A detailed description with illustrations about the whole setting of trap clusters, sediment accumulators and turbidity measurement stations is given in Hache et al. (2020).

\subsubsection{Grain size analysis}

Laser granulometry was applied to measure the grain size distribution (GSD) of all bottle trap and sediment accumulator samples. Therefore, an aliquot from the total sediment sample was extracted and treated for $30 \mathrm{~s}$ in a supersonic bath to fully disaggregate the fine-grained material. The measurements were carried out with a laser particle sizer LS13320 (Beckman\&Coulter) using the Fraunhofer optical model. Each sample was measured in three runs of 60 seconds within the range of 0.4 and $2000 \mu \mathrm{m}$. The runs were averaged.

\subsubsection{Inundation scenarios}

Strong wind events occur in particular during the winter months and the associated high water levels inundate Langeness around 10 times per year (averaged over the period 1961-2010; Schindler and Willim 2015). Depending on weather and tidal conditions, moderate inundations up to severe storm surges may occur. To distinguish between moderate inundation and storm surge events we refer to the classification of the German Federal Maritime and Hydrographic Agency (BSH). Accordingly, at the German North Sea coast a storm surge is defined by peak water level between 1.5 to 2.5 meters above $\mathrm{MHW}$. For the $\mathrm{MHW}$ water level, we refer to the nearby tide gauge Dagebüll $\left(54.731^{\circ} \mathrm{N}, 8.687^{\circ} \mathrm{E}\right)$ that is monitored by the Federal Waterways 
and Shipping Administration (WSA). Compared to the tide gauge Hilligenley on Langeness, tide gauge Dagebüll is more precise and the data availability is much higher. The MHW at Langeness is $1.42 \mathrm{~m}$ above NHN. A moderate inundation can occur at about 2.30-2.40 $\mathrm{m}$ above NHN (Schindler and Willim 2015) up to the peak water level threshold of $2.92 \mathrm{~m}$ above NHN. Storm surges exceed this water level threshold. The water level on Langeness is monitored at two interior tide gauges, one in the western part (Kirchhofswarf) and one in the eastern part (Kirchwarf) (Figure 5-2).

\subsubsection{SPM quantities}

The determined SPM concentrations within the water masses on the tidal flats around Langeness are considered as initially available for a potential transport of SPM towards the marshland during the flood phase of an inundation. Therefore, the SPM concentration measured on the tidal flats, averaged during the entire flood phase is defined as initial SPM concentration $\left(S P M_{i}\right)$. For the SPM concentration determined during rising water level on the marshland, we define $S P M_{r s}$. SPM $r$ is also an averaged concentration of all SPM measurements within the same flood phase as SPM $\mathrm{M}_{\mathrm{i}}$ SPM $\mathrm{M}_{\mathrm{rs}}$ allows to estimate the fraction of the $\mathrm{SPM}_{\mathrm{i}}$ that has overcome the protection measures and that is potentially available for SPM accumulation and vertical accretion on the marshland. Finally, we define $S P M_{f l}$, which is an averaged SPM concentration of all SPM measurements on the marshland during falling water levels at the ebb phase. The separation of marshland SPM concentration for flood and ebb phase appears necessary due to the strongly asymmetrical gauge data for storm surges compared to moderate inundations (Figure 5-3). The $\mathrm{SPM}_{\mathrm{fl}}$ values are relevant for estimation of the fraction of $\mathrm{SPM}_{\mathrm{rs}}$ that may get lost with the draining water flow. To enable a longer time series of $\mathrm{SPM}_{\mathrm{fl}}$ at the draining water during the ebb phase of storm surges, we installed an additional turbidity measurement station (T-G). Station T-G is installed within a drainage channel directly landward behind a tidal gate, adjacent to station T-1 and T-8 (see 2. inset in Figure 5-2). Unlike the marshland stations T-1 and T-8, station T-G collected data during the whole tide due to low installation altitude. Unfortunately, the collected data of station T-G from four out of five storm surges had to be discarded because seagrass was wrapped around the OBS sensor. 


\subsection{Results}

\subsubsection{Inundation scenarios}

At the beginning of an inundation, the water level oversteps the mean height of the water impermeable coastal protection structures at $2.34 \mathrm{~m}$ above NHN. This is indicated by the water level of the tide gauge Hilligenley crossing the gray dashed lines in Figure 5-3. First, the drainage channels on Langeness fill up with water. When the mean ground level of Langeness with 1.53 above NHN is reached (dashed green lines in Figure 5-3), the water in the creeks and channel overtops the banks. The subsequent onset of the water flow over the entire marshland leads to a temporary decrease of the interior gauge level slopes. As soon as both interior tide gauge levels reach the mean height of the water impermeable coastal protection structures, the whole Hallig is completely inundated and only the dwelling-mounts and higher parts of the coastal protection structures stand above the water. The tidal gates mainly control the drainage water flow on the marsh surface (e.g. see inset 2, Figure 5-2). Consequently, the longer lasting ebb phases on the Hallig compared to the tidal flat lead to asymmetric interior gauge level curves at all inundations (Figure 5-3). 


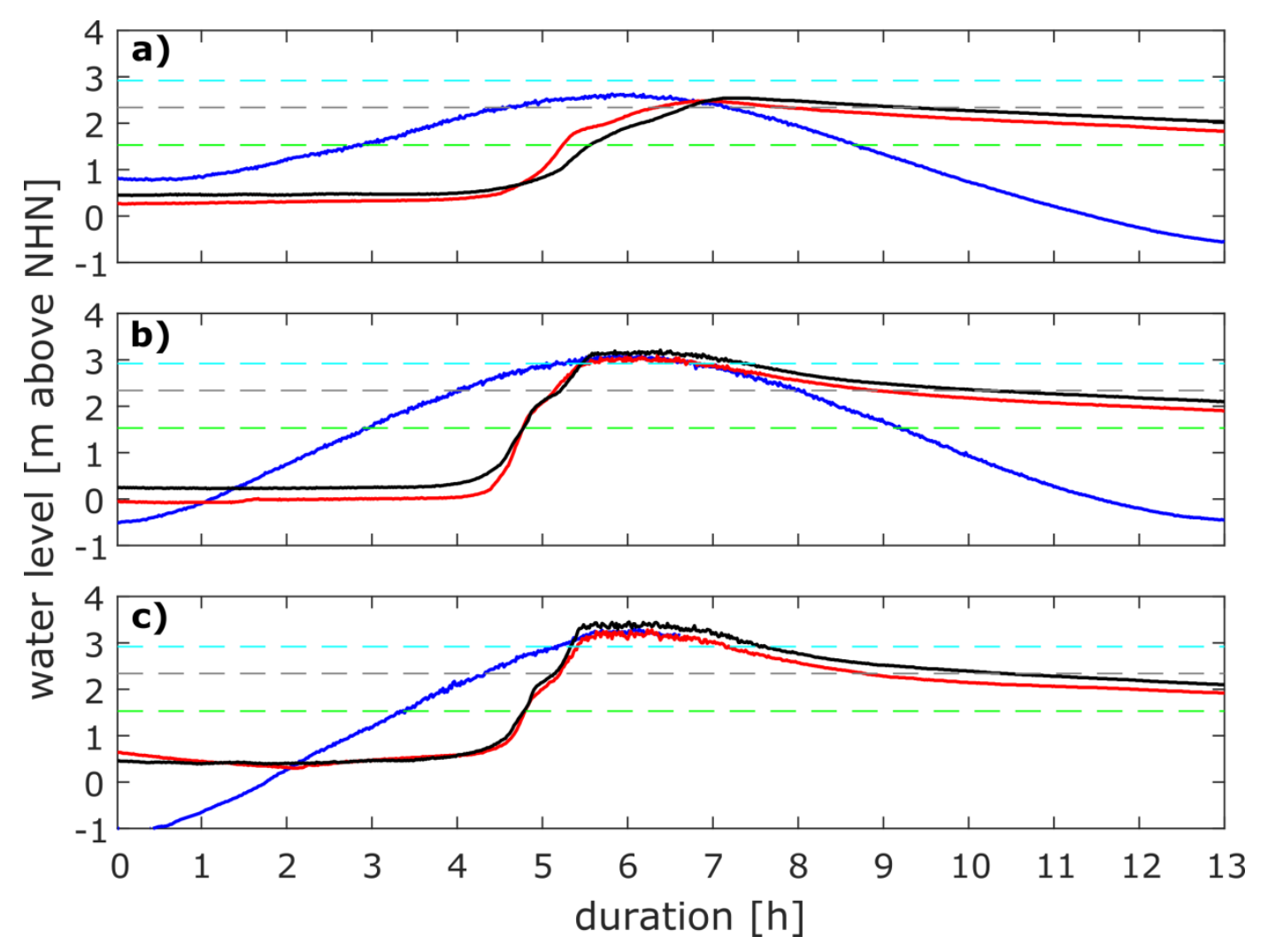

Figure 5-3: Gauge level data from the tide gauge Hilligenley (blue), the interior tide gauges Kichhofswarf (red) and Kirchwarf (black); a) example of a moderate inundation that occurred on March 05, 2019, b) storm surge SF2 at January 15, 2020 and c) storm surge SF1 at December 15, 2019. Dashed lines show the mean ground level height of $1.53 \mathrm{~m}$ above NHN of Langeness (green), mean height of the water impermeable coastal protection structures of $2.34 \mathrm{~m}$ above NHN (grey; Schindler and Willim 2015) and the reference height for storm surge water levels of $2.92 \mathrm{~m}$ above NHN (cyan). At storm surge SF1 no data was recorded by tide gauge Hilligenley during the ebb phase due to technical issues (c).

At a moderate inundation, it takes $115 \pm 14$ minutes until the Hallig is completely inundated (Figure 5-3a). With a peak water level of $2.51 \pm 0.04 \mathrm{~m}$ above NHN, the moderate inundation on March 5, 2019 remains below the storm surge threshold of $2.92 \mathrm{~m}$ above NHN indicated by the blue line in Figure 5-3a. With a peak water level of $3.16 \pm 0.08 \mathrm{~m}$ above NHN (Table 5-1), the inundation (SF2) on January 15, 2020 exceeded the storm surge threshold of $2.92 \mathrm{~m}$ above NHN (Figure 5-3b). At this storm surge, it took $70 \pm 3$ minutes until Langeness was completely inundated. The storm surge (SF1) on December 15, 2019 reached a peak water level of $3.37 \pm$ $0.12 \mathrm{~m}$ above NHN along with the highest tidal range of $4.31 \mathrm{~m}$ above NHN of all measured events (Table 5-1). Langeness was inundated fastest at SF1 (52 \pm 2 minutes). Note that the third storm surge (SF3) on February 10, 2020 took $65 \pm 8$ minutes to inundate the entire marshland during the first tide (SF3a). In the subsequent four tides (SF3b - SF3e), the water could not drain completely throughout the tidal gates before the Hallig was inundated again. 
Table 5-1: Parameters of seven storm surge induced inundations.

\begin{tabular}{|c|c|c|c|c|c|c|c|c|c|}
\hline inund & date & peak & tidal & wind & wind & significa & wind & wind & significa \\
\hline ation & & water & rang & speed & directio & nt wave & speed & directio & nt wave \\
\hline \multirow[t]{4}{*}{ event } & & level & e & (flood & n (flood & height & (ebb & n (ebb & height \\
\hline & & [m & {$[\mathrm{m}]^{*}$} & phase) & phase) & (flood & phase & phase) & (ebb \\
\hline & & above & $*$ & {$[\mathrm{~m} / \mathrm{s}]^{* *}$} & [degree & phase) & ) & [degree & phase) \\
\hline & & NHN]* & & $*$ & ] & {$[\mathrm{m}]^{* * * *}$} & {$[\mathrm{~m} / \mathrm{s}]$} & ] & [m] \\
\hline \multirow[t]{2}{*}{ SF1 } & 15.12 .2019 & $3.37 \pm$ & 4.31 & $20.4 \pm$ & $246 \pm 9$ & $0.9 \pm$ & $12.3 \pm$ & $244 \pm$ & $0.64 \pm$ \\
\hline & & 0.12 & & 2.1 & & 0.23 & 2.6 & 12 & 0.22 \\
\hline \multirow[t]{2}{*}{ SF2 } & 15.01 .2020 & $3.16 \pm$ & 3.61 & $16.1 \pm$ & $201 \pm 3$ & $0.67 \pm$ & $15.1 \pm$ & $201 \pm 3$ & $0.5 \pm$ \\
\hline & & 0.08 & & 0.8 & & 0.09 & 0.8 & & 0.07 \\
\hline \multirow[t]{2}{*}{ SF3a } & 10.02 .2020 & $3.65 \pm$ & 3.18 & $16.7 \pm$ & $267 \pm 6$ & $0.93 \pm$ & $15.4 \pm$ & $246 \pm$ & $0.88 \pm$ \\
\hline & & 0.05 & & 1.6 & & 0.2 & 2.3 & 16 & 0.2 \\
\hline \multirow[t]{2}{*}{ SF3b } & 11.02 .2020 & $2.98 \pm$ & 2.2 & $15 \pm 1.4$ & $258 \pm$ & $0.79 \pm$ & $16.4 \pm$ & $261 \pm 7$ & $0.99 \pm$ \\
\hline & & 0.1 & & & 15 & 0.1 & 1.6 & & 0.12 \\
\hline \multirow[t]{2}{*}{ SF3c } & 11.02 .2020 & $3.24 \pm$ & 2.52 & $16.4 \pm$ & $267 \pm 6$ & $0.98 \pm$ & $16.7 \pm$ & $265 \pm 6$ & $1.07 \pm$ \\
\hline & & 0.11 & & 1.3 & & 0.14 & 1.3 & & 0.12 \\
\hline \multirow[t]{2}{*}{ SF3d } & 12.02 .2020 & $3.79 \pm$ & 3.11 & $16.9 \pm$ & $266 \pm 8$ & $0.96 \pm$ & $16.3 \pm$ & $270 \pm 9$ & $1.08 \pm$ \\
\hline & & 0.06 & & 1.8 & & 0.15 & 1.4 & & 0.05 \\
\hline \multirow[t]{2}{*}{ SF3e } & 12.02 .2020 & $2.94 \pm$ & 2.55 & $15.5 \pm$ & $269 \pm 5$ & $0.95 \pm$ & $13.7 \pm$ & $269 \pm 4$ & $0.92 \pm$ \\
\hline & & 0.09 & & 1.4 & & 0.11 & 1.3 & & 0.06 \\
\hline
\end{tabular}

*Average peak water levels from both interior tide gauges Kirchhofswarf and Kirchwarf (Figure 5-2).

${ }^{* *}$ The tidal ranges were recorded at the tide gauge Hilligenley

***Ten minutes averaged mean wind speeds and directions from the weather station

Strucklahnungshörn (located $22 \mathrm{~km}$ southeast of the tide gauge Hilligenley)

$* * * *$ Significant wave height recorded by the sea state buoy Süderaue (located in the southern tidal channel Süderaue at about $2.5 \mathrm{~km}$ distance to the tide gauge Hilligenley), each for the flood and ebb phase. 


\subsubsection{SPM quantities}

The relation between SPM concentration on the tidal flat $\left(S P M_{i}\right)$ and the SPM concentration on the marshland during rising sea level $\left(\mathrm{SPM}_{\mathrm{rs}}\right)$ for the new storm surge data from the winter season $2019 / 20$ is compared to data from moderate inundations from a previous study (Hache et al. 2020). We focus on two stations on the tidal flat (T-4 in the west and T-1 in the southeast of Langeness), which are directly related to nearby stations on the marshland (T-7 and T-8, respectively; Figure 5-2).

In general, $\mathrm{SPM}_{\mathrm{rs}}$ increases with increasing $\mathrm{SPM}_{\mathrm{i}}$ at both sites (Figure 5-4). In comparison to moderate inundations, $\mathrm{SPM}_{\mathrm{i}}$ during storm surges increases by a factor of 3.1 in the east (T-1) and by a factor of 3.5 in the west of Langeness (T-4). SPM $\mathrm{rs}_{\mathrm{s}}$ also increases on average over all analyzed storm surges from $9 \%$ of $\mathrm{SPM}_{i}$ for moderate inundations to $28 \%$ at storm surge conditions in the west (T-7) and on average from $20 \%$ to $49 \%$ in the east (T-8). During the storm surge event SF1, SPM $\mathrm{i}_{\mathrm{i}}$ at both sites was highest and associated with the highest SPM $\mathrm{M}_{\mathrm{rs}}(73$ $\%$ of $\mathrm{SPM}_{\mathrm{i}}$ ) in the eastern location (Figure 5-4).

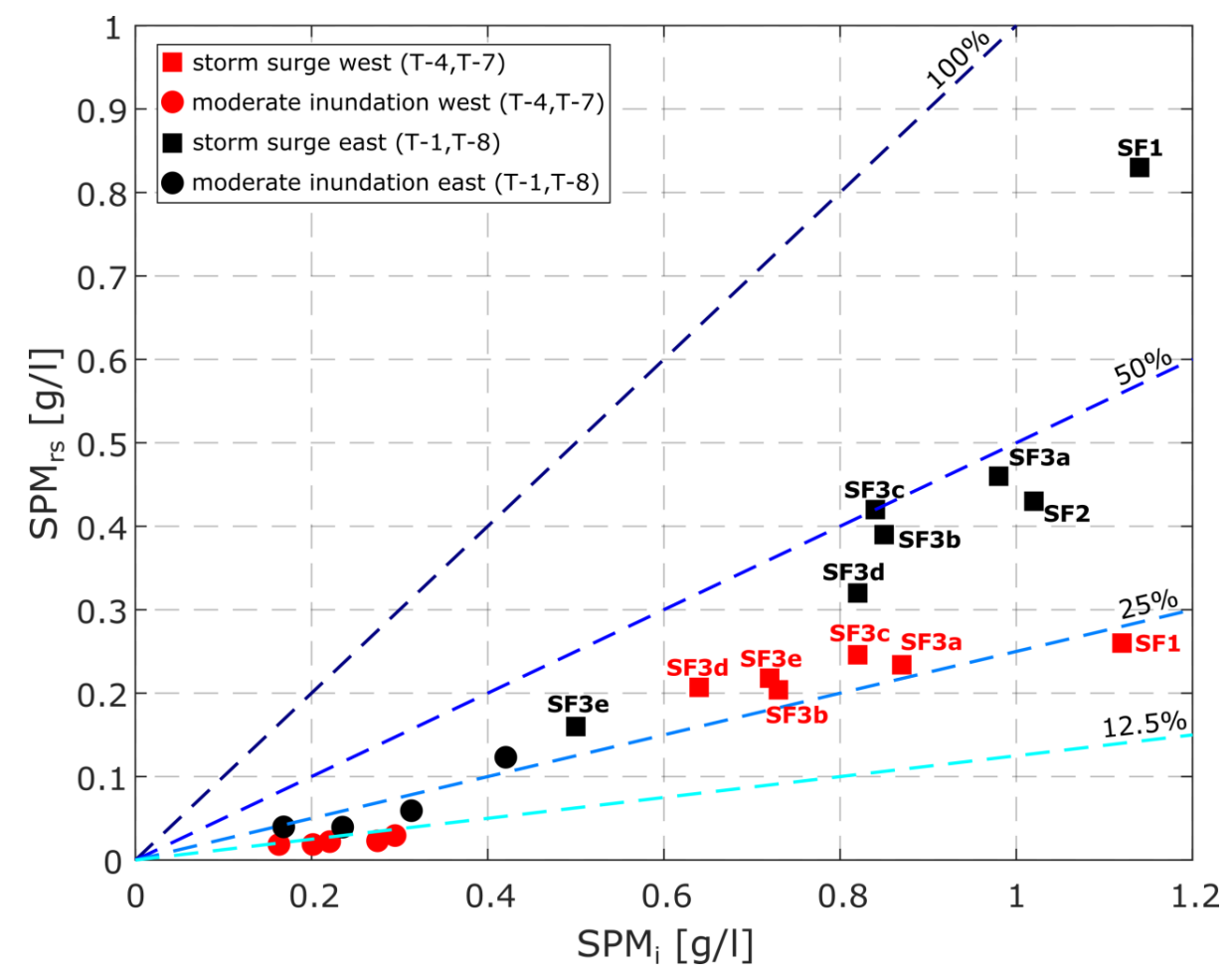

Figure 5-4: Comparison of $S P M_{i}$ on the tidal flats $(T-4, T-1)$ with $S P M_{r s}$ on the marshland at rising water level (T-7, T-8). In addition to data from seven storm surge inundations (squares) SPM values of individual moderate inundations recorded by Hache et al. (2020) are shown (circles). The $\mathrm{SPM}_{\mathrm{i}}$ at station T-4 are compared with SPM $\mathrm{S}_{\mathrm{rs}}$ at station T-7 (west) and SPM at T-1 with SPM $\mathrm{S}_{\mathrm{rs}}$ at T-8 (east). Individual storm surge events are labelled according to Table 5-1. The dashed lines show, for orientation, percentages of $\mathrm{SPM}_{\mathrm{i}}$ attained by $\mathrm{SPM}_{\mathrm{rs}}$. 
The storm surge data with longer measurement durations allows for assessing the sediment accumulation on the Hallig by comparing individual SPM concentrations between the flood and ebb phases. At all stations, individual SPM concentrations reach higher peak values during the flood phase than during the ebb phase. The higher elevated station T-8, however, shows a stronger decrease in SPM concentrations during the ebb phase of the first tide (SF3c) in Figure 5-5 than station T-G. In the following, the SPM concentrations during the drainage water flow at station T-G remain higher in the drainage channel than at the adjacent station T-8 on the marshland (black box in Figure 5-5). In the subsequent inundation SF3d, the difference in SPM concentrations of station T-8 and T-G between the flood and ebb phase remain rather low.

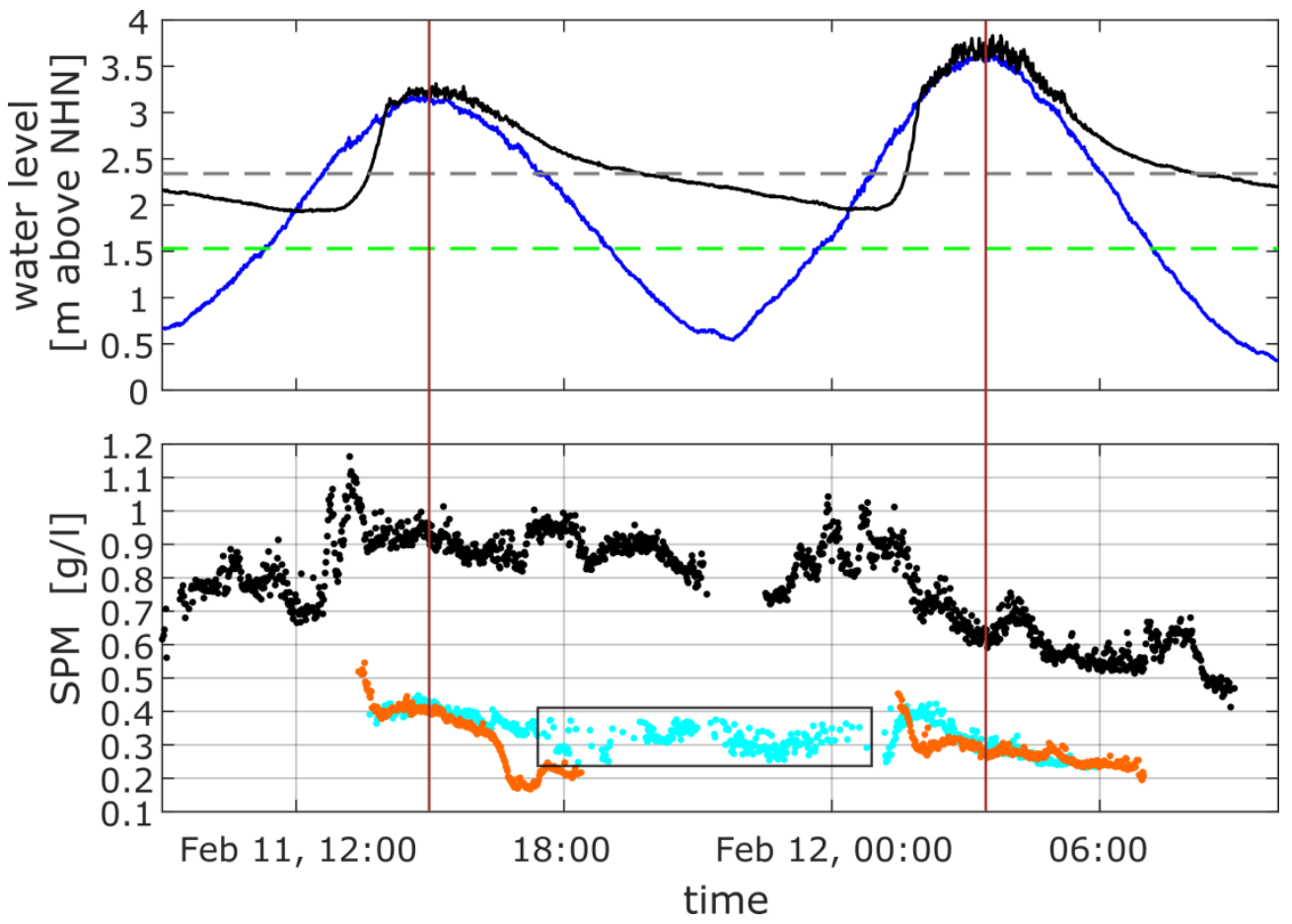

Figure 5-5: Water level data (upper graph) of tide gauge Hilligenley (blue) and interior tide gauge Kirchwarf (black). The lower graph shows individual SPM concentrations (not averaged) from three stations T-1 (black), T-8 (orange) and T-G (cyan) during the two storm surge induced inundations SF3c and SF3d on February 11 and 12, 2020 (Table 5-1). The black box highlights the SPM concentrations at station T-G during the drainage water flow through the tidal gate. The red vertical lines resemble the separation into flood/ebb phase.

In general, we observed that the differences between $\mathrm{SPM}_{\mathrm{rs}}$ and $\mathrm{SPM}_{\mathrm{fl}}$ of all measured storm surges remain rather small at each station on the marshland (later shown in Figure 5-6). In contrary, the measured sediment accumulation showed rather high values. We think that this discrepancy between rather high SPM concentrations during ebb phase and high sediment accumulation values is due to a calibration issue of the optical backscatter sensors caused by the strong grain size dependency of the OBS signal. Therefore, we decided to distinguish 
between SPM concentrations during the ebb phase generated with a 'coarse-calibration' and a 'fine-calibration' by using large and small grain sizes in the calibration procedure, repectively. For the coarse-calibrated $\mathrm{SPM}_{\mathrm{fl}}$, sediment accumulator samples from individual storm surges were used. The individual storm surge calibration samples show median grain sizes of $27 \mu \mathrm{m}$ to $55 \mu \mathrm{m}$ for station T-7, $42 \mu \mathrm{m}$ to $90 \mu \mathrm{m}$ for station T-8 and $81 \mu \mathrm{m}$ for station T-G (Table 5-2). Note that the sediment accumulator calibration sample from event SF3 at station T-7 contains the suspended sediment input of all five storm surge-induced inundations because it was not possible to reach the station between the individual tides. At station T-8, it was possible to reach the sediment accumulator after every two tides that inundated the marshland (Table 5-2). The fine-calibrated $\mathrm{SPM}_{\mathrm{fl}}$ was generated with sediment accumulator samples from a moderate inundation. The median grain sizes of the moderate inundation are $20 \mu \mathrm{m}$ for station T-7, $26 \mu \mathrm{m}$ for station T-8 and $26 \mu \mathrm{m}$ for station T-G (Table 5-2).

Table 5-2: Median grain sizes of sediment accumulator samples from individual storm surge induced inundations and one moderate inundation that is used for the individual OBS sensor calibration.

\begin{tabular}{cccccc}
\hline $\begin{array}{c}\text { Inundation } \\
\text { events }\end{array}$ & T-4 $[\mu \mathrm{m}]$ & $\mathrm{T}-7[\mu \mathrm{m}]$ & $\mathrm{T}-1[\mu \mathrm{m}]$ & $\mathrm{T}-8[\mu \mathrm{m}]$ & $\mathrm{T}-\mathrm{G}[\mu \mathrm{m}]$ \\
\hline SF1 & 154 & 39 & 118 & 90 & - \\
SF2* & - & 27 & 107 & 42 & - \\
SF3a+b** & 136 & 55 & 117 & 86 & 81 \\
SF3c+d & 136 & 55 & 124 & 85 & 81 \\
SF3e & 136 & 55 & 109 & 39 & 81 \\
moderate & - & 20 & - & 26 & 26
\end{tabular}

*At storm surge SF2, it was not possible to reach the station T-4 immediately before the storm surge. ${ }^{* *}$ At storm surge SF3, the sediment accumulator at station T-1 and T-8 were sampled, each after two tides that inundated the marshland. At station T-4, T-7 and T-gate it was only possible to reach the stations after the entire five storm surge cycles.

The values of coarse- and fine-calibrated $\mathrm{SPM}_{\mathrm{fl}}$ in comparison to $\mathrm{SPM}_{\mathrm{rs}}$ are shown in Figure 5-6. Next to the tidal gate with some safety distance to the rapidly draining water masses, we took suspension samples during the ebb phase of the storm surges SF3b and SF3d to validate the $\mathrm{SPM}_{\mathrm{fl}}$ calibrations. After filtration, drying and weighing dry mass of suspension samples 
indicated a $\mathrm{SMP}_{\mathrm{fl}}$ of 0.028 to $0.053 \mathrm{~g} / \mathrm{l}$ and were assigned to the $\mathrm{SPM}_{\mathrm{rs}}$ values of station $\mathrm{T}-8$ due to its adjacent position (Figure 5-6).

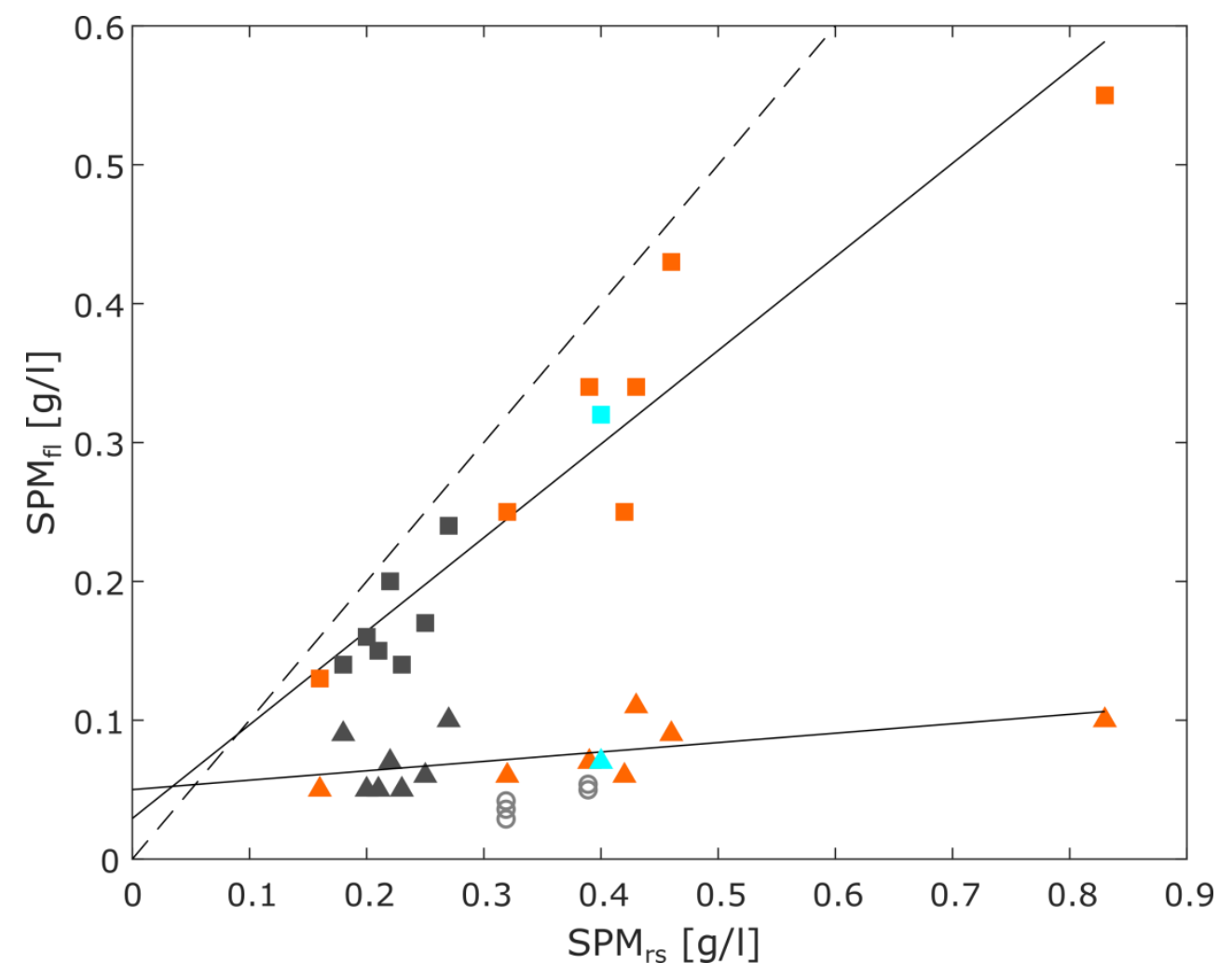

Figure 5-6: $\mathrm{SPM}_{\mathrm{rs}}$ versus $\mathrm{SPM}_{\mathrm{fl}}$ for seven storm surge induced inundations. The values at T-7 (dark grey), T-8 (orange) and T-G (cyan) from two different OBS calibrations, coarse grain size calibrated SPM (squares) and the fine grain size-calibrated SPM (triangles). The linear fits refer to the SPM concentrations of station T-7 and T-8. The five circles (light grey) show the dry mass concentration in suspension samples taken as reference. The dashed line indicates the 1:1 ratio between SPM $_{\mathrm{rs}}$ and $S P M_{\mathrm{fl}} \cdot$

In general, the coarse- and fine-calibrated $\mathrm{SPM}_{\mathrm{fl}}$ increase with increasing $\mathrm{SPM}_{\mathrm{rs}}$ at both sites, highlighted by both linear fits in Figure 5-6. The coarse-calibrated $\mathrm{SPM}_{\mathrm{fl}}$ is close to the 1:1 ratio line (meaning that only minor accumulation occurred on the marshland) and the fine-calibrated $\mathrm{SPM}_{\mathrm{fl}}$ is close to the dry mass concentrations of in situ taken suspension samples and therefore seems more realistic. 


\subsubsection{Variation in grain size distribution}

We compared the GSD of sediment accumulator samples at station T-1 on the tidal flat with the GSD of the sediment samples from two adjacent sediment trap clusters. These trap clusters are located on the marshland behind breakwaters (see inset 1, Figure 5-2) of different heights. The breakwater at cluster $1 \mathrm{H}$ with $2.86 \pm 0.14 \mathrm{~m}$ above NHN is on average $0.47 \mathrm{~m}$ higher than the breakwater at cluster $1 \mathrm{~L}$ with $2.39 \pm 0.04 \mathrm{~m}$ above NHN (elevation data from GNSS topographical survey of the LKN.SH). For the grain size comparison, we use data from three individual inundations (Figure 5-7).
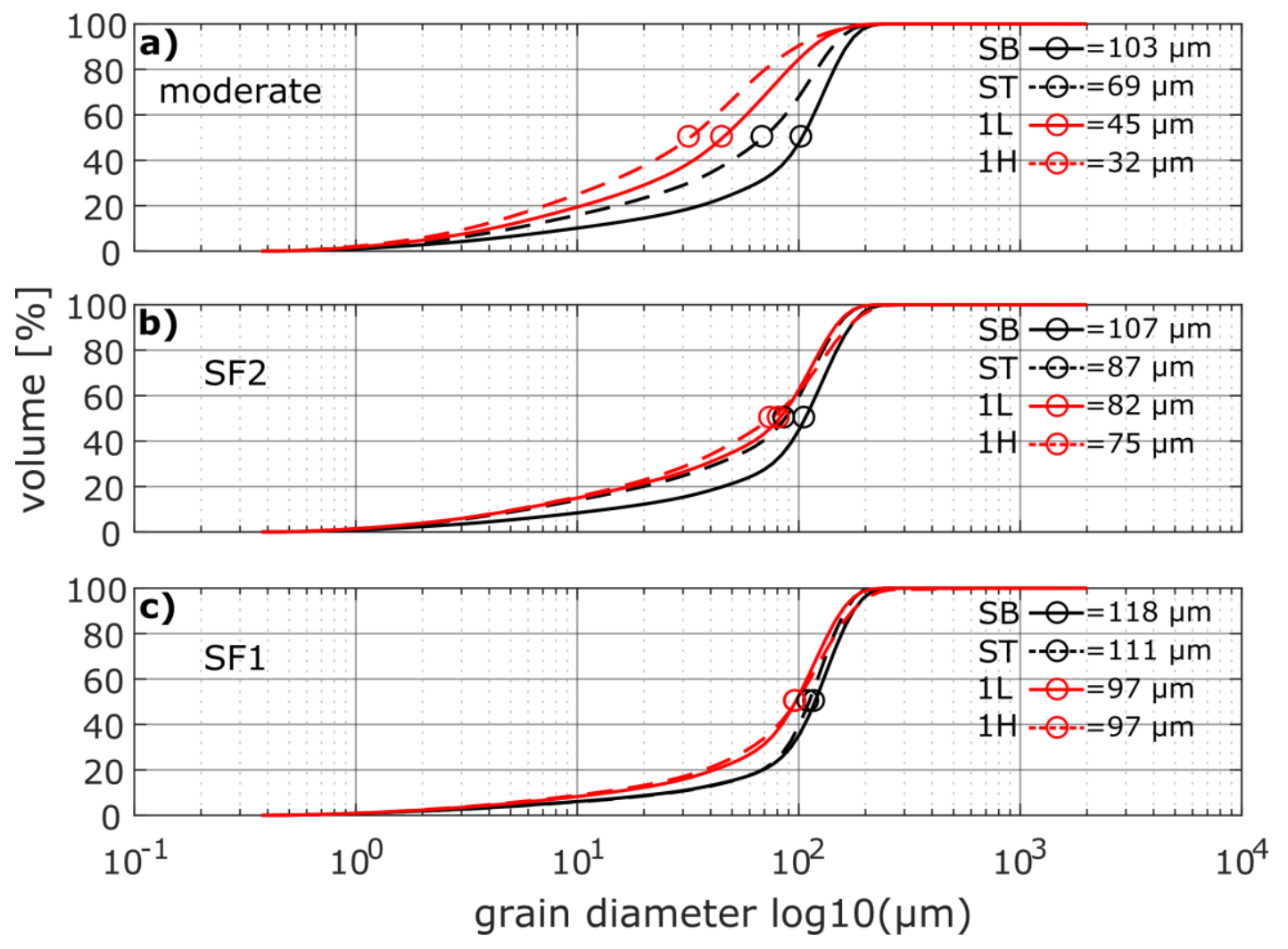

Figure 5-7: Grain size distribution (0.4 to $2000 \mu \mathrm{m}$ ) of the sediment accumulator samples SB and ST at station T-1 and the adjacent sediment trap samples $1 \mathrm{~L}$ (lower breakwater) and $1 \mathrm{H}$ (higher breakwater) from a moderate inundation (a), storm surge SF2 (b) and storm surge SF1 (c) (Increasing peak water level). The respective median is displayed as circle. The respective samples were collected during one single tidal cycle. The associated tide gauge levels are shown in Figure 5-3 in the same order from (a) to (c).

The differences between the GSD on the tidal flat and on the marshland are evident at all moderate inundations (Figure 5-7a). The sediment accumulator SB sample shows the coarsest and the sample of the trap cluster behind the higher breakwater $(1 \mathrm{H})$ the finest GSD (median $103 \mu \mathrm{m}$ vs. $32 \mu \mathrm{m}$, respectively). At storm surge SF2, the GSD becomes overall coarser while the decreasing trend from sediment accumulator SB sample to the trap cluster $1 \mathrm{H}$ sample is 
preserved but less pronounced (Figure 5-7b). At storm surge SF1, the GSD is the coarsest of all measured events, on the tidal flat as well as on the marshland behind different breakwater heights (Figure 5-7c) while the contrast between accumulator samples and trap cluster is minimized. The average median grain size of the trap cluster samples $1 \mathrm{H}$ and $1 \mathrm{~L}$ increase from $39 \pm 10 \mu \mathrm{m}$ for eight moderate inundations to $86 \pm 10 \mu \mathrm{m}$ for the seven storm surges.

\subsubsection{Sediment accumulation}

Considering sediment trap samples (bottles) next to T-7 and T-8 we compare SPM $\mathrm{M}_{\mathrm{rs}}$ with the sediment accumulation of moderate inundations (detailed in Hache et al. 2020) and storm surges (Table 5-3).

In general, sediment accumulation positively correlates with increasing $\mathrm{SPM}_{\mathrm{rs}}$ at both sites (Table 5-3). Sediment accumulation in the west is generally lower than in the east. During storm surges, the difference in sediment accumulation is decreasing. The sediment accumulation at T7 is only $8 \%$ of T-8 at moderate inundations and increases to $25 \%$ at storm surge conditions. Over all, the average sediment accumulation determined with all trap clusters on the marshland is 8.9 fold higher at storm surges compared to moderate inundations (Table 5-3). The storm surge SF1 that already showed the highest SPM $\mathrm{M}_{i}$ and the highest $\mathrm{SPM}_{\mathrm{rs}}$ (Figure 5-4) also shows the highest sediment accumulation within a single tide that inundates Langeness. The storm surge SF2 shows a lower sediment accumulation along with a lower SPM rs. The last tide SF3e shows the lowest sediment accumulation within the storm surge SF3 (Table 5-3). 
Table 5-3: Sediment accumulation from sediment traps (bottles) and SPMrs as average (av) of all measured moderate inundations and storm surges at station T-7, T-8 and from all 12 sediment trap fields (bottles) on the marshland.

\begin{tabular}{|c|c|c|c|c|c|}
\hline & $\begin{array}{c}\mathrm{T}-7 \\
\text { accumulation } \\
{\left[\mathrm{g} / \mathrm{m}^{2}\right]^{* *}}\end{array}$ & $\begin{array}{c}\text { T-7 SPM }{ }_{\mathrm{rs}} \\
{[\mathrm{g} / \mathrm{I}]}\end{array}$ & $\begin{array}{c}\mathrm{T}-8 \\
\text { accumulation } \\
{\left[\mathrm{g} / \mathrm{m}^{2}\right]}\end{array}$ & T-8 SPM rs $[\mathrm{g} / \mathrm{I}]$ & $\begin{array}{c}\text { All trap } \\
\text { clusters } \\
\text { accumulation } \\
{\left[\mathrm{g} / \mathrm{m}^{2}\right]^{* * *}}\end{array}$ \\
\hline $\begin{array}{l}\text { av moderate } \\
\text { inundation }\end{array}$ & $15 \pm 17.7$ & 0.02 & $187.2 \pm 95.8$ & 0.06 & $144 \pm 49$ \\
\hline $\begin{array}{c}\text { av storm } \\
\text { surge }\end{array}$ & $222.3 \pm 76.3$ & $0.22 \pm 0.03$ & $878.9 \pm 602.3$ & $0.43 \pm 0.2$ & $1279 \pm 778$ \\
\hline SF1 & 358.6 & 0.26 & 1959 & 0.83 & 1154.2 \\
\hline SF2 & 181.1 & 0.18 & 828.1 & 0.43 & 572 \\
\hline$S F 3 a+b$ & - & $0.44 *$ & 1310.6 & 0.84 & - \\
\hline$S F 3 c+d$ & - & 0.45 & 1722.6 & 0.74 & - \\
\hline SF3e & - & 0.22 & 216.7 & 0.16 & - \\
\hline sum SF3 & 953.4 & 1.11 & 3249.9 & 1.74 & 2112.2 \\
\hline $\begin{array}{l}\text { *Values of the in } \\
\text { SPM } \\
\text { r* of more th } \\
\text { two tides that in } \\
\text { cluster after the } \\
{ }^{* * *} \text { The sedimen } \\
\text { marshland. Sedir } \\
\text { of SF3a to SF3e. }\end{array}$ & $\begin{array}{l}\text { dividual samplec } \\
\text { an one tide are } \\
\text { ons, it was nece } \\
\text { undated the mar } \\
\text { entire five storm } \\
\text { accumulation r } \\
\text { nent accumulati }\end{array}$ & $\begin{array}{l}\text { tervals resu } \\
\text { ned in bold. } \\
\text { ary to sample } \\
\text { land. At stati } \\
\text { urge induced } \\
\text { s are averag } \\
\text { of storm sur }\end{array}$ & $\begin{array}{l}\text { from a summa } \\
\text { sediment trap } \\
-7 \text { it was only } p \\
\text { dations. } \\
\text { lues from all se } \\
\text { F3e shows the }\end{array}$ & $\begin{array}{l}\text { n of sediment a } \\
\text { station T-8 at } \\
\text { sible to reach th } \\
\text { nent traps of ty } \\
\text { nulative sedime }\end{array}$ & $\begin{array}{l}\text { cumulation and } \\
\text { adjacent trap } \\
\text { adteach after } \\
\text { t accumulation }\end{array}$ \\
\hline
\end{tabular}




\subsubsection{Accretion rates}

In each of the last four winter seasons from 2016/17 to 2019/20, we calculated average vertical accretion rates from all trap clusters (Table 5-4, Figure 5-2) according to Schindler et al. (2014b). In contrast to the winter season 2016/17, the winter seasons 2017/18 and 2018/19 showed only moderate inundations with lower inundation heights. This resulted in a low annual average accretion rate of around $1 \mathrm{~mm} / \mathrm{a}$ with an average accretion rate of $0.3 \mathrm{~mm}(2017 / 18)$ and $0.2 \mathrm{~mm}$ (2018/19) per inundation. The cumulative sediment accumulation of seven storm surges SF1 to SF3e in 2019/20 (Table 5-3) resulted in an average vertical accretion of $5.9 \pm 3.8$ $\mathrm{mm}$. Hence, the average accretion rate per inundation during a single storm surge amounts to $0.84 \mathrm{~mm}$. To calculate the annual vertical accretion rate for the winter season 2019/20, we have to take into account additional six inundations that occurred between February 17 and March 13, 2020. These six additional inundation events were only cumulatively sampled with all trap clusters. Three of the additional six events were moderate inundations with an average inundation height of $2.6 \pm 0.2 \mathrm{~m}$ above NHN. The remaining three events were storm surges with an average inundation height of $3.0 \mathrm{~m}$ above NHN. Based on the sediment accumulation, determined with the bottle traps, all 13 inundations result in an average annual vertical accretion rate of $8.4 \pm 5.1 \mathrm{~mm}$. Combining both trap types from the trap clusters leads to an average annual vertical accretion rate of $7.4 \pm 4.6 \mathrm{~mm}$ in 2019/20 (Table 5-4). This is distinctly higher than the average vertical accretion rate of $2.8 \pm 2 \mathrm{~mm}$ for the last four years (2016-2020; Table 5-4). 
Table 5-4: Vertical marshland accretion rates $\Delta \mathrm{H}$ of four sampled winter seasons including minimum and maximum values $\Delta \mathrm{H}$ and the average inundation height.

\begin{tabular}{|c|c|c|c|c|c|}
\hline winter season & $\begin{array}{c}\text { used } \\
\text { sediment } \\
\text { traps }\end{array}$ & $\begin{array}{c}\text { number of } \\
\text { sampled } \\
\text { inundations }\end{array}$ & $\Delta \mathrm{H}[\mathrm{mm} / \mathrm{a}]$ & $\begin{array}{c}\Delta \mathrm{H} \mathrm{min} / \mathrm{max} \\
{[\mathrm{mm} / \mathrm{a}]}\end{array}$ & $\begin{array}{c}\text { average } \\
\text { inundation } \\
\text { height [m } \\
\text { above } \\
\text { NHN]**** }\end{array}$ \\
\hline $16 / 17$ & $\begin{array}{c}\text { bottle }+ \text { turf } \\
\text { mat }\end{array}$ & 6 & $1.9 \pm 1.9$ & $0.4 / 6.5$ & $2.9 \pm 0.4$ \\
\hline $17 / 18$ & $\begin{array}{c}\text { bottle }+ \text { turf } \\
\text { mat }\end{array}$ & 3 & $0.9 \pm 0.5$ & $0.1 / 1.8$ & $2.6 \pm 0.1$ \\
\hline $18 / 19$ & $\begin{array}{c}\text { bottle }+ \text { turf } \\
\text { mat }\end{array}$ & 5 & $1.0 \pm 0.9$ & $0.1 / 2.7$ & $2.5 \pm 0.2$ \\
\hline 19/20_T* & bottle & 7 & $5.9 \pm 3.8$ & $1.7 / 12.3$ & $3.3 \pm 0.3$ \\
\hline $19 / 20 * *$ & bottle & 13 & $8.4 \pm 5.1$ & $2.5 / 17.9$ & $3.1 \pm 0.4$ \\
\hline $19 / 20$ & turf mat & 13 & $6.4 \pm 4$ & $1.9 / 14.4$ & $3.1 \pm 0.4$ \\
\hline $19 / 20$ & $\begin{array}{c}\text { bottle }+ \text { turf } \\
\text { mat }\end{array}$ & 13 & $7.4 \pm 4.6$ & $1.9 / 17.9$ & $3.1 \pm 0.4$ \\
\hline $16 / 20(4 a)^{* * *}$ & $\begin{array}{c}\text { bottle }+ \text { turf } \\
\text { mat }\end{array}$ & 27 & $2.8 \pm 2$ & $0.1 / 17.9$ & $2.8 \pm 0.3$ \\
\hline
\end{tabular}

*The number of sampled storm surges in 19/20_T refers to the seven measured events given in Table $5-1$.

**Winter season 19/20 includes all events that occurred in 2019/20.

$* * * 16 / 20$ (4a) shows the calculated average vertical accretion rate of all measuring seasons from 2016 to 2020.

$* * * *$ The average inundation height in $\mathrm{m}$ above mean sea level is based on data from both interior tide gauges Kirchhofswarf and Kirchwarf. 


\subsection{Discussion}

\subsection{1 $\mathrm{SPM}_{\mathrm{i}}$}

Previous studies have shown that meteorological forcing strongly affects SPM concentrations (e.g. Jorge and van Beusekom 1995; Fettweis et al. 2006, 2007, 2012). A strong correlation between an increasing wind speed especially from southwestern directions and increasing SPM $_{i}$ in the tidal flat around Langeness has been demonstrated by Hache et al. $(2019,2020)$. Meteorological forcing is generally higher during storm surges compared to moderate inundations, which most likely explains the strong increase in $\mathrm{SPM}_{i}$ up to a factor of 3.5. SPM measurements at a storm surge on December 3-4, 1999 on a tidal flat in the inner part of Hobo Dyb on the Danish west coast also showed high SPM concentrations during the flood phase and significant lower SPM values during the ebb phase (Bartholdy and Aagaard 2001). These authors determined the maximum SPM concentration at the combined maximum wave- and current-induced bed shear stress. The highest $\mathrm{SPM}_{\mathrm{i}}$ of our measurements was recorded during the flood phase of the storm surge SF1 (Figure 5-4) along with the highest wind speeds, wind direction from southwest, and the largest tidal range of all measured events (Table 5-1). This underlines the strong impact of meteorological- and hydrodynamic forcing on $\mathrm{SPM}_{\mathrm{i}}$. The individual proportions of $\mathrm{SPM}_{\mathrm{i}}$, derived from advection processes or local erosion in front of the Hallig margins, cannot be assessed with our stationary measurements. The impact of meteorological- and hydrodynamic forcing on the tidal flats in front of Langeness is illustrated before (Figure 5-8a) and after the storm surge SF3 (Figure 5-8b, c). The hydrodynamic forces were strong enough to erode the surface sediments and expose the peat below and even create deeper erosion patterns near the tidal channel. These observations underline the assumption that a considerable proportion of $\mathrm{SPM}_{\mathrm{i}}$ is most likely generated locally on the tidal flats in front of the Hallig margin. 

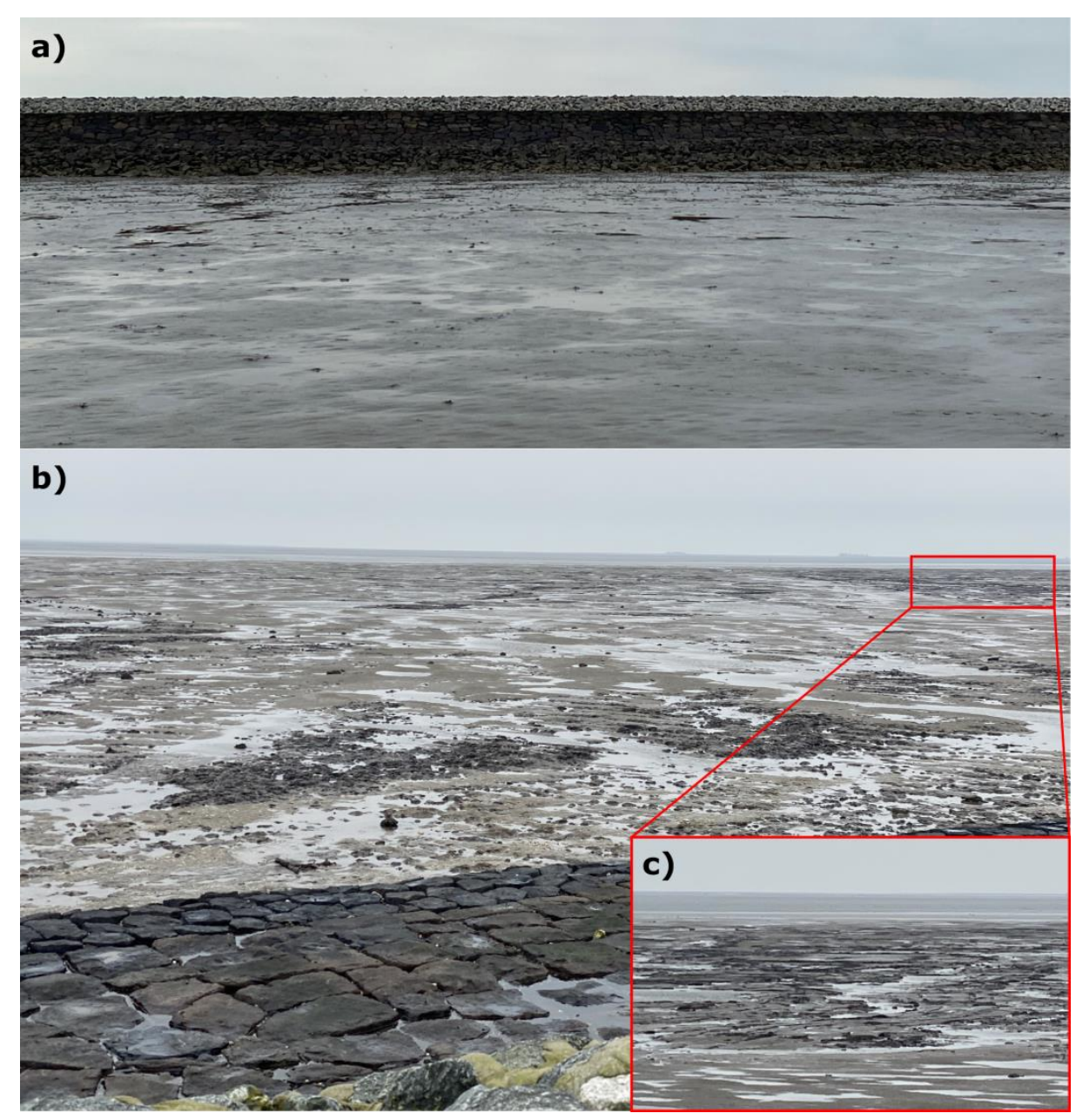

Figure 5-8: Impressions of the tidal flat to the southeast of Langeness (for location see magenta box in Figure 5-2) during low water before the storm surge SF3 with the revetment and breakwater construction in the background (a). The tidal flat was predominantly covered with fine sand and some silt-sized material on top. (b) shows the same area during low water after the storm surge SF3 but viewed from the top of the breakwater construction towards the tidal flat. The silt-sized material is removed and darker parts in the tidal flat indicate spots where erosion has exposed the peat below. The inset (c) shows an area close to the tidal channel with deep erosion patterns in the tidal flat.

\subsection{2 $\mathrm{SPM}_{\mathrm{rs}}$}

In general, $\mathrm{SPM}_{\mathrm{rs}}$ increases with increasing $\mathrm{SPM}_{\mathrm{i}}$ (Figure 5-4). This result is consistent with Temmerman et al. (2003a), who demonstrated that SPM in the water that floods the marsh surface at the beginning of an inundation increases linearly with maximum inundation height. The linear increase in SPM is in turn consistent with recent particle tracking model results by (Hache et al. 2021). In that study, the accumulated particle quantities of seven inundations correlate positively with increasing inundation heights with a coefficient of determination after Pearson $\left(R^{2}\right)$ of 0.87 . 
In the west of Langeness, SPM increases on average over all analyzed storm surges from about $9 \%$ of $\mathrm{SPM}_{\mathrm{i}}$ at moderate inundations to $28 \%$ at storm surges. In the southeast of Langeness, $\mathrm{SPM}_{\mathrm{rs}}$ increases on average from $20 \%$ to $49 \%$ with a maximum of $73 \%$ at storm surge SF1

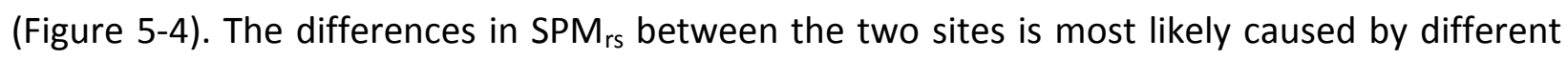
distances to the Hallig margin, which are $50 \mathrm{~m}$ at station T-8 (southeast) and $150 \mathrm{~m}$ at station T7 (west). This interpretation is consistent with other studies. For instance, (i) Christiansen et al. (2000) measured decreasing sediment concentrations along a transect with an increasing distance to a tidal creek located in the Phillips Creek area of Hog Island Bay on the Atlantic side of Delmarva Peninsula, Virginia, US; (ii) Temmerman et al. (2003b) measured a strong decrease in sedimentation rates with increasing distance from the nearest creek or marsh edge at Scheldt estuary located in the southwest of the Netherlands and the northwest of Belgium; (iii) Butzeck et al. (2015) measured decreasing sediment deposition rates with increasing distance from the sediment source at three marsh types (tidal freshwater marsh, brackish marsh and salt marsh) located at Elbe Estuary, Germany; and (iv) Schindler et al. (2014a) who also measured decreasing vertical accretion rates with increasing distance to the Hallig margin at Langeness and the adjacent Hallig Hooge (Figure 5-1).

Comparisons of the time needed for flooding indicate that shorter time and thus higher flow speed from the tidal flat towards the marsh surface leads to an increase in SPM rs. The averaged $\mathrm{SPM}_{\mathrm{rs}}$ at stations T-7 and T8 increase from SF2 $(0.3 \mathrm{~g} / \mathrm{l})$ with a flooding duration of $70 \pm 3$ minutes to SF3a $(0.35 \mathrm{~g} / \mathrm{l})$ with $65 \pm 8$ minutes (Table $5-3)$. Finally, a maximum in SPM $\mathrm{S}_{\mathrm{rs}}$ at SF1 $(0.55 \mathrm{~g} / \mathrm{l})$ is reached along with the shortest duration of $52 \pm 2$ minutes until the Hallig is completely inundated.

\subsubsection{SPM $_{\mathrm{fl}}$}

Tidal gates control the water levels on the marshland during rising and falling tides and thus affect the flow velocity of the water draining from the Hallig after inundations. The high flow velocity in the drainage channel during the ebb phase induced by the widely opened tidal gates may cause erosion and/or resuspension in the drainage channels and may also affect the adjacent marshland close to the channels. The SPM concentration in the drainage channel landside of a tidal gate revealed constantly higher values than at the adjacent station on the marshland (see black box in Figure 5-5). Consequently, suspended particles in the drainage water flow are flushed via the drainage channels and the tidal gates towards the tidal flats. 
The comparison of $\mathrm{SPM}_{\mathrm{rs}}$ vs. SPM $\mathrm{SP}_{\mathrm{fl}}$ clearly demonstrates higher values during the flood phase than during the ebb phase, independent of the calibration type (Figure 5-6). The coarsecalibrated $\mathrm{SPM}_{\mathrm{fl}}$, however, shows only slightly lower values in comparison to $\mathrm{SPM}_{\mathrm{rs}}$ (squares close to 1:1 line in Figure 5-6). Considering that we have determined high sediment accumulation rates after storm surge events calls for far lower $\mathrm{SPM}_{\mathrm{fl}}$ compared to $\mathrm{SPM}_{\mathrm{rs}}$. Therefore, we have to assess $\mathrm{SPM}_{\mathrm{fl}}$ differently. It is well known that OBS devices tend to underestimate coarser particles depending on the material used for calibration (e.g. Bunt et al. 1999; Fugate and Friedrichs 2002; Voulgaris and Meyers 2004; Downing 2006; Baeye et al. 2011). Because of the strong hydrodynamic conditions at storm surges, $\mathrm{SPM}_{\mathrm{i}}$ and $\mathrm{SPM}_{\mathrm{rs}}$ contains coarser GSD compared to moderate inundations (Figure 5-7). During high water, hydrodynamic conditions calm down due to decreased tidal currents. Furthermore, the asymmetric interior gauge level curves (especially for storm surges; Figure 5-3) proofs a prolonged ebb phase compared to the flood phase. This results in lower current velocities on most parts of the marshland except for drainage channels and adjacent marshland affected by the open tidal gates. These lower energetic conditions allow coarse particles to settle. Thus, the GSD of SPM should be finer within the water column of the drainage water masses after high water. The OBS sensors, however, are calibrated by default with sediment accumulator SB samples including material that is collected during the whole storm surge (flood and ebb phase), i.e. with material being coarser than that representative for the drainage water SPM. Hence, we can assume that the finer GSD in the drainage water masses lead to an overestimation of the determined $\mathrm{SPM}_{\mathrm{fl}}$ (squares close to 1:1 line in Figure 5-6). Consequently, for $\mathrm{SPM}_{\mathrm{fl}}$ we applied a calibration with finer sediment collected in the sediment accumulators during moderate inundations at each of the marshland stations (Table 5-2). By applying this approach, we generated fine-calibrated SPM values for all storm surges to define a range of possible $\mathrm{SPM}_{\mathrm{fl}}$ on the marshland (triangles in Figure 5-6).

The ratios between the coarse- and fine-calibrated $\mathrm{SPM}_{\mathrm{fl}}$ are 2.6 for station T-7 and 4.2 for station T-8. The higher ratio at T-8 is most likely caused by stronger variations in hydrodynamic forcing due to its closer position to the Hallig margin. Stronger variations in hydrodynamic forcing lead to higher variations in the inherent particle properties like size, shape, density and composition, which strongly affect the OBS response (e.g. Druine et al. 2018). These variations may result in over- or underestimation of SPM concentrations by a factor of 2 or more (Fettweis et al. 2019). With an increasing distance to the Hallig margin, hydrodynamic 
variations as well as variations in inherent particle properties are less pronounced which is considered the most likely cause for the lower ratio between the coarse- and fine-calibrated SPM concentrations at station T-7.

The suspension samples taken during the storm surge SF3 landside of the tidal gate show dry mass concentrations between 0.028 and $0.053 \mathrm{~g} / \mathrm{l}$. These values roughly resemble the finecalibration in Figure 5-6 and indicate that the coarse-calibration is not appropriate to estimate $\mathrm{SPM}_{\mathrm{fl}}$. In fact, they are slightly below the fine-calibrated $\mathrm{SPM}_{\mathrm{fl}}$, which might be caused by the fact that the suspension samples were taken 5 to 6 hours after high water at some safety distance from the rapidly draining water masses. Considering that the suspension samples reflect single-point measurements and the $\mathrm{SPM}_{\mathrm{fl}}$ values reflect averaged concentrations over hundreds of SPM measurements during the ebb phase, the concordance of both values is quite stunning.

\subsubsection{Sediment accumulation}

The average sediment accumulation of all trap clusters increases at storm surge conditions by a factor of 8.9 compared to moderate inundations (Table 5-3). An increasing sediment accumulation with an increasing tidal range was also observed for surface sediment depositions in the Bay of Fundy by Chmura et al. (2001). An increase of sediment accumulation with increasing water level is also in line with the simulation results by Temmerman et al. (2003a). The high increase in sediment accumulation is also in line with the particle tracking results from a detailed hydrodynamic numerical model of Langeness and the surrounding tidal flats (Hache et al. 2021). The model output suggests a seven times higher particle accumulation for storm surge conditions in comparison to moderate inundations. The positive correlation between sediment accumulation and $\mathrm{SPM}_{\mathrm{rs}}$ at both marshland sites show that, at given values of $\mathrm{SPM} \mathrm{r}_{\mathrm{rs}}$ sediment accumulation is significantly higher at station T-8 compared to T-7 (Figure 5-9). This is again most likely due to its proximity to the Hallig margin. The correlation between sediment accumulation and SPM $\mathrm{r}_{\mathrm{s}}$ is better constrained for station T-8 because at T-7 the five consecutive storm surge tides SF3a to 3e were all calibrated with the same calibration sample (Table 5-2). 


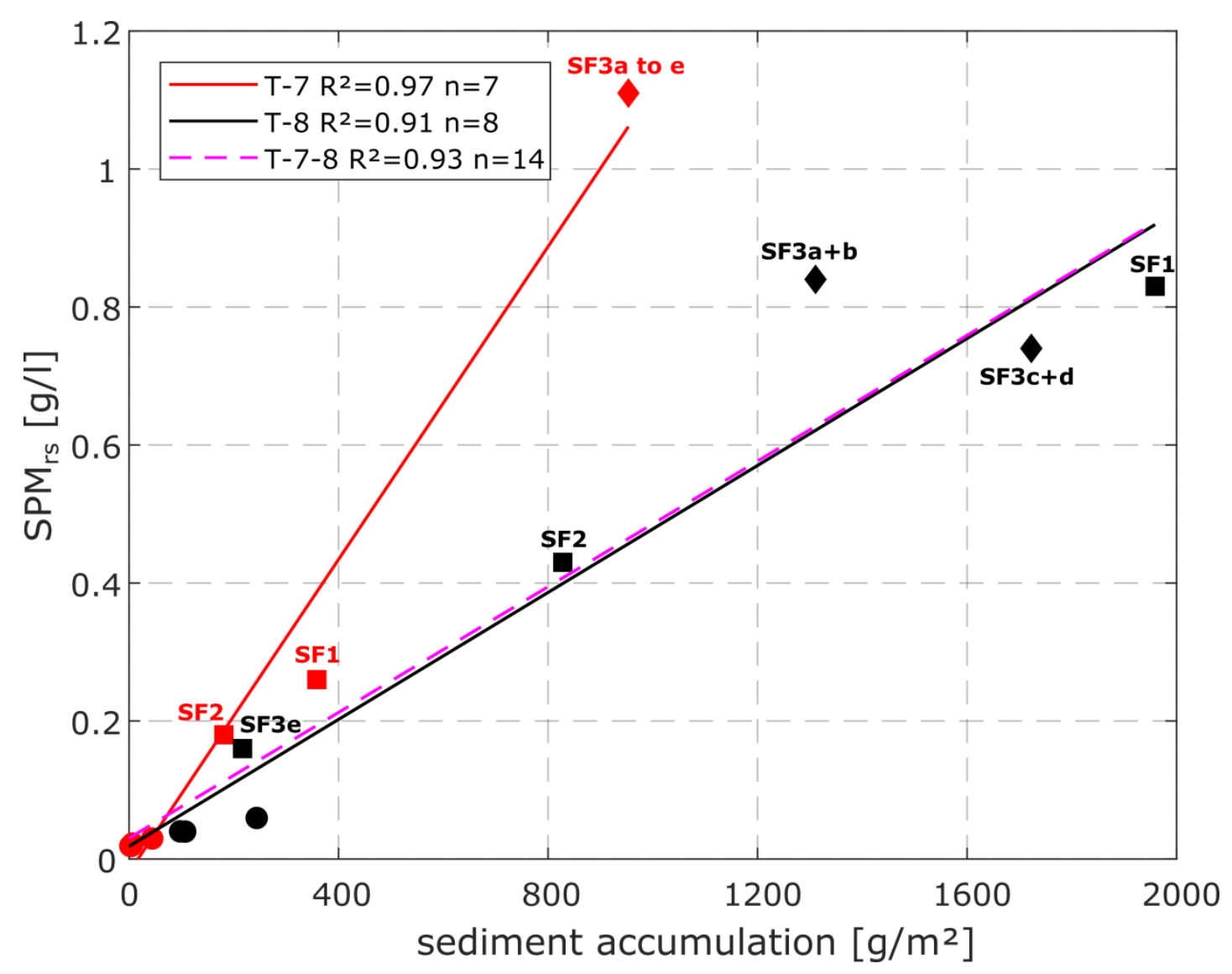

Figure 5-9: Linear regression analysis between $\mathrm{SPM}_{\mathrm{rs}}$ at the two stations on the marshland (T-7 and T-8, red and black lines, respectively) and sediment accumulation of bottle traps next to both stations for all storm surge events and the moderate inundations during the winter season 2018/19 (Hache et al. 2020). Storm surges that could be sampled after one tide are shown with squares. Sampled storm surges that contain sediment accumulation data and $\mathrm{SPM}_{\mathrm{rs}}$ of at least two or more tides are shown by diamonds. The moderate inundations are shown by circles. Dashed magenta line refers to T-7-8 (all data from T-7 and T-8 except for the summed storm surge SF3a to SF3e from T-7). $\mathrm{R}^{2}$ is the adjusted coefficient of determination after Pearson and $\mathrm{n}$ is the number of observations.

At moderate inundations, the average sediment accumulation at station T-7 is only $8 \%$ of the value at station T-8 and increases to $25 \%$ during storm surges (Table 5-3). This suggests an improved SPM transport to the inner marsh under storm surge conditions. This is supported by comparing the GSD of sediment accumulator samples taken on the tidal flats and the sediment trap samples taken on the marshland (Figure 5-7). At moderate inundation (Figure 5-7a) the two sediment accumulators SB and ST show distinct differences suggesting that the water column was not homogeneously mixed. Nevertheless, fine sand reached the upper water column at the ST accumulator. On the marshland, the median grain size strongly decreases to coarse silt. The decrease in grain size is most likely caused by the breakwater constructions at the Hallig margin, which hamper SPM in reaching the marshland with respect to both mass and 
grain size (Hache et al. 2020). At higher water levels (SF2), the difference in GSD between ST and SB is less pronounced (Figure 5-7b) indicating that the water column is better mixed. The adjacent sediment trap samples show almost the same median grain size than ST with still slightly smaller grain size behind the higher breakwater $(1 \mathrm{H})$ compared to the lower breakwater (1L). At storm surge SF1 with the highest water level, the GSD of both sediment accumulators is (i) the coarsest of all measured storm surges and (ii) shows the least contrast between SB and ST implying almost perfect mixing within the water column (Figure 5-7c). Unlike all other measured events, the sediment trap samples behind differing breakwater heights show uniform GSD with highest median values. Obviously, the hampering effect of the breakwater construction is strongly reduced at the high water levels and strong hydrodynamic forcing of SF1.

\subsubsection{Vertical accretion}

Seven described storm surge induced inundations generated vertical accretion rates of $5.9 \pm 3.8$ $\mathrm{mm}$ in the winter season 2019/20, what roughly matches the local MHW rise of $5 \pm 0.3 \mathrm{~mm} / \mathrm{a}$ (Schindler et al. 2014a). Taking into account the six additional inundations in the second half of the same winter season, a maximum vertical accretion rate of $8.4 \pm 5.1 \mathrm{~mm}$ was measured based on bottle traps and $7.4 \pm 4.6 \mathrm{~mm} /$ a on turf mats (Table 5-4). According to Schindler et al. (2014b), the sediment retention capacity of the turf mats decreases when the sediment accumulation exceeds $2.0 \mathrm{~kg} / \mathrm{m}^{2}$ due to remobilization effects at higher storm surge frequency. The high storm surge frequency in the winter season 2019/20 resulted in average accumulation rates of $4.0 \pm 2.6 \mathrm{~kg} / \mathrm{m}^{2}$ calculated from all turf mats. An average vertical accretion rate based on all trap data of $7.4 \pm 4.6 \mathrm{~mm} / \mathrm{a}$ is calculated for the winter season $2019 / 20$. Considering the limited retention capacity of the turf mats this is a rather conservative estimation. Anyway, such high accretion rates have not been reported in previous years (Schindler et al. 2014a; Hache et al. 2020), and seem to be rather exceptional, for instance in years with increasing storm intensity and frequency (e.g. Schuerch et al. 2013). The average vertical accretion rate for the last four years of $2.8 \pm 2 \mathrm{~mm} / \mathrm{a}$ (Table 5-4) is relatively high and strongly influenced by the exceptional high storm surge frequency in the winter season 2019/20. Schuerch et al. (2012) show that temporal variations of up to five-fold higher accretion rates in cores from Sylt, Germany match periods of high storm activity and therefore only during these periods have the potential to compensate for MSL rise. Including annual accretion rates of Langeness from 
Schindler et al. (2014a) with $0.7 \mathrm{~mm} / \mathrm{a}$ in 2011, $1.8 \mathrm{~mm} / \mathrm{a}$ in 2012 and $1.1 \mathrm{~mm} / \mathrm{a}$ in 2013 to our data set results in a seven year averaged vertical accretion rate of $2.1 \pm 1.5 \mathrm{~mm} / \mathrm{a}$. This seven year averaged accretion rate, however, is considerably below the MHW rise and underlines the need to mitigate the imbalance between sediment accretion and sea level rise.

\subsubsection{Possible mitigation strategies}

Counteracting SLR with a sole focus on conventional coastal engineering solutions and their expensive maintenance may become unsustainable on the long-term (Temmerman et al. 2013; Smith et al. 2017). Hence, a sole focus on higher dyke constructions might not be a viable option for the Halligen (Hofstede et al. 2019b; Hofstede 2019) especially with regard to their characteristic of limiting sediment accumulation (Hache et al. 2020). Given the likelihood that storm frequencies and storm surge water levels will increase in the future (Hunter 2009; Arns et al. 2015a), the ability of coastal marshes to better adapt to SLR by sediment accretion might increase, too (e.g. Schuerch et al. 2013). To further increase the natural adaptability of the Halligen, strategies have to be developed to substantially increase the sediment transport onto the Halligen and to reduce SPM losses in the drainage water flow after inundations.

An increase of SPMrs might be ensured by an adjustment of breakwater constructions. Results of a numerical model using particle tracking point to a possible increase of SPM $\mathrm{r}_{\mathrm{rs}}$ of up to almost $100 \%$ at moderate inundations if the breakwater constructions would be lowered (Hache et al. (2021). According to that study, the increase of SPMrs was mainly caused by a significant increase of tracked $30 \mu \mathrm{m}$ particles towards the marsh surface. Storm surge simulations in the same study showed a sediment accumulation rate of almost $700 \%$ compared to moderate inundations. This coincides with the observed sediment accumulation in this study (Table 5-3). An additional increase of tracked fine sands up to $50 \%$ was achieved in storm surge simulations investigated by using particle tracking with $80 \mu \mathrm{m}$ particles (Hache et al. 2021). Currently, the majority of available fine sand cannot overcome the breakwater constructions (Hache et al. 2020). Considerable amounts of fine sand only reach the marshland during rarely occurring storm surges such as SF1 (Figure 5-7).

The increased SPM $\mathrm{rs}$ at higher water levels also coincides with a faster marshland flooding. Generating additional inlets through open tidal gates during the flood phase of an inundation could be an option to allow an even faster flooding of the marshland. This in turn increases 
$\mathrm{SPM}_{\mathrm{rs}}$ and may result in a more uniform sediment distribution on the Hallig surface by utilizing the drainage channels on the Hallig as SPM "transport paths" (Stoddart et al. 1989). This assumption is supported by findings of Temmerman et al. (2005a) who showed that a high marsh platform is mainly supplied with water and sediment over its creek system and the supply via the marsh edge primarily increases with an increasing high water level. Furthermore, the sediment accumulation rates may be increased by slowing down the drainage water masses and thus decreasing $\mathrm{SPM}_{\mathrm{fl}}$. These results support previous suggestions for changes in managing the tidal gates to increase sediment accumulation on the marshland (Schindler 2014; Hofstede et al. 2019b).

Five moderate inundations occurred in the measuring season $2018 / 19$, which resulted in an annually average vertical accretion of $1 \mathrm{~mm}$ (Table 5-4). This annually average accretion rate resulted in the lowest average accretion rate per inundation of $0.2 \mathrm{~mm}$ within the period from October 2016 to March 2020. Based on the fine-calibration attempt to assess SPM $_{\mathrm{fl}}$ during storm surges (Figure 5-6), we demonstrate that $\mathrm{SPM}_{\mathrm{fl}}$ is comparable with $\mathrm{SPM}_{\mathrm{rs}}$ at moderate inundations. Considering that Langeness was inundated 10 times under storm surge conditions in 2019/20, an additional increase of ten times of the lowest average accretion per inundation (i.e. $2 \mathrm{~mm}$ or $27 \%$ of the total vertical accretion in 2019/20) might be possible if $\mathrm{SPM}_{\mathrm{fl}}$ could be decreased to zero. Note that this theoretical approach forms a conservative estimation based on the lowest average accretion per inundation of $0.2 \mathrm{~mm}$. In addition, the sediment accumulation on turf mats, which are more prone to erosion (Nolte et al. 2013a; Schindler et al. 2014b), resulted in a $2 \mathrm{~mm}$ lower annual accretion rate compared to the bottle traps (Table 5-4). This underlines the assumption that additional vertical accretion could be generated by reducing $S P M_{\mathrm{fl}} . \mathrm{SPM}_{\mathrm{fl}}$ caused by redistribution might be reduced with an adapted marshland vegetation on the Halligen as vegetation characteristics such as vegetation height and density are able to attenuate hydrodynamic forcing e.g. flow velocity (Temmerman et al. 2005b) or wave height (e.g. Marsooli and Wu 2014; Anderson and Smith 2014), even at storm surges (Möller et al. 2014). We want to point out that based on this work, an assessment of altered erosion processes due to the discussed mitigation strategies cannot be given. The complex interrelationships between the discussed strategies and a possible increased erosion potential must therefore carefully investigated in future research projects. 


\subsection{Conclusions}

The investigation of seven storm surge induced inundations on Hallig Langeness with peak water levels of $>1.5 \mathrm{~m}$ above MHW uncovers large SPM concentrations $\left(\mathrm{SPM}_{\mathrm{i}}\right)$ on the tidal flat. These have the potential to generate vertical accretion rates on the Hallig marshland that could keep pace with the local MHW rise. The main findings that distinguish the measured storm surges from moderate inundations (water levels $<1.5 \mathrm{~m}$ above MHW) are (i) up to 3.5 fold increased $\mathrm{SPM}_{i}$ on the tidal flats, (ii) increase of SPM concentrations on the marshland (SPM $\mathrm{rs}_{\mathrm{rs}}$ to $28 \%-49 \%$ of $\mathrm{SPM}_{\mathrm{i}}$, (9\%-20\% for moderate inundations) on average over all storm surges, depending on the distance to the Hallig margin, (iii) a maximum of SPM $\mathrm{rs}_{\mathrm{r}}$ of $73 \%$ of $S P M_{i}$ during the strongest storm surge SF1 with respect to highest wind speed, largest tidal range and fastest flooding time, (iv) compared to moderate inundations the fine sand fraction reaches the marshland during storm surges probably because the hampering effect of breakwater constructions decreases with higher water levels and stronger hydrodynamic forcing, $(v)$ the average vertical accretion rate per inundation increases from approximately $0.2 \mathrm{~mm}$ for moderate inundations to $0.84 \mathrm{~mm}$ for storm surges, and (vi) $\mathrm{SPM}_{\mathrm{fl}}$ within the drainage water masses during the ebb phase of storm surges is of the same order as SPMrs at moderate inundations.

Theoretically, a complete prevention of $\mathrm{SPM}_{\mathrm{fl}}$ would have increased the average annual vertical accretion in the measuring season $2019 / 20$ by approximately $2 \mathrm{~mm}$ ( $27 \%$ of the effective vertical accretion in 2019/20).

In all cases - moderate as well as storm surges - breakwater constructions seem to hinder the exploitation of the full potential of SPM based sediment accretion. Therefore, we argue that a sole focus on the protective properties of coastal protection structures endangers the longterm preservation of anthropogenically modified marshes like the Halligen. This conclusion supports the current rethinking of coastal protection strategies in the responsible public authorities. In general, new measures should aim to balance the different needs of erosion protection, land usage and vertical accretion in lowlands without sea dykes in a way that allows to preserve their unique character. In case of the Halligen lowering the breakwater constructions could provide one option. An adapted management of tidal gates would be another option aiming at (i) faster flooding of the marsh surface during inundations and (ii) 
slowing down the drainage water flow after inundations. Both measures are able to substantially increase the vertical accretion of the marshland surface.

\section{Acknowledgements}

The study forms part of the joint research project "Real life coastal laboratory to develop shortand long term strategies for the protection and preservation of Schleswig-Holsteins Halligen (Living Coast Lab Hallig)" funded by the German Federal Ministry of Education and Research (BMBF, 03F0759C). We wish to thank the inhabitants of Langeness for their hospitality and helpfulness in supporting the research project. We thank the responsible authority for coastal protection, national parks and marine protection in Schleswig Holstein (LKN.SH) for providing sea state and gauge level data. We thank the German Meteorological Service (DWD) for providing wind data. Finally, we acknowledge thoughtful and in-depth comments and suggestions by an anonymous reviewer on an earlier version of the manuscript. 


\section{$6 \quad$ Plant traits affect surface elevation change in salt marshes: an example of biogeomorphic ecosystems}

This paper is in preparation

Julia Bass ${ }^{1}$, Dirk Granse ${ }^{2}$, Ingo Hache ${ }^{3}$, Kai Jensen ${ }^{2}$, Volker Karius ${ }^{3}$, Vanessa Minden ${ }^{1,4}$, Martin Stock $^{5}$, Sigrid Suchrow ${ }^{2}$, Michael Kleyer ${ }^{1}$

Corresponding author: Julia Bass, University of Oldenburg, Landscape Ecology Group, Carl-vonOssietzky-Straße 9-11, 26111 Oldenburg, Germany, julia-1.bass@uni-ulm.de

${ }^{1}$ Landscape Ecology Group, University of Oldenburg, Carl-von-Ossietzky-Straße 9-11, 26111 Oldenburg, Germany

${ }^{2}$ Applied Plant Ecology, Institute of Plant Science and Microbiology, University of Hamburg, Ohnhorststraße. 18, 22609 Hamburg, Germany

${ }^{3}$ Geoscience Center University of Göttingen, Department of Sedimentology and Environmental Geology, Goldschmidtstraße 3, 37077 Göttingen, Germany

${ }^{4}$ Department of Biology, Ecology and Biodiversity, Vrije Universiteit Brussel, Pleinlaan 2, 1050 Brussels, Belgium

${ }^{5}$ Schleswig-Holstein Agency for Coastal Defence, National Park and Marine Conservation, National Park Authority, Schlossgarten 1, 25832 Tönning, Germany

Keywords: Biogeomorphology; Plant functional traits; Accretion; Erosion; Compaction; Coastal squeeze; Germany, North Sea, Wadden Sea: $53^{\circ}-55^{\circ} \mathrm{N}, 7^{\circ}-9^{\circ} \mathrm{E}$ 


\section{Abstract}

The current climate crisis is associated with rising sea levels, which raises the concerning prospect of losing coastal ecosystems, such as salt marshes. Salt marshes fulfill multiple ecosystem services including providing habitat for specialized plants and animals, attenuating wave energy, and protecting coastal areas from flooding. Where inland migration is impossible, salt marshes will only persist if their surface elevation gain exceeds the sea-level rise. Surface elevation change (SEC) is the result of positive SEC, driven by sedimentation, and negative SEC, driven by erosion and compaction. These processes can be influenced by abiotic and biotic factors. The biotic factors, best described by plant functional traits of the salt-marsh vegetation, are, however, not well understood. We assembled a large data set of SEC, plant abundance, and trait measurements from the German Wadden Sea, covering three salt-marsh types, i.e. island salt marshes, mainland salt marshes, and Hallig salt marshes, representing natural unmanaged, anthropogenic, and grazed marshes. By using multiple regression analyses, we studied the effects on SEC of functional traits and distance to the marsh edge. The mean SEC in all three salt-marsh types was below the observed sea level rise (SLR). This questions the ability of salt marshes to accrete as rapidly as recent SLR. There were, however, pronounced local differences in SEC, which were jointly explained by the distance to the marsh edge and by plant traits describing above-ground roughness and below-ground anchoring capacity of the vegetation. Higher roughness supported positive SEC; stronger anchoring capacity can reduce negative SEC. To better predict coastal resilience to SLR, these findings suggest including plant functional traits in models of salt marsh SEC.

\subsection{Introduction}

Recent loss of salt marshes (Watson et al. 2017) has induced widespread concern about their ability to withstand sea-level rise (SLR) (Kirwan et al. 2016a; Murray et al. 2014; Suchrow et al. 2012). By the end of this century, the global mean sea level will most likely rise between 0.29 $\mathrm{m}$ and $1.1 \mathrm{~m}$ (IPCC, in press), which in the same time period would result in a 20 to $50 \%$ loss of marsh area worldwide (Craft et al. 2009; McFadden et al. 2007). These predictions are of great concern, because marshes have important ecosystem functions, such as buffering coasts from 
storm surges, attenuating wave energy, sequestering carbon, improving water quality, and providing habitat for specialized plants and animals (Barbier et al. 2011; Elschot et al. 2013).

Historically, tidal marshes have responded to increases in sea level by inland migration and/or accreting sediment (Donnelly and Bertness 2001). Likewise, salt marshes will survive future SLR only if vertical accretion surpasses SLR, or if their inland migration is faster than their seaward loss. Due to infrastructure such as dikes, roads or settlements, however, this landwards retreat is often not possible, leading potentially to the loss of suitable habitat for salt-marsh species and, ultimately, to the loss of the salt marshes (Borchert et al. 2018). Thus, in many areas, only vertical accretion ensures the long-term stability and survival of salt marshes (Morris et al. 2002).

Positive or negative surface elevation change (SEC) of salt marshes depends mainly on sedimentation, or erosion and soil compaction, respectively. Abiotic factors, such as the concentration of suspended sediment and sediment deposition were found to be positively correlated with positive SEC (Andersen et al. 2011). The distance from the intertidal mudflats (Kirwan et al. 2016a) or from main creeks, the elevation, as well as tidal currents and waves were found to be negatively correlated with negative SEC (Cadol et al. 2014; Esselink et al. 1998; Kolker et al. 2010). Biotic factors are considered with regard to their influence on salt marsh SEC, since salt marshes are a classic example of biogeomorphic ecosystems. In biogeomorphic ecosystems, interactions between plants and geomorphic processes are known to govern the functioning of the system (Corenblit et al. 2015). Marsh vegetation is believed to influence SEC in three ways. First, it is assumed to directly enhance positive SEC, as it contributes to soil build-up through the production of organic matter (Langley et al. 2009; Nyman et al. 2006; Schile et al. 2014). Second, vegetation roughness and plant anchoring in the soil also indirectly affects positive SEC by attenuating wave energy and slowing water flow. This leads to a decline in bed shear stress and the enhancement of mineral sediment trapping (Mudd et al. 2010). Lastly, the reduction of bed shear stress and the anchoring effect of the roots also prevent the resuspension of deposited sediments, and the direct erosion of, salt marsh surfaces (Francalanci et al. 2013; Temmerman et al. 2012).

Including vegetation roughness in models of open-channel water flow has a long tradition (Manning 1891; Strickler 1923). Surface roughness, including vegetation, is expressed as an empirically derived coefficient in the Gauckler-Manning-Strickler formula, which is still widely 
applied in hydraulics (Bleninger et al. 2006). One drawback of the formula is its use of rather broad vegetation categories (e.g., 'short grass', 'high grass' for pastures in floodplains), leaving ample room for subjective interpretation (Chow 1959). Plant functional traits (Lavorel et al. 1997) may allow a more mechanistic understanding of roughness or anchoring, because they are measurable properties of the plants that determine the vegetation of a site (Garnier et al. 2007; Kleyer 1999). However, only a few studies have used a trait-based approach to assess biologically mediated SEC and wave attenuation in salt marshes (Bouma et al. 2005; Mudd et al. 2010). In salt marshes, wave attenuation was found to be positively correlated with leaf- and stem stiffness (Paul et al. 2012; Rupprecht et al. 2017), vegetation height (Rupprecht et al. 2017), as well as shoot density and shoot biomass (Paul et al. 2012). Increased near-bed turbulences, accompanied by a reduced velocity within the canopy, were found to decrease sedimentation within vegetated areas (Lefebvre et al. 2010). Conversely, Reef et al. (2018) found no significant effect of vegetation height and biomass on salt marsh SEC, although they did detect deposition of material on the plant/soil surfaces during flood tides. Even negative effects of plants on salt marsh SEC rates were found in the event that plant movements scour the surface when acted on by waves and currents (Temmerman et al. 2007). This holds true for patchy vegetation, as found in pioneer zones and along marsh seaward margins. Widdows et al. (2008) pointed out that linkages between plant traits and small-scale effects around plants do not necessarily translate into vegetation effects on hydrodynamic conditions measured on a larger scale.

These contrasting results demonstrate a lack of understanding of the interactions between vegetation and salt marsh SEC. The current fragmented knowledge, therefore, prevents many modelers from incorporating biogeomorphic feedback in their models of salt marsh resilience to SLR.

Salt marshes occur in different locations along the German North Sea coast. This study looked at three different salt marsh types. First, salt marshes on an uninhabited barrier island that have remained uninfluenced by direct human activities. Such island salt marshes are protected from the surf by dunes on the windward side of the island, but diurnal tides and storm surges do contribute to SEC. Here, SEC of +1 to $+16 \mathrm{~mm} / \mathrm{a}$ was found (Schuerch et al. 2012). Second, we studied salt marshes along the mainland coastline that have experienced land reclamation and embankment activities. Here, an average SEC of $+6 \mathrm{~mm} / \mathrm{a}$ had been reported (Suchrow et al. 2012). Last, we examined salt marshes on Halligen, small, island-like marsh areas that 
became disconnected from the German mainland marsh during past storm-surge events. These marshes are often grazed and become submerged only during storm surges. Here, average SECs of $+1 \mathrm{~mm} / \mathrm{a}$ (Hallig Hooge), $+1 \mathrm{~mm} / \mathrm{a}$ (Hallig Langeness), and $+3 \mathrm{~mm} / \mathrm{a}$ (Hallig Nordstrandischmoor) were found (Schindler et al. 2014b). Given the differences between the three salt marsh types in flooding regime and in yearly SEC, it is appropriate to study them separately.

To foster a better understanding of vegetation-SEC interactions, this study aimed to elucidate the effects of plant traits on SEC of salt marshes on islands, mainland coasts, and Halligen. Specifically, we tested the following hypotheses: (1) SEC decreases with distance from the shore. (2) Positive SEC in salt marshes is facilitated by rough vegetation, which is composed of plants with a high canopy height, large stem biomass, stiff stems, and large leaf areas. (3) Negative SEC is reduced through anchoring effects of the below-ground parts of the vegetation. In particular, plants with high below-ground biomass allocation stabilize the marsh soil and reduce negative SEC.

\subsection{Methods}

\subsubsection{Study area}

To elucidate the effect of plant functional traits on SEC of island, mainland, and Halligen salt marshes, we analyzed a total of 335 plots located along the German North Sea coast (see Table 6-1 and Figure 6-1). On the island of Mellum, 38 plots on a gradient from the pioneer zone to the upper salt marsh were delimited. On the mainland, 184 plots were located in salt marshes of Lower Saxony at Leybucht, Norderland, and the Jade Bight, as well as along the coastline of Schleswig-Holstein. These plots mainly covered both lower and upper salt marsh communities. The Halligen Langeness, Nordstrandischmoor, and Hooge provided 113 plots covering mostly upper salt marsh communities. Vegetation or SEC data originated either from published sources or were unpublished (see references in Table 6-1). In addition, all community-weighted trait data were unpublished. 
Table 6-1: Plot locations, number of replicates, and data sources for the three salt marsh types; island, mainland (LS: Lower Saxony), and Halligen. In addition to the sources for vegetation and SEC data, all community-weighted trait data were unpublished. Ntotal = total number of plots of the study area, NPSEC = number of plots which experienced positive SEC, NNSEC $=$ number of plots which experienced negative SEC.

\begin{tabular}{|c|c|c|c|c|c|c|c|}
\hline $\begin{array}{l}\text { Salt } \\
\text { marsh } \\
\text { type }\end{array}$ & Study area & $\begin{array}{l}\text { Study area } \\
\text { coordinates }\end{array}$ & $\mathbf{N}_{\text {total }}$ & $\mathbf{N}_{\text {PSEC }}$ & $\mathbf{N}_{\text {NSEC }}$ & $\begin{array}{l}\text { Vegetation } \\
\text { data from }\end{array}$ & SEC data from \\
\hline Island & Mellum & $\begin{array}{c}53^{\circ} 43^{\prime} \mathrm{N}, 8^{\circ} 08^{\prime} \\
E\end{array}$ & 38 & 23 & 15 & unpublished & unpublished \\
\hline \multirow{4}{*}{$\begin{array}{l}\text { Main- } \\
\text { land }\end{array}$} & $\begin{array}{c}\text { LS, } \\
\text { Leybucht }\end{array}$ & $\begin{array}{c}53^{\circ} 32^{\prime} N, 7^{\circ} 07^{\prime} \\
E\end{array}$ & 8 & 1 & 7 & $\begin{array}{l}\text { Minden and } \\
\text { Kleyer (2011) }\end{array}$ & unpublished \\
\hline & $\begin{array}{c}\text { LS, } \\
\text { Norderland }\end{array}$ & $\begin{array}{c}53^{\circ} 40^{\prime} \mathrm{N}, 7^{\circ} 19^{\prime} \\
E\end{array}$ & 32 & 13 & 19 & $\begin{array}{l}\text { Minden and } \\
\text { Kleyer (2011) }\end{array}$ & unpublished \\
\hline & $\begin{array}{l}\text { LS, Jade } \\
\text { Bight }\end{array}$ & $\begin{array}{c}53^{\circ} 26^{\prime} N, 8^{\circ} 09^{\prime} \\
E\end{array}$ & 32 & 1 & 31 & $\begin{array}{l}\text { Minden and } \\
\text { Kleyer (2011) }\end{array}$ & unpublished \\
\hline & $\begin{array}{c}\text { Schleswig- } \\
\text { Holstein }\end{array}$ & $55-54^{\circ} \mathrm{N}, 8-9^{\circ} \mathrm{E}$ & 112 & 90 & 22 & $\begin{array}{c}\text { Granse et al., in } \\
\text { preparation }\end{array}$ & unpublished \\
\hline \multirow{3}{*}{ Hallig } & Langeness & $\begin{array}{c}54^{\circ} 38^{\prime} N, 8^{\circ} 36^{\prime} \\
E\end{array}$ & 57 & 57 & NA & unpublished & $\begin{array}{l}\text { Schindler et al. } \\
\qquad \begin{array}{l}\text { (2014); } \\
\text { Hache et al., } \\
\text { unpublished }\end{array}\end{array}$ \\
\hline & $\begin{array}{l}\text { Nordstran- } \\
\text { dischmoor }\end{array}$ & $\begin{array}{c}54^{\circ} 33^{\prime} N, 8^{\circ} 48^{\prime} \\
E\end{array}$ & 24 & 24 & NA & unpublished & $\begin{array}{c}\text { Schindler et al. } \\
\text { (2014) }\end{array}$ \\
\hline & Hooge & $\begin{array}{c}54^{\circ} 34^{\prime} N, 8^{\circ} 23^{\prime} \\
E\end{array}$ & 32 & 32 & NA & unpublished & $\begin{array}{c}\text { Schindler et al. } \\
\text { (2014) }\end{array}$ \\
\hline
\end{tabular}




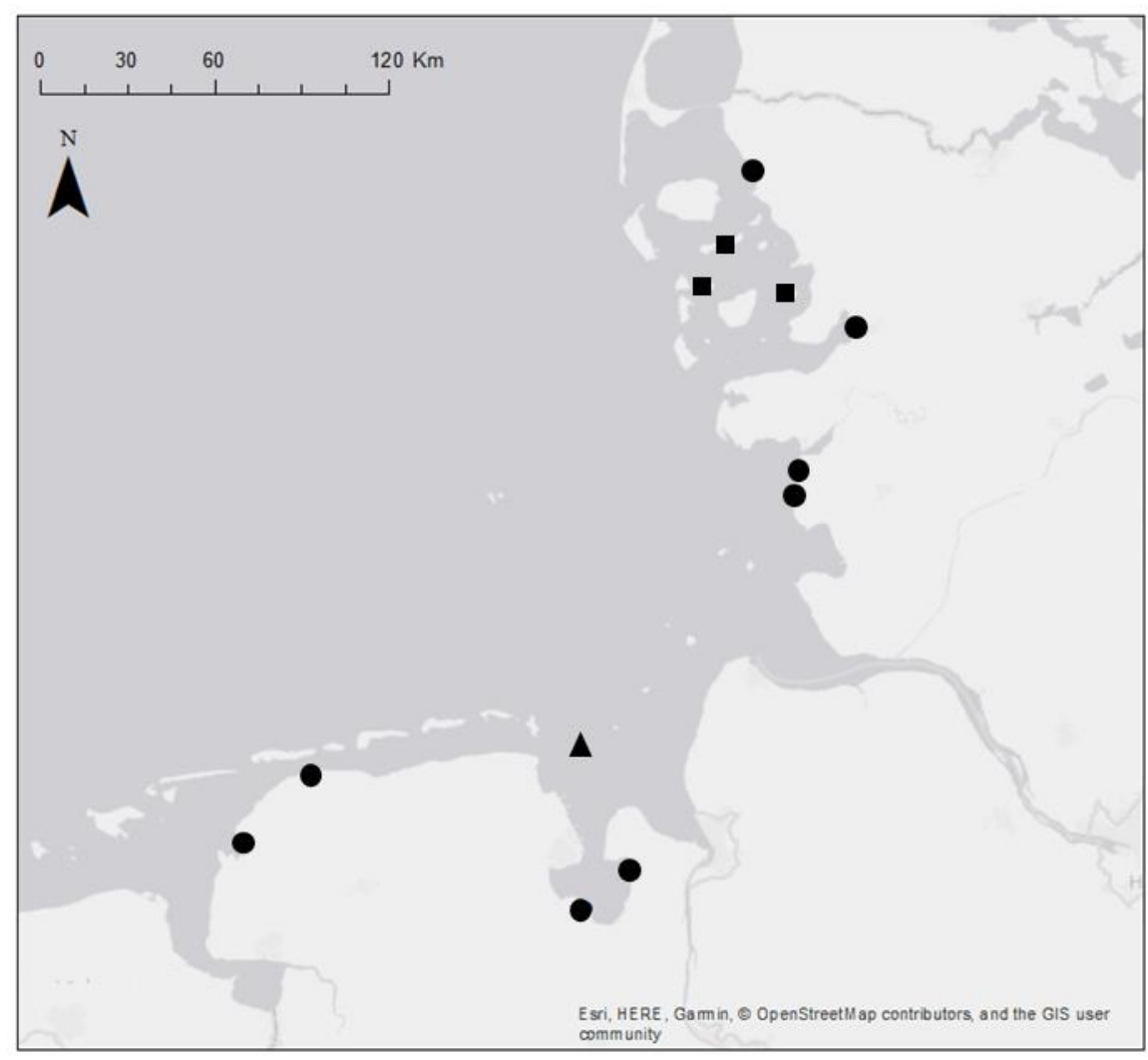

Figure 6-1: Map of the North Sea coast of Lower Saxony and Schleswig-Holstein including the islands and Halligen off the mainland coast. The island study area is indicated by a filled triangle, mainland study areas are indicated by filled circles, Hallig study areas are indicated by filled squares. (Single- column fitting image).

\subsubsection{Data collection}

\subsubsection{Surface elevation change}

Changes in SEC may be due to sedimentation, organic matter accumulation or decomposition, erosion, and soil compaction, among other factors. These processes cannot be clearly disentangled by many field measurement methods. We therefore used the neutral terms negative- or positive SEC. On mainland salt marshes in Lower Saxony and on island salt marshes, the average annual SEC was derived from measurements using sedimentation-erosion bars (Nolte et al. 2013a), the former over a period of 3 years (2006 to 2009), and the latter a period of 6 years (2006 to 2012). On mainland salt marshes in Schleswig-Holstein, the elevation above sea level was measured from 1987 to 1989 and again from 2017 to 2018 with an optical (Pentax AL-M5c) or automatic (Spectra Precision Laser Level LL500) leveling instrument, respectively, and the yearly SEC was calculated. On Hallig salt marshes, SEC data was derived from sediment traps established between 2010 and 2013 on Langeness, Hooge, and Nordstrandischmoor, and from 2016 to 2017 on Langeness. Data on SEC and species 
composition were recorded at the same plots. Only on the Halligen were SEC data from 2010 to 2013 manually extrapolated to the plots in which the vegetation was recorded, based on a detailed sedimentation map from Schindler et al. (2014b).

\subsubsection{Plant functional traits}

The functional trait composition of the vegetation served as predictor variables. Species composition and frequency were recorded at the plots on a $1 \mathrm{~m} \times 1 \mathrm{~m}$ square. Species nomenclature followed Jäger (2016). Canopy height (the distance between the highest fully grown leaf and the soil surface; $\mathrm{cm}$ ), plant stem biomass ( $\mathrm{mg})$, plant leaf biomass ( $\mathrm{mg}$ ), leaf area $\left(\mathrm{mm}^{2}\right)$, and specific stem length (length of a stem divided by its mass; $\mathrm{mm} / \mathrm{mg}$ ) were used to describe the above-ground features of the vegetation. Specific stem length describes stem stiffness, since stiff stems require denser, more lignified structures than flexible stems and therefore express greater masses per unit length. Below-ground biomass (mg), consisting of roots and rhizome biomass, and specific root length (length of a root divided by its mass; $\mathrm{mm} / \mathrm{mg}$ ) were used to describe below-ground features. All traits of the salt marsh species were collected from randomly chosen individuals in the field following the standards described in Knevel et al. (2005), Pérez-Harguindeguy et al. (2013), and Kleyer et al. (2019), albeit not from all plots. Most of the plants were collected on mainland and island plots in Lower Saxony (Mellum, Spiekeroog, Leybucht, Norderland, Jade Bight). In the other cases we assumed that, due to the regional proximity of all plots and their similar environmental regime, traits collected from populations in Lower Saxony could also be assigned to populations from SchleswigHolstein.

On the Halligen, plant height and above-ground biomass become decreased by grazing (Davidson et al. 2017). The 'Hallig-Programm', a state program to support Hallig farmers while promoting nature conservation, restricts the number of livestock units per hectare (LSU) to approx. $1 \mathrm{LSU}^{*} \mathrm{ha}^{-1}$, so that the grazing intensity varied mainly with the number of grazing days, rather than herd sizes. To account for the local biomass removal through cattle and sheep, sizerelated trait values were adjusted according to management intensities, data that was available from interviews with farmers and other land users. Plots were grouped according to the recorded grazing intensity into three groups: ungrazed, medium grazing intensity (< 300 grazing days*LSU), and high grazing intensity (> 300 grazing days*LSU). Based on our own observations, the canopy height of plants occurring in plots with medium and high grazing intensity was deliberately adjusted to $14 \pm 0.5 \mathrm{~cm}$ and $7 \pm 0.5 \mathrm{~cm}$, respectively. Subsequently, stem and leaf 
biomass were reduced in equal proportions. Below-ground traits are not strongly altered by grazing, thus local adjustment was not required.

\subsubsection{Distance to marsh edge}

As an abiotic factor for the analysis, plot distances to marsh edge $(\mathrm{m})$ were established from aerial images.

\subsubsection{Statistical analysis}

The annual SEC of the study areas were compared using an ANOVA and a Tuckey-HSD post-hoc test.

For further analysis, we subdivided the dataset according to salt marsh type and to plots experiencing either positive or negative SEC (cf. Table 6-1).

We used multiple linear regression to determine the effect on positive and negative SEC of the distance to the marsh edge, as well as of vegetation roughness and plant anchoring capacity. To meet the model's assumptions, we chose appropriate transformations using the 'bestNormalize' package (Peterson 2017) (see Table 6-2 for the transformations applied).

Highly correlated plant traits were aggregated based on a principal-component analysis (PCA; see Table 6-2 and Appendix Figure 6-5 and Figure 6-6), to avoid multicollinearity effects on the performance of individual predictors in the regression model. The above-ground traits leaf area (LA), leaf biomass (LBM), stem biomass (SBM), and specific stem length were aggregated to 'shoot mass' using the scores of the first PCA axis, which explained $66 \%$ of the variation of the four variables. LA, LBM, and SBM were positively correlated, and SSL was negatively correlated with the first PCA axis. Thus, increasing PCA 'shoot mass' scores indicate increasing vegetation roughness (scores ranged between +3.06 and -2.27 ; see Appendix Table 6-5 and Figure 6-5). Plants with high 'shoot mass' scores were Limonium vulgare (Mill.), Aster tripolium (L.), Spartina anglica (C.E. Hubb.), Atriplex prostrata (D.C.), and Halimione portulacoides (L.). Th e anchoring capacity was described by the scores of the first axis of a PCA using below-ground biomass (BIBM) and specific root length (SRL), which explained $67 \%$ of the variation of the correlated variables (scores ranged between +2.09 and -2.01; see Appendix Table 6-6 and Figure 6-6). Increasing BIBM and decreasing SRL values translate to increasing PCA 'anchoring' scores. Plants associated with high anchoring capacity are Plantago maritima (L.), Halimione portulacoides (L.), and Atriplex littoralis (L.). The trait canopy height was only marginally 
correlated with the other traits and thus satisfied the assumption of independence and entered the statistical analysis directly, i.e. without aggregation.

For each plot, the community-weighted mean trait (CWM; i.e. the average trait value or aggregated score of all species co-occurring in a plot, weighted by their abundance) were calculated (see Garnier et al. (2007)).

Table 6-2: Dependent and independent (biotic and abiotic) variables. Given are the plant functional traits, the corresponding abbreviations used in this study, as well as the aggregated variables based on a principle-component analysis (if traits were correlated) that are included in the multiple linear regression. Given are also minimum and maximum values, units and applied transformations; ORQ, ordered quantile normalization transformation.

\begin{tabular}{|c|c|c|c|c|c|c|c|}
\hline Factor & Trait & Abbrev & $\begin{array}{l}\text { Aggregated } \\
\text { variable }\end{array}$ & Min & Max & Unit & Transf \\
\hline \multirow[b]{2}{*}{ dependent } & positive SEC & PSEC & positive SEC & 0.0 & 26.7 & $\mathrm{~mm}$ & - \\
\hline & negative SEC & NSEC & $\begin{array}{l}\text { negative } \\
\text { SEC }\end{array}$ & -0.1 & -41.3 & $\mathrm{~mm}$ & - \\
\hline \multirow{5}{*}{$\begin{array}{l}\text { independent, } \\
\text { biotic, } \\
\text { above- } \\
\text { ground }\end{array}$} & $\begin{array}{l}\text { canopy } \\
\text { height }\end{array}$ & $\mathrm{CH}$ & $\begin{array}{l}\text { canopy } \\
\text { height }\end{array}$ & 0.6 & 70.1 & $\mathrm{~cm}$ & ORQ \\
\hline & $\begin{array}{r}\text { plant stem } \\
\text { biomass }\end{array}$ & SBM & & 22.6 & 17259.2 & $\mathrm{mg}$ & boxcox \\
\hline & $\begin{array}{l}\text { plant leaf } \\
\text { biomass }\end{array}$ & LBM & 'shoot mass' & 7.1 & 4172.2 & $\mathrm{mg}$ & $\arcsin$ \\
\hline & leaf area & LA & & 16.9 & 2071.6 & $\mathrm{~mm}^{2}$ & ORQ \\
\hline & $\begin{array}{l}\text { specific stem } \\
\text { length }\end{array}$ & SSL & & 0.5 & 9.3 & $\mathrm{~mm} / \mathrm{mg}$ & ORQ \\
\hline \multirow{2}{*}{$\begin{array}{l}\text { independent, } \\
\text { biotic, } \\
\text { below- } \\
\text { ground }\end{array}$} & $\begin{array}{c}\text { below-ground } \\
\text { biomass }\end{array}$ & BIBM & 'anchoring' & 15 & 24799 & $\mathrm{mg}$ & ORQ \\
\hline & $\begin{array}{l}\text { specific root } \\
\text { length }\end{array}$ & SRL & & 0.4 & 105.9 & $\mathrm{~mm} / \mathrm{mg}$ & ORQ \\
\hline $\begin{array}{l}\text { independent, } \\
\text { abiotic }\end{array}$ & $\begin{array}{l}\text { distance to } \\
\text { marsh edge }\end{array}$ & dist & $\begin{array}{l}\text { distance to } \\
\text { marsh } \\
\text { edge }\end{array}$ & 0 & 779 & $\mathrm{~m}$ & $\begin{array}{r}\text { boxcox/ } \\
\text { ORQ }\end{array}$ \\
\hline
\end{tabular}


To select the best environmental predictor for positive and negative SEC, a stepwise variable selection was used to find the best performing model with the lowest AIC (Akaike Information Criterion). All statistical analyses were conducted in the computing environment $\mathrm{R}$ (version 3.5.2).

\subsection{Results}

The mean SECs were significantly different between the study areas (ANOVA results: $F(3,331)=$ 40.44, $p<0.001$ ) (Figure 6-2). On the island, representing the most natural marsh in the dataset, SEC varied from $17 \mathrm{~mm} / \mathrm{a}$ to $-22 \mathrm{~mm} / \mathrm{a}$, with a mean of $2 \mathrm{~mm} / \mathrm{a}$. On mainland marshes of Schleswig-Holstein, mainly positive SEC was found, ranging between $19 \mathrm{~mm} / \mathrm{a}$ and $-12 \mathrm{~mm} / \mathrm{a}$, with a mean of $4 \mathrm{~mm} / \mathrm{a}$. In contrast, mainland marshes of Lower Saxony mostly showed negative SEC, with a wide range from $27 \mathrm{~mm} / \mathrm{a}$ to $-41 \mathrm{~mm} / \mathrm{a}$, and a mean of $-6 \mathrm{~mm} / \mathrm{a}$. Note that the Schleswig-Holstein time series spanned 30 years, while the series from Lower Saxony spanned only 3 years. Plots on the Halligen salt marsh showed the smallest range of SEC, from 0 $\mathrm{mm} / \mathrm{a}$ to $9 \mathrm{~mm} / \mathrm{a}$ and a mean of $2 \mathrm{~mm} / \mathrm{a}$. 


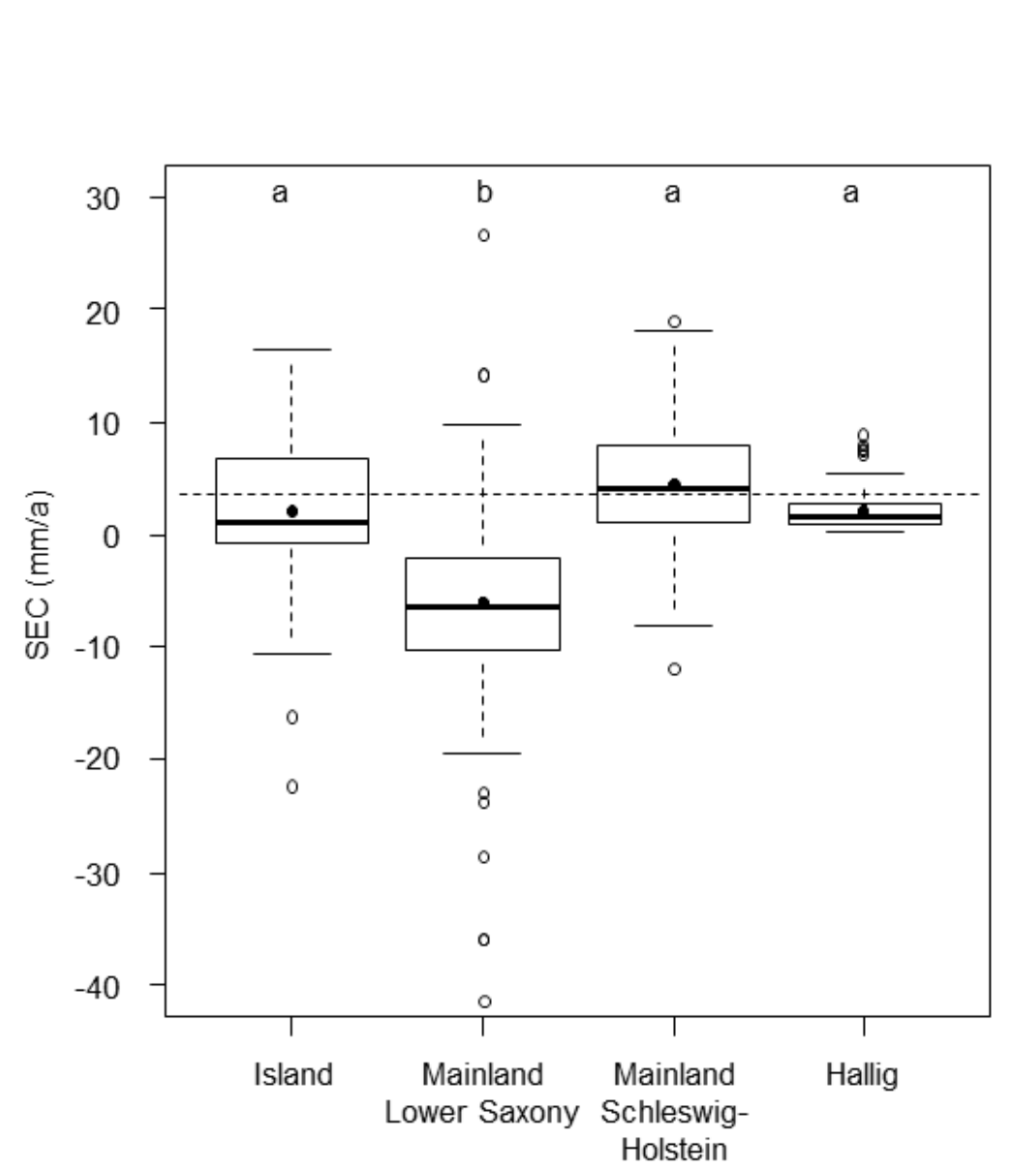

Figure 6-2: Annual SEC of the study areas island, mainland (due to regional differences divided between Lower Saxony and Schleswig-Holstein), and Hallig. The boxes represent the interquartile ranges, the whiskers the upper and lower fences, the circles outliers, the thick lines indicate the medians, and the solid dots the means. The dashed line shows the annual mean sea-level rise $(3.6 \mathrm{~mm} / \mathrm{a})$ in the German Bight from 1971 to 2008 according to Wahl et al. (2011). Letters indicate significant differences among study areas according to a Tukey-HSD post hoc test. (Single-column fitted image).

\subsubsection{Positive SEC}

On the island marshes (Figure 6-3a and Table 6-3), a positive SEC correlated non-linearly with distance to the marsh edge, with a peak at an intermediate distance and strongly decreasing values near to, and far from, the edge. Plant canopy height showed an almost bathtub-shaped relationship, with the highest positive SEC on plots with high canopy heights. On mainland salt marshes (Figure 6-3b and Table 6-3), a positive SEC showed a bathtub-shaped relationship with distance to the marsh edge, with the highest values close to the edge. It also showed a humpshaped relationship with 'shoot mass', with the highest positive SEC values at intermediate to higher 'shoot mass' values. On Hallig salt marshes (Figure 6-3c and Table 6-3), we found a linear relationship with distance to the marsh edge and a bathtub-shaped relationship with canopy height. A positive SEC was highest on plots close to the marsh edge that had high vegetation. 

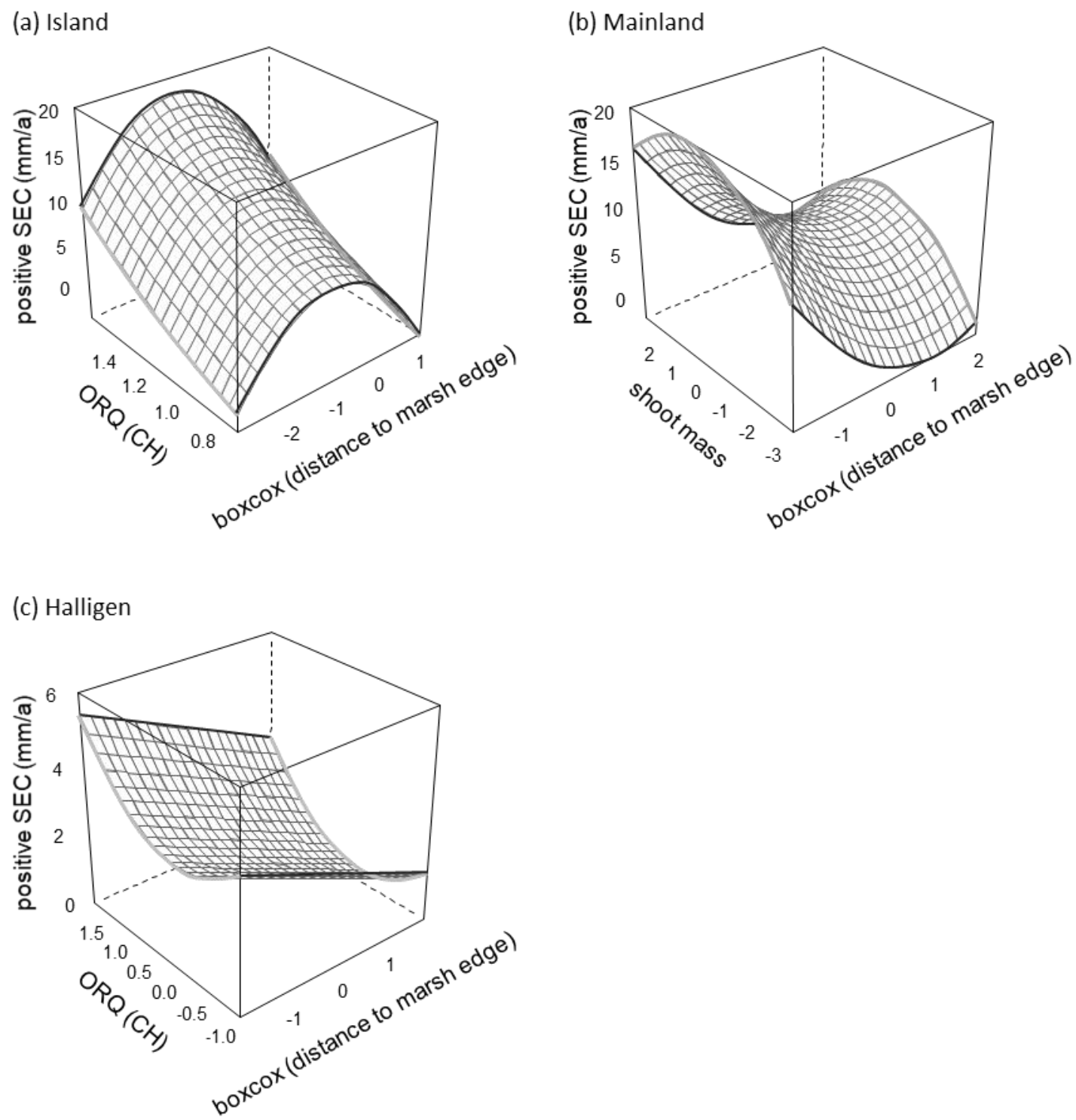

Figure 6-3: Factors explaining positive SEC on salt marshes of (a) island ( $n=23)$, (b) mainland ( $n=105)$, and (c) Halligen ( $n=113$ ). Distance to marsh edge is boxcox transformed, where a value of -1 corresponds to $77 \mathrm{~m}$, a value of +1 corresponds to $431 \mathrm{~m}$. Canopy height $(\mathrm{CH})$ is $\mathrm{ORQ}$ transformed, where a value of -1 corresponds to $6.8 \mathrm{~cm}$, a value of +1 corresponds to $28.2 \mathrm{~cm}$. 'Shoot mass' values are the scores of the first axis from the PCA (see Appendix Figure 6-5) using LA, LBM, SBM, and SSL. (2-column fitted image). 
Table 6-3: Results of the multiple linear regression model and stepwise variable selection for positive SEC on island marshes, mainland marshes and Hallig marshes. Given are estimates, standard error, $t-$ values, and significance levels. Significant $p$-values at $\alpha<0.05$ are shown in bold type. In addition, measures of model performance are shown.

Estimate $\quad$ SE $\quad t \quad p$

Island marshes
intercept
distance to marsh edge
distance to marsh edge^ 2
$\mathrm{CH}^{\wedge} 2$
Adj. $R^{2}=0.27 ; F(3,19)=3.72, p=0.03$

\section{Mainland marshes}

$\begin{array}{llllll}\text { intercept } & 8.79 & 0.65 & 13.52 & <0.001 & * * * \\ \text { distance to marsh edge } & -3.46 & 0.66 & -5.26 & <0.001 & * * * \\ \text { distance to marsh edge^2 } & 1.54 & 0.43 & 3.57 & <0.001 * * * \\ \text { shoot mass } & 0.65 & 0.39 & 1.66 & 0.100 & \\ \text { shoot mass^2 } & -0.71 & 0.17 & -4.20 & <0.001 * * * \\ \mathrm{CH} & -0.71 & 0.51 & -1.39 & 0.168\end{array}$

Adj. $R^{2}=0.44 ; F(5,99)=17.0, p<0.001$

\section{Hallig marshes}

intercept

1.87

2.21

0.85

0.408

$-4.64$

1.48

$-3.14$

$0.005 * *$

$-2.37$

0.90

$-2.64$

$0.016 *$

5.59

2.21

2.54

$0.020 *$

intercept

distance to marsh edge

1.70

0.15

11.669

0.14

$-5.016$

$\mathrm{CH}^{\wedge} 2$

$-0.68$

0.19

4.195

$\begin{array}{ll}<0.001 & * * * \\ <0.001 & * * * \\ <0.001 & * * *\end{array}$

Adj. $R^{2}=0.29 ; F(2,110)=23.7, p<0.001$

\subsubsection{Negative SEC}

On island marshes (Figure 6-4a and Table 6-4), negative SEC corresponded linearly to distance to the marsh edge, with high negative SEC close to the edge, often due to nearby meandering creeks. Negative SEC decreased linearly with increasing anchoring values. High negative SEC was found on pioneer zones with abundant Salicornia spp., whereas pioneer zones with Spartina anglica were most often characterized by sedimentation (see above). On mainland marshes (Figure 6-4b and Table 6-4), negative SEC showed a hump-shaped relationship with 
distance to the marsh edge, with the highest values close to the edge. It also showed a linear relationship with anchoring, with the highest values in plots with high anchoring values.

(a) Island

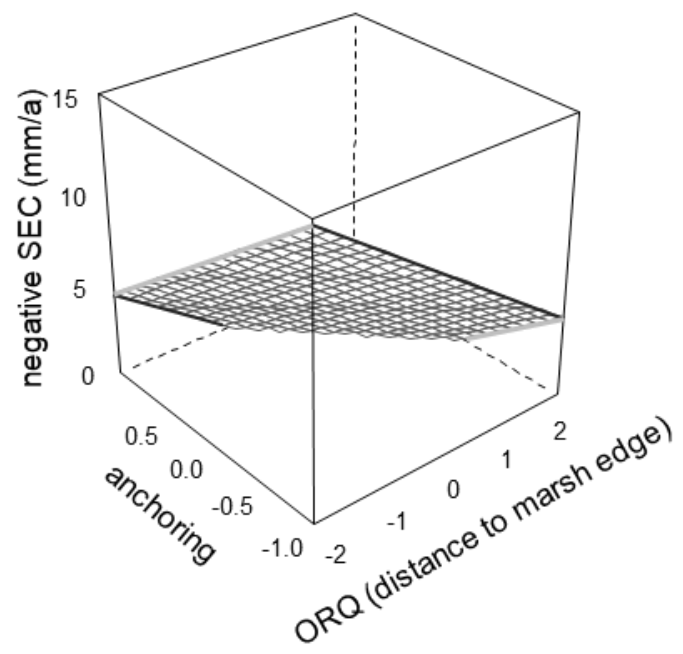

(b) Mainland

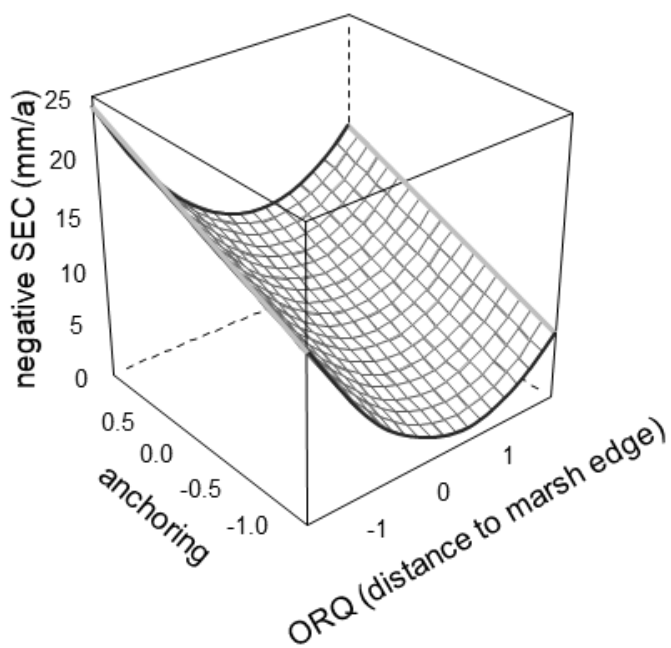

Figure 6-4: Factors explaining negative SEC on salt marshes of $(a)$ island $(n=15)$ and $(b)$ mainland $(n=79)$ locations. Distance to the marsh edge is ORQ transformed, where a value of -1 corresponds to $125 \mathrm{~m}$, a value of +1 corresponds to $390 \mathrm{~m}$. 'Anchoring' values are the scores of the first axis from the PCA (see Appendix Figure 6-6) using BIBM and SRL. (2-column fitted image).

Table 6-4: Results of the multiple linear regression model for negative SEC on island- and mainland marshes. Given are estimates, standard errors, $t$-values, and significance levels. Significant $p$-values at $\alpha$ $<0.05$ are shown in bold type. In addition, measures of model performance are shown.

\section{Estimate}

4.16

$-2.41$

$-4.83$

\section{Island marshes}

intercept

distance to marsh edge

anchoring

Adj. $R^{2}=0.66 ; F(2,12)=14,64, p<0.001$

\section{Mainland marshes}

\section{intercept}

distance to marsh edge

distance to marsh edge ${ }^{\wedge} 2$

anchoring

\subsection{6}

$-2.65$

2.62

3.98
SE

1.06

0.96

1.76 $t$

p

Adj. $R^{2}=0.27 ; F(3,75)=10.62, p<0.001$ 


\subsection{Discussion}

The German North Sea coast is sensitive to changes in sea level, as isostatic land subsidence adds to the global rise in sea level. In addition, it is a meso- to macrotidal region in which mean high water - determining the border between tidal flat and salt marsh - is expected to rise more than is mean sea level (Balke et al. 2016). To keep current marsh distribution in place, marsh surface elevation must rise via sedimentation. The interquartile range of all SEC data range from -1.0 to $+4.3 \mathrm{~mm} / \mathrm{a}$. Assuming similar SEC rates in the future, these observations question the ability of the marshes to accrete sufficiently to follow recent SLR (Wahl et al. 2011) and predicted, future SLR (IPCC, in press).

Our results demonstrate considerable total variation in changes in surface elevation across all plots, ranging from -41.3 to $+26.7 \mathrm{~mm} / \mathrm{a}$. Even at the same location, if vegetation is scattered, surface elevation may change from positive to negative SEC, and vice versa, from year to year (pers. observation). This is partly due to the strong stochastic contribution of storm surges. In contrast to the deterministic diurnal tidal regime, a single storm surge can deposit or resuspend large amounts of sediment or organic material. The SEC distribution can vary strongly, depending on the coincidence of high water, wave energy, water current, and wind direction (Hache et al. 2019). Thus, marshes could benefit from high sedimentation in one year, but low sedimentation in another year. In addition, sedimentation depends on the concentration of suspended matter in the sea water (French 2006), a parameter we could not include in the models. Spatiotemporal stochasticity in flooding intensity and sediment transport may explain the fairly moderate goodness-of-fit measures of our models, as shown in with other studies. For instance, Boorman et al. (1998) found a correlation between vegetation height and surface accretion on one salt marsh, but none on another marsh. Nevertheless, our results demonstrate that SEC is related to the distance to the marsh edge, and to the functional properties of the marsh vegetation. Vegetation roughness enhances sedimentation in a nonlinear way and therefore supports surface elevation gain. Vegetation anchoring can reduce erosion and therefore lowers elevation loss.

\subsubsection{Biogeomorphic feedback}

The distance to the marsh edge was the main explanatory variable for positive SEC in all three salt marsh types, with a strong, (non-) linear decrease in SEC values further inland. This is in accordance with previous work showing an increase in elevation (Suchrow et al. 2012) and in 
sedimentation rates in particular (Schindler et al. 2014b). The main reason for this may be a strong decrease in wave energy and current strength between mud flats and salt marshes where dense vegetation at the boundary dissipates energy (Möller and Spencer 2002). In addition, inundation times are longer, and exposure to settling sediment is favored at lower elevations close to the marsh edge (Temmerman et al. 2003b). During shallow inundation on high salt marshes, water and sediment are often mainly supplied via the creek system, and subsequently deposited close to the source and along the creek levees (Temmerman et al. 2005b). This would make distance to the creeks more important than distance to the marsh edge. On the island, we found a strong decrease in average SEC close to the marsh edge. This can be attributed to a highly dynamic SEC close to the marsh edge, often oscillating between erosion and sedimentation from year to year (Kleyer et al. 2014).

\subsubsection{Positive SEC explained by functional traits}

Vegetation roughness significantly increased positive SEC in all three types of salt marsh. Roughness is a composite property determined by plant height, leaf size and biomass, and shoot biomass and stiffness, all of which may affect wave energy, water current, and sediment deposition.

The Hallig vegetation is characterised mainly by upper salt marsh communities, with varying grazing intensity. Strong grazing alters plant height, resulting in a low, homogeneous sward at the end of the vegetation period when the storm surge season starts. Ungrazed or less grazed areas may have a similar species composition as the grazed parts (mainly of the Juncus gerardiiFestuca rubra type), but with fully grown, often senescent stems. Thus, the main roughness indicator explaining sedimentation on Hallig salt marshes was plant canopy height. Other studies, however, found a significant impact of grazing on vegetation height on salt marshes, but no subsequent effect on sedimentation (Elschot et al. 2013; Nolte et al. 2013b). In contrast to the Halligen, sedimentation on the mainland coast was explained by 'shoot mass'. The mainland coast is characterized by more vegetation types, covering pioneer zone, lower salt marsh, and upper salt marsh; more varied micro-topography (i.e. beds and ditches, levees, dyke foot, hummocks), and different management strategies (from bare soil to grazed and natural conditions, see Suchrow et al. (2012) and Minden et al. (2012)). These diverse conditions are better reflected in the variation of 'shoot mass', the first principal component of leaf area, leaf biomass, stem biomass, and specific stem length. These components were found to be central organizing traits of herbaceous plant diversity of Northwest Europe (Kleyer et al. 2019). 
Specifically, the hump-shaped relationship, with the peak towards the higher 'shoot mass' values (Figure 6-4b), indicates that rigid shoots with high biomass enhance the capacity of the vegetation to trap sediments. Similar results were found by Bouma et al. (2010) in flume experiments for Puccinellia maritima, a plant with very flexible shoots, and Spartina anglica, characterised by rigid, erect stems. Vegetation community structure has been found to affect sedimentation, and the highest sedimentation rates coincide with maximum plant population densities and biomass during summer months (Pasternack and Brush 2002). Other findings showed that grazing induced a different vegetation structure, which then led to different sedimentation intensities (Andresen et al. 1990; Stock 2011).

Our findings reflect the concept of ecosystem engineers in salt marshes. Engineering species greatly influence geomorphic processes and modify their own environment by attenuating waves and currents, enhancing sedimentation, and stabilizing sediment. In salt marshes, a wellaccepted example for an engineering plant species is Spartina spp. (Christiansen et al. 2000; Neumeier and Ciavola 2004). In our study, stem traits of Spartina anglica indicated high stem stiffness (S. anglica SSL: $0.51 \mathrm{~mm} / \mathrm{mg}$; mean of all salt marsh species: $3.39 \mathrm{~mm} / \mathrm{mg}$ ) and high leaf area (S. anglica LA: $1380.2 \mathrm{~mm}^{2}$; mean of all salt marsh species: $514.19 \mathrm{~mm}^{2}$ ). The vegetation-directed sedimentation initiates elevation gain, which in turn feeds back on vegetation patterns (Nyman et al. 2006).

\subsubsection{Negative SEC explained by functional traits}

Vegetation also had an effect on negative SEC. Several studies have claimed that wetland vegetation can provide protection from erosion (Barbier et al. 2011; Danielsen et al. 2005; Kathiresan and Rajendran 2005). van Eerdt (1985) separated the effects of above- and belowground biomass on erosion resistance, and showed that it was solely below-ground biomass that prevents erosion. On island marshes, we found the lowest negative SEC in plots with low plant-root anchoring values, in line with the results of (van Eerdt 1985). The island displayed a natural salt marsh zonation on predominantly sandy soils, with an extended pioneer zone. Shifting meandering creeks were probably responsible for the erosion affecting plots located in their catchment area. The highest erosion occurred on almost bare sites with low cover of the small annual Salicornia spp., a plant with tiny roots, whereas adjacent patches with Spartina anglica still experienced sedimentation.

In contrast, on mainland marshes of Lower Saxony, large negative SEC values occurred in plots where plants showed high anchoring values. Here, we had established plots mainly on lower 
$(n=28)$ and upper marshes $(n=25)$, because pioneer zones were often small and too muddy and dangerous to access. Coast-wide habitat maps (Nationalpark Wattenmeer 2004) show that some marshes occur in typical zonation, others with the upper marsh in the seaward direction and lower marsh in the landward direction. The latter zonation may have arisen from old land reclamation schemes in which rectangular groyne fields were constructed on the mudflats to trap sediment, leading to high accretion rates and thus upper marsh communities near the seaward border of the groyne fields. In areas exposed to high wave energy, ridges with upper marsh vegetation developed close to the marsh edge (e.g., the eastern side of the Jade Bight). This diverse pattern close to the marsh edge may explain why high negative SEC values were found on plots dominated by perennial plants featuring high root biomass, such as Limonium vulgare, Elytrigia atherica or Atriplex portulacoides. The time series of these plots comprised three years that saw particularly strong storm surges as compared to other years (storms “Britta", Nov. 1" $1^{\text {st }}, 2006$, water level +5.5 m near Emden; “Tilo", Nov. $7^{\text {th }}, 2007$, water level +4.4 $m$ near Cuxhaven). If we could have covered a period of 30 years, as in Schleswig-Holstein, a positive instead of negative SEC would have been likely. Nevertheless, this specific observation period made it possible to demonstrate that even high below-ground biomass can support negative SEC, probably because it coincides with high above-ground roughness that facilitates wave breaking during strong flood events (Temmerman et al. 2007).

\subsection{Conclusion}

Our study comprised an unprecedented dataset of surface elevation change records on German North Sea salt marshes that can be explained by abiotic and biotic factors. With the notable exception of Lower Saxony mainland plots that were studied only for three years, and coinciding with a period of strong storm surges, the mean annual accretion values were lower or close to recent annual sea level rise (Wahl et al. 2011). Whether marsh accretion can keep pace with increasing sea level rise in the future remains an open question. To enable predictions of future accretion, a better understanding of the amounts and pathways of sediment erosion and mobilization, transportation, and deposition in relation to stochastic storm surges and deterministic diurnal tidal flooding is necessary. In addition to modelling, a network of permanent stations to record yearly surface elevation changes should be installed, 
similar to the state-managed network of water-level gauges. Yearly records are desirable, because the available 6-year time series from Mellum island revealed an interannual variation in sedimentation (Kleyer et al. 2019) that made it possible to disentangle the effects of storm surges and diurnal tidal floods.

Positive and negative SEC corresponded to plant traits and the distance to the marsh edge. We conclude that salt marsh vegetation is most likely well suited to modify and control SEC in response to sea-level rise or tidal forces. Using traits instead of species identity increased predictability, as studies using species composition as the predictor did not find conclusive effects on SEC (Brown 1998; Silva et al. 2009). Empirically measured plant community mean traits indicate vegetation roughness and anchoring capability in a more mechanistic way than the vegetation-roughness classification applied for the widely used Gauckler-Manning-Strickler formulas in hydraulics (Chow 1959). In conjunction with the traits of salt marsh plants (Appendix Table 6-5 and Table 6-6) and maps of salt marsh communities available for the Wadden Sea region, our predictive functions allow the modelling of spatially explicit SECs to manage accretion in salt marshes. On the Halligen, for instance, a low plant height near the marsh edge and a higher height farther away would favor the distribution of sediment from the marsh edge to the hinterland. This could be deliberately steered by the grazing intensity. The different shapes of our response functions from Hallig, mainland, and island plots indicate that future studies should take into account differences in salt marsh genesis and land use. 


\subsection{Appendix}

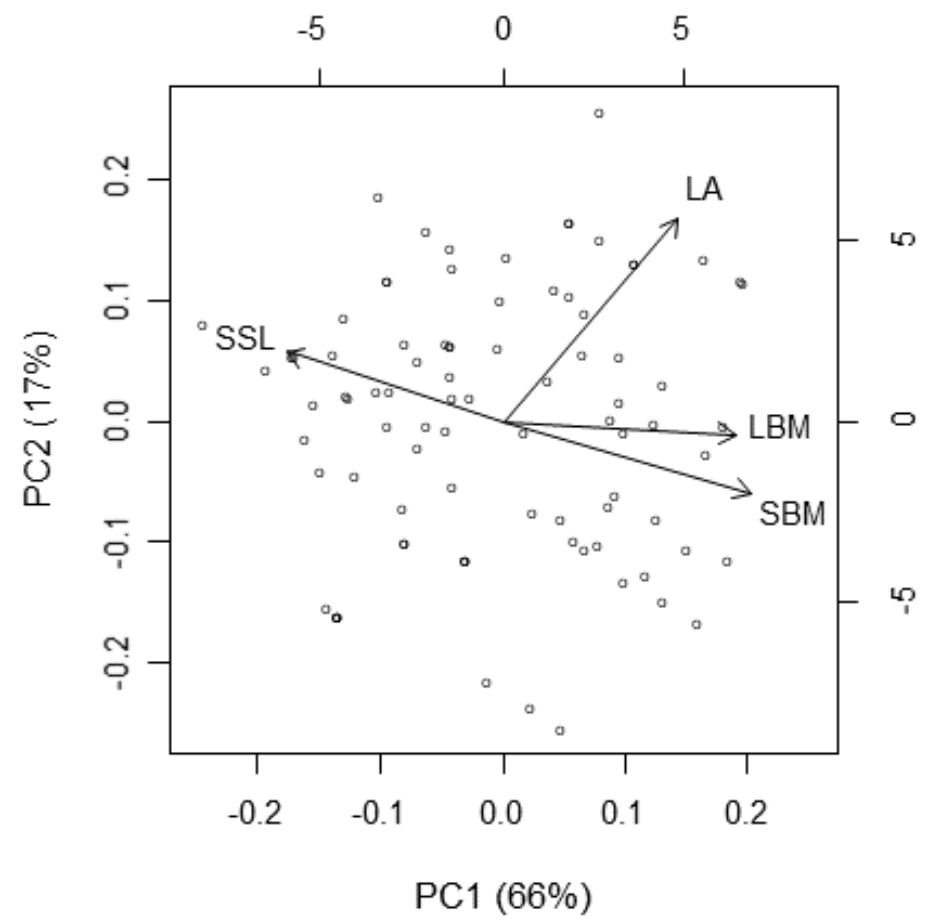

Figure 6-5: Two-dimensional illustrations of the standard PCA ordination results of plots that experienced positive SEC. Arrows indicate the direction of loading for each trait. The scores of the first axis are included in the model as 'shoot mass'. LA, leaf area; LBM, leaf biomass; SBM stem biomass; SSL, specific stem length. (Single-column fitted image). 
Table 6-5: Functional traits and the aggregated variable 'shoot mass' of species growing on plots that experienced positive SEC. CH, canopy height ( $\mathrm{cm})$; LA, leaf area ( $\mathrm{mm} 2)$; LBM, leaf biomass $(\mathrm{mg})$, SBM, stem biomass (mg); SSL, specific stem length $(\mathrm{mm} / \mathrm{mg})$.

\begin{tabular}{|c|c|c|c|c|c|c|}
\hline Species & $\begin{array}{l}\text { 'shoot } \\
\text { mass' }\end{array}$ & $\mathrm{CH}$ & LA & LBM & SBM & SSL \\
\hline Agrostis stolonifera (L.) & $-0,64$ & 31,08 & 207,70 & 50,17 & 233,36 & 3,57 \\
\hline Armeria maritima (Mill.) Willd. & $-0,38$ & 5,88 & 68,30 & 353,11 & 197,30 & 3,94 \\
\hline Artemisia maritima (L.) & 1,87 & 33,25 & 208,50 & 675,61 & 1765,65 & 0,66 \\
\hline Aster tripolium (L.) & 2,89 & 60,84 & 663,90 & 1174,96 & 10074,12 & 0,60 \\
\hline Atriplex littoralis (L.) & 2,37 & 70,13 & 494,60 & 509,12 & 17259,26 & 0,69 \\
\hline Atriplex prostrata (D.C.) & 2,64 & 42,07 & 815,80 & 2354,47 & 9519,62 & 1,51 \\
\hline Cochlearia danica (L.) & $-0,52$ & 7,40 & 127,70 & 145,27 & 374,18 & 4,71 \\
\hline Elytrigia atherica (Link.) & 1,41 & 38,90 & 773,60 & 314,35 & 1036,67 & 0,98 \\
\hline Elytrigia repens (L.) Gould & 1,05 & 51,25 & 904,60 & 241,33 & 720,52 & 1,88 \\
\hline Festuca rubra (L.) & $-0,96$ & 37,42 & 126,10 & 228,60 & 144,79 & 6,86 \\
\hline $\begin{array}{c}\text { Festuca rubra litoralis (G.F.W. } \\
\text { Meyer) }\end{array}$ & $-2,27$ & 26,83 & 94,00 & 28,74 & 91,32 & 7,21 \\
\hline Glaux maritima (L.) & $-1,97$ & 8,10 & 17,00 & 67,14 & 72,94 & 4,48 \\
\hline Halimione portulacoides (L.) Aellen & 2,51 & 28,71 & 186,20 & 2869,74 & 9183,15 & 0,95 \\
\hline Juncus gerardii (Lois.) & $-1,36$ & 24,72 & 139,40 & 54,96 & 129,48 & 5,04 \\
\hline Limonium vulgare (Mill.) & 3,06 & 14,51 & 1681,30 & 4172,17 & 1193,61 & 0,75 \\
\hline Lolium perenne (L.) & 0,35 & 34,18 & 461,60 & 99,19 & 620,65 & 2,16 \\
\hline Lotus corniculatus (L.) & $-0,56$ & 27,80 & 374,60 & 116,48 & 135,99 & 4,29 \\
\hline Plantago maritima (L.) & 1,73 & 13,15 & 1401,70 & 1239,13 & 688,29 & 3,08 \\
\hline Poa pratensis (L.) & $-0,52$ & 31,09 & 285,70 & 86,00 & 213,71 & 4,42 \\
\hline Potentilla anserina (L.) Rydb. & $-0,54$ & 19,94 & 773,30 & 386,61 & 209,64 & 9,28 \\
\hline Puccinellia maritima (Huds.) Parl. & $-1,17$ & 28,82 & 76,20 & 62,52 & 209,93 & 4,63 \\
\hline Salicornia europaea (L.) & 1,11 & 17,24 & 162,40 & 799,62 & 719,96 & 1,49 \\
\hline Spartina anglica (C.E. Hubb.) & 2,83 & 44,27 & 1380,20 & 764,80 & 1425,34 & 0,51 \\
\hline Spergularia maritima (L.) & $-1,15$ & 26,75 & 46,55 & 126,09 & 548,28 & 1,93 \\
\hline Spergularia media (L.) C. Presl & $-0,58$ & 7,72 & 446,10 & 173,81 & 261,52 & 7,08 \\
\hline Suaeda maritima (L.) Dumort. & 0,80 & 25,02 & 32,00 & 494,34 & 1602,82 & 1,27 \\
\hline Trifolium repens (L.) & $-1,34$ & 10,96 & 474,60 & 56,77 & 75,27 & 5,82 \\
\hline Triglochin maritimum (L.) & 1,11 & 22,57 & 931,50 & 574,31 & 380,47 & 2,25 \\
\hline Vicia cracca (L.) & $-0,87$ & 27,50 & 142,10 & 162,58 & 112,19 & 4,51 \\
\hline
\end{tabular}




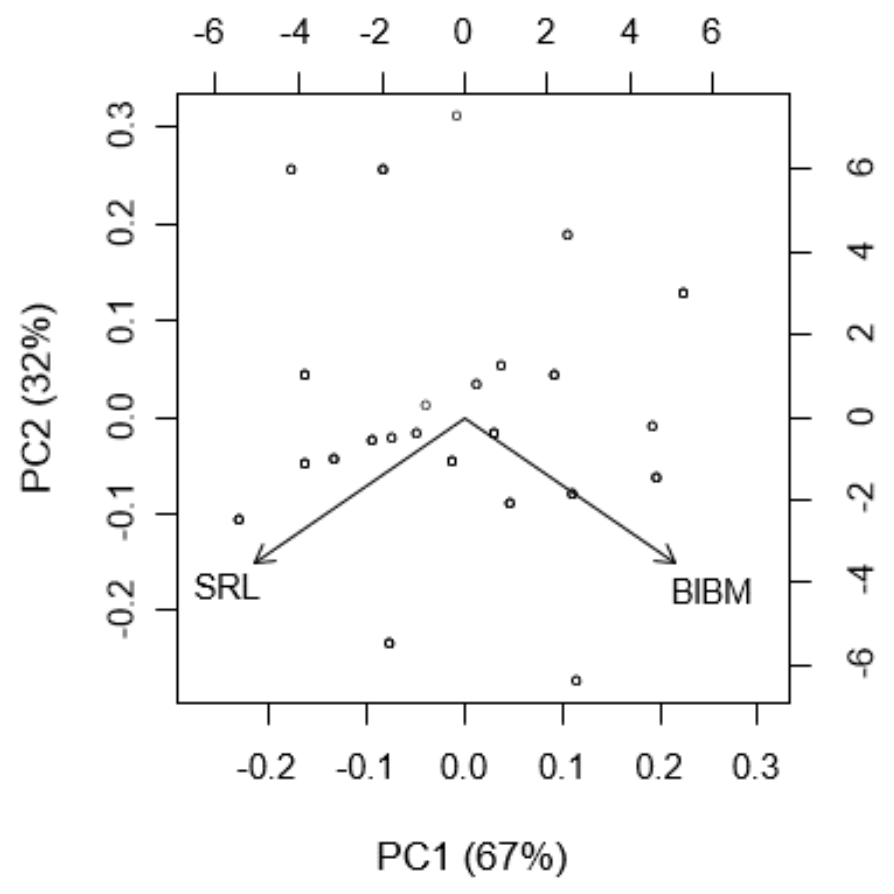

Figure 6-6: Two-dimensional illustration of the standard PCA ordination results of plots that experienced negative SEC. Arrows indicate the direction of loading for each trait. The scores of the first axis are included in the model as 'anchoring'. BIBM, below-ground biomass; SRL, specific root length. (Singlecolumn fitted image). 
Table 6-6: Functional traits and the aggregated variable 'anchoring' of species growing on plots that experienced negative SEC. BIBM, below-ground biomass ( $\mathrm{mg})$; SRL, specific root length $(\mathrm{mm} / \mathrm{mg}$ ).

\begin{tabular}{|c|c|c|c|}
\hline Species & 'anchoring' & BIBM & SRL \\
\hline Agrostis stolonifera (L.) & $-2,01$ & 79,44 & 105,87 \\
\hline Armeria maritima (Mill.) Willd. & $-0,37$ & 279,73 & 27,41 \\
\hline Artemesia maritima (L.) & 0,50 & 1528,13 & 17,75 \\
\hline Aster tripolium (L.) & 1,07 & 3168,29 & 15,21 \\
\hline Atriplex littoralis (L.) & 1,80 & 3377,80 & 10,63 \\
\hline Atriplex prostrata (D.C.) & $-0,03$ & 498,57 & 26,54 \\
\hline Cochlearia danica (L.) & 0,01 & 48,32 & 7,09 \\
\hline Elymus pycnanthus (Godr.) Melderis & 0,16 & 282,59 & 15,42 \\
\hline Elymus repens (L.) Gould & 0,42 & 316,63 & 15,10 \\
\hline Festuca rubra litoralis (G.F.W. Meyer) & $-1,40$ & 78,50 & 45,65 \\
\hline Glaux maritima (L.) & $-0,68$ & 40,44 & 13,39 \\
\hline Halimione portulacoides (L.) Aellen & 1,84 & 3908,03 & 11,03 \\
\hline Juncus geradii (Lois.) & 1,03 & 282,28 & 6,07 \\
\hline Leontodon autumnalis (L.) & $-0,28$ & 194,44 & 25,12 \\
\hline Limonium vulgare (Mill.) & 1,11 & 15173,65 & 28,53 \\
\hline Lolium perenne (L.) & $-0,60$ & 186,78 & 30,07 \\
\hline Plantago maritima (L.) & 2,09 & 2063,55 & 0,98 \\
\hline Puccinellia maritima (Huds.) Parl. & $-1,39$ & 153,43 & 56,98 \\
\hline Salicornia europaea (L.) & $-1,12$ & 165,33 & 53,24 \\
\hline Spartina anglica (C.E. Hubb.) & 0,34 & 758,58 & 16,58 \\
\hline Spergularia media (L.) C. Presl & $-0,62$ & 1477,38 & 69,23 \\
\hline Stellaria media (L.) Vill. & $-1,52$ & 14,96 & 17,40 \\
\hline Suaeda maritima (L.) Dumort. & $-0,77$ & 183,22 & 34,84 \\
\hline Trifolium maritimum (L.) & 0,90 & 967,68 & 12,97 \\
\hline
\end{tabular}

\section{Acknowledgement}

The study forms part of the joint research project "Real life coastal laboratory to develop shortand long term strategies for the protection and preservation of Schleswig-Holsteins Halligen 
(Living Coast Lab Hallig)" funded by the German Federal Ministry of Education and Research (BMBF, 03F0759D). We thank the landowners for their hospitality and for supporting the research project. 


\section{$7 \quad$ Implications for coastal protection}

\subsection{The main SPM transport mechanism issue}

Coastal protective measures such as dykes seawalls and embankments are widely perceived as the ultimate solution to combat flood risks in times of global change (Temmerman et al. 2013). Earlier studies even suggest that direct human modification, rather than sea-level rise, is by far the major cause of historical and contemporary coastal wetland loss (Kirwan and Megonigal 2013). Hence, the new findings from this work may contribute to estimating negative consequences of already undertaken and future interventions in coastal ecosystems. Conscious action and weighing benefits and risk factors, especially when interfering with an already unbalanced ecosystem like the Halligen, is a challenging task. An example in the Scheldt estuary, Belgium demonstrates how selective anthropogenic interventions in the ecosystem can influence vertical accretion rates by restoring a deeper estuarine habitat (polder) behind a natural marshland (Cox et al. 2006) and reveals the main mechanism issues for the Halligen to keep pace with SLR. This is done through controlled flooding and drainage of the polder via two dykes and a tidal gate. A higher ring dyke protects the settlements from storm surges, while the polder can be flooded in a controlled manner through a lower Scheldt dyke (Figure 7-1a). In contrast to the Halligen, vertical accretion is not desired in the polder to keep the water storage capacity of the polder at a constant level in order to maintain its protective function at storm surges. At mean tide conditions, a controlled flood of the polder takes place through an opening in the upper area of the Scheldt dyke (Figure 7-1b), because during moderate weather conditions the SPM concentration decreases with increasing water level. However, a complete avoidance of inflowing sediment cannot be prevented and so the sediment settles directly in the area behind the smaller dyke due to the slow flooding and the calm hydrodynamic conditions. A controlled drainage flow in the ebb phase (Figure 7-1c) through a single tidal gate generates a strong outgoing current which exports some recently deposited sediments out of the polder (Maris et al. 2007). Under these conditions, a long-term adaptation to the local MHW increase is significantly lower in the polder compared to the adjacent natural marsh (Vandenbruwaene et al. 2011). 
a)

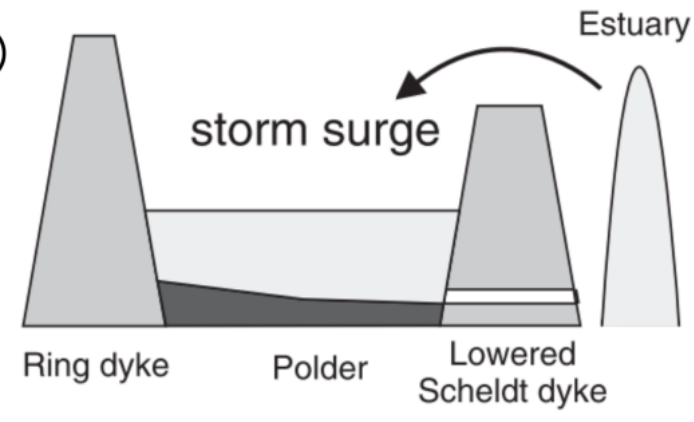

b)

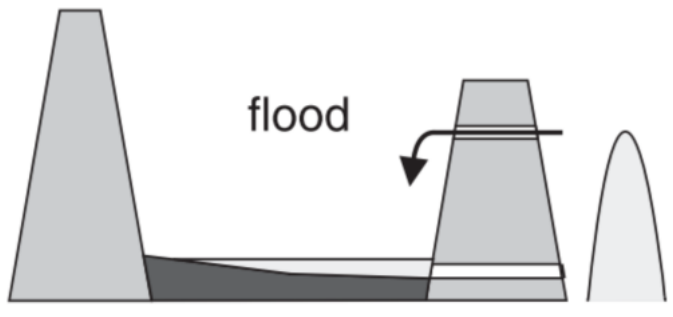

c)

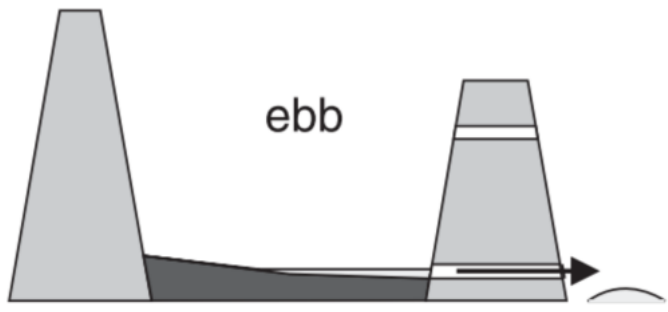

Figure 7-1: Modified Sketch of the working principle of the controlling flooded polder after Vandenbruwaene et al. (2011), illustrated for a storm surge (a), and for a mean tide during flood (b) and during ebb (c).

This example of a polder in the Scheldt estuary demonstrates that the main SPM transport mechanisms can be applied to the Halligen, as only a limited SPM transport towards the marshland takes place when dyke-like barriers such as revetments and breakwater constructions are overcome during inundations (Chapter 3, 4, 5). Furthermore, an undamped drainage water flow through tidal gates generates strong outgoing currents that most likely allow the remobilization of recently deposited sediments which are consequently washed away from the marshland surface (Chapter 4, 5). Remarkably, unlike the polder, an increased sediment transport towards the Halligen is necessary to enhance vertical accretion rates in order to keep pace with the local MHW rise. Hence, an adjustment of the coastal protection measures is therefore inevitable to mitigate the SPM transport mechanism issues in order to support their long-term preservation. 


\subsubsection{Tidal gate management}

Chapter 7.1 demonstrated that tidal gate management is one option to increase sediment accumulation rates as already suggested by Schindler (2014) and Hofstede et al. (2019b). In fact, it has been shown that the SPM loss in the drainage channel landside of a tidal gate is constantly higher than at the adjacent station on the marshland (Chapter 5). Furthermore, preventing SPM loss would have resulted in an additional vertical accretion rate of $27 \%$ in the measuring season $2019 / 20$. This high additional accretion rate clearly shows the necessity of slowing down the drainage water flow after storm surges. Slowing down the drainage water flow might be simply realized by partially opening tidal gates, for instance, with an obstacle attached on the seaside of a tidal gate. A more effective solution to slowing down the drainage water flow might be new tidal gates which are divided in several horizontal sections. These sections gradually open from top to bottom after certain time intervals and allow only the drainage of the upper water column which might contain less SPM because a certain proportion of sediments have already settled. Slowing down the drainage water flow by, for instance, just opening half of the tidal gates after storm surges is not advisable to avoid effects of flow channelling which may increase SPM loss (see Chapter 4).

Another possibility to increase sediment accumulation might be open tidal gates during inundations until slack water is reached to obtain a faster flooding of the marsh surface. An additional increase in sediment accumulation rates through open tidal gates during the flood phase is particularly relevant for Langeness as the orientation of five of the six tidal gates lie in the south which matches the main particle tracks across the southern Hallig margin towards the marshland (Chapter 4). Unfortunately, open tidal gate adjustments could not be tested within the project phase but will be discussed in Chapter 7.3.1.

\subsubsection{Barrier height adjustment}

Coastal protection measures like revetments and breakwater constructions (barriers) on Langeness have a SPM transport limiting effect that could be demonstrated by field measurements during moderate inundations (Chapter 3), storm surges (Chapter 5) and hydrodynamic model simulations (Chapter 4). Based on the model results, the most effective sediment accumulation increase is reached by adjusting the barrier heights along the whole southern barrier of Langeness (Case $C$ in Chapter 4). Barrier adjustments in the West and North of Langeness have also been examined. The results of these barrier adjustments showed less transported SPM and even an increased SPM loss. Particles that were released on northern 
tidal flats did not cross the northern margin of Langeness which might rather occur due to the particle transport directions prevailing there. These findings show that a general barrier adjustment solution for all Halligen cannot be implemented based on the model results of Langeness. Hence, for each Hallig, careful investigations of the local hydrodynamic conditions by means of hydrodynamic model-based simulations and accompanying sedimentological monitoring should first be conducted. Furthermore, in 1914 low dyke constructions named summer dyke were built on the two largest Halligen Hooge and Langeness (Müller and Fischer 1917) , which are supposed to impede inundations of the marsh areas during the summer months and reduce wave height during inundations (Mai et al. 1998). The latter property, however, decreases with increasing inundation height (Mai et al. 1998) and show no longer noteworthy effects at storm surges (Niemeyer and Kaiser 2001). These summer dykes almost surround the two Halligen and are located landside behind revetments and breakwater constructions (barriers) at a varying distance to the Hallig edge. Please note that the barriers were implemented to the model with higher resolution GNSS topographical survey data of the LKN.SH than the summer dyke on Langeness which was implemented into the model by a digital terrain model with a resolution of $1 \mathrm{~m}$. All height data sets, e.g. tidal flat bathymetric, marshland topographies and barrier heights, had to be interpolated to a single height file for the model calculations. The height resolution is lower on the summer dyke than at the barriers on the Hallig edge due to a coarser model grid to reduce computing time. Please note that the summer dyke is less pronounced at Hallig Langeness than at Hallig Hooge (Schindler et al. 2014b; Schindler and Willim 2015) and that a possible additional sediment transport-limiting effect cause by the summer dyke was not the purpose of the model. Hence, possible sediment transport limiting effects of the summer dyke are therefore not discussed according to the model results.

\subsection{Theoretical implementation of adjusted protection measures}

\subsubsection{Theoretical approach - same flooding frequency}

Schindler et al. (2014a) have already used two trap clusters on Hallig Langeness during a three year field campaign from October 2010 to March 2013, which generated representative accretion rates for the whole Hallig (Figure 7-2a). These trap clusters were used again at the same location, resulting in a six-year time series of vertical accretion rates. As already shown by 
the model results in Chapter 4 and by the storm surge measurements in Chapter 5 , the sediment accumulations and thus the annually averaged vertical accretion rates are most positively influenced by storm surges and their frequency (Figure 7-2b to $d$ ).
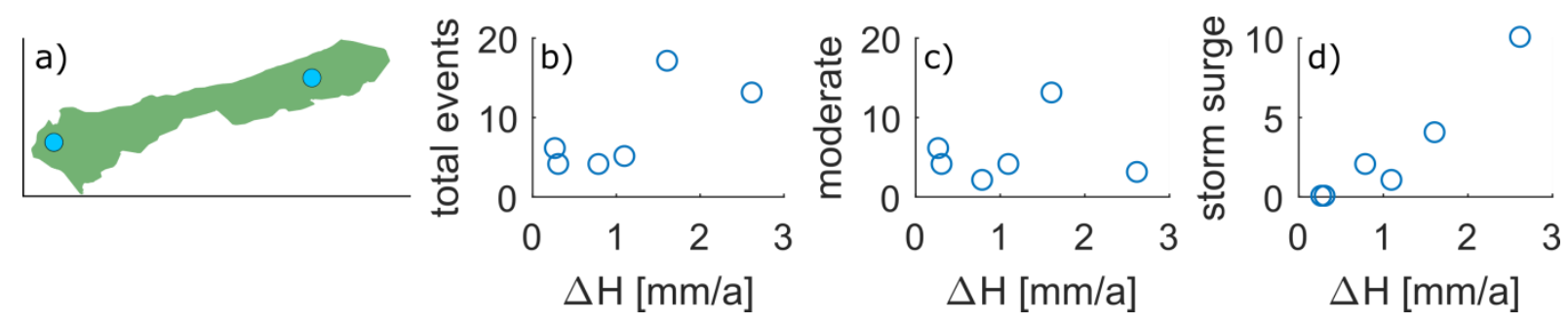

Figure 7-2: (a) shows the location of the two sediment trap cluster on Langeness (blue filled circles) where the sediment deposits were collected within six years to calculate annual vertical accretion rates. (b) shows the annual vertical accretion rates $(\Delta \mathrm{H})$ related to the total inundation events per year (c) $\Delta \mathrm{H}$ related to total moderate inundations per year and (d) $\Delta \mathrm{H}$ related to total storm surge induced inundations per year. The six-year time series includes annual vertical accretion data from 2010 to 2013 by Schindler et al. (2014a).

Based on the storm surge measurements (Chapter 5), storm surges generate on average about 4.2 times higher accretion rates per inundation than moderate events. Furthermore, the SPM loss after a storm surge is on average about the transported SPM that is needed to generate a vertical accretion rate of a single moderate inundation. Model results from Chapter 4 indicate that by adjusting the southern barrier height of Langeness, a doubling of the accretion rates would be possible during moderate inundations and an increase in the transported fine sand fraction of the SPM of up to $50 \%$ during storm surges. Based on the model (Chapter 4 ) and measurement results (Chapter 5), a theoretical scenario with adjusted protective measures and its increase in the mean accretion rates of the six-year time series (Figure 7-2) can now demonstrated. Hence, the adapted protection measures in the theoretical scenario include, (i) a decrease of the southern barrier height of Langeness such as the adjustment in Chapter 4 (Case C) and (ii) a slowing down of the drainage water flow through the tidal gates.

According to the theoretical scenario, preventing SPM loss generates additional accretion rates ranging between 0.14 and $0.58 \mathrm{~mm}$ (Table 7-1). An additional annual vertical accretion between 0.28 and $1.4 \mathrm{~mm}$ resulted only by adjusting the southern barrier heights. A combination of adjusting the barriers and preventing the SPM loss even generates an additional vertical accretion of up to $1.99 \mathrm{~mm}$ in 2019/20. The resulting total accretion rates of $3 \mathrm{~mm}$ in $2011 / 12$ and even up to $4.62 \mathrm{~mm}$ in $2019 / 20$ are able to significantly mitigate the imbalance to the local MHW rise of $5.0 \pm 0.3 \mathrm{~mm} / \mathrm{a}$ (1951-2011; Schindler et al. 2014a). These high annual 
vertical accretion rates, however, could only be gained due to higher storm surge frequencies in 2011/12 and 2019/20. A closer look at the six-year averaged accretion rate of $2.05 \mathrm{~mm} / \mathrm{a}$ demonstrates that the increased accretion rates are only sufficient to mitigate the imbalance to the local MHW rise, but not to compensate for it, despite the adjustments to the protection measures. On the basis of this theoretical approach, however, it can be argued that the accretion increase obtained by the barrier height adjustment might be approximately two times higher than the accretion increase through the pure prevention of SPM loss.

Table 7-1: Case A shows calculated annual vertical marshland accretion rates within six sampled years and as a six-year average ( 6 yr. av) of both sediment trap clusters (see Figure 7-2) as well as the total number of moderate inundations and storm surges and their six-year average. Same vertical accretion calculation but without SPM loss (A w. loss), or with adjusted southern barrier heights (C), or in combination by preventing SPM loss with adjusted southern barrier heights (C. w. loss).

\begin{tabular}{|c|c|c|c|c|c|c|c|}
\hline Cases & $2010 / 11$ & $2011 / 12$ & $2012 / 13$ & $2017 / 18$ & $2018 / 19$ & $2019 / 20$ & 6 yr. av. \\
\hline$A$ & 0.8 & 1.62 & 1.11 & 0.32 & 0.28 & 2.63 & 1.13 \\
\hline A w. loss & 0.95 & 1.84 & 1.25 & 0.32 & 0.28 & 3.21 & 1.31 \\
\hline C & 1.28 & 2.78 & 1.94 & 0.65 & 0.56 & 4.03 & 1.87 \\
\hline C w. loss & 1.43 & 3.00 & 2.07 & 0.65 & 0.56 & 4.62 & 2.05 \\
\hline $\begin{array}{l}\text { moderate } \\
\text { storm }\end{array}$ & 2 & 13 & 4 & 4 & 6 & 3 & 5.3 \\
\hline surge & 2 & 4 & 1 & 0 & 0 & 10 & 2.8 \\
\hline
\end{tabular}

A comparison with the annual accretion rates calculated from all trap clusters over the last three years shows that these accretion rates are significantly higher (Table 7-2) than the average accretion rates from the two trap clusters (Figure 7-2a, Table 7-1). This can be explained by the fact that in comparison to Schindler et al. (2014b), the positioning of the trap clusters were closer to the Hallig edge and the sediment accumulation decreases with distance to the sediment source (e.g. Christiansen et al. 2000; Temmerman et al. 2003b; Butzeck et al. 2015; Schindler et al. 2014a). The comparison of the annual accretion rates between Table 7-1 and Table 7-2 confirms that a levee build-up takes place under the current sediment transport conditions. It can be assumed that if the levee build-up continues to develop, an increase in a limiting effect on sediment transport conditions can be expected. 
Table 7-2: Case A shows calculated annual vertical marshland accretion rates within three sampled years and as a three year average ( $3 \mathrm{yr}$. av) of all 12 trap clusters. Same calculation but without SPM loss (Case $A$ w. loss), or with adjusted southern barrier heights (C), or in combination by preventing SPM loss with adjusted southern barrier heights (C. w. loss).

\begin{tabular}{ccccc}
\hline Cases & $\mathbf{2 0 1 7 / 1 8}$ & $\mathbf{2 0 1 8 / 1 9}$ & $\mathbf{2 0 1 9 / 2 0}$ & $\mathbf{3}$ yr. av. \\
\hline A & 0.9 & 1.0 & 7.4 & 3.1 \\
A w. loss & 0.9 & 1.0 & 9.04 & 3.64 \\
C & 1.8 & 2 & 11.34 & 5.04 \\
C w. loss & 1.8 & 2 & 12.99 & 5.59 \\
\hline
\end{tabular}

In general, the results of the theoretical scenario revealed that a representative accretion rate increase from $1.13 \mathrm{~mm} / \mathrm{a}$ to $2.05 \mathrm{~mm} / \mathrm{a}$ might be possible by the implementation of the adjusted coastal barriers and by preventing SPM loss at present inundation frequencies. This increase in vertical accretion rates, however, can only mitigate the imbalance to the local $\mathrm{MHW}$ rise of $5 \mathrm{~mm} / \mathrm{a}$. This assumption can be clarified by a comparison of a simple linear extrapolation between the vertical marshland accretion of Langeness from Schindler et al. (2014a) with $1.2 \mathrm{~mm} / \mathrm{a}$ and the increased vertical accretion rates of $2.05 \mathrm{~mm} / \mathrm{a}$. The result of this approximation revealed, if the mentioned adjustments would be implemented in 2020 , the MHW will reach the increased average ground level of Langeness (from 1.53 to $1.64 \mathrm{~mm}$ ) in 2063 instead of 2053. An ongoing levee build-up might even decrease the time slot of the 10 gained years as its construction leads to a progressive development of an additional SPM transport barrier especially for moderate events.

\subsubsection{Theoretical approach - increased flooding frequency}

In Chapter 3, measured sediment accumulations behind different barrier heights demonstrated that more sediment accumulate behind lower barriers. In Chapter 4 (case $C$ ), the higher barrier is set to the elevation of the lower barrier along the whole southern Hallig edge. A decrease of the southern barrier height may additionally increase the flooding frequency. To investigate this relationship, gauge level data of the outer tide gauge Hilligenley and the GNSS topographical survey dataset of the LKN.SH for the barrier heights are used.

Based on the inundation criterion in Chapter 3, an inundation of the entire Hallig occurs when the water level of both interior tide gauges reaches the threshold of the mean height of the non-permeable barriers heights with $2.34 \pm 0.19 \mathrm{~m}$ (Schindler and Willim 2015). An inundation, 
however, was not reached several times even though the outer tide gauge Hilligenley was above the threshold because of weak wind conditions or it was simply not enough time within a single tide to inundate the entire Hallig. These events are minor floods which can also occur at water heights at the outer tide gauge Hilligenley below the inundation threshold as the barriers vary in height around Langeness. The everyday life of the Hallig inhabitants, however, can even be impaired by the minor floods because they inundate at least parts of the marshland and are therefore considered. The minor floods especially affect the west of Langeness named Nordmarsch (Figure 7-3) due to its on average $0.13 \mathrm{~m}$ lower non-permeable barrier heights and its approximately $0.2 \mathrm{~m}$ lower terrain than the eastern part (Alt-Langeness) which is why Nordmarsch has generally more inundations per year then Alt-Langeness (Schindler and Willim 2015). For simplicity reasons, at least a minor flood occurs as soon as the outer tide gauge Hilligenley reached the thresholds according to the barrier height adjustments in Table 7-3.

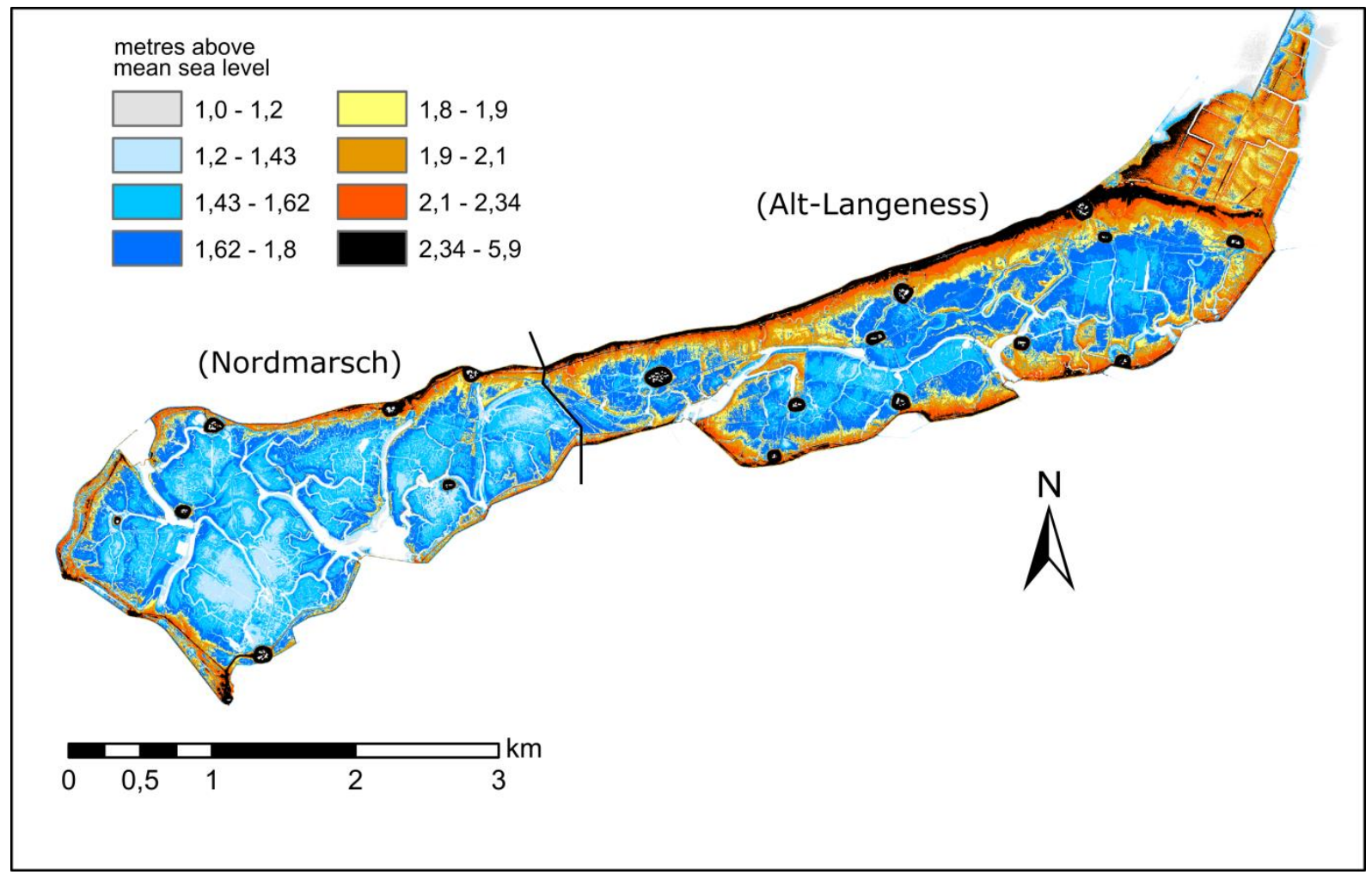

Figure 7-3: Digital ground model (based on Laser scan DGM1 data from 2014 courtesy of LKN.SH) showing depths from 1 to 5.9 metres above mean sea level. The $1.43 \mathrm{~m}$ threshold represents the mean ground level of Nordmarsch and $1.62 \mathrm{~m}$ of Alt-Langeness and $2.34 \mathrm{~m}$ represents the mean barrier height of Langeness according to Schindler and Willim (2015). The black line divides Langeness in its historical parts named Nordmarsch in the west and Alt-Langeness in the east. 
Table 7-3: Flooding frequency in total and its rounded annual average in brackets within the sampling period from October 2017 to March 2020. The additional floods are caused by the barrier height adjustment. Flooding beside season indicates the number of floods beside the annual storm season which last from October to March. Case $\mathrm{C}$ refers to the southern barrier height adjustment in Chapter 4.

\begin{tabular}{cccc}
\hline $\begin{array}{c}\text { barrier height } \\
\text { [m a.m.s.l.] }\end{array}$ & $\begin{array}{c}\text { flooding } \\
\text { in total }\end{array}$ & $\begin{array}{c}\text { additional } \\
\text { flooding }\end{array}$ & $\begin{array}{c}\text { flooding } \\
\text { beside season }\end{array}$ \\
\hline 2.34 & $35(12)$ & 0 & 0 \\
1.9 (Case C) & $158(53)$ & $123(41)$ & 16 \\
\hline
\end{tabular}

Based on the outer tide gauge level data Hilligenley, the mean barrier height of Langeness with $2.34 \mathrm{~m}$ was exceeded 35 times in total in the measurement period between October 2017 and March 2020 which also contains the minor floods (Table 7-3). Note that no flood occurred beside the winter storm seasons. The barrier adjustment to $1.9 \mathrm{~m}$ that represents case $\mathrm{C}$ in Chapter 4 increased the total flooding events by 123 minor floods up to 158 events in total by removing the breakwater constructions entirely. Note that this threshold reached 16 minor floods beside the winter storm seasons (Table 7-3).

The additional increase in the accretion rate due to the increased inundation frequency cannot be assessed as transported SPM measurements have not yet been carried out during minor floods. Nevertheless, it is well known that flooding frequency have a major impact on accretion rates (e.g. Stoddart et al. 1989; Temmerman et al. 2004; Schuerch et al. 2012). van Wijnen and Bakker (2001) even showed that accretion rates increase linearly with inundation frequency on natural marshes in the Wadden Sea. Hence, a 3.5-fold increase of today's flooding frequency (Table 7-3) might able to additionally increase the six-year averaged vertical accretion rate of $2.05 \mathrm{~mm} / \mathrm{a}$. 


\subsection{Further measures}

The theoretical implementation of adjusted protection measures highlighted the possibilities to increase vertical accretion rates through advantageous SPM transport conditions due to adjusted barrier heights and demonstrates a better utilization of the transported SPM by preventing SPM loss. In the following, further measures are discussed which support the previous adaptation measures in achieving increased sediment accumulation rates on the Halligen.

\subsubsection{Drainage channel connectivity}

The main principle of a drainage channel network on the Halligen is the fast drainage of the corresponding marshland after an inundation. In case of open tidal gates during the flood phase of an inundation, the drainage channels might turn into SPM transportation paths towards the marshland. The importance of channel systems for the transportation and distribution of sediments across the marsh surface was already demonstrated by Stoddart et al. (1989) on the Scolt Head Island, England. They measured high accretion values in the center of a marsh area which was locally influenced by a draining channel. Esselink et al. (1998) was also able to detect higher accretion rates within marshlands in the Ems Dollard estuary, Netherlands with wellmaintained artificial drainage systems than in marshes without these drainage systems. They assumed that the drainage systems may function as transportation paths for sediment to the marsh surface. Investigations by Schindler et al. (2014a) on Hallig Langeness were able to show high sediment deposition rates near the Hallig margin but not higher accretion rates next to a major drainage channel. In the following they reasoned that Langeness seems to be a transport limited landscape according to D'Alpaos et al. (2007) and that the obstructed and straightened channel system seems to prevent an effective sediment distribution to the Hallig center caused by tidal gates that close automatically at rising tides. First turbidity measurements with a single optical backscatter device (ASM-IV-L by Argus Gesellschaft für Umweltmeßtechnik mbH) within a drainage channel landside on Langeness were able to show that the sediment transport might be increased via open tidal gates during advantageous weather conditions (Schindler 2014). The latter author finally reasoned that the reactivation of the drainage channel systems on the Halligen might increase sediment accretion rates. In Chapter 5, it is demonstrated that the differences in sedimentation rates between Hallig edge and further towards the Hallig center decreases with a faster flooding of the Hallig surface. It is further assumed that open tidal gates 
during the flood phase of an inundation enhance a faster flooding of the marshland and thus supports a more homogeneous sediment accumulation pattern on the Hallig and in addition might counteract the ongoing process of a levee build-up (see Chapter 7.2.1). Finally, the reactivation of a broad drainage channel network might also be relevant for most occurring moderate inundations, as Temmerman et al. (2005a) was able to show that a high marsh platform was mainly supplied with sediment over the channel system and that the supply via the marsh edge primarily increases with increasing high water level. On the contrary, the drainage water flow should be slowing down during the ebb phase by e.g. partly opened tidal gates to mitigate the SPM loss (Chapter 5). Hence, we are also in favor of reactivating or even broadening the connectivity of today's straightened drainage channel system on the Halligen as SPM transportation network to enhance increased sediment accumulation rates and obtain a more effective sediment distribution pattern. An enhanced sediment trapping due to high plant stiffness (Peralta et al. 2008), should thus only be implemented with some distance to the drainage channels, as an early combing-out effect might support the levee build-up at the drainage channels edges. This assumption is also highlighted by the increased elevation along the drainage channels which is best captured in the east of Langeness (Figure 7-3).

\subsubsection{Land reclamation areas}

In Chapter 3 it was demonstrated that sediment in the fine sand range hardly reaches the marshland during moderate inundations. The adjustment to the coastal protection measure heights lead to almost a two-fold increase of sediment accumulation during moderate inundations but without any significant increase of transported fine sands (Chapter 4). An increase of the finer proportion of the SPM availability around the Halligen may increase the sediment accumulation at the most frequently occurring moderate inundations. This may be implemented by more land reclamation areas in proximity to the Hallig margins which enhance sediment settling of finer sediments without dissipating significant hydrodynamic forcing during high wind induced tides (Niemeyer and Kaiser 2001) comparable to moderate inundations. This measure could succeed as we were able to detect high SPM availabilities in the north of Langeness next to a land reclamation area (Chapter 2).

\subsubsection{Erosion interrelationships}

Conventional engineering solutions to mitigate or protect shorelines from hydrodynamic forcing are hard structures such as sea walls and embankments (e.g. Temmerman et al. 2013). Breakwater constructions, such as tetrapod which were implemented around the globe from 
India, France and even Germany on the island of Sylt (Daniel and Greslou 1962), are additional measures in order to dissipate hydrodynamic forces in advance at the foreshore. At least the larger Halligen are also encompassed with hard structures such as revetments, breakwater constructions and spur dykes (see Figure 3-2c, d). An ongoing trend to sustainable hydraulic engineering through "building with nature" achieved promising results, such as oysters and coral reefs in estuarine environments that provide habitat to a wide range of other species and have an additional coastal protection effect (Vriend et al. 2015). In general, however, regardless of if hard structures or an ecological solution is chosen, these measures always aim to dissipate hydrodynamic forces to decrease erosion processes. In Chapters 2 and 3, it could be demonstrated that energy dissipating measures such as spur dykes lead to predominantly low SPM availabilities. It could also be shown that the SPM availability under harsh weather conditions tends to be highest at locations with less protection against the open water (Chapter 2) and that SPM availability increases with increasing hydrodynamic forcing in the tidal flats (Chapter 5). Thus, the latter findings suggest that a significant proportion of the SPM availability is most likely generated locally on tidal flats in front of the marshland edge. Consequently, the more the coastal protection measure are based on dissipating hydrodynamic forcing on tidal flats, the more the SPM availability and thus the transported SPM will decrease.

It could be demonstrated that the barrier height adjustment in Chapter 4 led to a significant increase in sediment accumulation rates on the Hallig. A unique erosion protection strategy, however, on the marshland might be necessary after adjusting the barriers, managing the tidal gates and activating a broad drainage channel system as SPM transportation network. One solution to mitigate erosion could be adapted vegetation behind adjusted coastal barriers or on the drainage channel edges as submerged vegetation in salt marshes and mangrove forests in tidal areas can reduce flow velocity (Neumeier and Ciavola 2004; Temmerman et al. 2005b; Peralta et al. 2008) and have wave dissipation effects (e.g. Koch et al. 2009; Shepard et al. 2011) even at higher water levels and wave heights that are common at storm surges (Möller et al. 2014). In summary it can say that salt marshes in general can be a valuable component of coastal protection measures (Möller et al. 2014) but the magnitude of this value is not yet fully understood (Shepard et al. 2011). In general, the interactions between vegetation and physical erosion processes need to be tailored to realistic scales of action (Feagin et al. 2015). Hence, the implementation of adapted vegetation on the Halligen should be examined in the field as a matter of urgency in an ecological, sedimentological and engineering-related manner to prove 
the usefulness of erosion-reducing vegetation during various hydrodynamic scenarios ranging from moderate inundations to sever storm surges.

With an increased inflow of water masses through the drainage channels, other erosion protective measure solutions might become necessary. For instance, the drainage channels could be partly filled with rubble to decrease the shear stresses at the bottom. Drainage channel sections where high turbulences are to be expected (e.g. channel branching or narrow channels), might be stabilized with standardised rigid civil engineering structures such as concrete pipes or walls. However, pipes can become plugged with sediment after several inundations, which would make maintenance very time-consuming and expensive but would be necessary to maintain broad drainage channel connectivity. In general, it can be argued that the imbalance between vertical accretion rates and MHW rise is supported by today's coastal protection measures such as coastal barriers and tidal gates (Chapter 3, 4, 5). To our knowledge, in adjusting or even removing these measures, no reliable protection measures exist that could be used for protection against erosion and simultaneously allow a sufficiently high SPM transport towards the marshland that supports increased sediment accumulation rates. In view of the complex interrelationships between reaching an increased SPM transport without balancing the erosion potential, a unique erosion protection measure solution is needed for the Halligen.

\subsubsection{Impairments}

Nowadays, it can take up to two ebb phases until the water on the marshland is completely drained throughout the tidal gates after an inundation. With slower drainage water flow by managing the tidal gates, longer-lasting marshland inundation duration can be expected, depending on the tidal gate adjustment. In order not to impair the residents too much, a decreased drainage water flow might only necessary after storm surges, as no significant SPM loss were determined during moderate inundations (Chapter 4, 5). Based on the average number of occurred storm surges per year in the six-year period (Table 7-1), the inundation duration would increase by about 3 days per year if the drainage water flow will halve. Whether this reduction in the drainage water flow is sufficient to prevent SPM loss should first be verified by SPM measurements.

Height adjustments of the southern barriers (Chapter 4) and the increased SPM transport in the south of Langeness (Chapter 3, 4, 5) may speak in favour of corresponding adapted vegetation 
in the south of the Hallig in order to support the reduction of erosion or to increase sediment trapping. These areas are widely used as pasture grounds for livestock in the summer season, which makes a cross-season vegetation adaptation difficult as, for instance, canopy height decreases with increasing stocking rate (Andresen et al. 1990) and often leads to a less dense vegetation (Suchrow et al. 2012). The only solution would be to close certain areas to livestock in order to protect the vegetation which, however, would interfere with the lifestyle of the inhabitants.

In order to involve the needs of the Hallig inhabitants in the present research project and possible adaptation measures in the future, the inhabitants were interviewed. The results of the interviews by Krebs et al. (submitted) showed heterogeneous opinions of the Hallig inhabitants regarding the fundamental importance of the Hallig growth. Overall there is doubt about a sufficient growth of the Hallig through sediment deposits. According to the inhabitants, the increase in sedimentation rates should take place without excessive restrictions in everyday life. Adjustments or changes should therefore be made with reasonable sense and transparency with the involvement of the inhabitants. Hence, before an adjustment to the protection measures is implemented, the acceptance would have to be clarified with the Hallig inhabitants, as to whether they would be willing to accept an increasing impairment due to an increased flooding frequency, longer-lasting marshland inundation durations after storm surges and closed pasture grounds. In general, the interview results show greater need for public relations work, which increases the exchange between the research community and the population concerned, in order to clarify the usefulness of the measures examined and thus gain the acceptance of the inhabitants for future implementations of the measures. Therefore, it is important to take additional measures that could minimize the impairment of the residents. Hence, in view of the increased minor floods caused by adjusting the barrier height (Table 7-3), it is important to maintain the infrastructure for the inhabitants and tourism. This could be implemented with higher-lying traffic routes like those already on Hallig Hooge. Furthermore, the water masses from the minor floods during the winter season could be channelled with a sophisticated drainage channel network into areas that either have very low accretion rates or are not used for pasture grounds due to the high salt input. 


\section{Perspectives}

\subsection{Present state}

In general, one can say that the assessment of the present SPM availability on tidal flats around Hallig Langeness can also be adopted as a large-scale assessment to the tidal flats around the individual Halligen. This adoption is based on the fact that, for instance, Hallig Hooge has quite similar coastal protection measures and the recent average accretion rates by Schindler et al. (2014a) already showed lower average accretion rates on Langeness (1.2 $\pm 0.8 \mathrm{~mm} / \mathrm{a})$ compared to Hooge $(1.5 \pm 0.9 \mathrm{~mm} / \mathrm{a})$. Halligen with less coastal protective measures show significantly higher average accretion rates such as Nordstrandischmoor with $2.6 \pm 0.9 \mathrm{~mm} / \mathrm{a}$ (Schindler et al. 2014a) or the Hamburger Hallig with a mean surface elevation change of 6.18 $\mathrm{mm} / \mathrm{a}$ (Stock 2011). Nevertheless, when adjusting the coastal barrier heights at the individual Halligen, a SPM availability assessment on tidal flats around the individual Halligen may be beneficial to utilize the SPM availability at its best. In general, the SPM availability under harsh weather conditions tends to be highest at locations with less protection against the open water and lowest at shielded areas, for instance, between closely spaced spur dykes (Chapter 2). In addition, within the turbidity network the strongest wind dependency on high SPM availabilities was determined in the southeast of Langeness (Chapter 3), which is in line with a high increase of accumulated particles in the southeast of Langeness by adjusting the southern coastal protection measures (Chapter 4). However, smaller openings in the coastal protection measures lead to an increased SPM loss caused by higher flow velocities on the marshland at drainage flow conditions due to effects of flow channeling (Chapter 4) and should therefore be avoided entirely.

The outcome of the individual measure adjustment by contributing to a better SPM availability utilization may also depend on the different characteristic of the Hallig at which the adjustment is applied. In general, Chapters 3, 4 and 5 confirmed the general SPM transport-limiting effect of coastal barriers and the SPM loss due to unmanaged tidal gates that support rapid drainage water flow after inundations (Chapter 5). Nevertheless, the larger Halligen such as Hooge and Langeness must be additionally protected from the ongoing levee build-up (Chapter 7.2.1). These two Halligen are more affected by the levee build-up as higher barriers have an increased 
SPM transport limitation (Chapter 3) and forces settling processes in proximity to the Hallig edge. In addition, the larger surface area offers more space for decreasing sedimentation rates towards the Hallig center due to increasing distances to the sediment source. The latter two assumptions might be mitigated by ensuring a faster flooding of the Hallig through adjusting coastal barrier heights and through a broad drainage channel networking with open tidal gates during the flood phase of an inundation. Schindler et al. (2014a) calculated higher sediment transport rates and showed a more uniform distributed sediment accumulation pattern on the smaller Hallig Norstrandischmoor than on the larger Halligen Hooge and Langeness. They argued that in comparison to the larger Halligen the high sediment transport rates and the uniform sediment distribution pattern are most likely caused by the overall smaller distances to the sediment source and the lower coastal barrier heights. Chapters 3 to 5 confirmed that sediment accumulation increased with lower barrier heights and that transported SPM decreases with increasing distance to the sediment source. Thus, a high accretion increase is also likely by preventing SPM loss on smaller Halligen or at Halligen with lower coastal protection measures. The latter assumption is highlighted by the fact that with increasing transported SPM the SPM loss increases too (Chapter 5).

Adjusting the coastal protection measure such as height adjustments to the coastal barriers or slowing down the drainage water flow increased the six-year average vertical accretion from 1.13 to $2.05 \mathrm{~mm} / \mathrm{a}$ highlights the need for further measures that further mitigate or even compensate an MHW rise of $5 \mathrm{~mm} / \mathrm{a}$. Taking the increased flooding frequency into account might additionally increase the vertical accretion rates which, however, would result in distinct lifestyle impairments to the Hallig inhabitants due to an approximately 3.5-fold higher flooding frequency per year. One way to increase the tidal flat sediments for an increased SPM availability in the near future with less impairments of the Hallig inhabitants, land reclamation areas in proximity to the Hallig edges may be a solution. The finer grained SPM availability even reaches the marshland during the most frequently occurring moderate inundations (Chapter 3 ) which might increase sediment accumulation rates according to the theoretical estimation (Chapter 7.2.2). If more land reclamation areas were implemented, broadened drainage channel connectivity, tidal gate management and adapted vegetation cover become more relevant to utilize the finer grained SPM availability. With this already implemented, (i) the finer grain size distributed within transported SPM enables a more effective sediment distribution pattern even at lower hydrodynamic conditions, (ii) finer particles have low settling velocities and can remobilize more quickly at moderate hydrodynamic activity which makes preventing 
SPM loss due to slowing down of the drainage water flow and sediment trapping by vegetation more effective. In the case of Halligen with less agricultural activity, adapted vegetation is more likely to implement, due to the lack of livestock farming which makes it easier to adapt the vegetation across seasons and without interfering with the lifestyle of the Hallig inhabitants.

\subsection{Future state}

Storm surges have the potential to generate vertical accretion rates on the Halligen to keep pace with local MHW (Chapters 4, 5, 7). This is due to the fact that, with increasing hydrodynamic forcing, the SPM availability also increases as well as the transported SPM (Chapter 5). The probability of increasing storm surge frequencies (Befort et al. 2015) and higher storm surge water levels (Arns et al. 2015a) in the future might positively contribute to increased accretion rates on the Halligen. This scenario, however, is only valid if the presently assessed SPM availability does not decrease in the future. Hofstede and Stock (2018) predict that pending on SLR, sediment deficits in the Wadden Sea will increase. If that is the case, vertical accretion rates may decreases as a significant proportion of SPM availability is most likely generated locally on the tidal flats in front of the Hallig margin (Chapter 5). That SPM availability proportion might be lost in a future scenario were SLR increases the mean tidal range which result in constantly higher tidal forcing around the Halligen (Hofstede et al. 2019b). A constantly high tidal forcing during the absence of inundations is might able to decrease the proportions of finer bed sediments e.g. fine silt-sized material that is mostly present on top of the predominantly covered fine sand flats along Halligen shorelines after calm hydrodynamic periods (Chapter 3,5$)$. The effect of a lack of fine bed materials during an inundation can be demonstrated by the low SPM availability during a moderate inundation that occurred just five days after an earlier inundation without a distinct calming of the weather conditions in between (Chapter 3). Hence, a lack of fine grained sediments as additional source on tidal flats for the SPM availability may decrease the transported SPM during especially moderate inundations and thus a decrease of their caused vertical accretion rates on the Halligen might be expected. The hydro-mophodynamic simulations of two tidal basins (Lister Tief and Piep) in the southern North Sea under different SLR projections $(2.5,6,7$ and $17 \mathrm{~mm} / \mathrm{a})$ for two time horizons (2010 to 2050 and 2050 to 2100 ) always show an increase in the tidal range (Hofstede 
et al. 2019a). The latter authors conclude that even if mean SLR increases with $7 \mathrm{~mm} / \mathrm{a}$, the intertidal flats still function as effective sediment sinks. This assumption is based on using two particle classes $(60 \mu \mathrm{m}$ and $200 \mu \mathrm{m})$ in their sediment transport model. Consequently, at least the very fine to fine sand fraction would be still available in the future as source for a transported SPM towards the Halligen according to their modeled scenarios. In such a scenario, adjusting the barrier height would be a promising option to guarantee increased sediment accumulation rates in the future as the transported SPM within the fine sand range increased by $50 \%$ during the storm surges according to the model results of the $80 \mu \mathrm{m}$ particle tracks.

\subsection{Recommendation}

According to Hofstede and Stock (2018), Schleswig-Holstein State Government adopted an integrated climate change adaptation strategy in 2015 for the Schleswig-Holstein sector of the Wadden Sea in dealing with negative consequences for natural conservation and for coastal risk management. The main aim is the long-term maintenance of present functions and structures as well as the integrity of the Wadden Sea ecosystem in a changing climate. The first outcome of the strategy is that extra adaptation measures will not be necessary in the coming decades to address sediment deficits in the Wadden Sea due to climate change. For the Halligen in particular, the main focus on adaptation strategies should lay on measures that favour a sufficient number of inundations that transported sufficiently amounts of settable sediment towards the Halligen (Hofstede et al. 2019b). The latter authors further indicate to the importance of (i) a design change of the present coastal barriers, (ii) a certain management of the tidal gates (iii) and in managing the land use. Nevertheless, we would like to point out that adaption measures such as adjusting the barrier heights and preventing SPM loss almost doubled the six-year averaged vertical accretion rates to $2.05 \mathrm{~mm} / \mathrm{a}$ at current inundation frequencies. It is recommended to implement these measures in the near future where climate change might have no negatively foreseeable consequences on the presently assessed SPM availability and its utilization. Hence, an early implementation of these measures might mitigate the scenario by Hofstede et al. (2019b), in which the socio-economic uses of the Halligen would be restricted until 2050 due to an ongoing imbalance between vertical accretion rates and SLR. This scenario is highlighted by a comparison of a simple linear extrapolation between vertical accretion rates, with and without adjustments to the barrier heights and preventing SPM loss 
revealed that MHW reached the increased average ground level of Langeness ten years later in 2063. Taking an increased flooding frequency into account would most likely additional increase the vertical accretion rates and therefore the ten-year time slot. Additional time might be gained in which one can investigate and implement new strategies on maintaining resilience through the further discussed measures such as (i) open tidal gates during the flood phase of an inundation for an increased transported SPM, (ii) reactivating and broadening the drainage channel connectivity as an SPM transportation network to reach faster flooding and better sediment distribution on the marshland, (iii) more land reclamation areas in proximity to the Hallig margin to store additional SPM availability sources for inundations (iv) and an adapted vegetation cover that may increase sediment trapping and decrease erosion. For the implementation of the adjusted measures, we recommend maintaining a close exchange with the Hallig inhabitants with regards to the increased impairment of their everyday life because of the increased flooding frequency, increased inundation durations and closed pasture grounds. An increased acceptance of the inhabitants also promises to be able to put the theoretical measures faster into practice. We also want to point out that an increased erosion potential possibly caused by the discussed adaptation measures should also be in focus of following research projects.

The interdisciplinary collaboration carried out in the joint research project Living Coast Lab already showed effective means of opening up the examined adaptation strategies to Hallig Langeness for the entire Hallig world. These adaptation strategies might also be relevant for affected island- or mainland marshes where a SPM transport limitation takes place due to manmade coastal barriers. 


\section{Summary}

The resilience of coastal regions to SLR depends mainly on the availability of sediment in the intertidal areas and their redistribution in supratidal areas. Especially in highly dynamic tide dominated areas, this constantly repeating process is controlled by the suspension of bed sediments in the tidal flats and their tidal transport to the marshland. This repeating process ensures a vertical accretion of the marshes in the long term. Anthropogenic interventions such as dykes and breakwater constructions might disrupt this process. These anthropogenic interventions also apply to island-like marsh areas in the North Frisian Wadden Sea (southern North Sea), the so-called Halligen (Chapter 1). Previous studies were able to prove that today's vertical accretion rates are out of balance with SLR. This inevitably leads to the question of whether this imbalance is triggered by insufficient sediment availability for transportation towards the Hallig or whether the availability is sufficiently available but is not transported efficiently towards the marsh surface due to presently existing coastal protection measures. To answer this crucial question, we developed an autonomous working turbidity measurement system around and on the largest Hallig Langeness (Chapter 2, Hache et al. 2019). With a specially developed calibration method it was possible to convert a non-quantifiable unit (turbidity) into a physical quantity (grams per liter) in order to make any assessments about the suspended particulate matter (SPM). Furthermore, the tidal flat sediment around Langeness were investigated in a survey covering every $100 \mathrm{~m}$ within $500 \mathrm{~m}$ long transects perpendicular to the shoreline. The grain size analysis revealed a mixed sediment flat with predominantly fine sand along with a small increase of the grain size towards the tidal channels. Only a sheltered area between two spur dykes revealed a tidal flat with a finer grain size distribution with a higher proportion of silt-sized material. The first turbidity measurement season already revealed a high and systematic spatial and temporal SPM variability around Langeness between 0.04 and $0.56 \mathrm{~g} / \mathrm{l}$. SPM controlling factors include installation altitude, the proximity to tidal channels, the positive influence on SPM availability by adjacent land reclamation areas or the negative influence on SPM availability due to a shielded position between two spur dykes. The highest SPM concentrations were found in areas that are more exposed to the open sea. Further results show a strong increase in SPM availability under harsh weather conditions and inundation events but strong winds alone are not sufficient for a significant SPM increase. Instead, the combination of the appropriate southwestern wind direction and the strength and 
duration of the winds play a major role. The additional use of sediment accumulators at all turbidity measuring stations enabled a precise characterization of the suspended sediments and a combination of two different types of sediment traps allowed a closer look at accumulated sediments. This network of turbidity measuring stations, sediment accumulators and sediment trap fields generated a variety of data that gave us the opportunity for a detailed investigation of SPM concentrations on tidal flats (SPM availability) and SPM concentrations that reach the marshland surface (transported SPM) during eight moderate inundation events (Chapter 3, Hache et al. 2020). In order to be able to compare the individual inundations, water levels from surrounding tide gauges as well as wind data and wave data were evaluated. Herewith, we were able to indicate a strong dependency of the SPM availability around Langeness with wind conditions. Hence, constantly rising wind speeds over several tides from relatively stable southwestern directions are the conditions most likely to generate high SPM availabilities. A large spatial scale assessment of SPM availabilities and averaged sediment accumulation per inundation on the marshland showed a positive correlation. Furthermore, we were able to determine an SPM availability threshold of $0.15 \mathrm{~g} / \mathrm{l}$, which should at least be available on the tidal flats in order to allow sufficient vertical marshland accretion. However, this threshold is exceeded by the determined SPM availabilities but the transported SPM on the marshland after passing the coastal protection measures is at most $20 \%$. Grain size analysis of sediment accumulator samples on tidal flats and sediment trap samples on the marshland revealed that the deficit affects especially grain sizes between 63 and $200 \mu \mathrm{m}$ that can account for up to $70 \%$ of the sampled SPM on tidal flats. The measured sediment accumulation behind coastal protection measures of different heights at three different locations showed consistently higher sediment accumulations behind lower measures. With a detailed hydrodynamic numerical model of Langeness and the surrounding tidal flats, we made height adjustments to the coastal protection measures and investigated the effects on the SPM transport during moderate inundations and strong inundations (storm surges) using Lagrangian particle tracking with two particle classes 30 and $80 \mu \mathrm{m}$ (Chapter 4, Hache et al. 2021). The particle tracking results with adjusted coastal protection measures were compared with particle tracking results without making any changes to the model (status quo) to be able to determine the effect of coastal protection measure adjustments on differences in sediment accumulations. Hence, the height adjustments of the coastal protection measures resulted in a sediment accumulation increase up to $96 \%$ of the $30 \mu \mathrm{m}$ particles during moderate inundations but the $80 \mu \mathrm{m}$ particles remained unused. Status quo simulations under storm surge conditions 
showed a much higher potential for sediment accumulation in comparison to moderate inundations, reaching up to almost an eight-fold sediment accumulation increase by just consider the $30 \mu \mathrm{m}$ particles. Furthermore, storm surges are able to transport significant amounts of $80 \mu \mathrm{m}$ particles towards the marshland and an adjustment to coastal protection measures even increased this particle class up to $50 \%$. Apart from that, first insights into the SPM transport dynamics could be gained during storm surges that determined a SPM loss. The SPM loss is a sediment quantity that does not remain on the Hallig surface until the storm surge is over, i.e. the particles never settled or became remobilized again. Such SPM loss during storm surges is often higher than the total amount of accumulated particles during moderate inundation simulations. The model results indicate that moderate inundations are not able to generate any vertical accretion rates to compensate SLR despite the coastal protection measure adjustments. However, storm surges are able to generate the required accretion rates according to the model. This potential can only be fully utilized if the SPM loss is prevented by means of suitable protective measures. Finally, we succeeded in measuring and evaluating seven further inundations under storm surge conditions with our turbidity measurement network (Chapter 5, Hache et al. submitted). The comparison to moderate inundations revealed an up to 3.5-fold increase of the SPM availability and a higher grade of mixing within the water column due to increasing hydrodynamic forcing at storm surges. The high water levels and the strong hydrodynamic forcing lead to a decreased hampering effect of coastal protection measures which allows transported SPM within the fine sand fraction. A maximum of transported SPM of $73 \%$ of SPM availability thus is reached during the strongest of all measured storm surges with respect to wind speed, tidal range and flooding time. The storm surges generated vertical accretion rates that are capable to compensate SLR which are on average 4.2 times higher per inundation than at moderate events. SPM measurements in a drainage channel landside of a tidal gate during drainage flow conditions after a storm surge confirmed an existing SPM loss. A theoretical approach revealed that the average vertical accretion rates could significantly increase if SPM loss could be prevented by adjusted coastal management strategies. This SPM loss could be mitigated with adapted marshland vegetation as has been shown by an investigation of functional plant traits on a large data set of accretion rates within three different marsh types (island marsh, mainland marsh and Hallig marsh) in the German Wadden Sea region (Chapter 6, Bass et al. in prep.). The effects on accretion rates of functional plant traits and distance to the marsh edge were studied by using multiple regression analyses. In general, the mean accretion in all three investigated marsh types was 
below SLR. However, plants with a high above-ground roughness positively support accretion rates and plants with a stronger anchoring capacity can reduce negative impact on accretion rates such as erosion. For the Halligen, a general vegetation adaption is recommended which includes a lower plant height near the Hallig edge and a higher plant height towards the Hallig center to reach a better sediment accumulation distribution due to a comp-out effect. Based on the new findings of the Ph.D.-thesis, Chapter 7 highlights the main issue of the Halligen in gaining high vertical accretion rates despite the presented adaptation strategies. A comparison of a six-year data series of accretion rates within a theoretical approach, in which an SPM loss is prevented and adjusted coastal protection measures have been implemented on Langeness, has been carried out. Despite the adjustment measures on tidal gates and coastal protection measures, the six-year averaged accretion rate increases from 1.13 to $2.05 \mathrm{~mm} / \mathrm{a}$ with today's inundation frequency suggesting that the accretion rates are only sufficient to mitigate the imbalance to the local MHW rise, but not to compensate it. Taking the increased flooding frequency caused by lower barrier heights into account might additionally increase the vertical accretion rates due to an approximately 3.5-fold higher flooding frequency per year. Moreover, it can be assumed that the erosion potential increases by decreasing coastal protection measures heights and increased current velocities due to open tidal gates during the flood phase. Hence, future research projects should focus on investigating reliable protection that could be used for protection against erosion and simultaneously allows a sufficiently high SPM transport towards the Halligen. An increased impairment of the Hallig inhabitants through side effects of the adaption strategies such as increasing flooding frequencies, longer inundation times and impaired pasture management point to the need of involving the inhabitants in the implementation of the adaptation measures. In Chapter 8, the present assessment of the SPM availability around Langeness can be adopted as large-scale assessment to the tidal flats around the individual Halligen. The different characteristics of the individual Halligen suggest that especially larger Halligen must be protected with adjusted protection measures from the ongoing levee build up and that smaller Halligen or Halligen with lower coastal protection measures might gain higher vertical accretion by preventing SPM loss than larger Halligen. In addition, the adjustment of the coastal protection measure heights seems to be the best solution to increase vertical accretion rates in a future scenario where a deficit of fine tidal flat sediments is assumed. Finally, an implementation of the discussed coastal protection adjustments is recommended in the near future to mitigate a scenario where the socioeconomic use of the Halligen might be restricted until 2050. 


\section{References}

Agrawal, Y. C.; Whitmire, Amanda; Mikkelsen, Ole A.; Pottsmith, H. C. (2008): Light scattering by random shaped particles and consequences on measuring suspended sediments by laser diffraction. In $J$. Geophys. Res. 113 (C4), p. 1787. DOI: 10.1029/2007JC004403.

Ahrendt, Kai (2007): Vergangenheit und Zukunft des nordfriesischen Wattenmeeres. In Coastline Reports 9, pp. 45-57. Available online at http://epub.sub.uni-hamburg.de/epub/volltexte/2009/4085/.

Albers, Thorsten; Lieberman, Nicole von (2007): Morphologische Veränderungen von Wattflächen am Beispiel des Neufelder Sandes in der Elbmündung. In Coastline Reports 9, pp. 81-92. Available online at http://epub.sub.uni-hamburg.de/epub/volltexte/2009/4085/.

Allison, Mead A.; Yuill, Brendan T.; Meselhe, Ehab A.; Marsh, Jonathan K.; Kolker, Alexander S.; Ameen, Alexander D. (2017): Observational and numerical particle tracking to examine sediment dynamics in a Mississippi River delta diversion. In Estuarine, Coastal and Shelf Science 194, pp. 97-108. DOI: 10.1016/j.ecss.2017.06.004.

Andersen, T. J.; Pejrup, M. (2001): Suspended sediment transport on a temperate, microtidal mudflat, the Danish Wadden Sea. In Marine Geology 173 (1-4), pp. 69-85. DOI: 10.1016/S0025-3227(00)00164-X.

Andersen, T. J.; Svinth, S.; Pejrup, M. (2011): Temporal variation of accumulation rates on a natural salt marsh in the 20th century - The impact of sea level rise and increased inundation frequency. In Marine Geology 279 (1-4), pp. 178-187. DOI: 10.1016/j.margeo.2010.10.025.

Anderson, M. E.; Smith, J. M. (2014): Wave attenuation by flexible, idealized salt marsh vegetation. In Coastal Engineering 83, pp. 82-92. DOI: 10.1016/j.coastaleng.2013.10.004.

Andresen, H.; Bakker, J. P.; Brongers, M.; Heydemann, B.; Irmler, U. (1990): Long-term changes of salt marsh communities by cattle grazing. In Vegetatio 89 (2), pp. 137-148.

Arns, A.; Wahl, T.; Dangendorf, S.; Jensen, J. (2015a): The impact of sea level rise on storm surge water levels in the northern part of the German Bight. In Coastal Engineering 96, pp. 118-131. DOI: 10.1016/j.coastaleng.2014.12.002.

Arns, Arne; Dangendorf, Sönke; Jensen, Jürgen; Talke, Stefan; Bender, Jens; Pattiaratchi, Charitha (2017): Sea-level rise induced amplification of coastal protection design heights. In Scientific reports 7 , p. 40171. DOI: 10.1038/srep40171.

Arns, Arne; Wahl, Thomas; Haigh, Ivan D.; Jensen, Jürgen (2015b): Determining return water levels at ungauged coastal sites. A case study for northern Germany. In Ocean Dynamics 65 (4), pp. 539-554. DOI: 10.1007/s10236-015-0814-1.

Arns, Arne; Wahl, Thomas; Wolff, Claudia; Vafeidis, Athanasios T.; Haigh, Ivan D.; Woodworth, Philip et al. (2020): Non-linear interaction modulates global extreme sea levels, coastal flood exposure, and impacts. In Nature communications 11 (1), p. 1918. DOI: 10.1038/s41467-020-15752-5.

Artigas, Francisco; Shin, Jin Young; Hobble, Christine; Marti-Donati, Alejandro; Schäfer, Karina V.R.; Pechmann, Ildiko (2015): Long term carbon storage potential and CO 2 sink strength of a restored salt marsh in New Jersey. In Agricultural and Forest Meteorology 200, pp. 313-321. DOI:

10.1016/j.agrformet.2014.09.012.

Asmus, Ragnhild; Eskildsen, Kai; Garthe, Stefan; Reimers, Hans-Christian (2016): STopP-Abschlussbericht; Vom Sediment zum Top-Prädator. Available online at http://epic.awi.de/44179/1/STopP-

Abschlussbericht_online_version.pdf. 
Badewien, Thomas H.; Zimmer, Elke; Bartholomä, Alexander; Reuter, Rainer (2009): Towards continuous long-term measurements of suspended particulate matter (SPM) in turbid coastal waters. In Ocean Dynamics 59 (2), pp. 227-238. DOI: 10.1007/s10236-009-0183-8.

Baeye, Matthias; Fettweis, Michael; Voulgaris, George; van Lancker, Vera (2011): Sediment mobility in response to tidal and wind-driven flows along the Belgian inner shelf, southern North Sea. In Ocean Dynamics 61 (5), pp. 611-622. DOI: 10.1007/s10236-010-0370-7.

Balke, Thorsten; Stock, Martin; Jensen, Kai; Bouma, Tjeerd J.; Kleyer, Michael (2016): A global analysis of the seaward salt marsh extent. The importance of tidal range. In Water Resour. Res. 52 (5), pp. 37753786. DOI: 10.1002/2015WR018318.

Barbier, Edward B.; Hacker, Sally D.; Kennedy, Chris.; Koch, Evamaria W.; Stier, Adrian, C.; Silliman, Brian R. (2011): The value of estuarine and coastal ecosystem services. In Ecological Monographs 81 (2), pp. 169-193.

Bartholdy, J.; Aagaard, T. (2001): Storm surge effects on a back-barrier tidal flat of the Danish Wadden Sea. In Geo-Mar Lett 20 (3), pp. 133-141. DOI: 10.1007/s003670000048.

Bartholomä, Alexander; Kubicki, Adam; Badewien, Thomas H.; Flemming, Burghard W. (2009):

Suspended sediment transport in the German Wadden Sea-seasonal variations and extreme events. In Ocean Dynamics 59 (2), pp. 213-225. DOI: 10.1007/s10236-009-0193-6.

Bass, Julia; Granse, Dirk; Hache, Ingo; Jensen, Kai; Karius, Volker; Minden, Vanessa et al. (in prep.): Plant traits affect surface elevation change in salt marshes: an example of biogeomorphic ecosystems.

Becherer, Johannes; Flöser, Götz; Umlauf, Lars; Burchard, Hans (2016): Estuarine circulation versus tidal pumping. Sediment transport in a well-mixed tidal inlet. In J. Geophys. Res. Oceans 121 (8), pp. 62516270. DOI: 10.1002/2016JC011640.

Befort, D. J.; Fischer, M.; Leckebusch, G. C.; Ulbrich, U.; Ganske, A.; Rosenhagen, G.; Heinrich, H. (2015): Identification of storm surge events over the German Bight from atmospheric reanalysis and climate model data. In Nat. Hazards Earth Syst. Sci. 15 (6), pp. 1437-1447. DOI: 10.5194/nhess-15-1437-2015.

Benns, E. J.; Pilgrim, D. A. (1994): The effect of particle characteristics on the beam attenuation coefficient and output from an optical backscatter sensor. In Netherlands Journal of Aquatic Ecology 28 (3-4), pp. 245-248. DOI: 10.1007/BF02334191.

Black, K. P.; Rosenberg, M. A. (1994): Suspended sand measurements in a turbulent environment: field comparison of optical and pump sampling techniques. In Coastal Engineering 24, pp. 137-150. DOI: 10.1016/0378-3839(94)90030-2.

Blankespoor, Brian; Dasgupta, Susmita; Laplante, Benoit (2014): Sea-level rise and coastal wetlands. In Ambio 43 (8), pp. 996-1005. DOI: 10.1007/s13280-014-0500-4.

Bleninger, T.; Fenton, J. D.; Zentgraf, R. (2006): One-dimensional flow modelling and a case study of the River Rhine. Paper presented at the River Flow 2006. In Proc. Int. Conf. on Fluvial Hydraulics.

Boorman, L. A.; Garbutt, A.; Barratt, D. (1998): The role of vegetation in determining patterns of the accretion of salt marsh sediment. In Geological Society, London, Special Publications 139 (1), pp. 389399.

Borchert, Sinéad M.; Osland, Michael J.; Enwright, Nicholas M.; Griffith, Kereen T.; Rohr, Jason (2018): Coastal wetland adaptation to sea level rise. Quantifying potential for landward migration and coastal squeeze. In J Appl Ecol 55 (6), pp. 2876-2887. DOI: 10.1111/1365-2664.13169. 
Bouma, T.; Vries, M. de; Low, E.; Peralta, G.; Tánczos, I. v.; van de Koppel, J.; Herman, P. M. J. (2005): Trade-offs related to ecosystem engineering: A case study on stiffness of emerging macrophytes. In Ecology 86 (8), pp. 2187-2199.

Bouma, T. J.; Vries, M. B. de; Herman, P. M. J. (2010): Comparing ecosystem engineering efficiency of two plant species with contrasting growth strategies. In Ecology 91 (9), pp. 2696-2704.

Brown, S. L. (1998): Sedimentation on a Humber saltmarsh. In Geological Society 139 (1), pp. 69-83.

Brownlie, W. R. (1981): Prediction of flow depth and sediment discharge in open channels. Report No. KH-R-43A, 1981. Pasadena, California (USA): W. M. Keck Laboratory of Hydraulics and Water Resources.

Bunt, Jamie A.C.; Larcombe, Piers; Jago, Colin F. (1999): Quantifying the response of optical backscatter devices and transmissometers to variations in suspended particulate matter. In Continental Shelf Research 19 (9), pp. 1199-1220. DOI: 10.1016/S0278-4343(99)00018-7.

Butzeck, C.; Eschenbach, A.; Gröngröft, A.; Hansen, K.; Nolte, S.; Jensen, K. (2015): Sediment Deposition and Accretion Rates in Tidal Marshes Are Highly Variable Along Estuarine Salinity and Flooding Gradients. In Estuaries and Coasts 38 (2), pp. 434-450. DOI: 10.1007/s12237-014-9848-8.

Cadol, Daniel; Engelhardt, Katharina; Elmore, Andrew; Sanders, Geoffrey (2014): Elevation-dependent surface elevation gain in a tidal freshwater marsh and implications for marsh persistence. In Limnol. Oceanogr. 59 (3), pp. 1065-1080. DOI: 10.4319/lo.2014.59.3.1065.

Campbell Scientific, Inc. (2017): OBS-3+ and OBS300 Suspended Solids and Turbidity Monitors. Instruction Manual campbell@ scientific. Revision: 2/17.

Chmura, Gail L.; Coffey, Amanda; Crago, Ryan (2001): Variation in Surface Sediment Deposition on Salt Marshes in the Bay of Fundy. In Journal of Coastal Research 17, pp. 221-227.

Chow, V. T. (1959): Open-channel hydraulics. New York: McGraw-Hill New York.

Christiansen, T.; Wiberg, P. L.; Milligan, T. G. (2000): Flow and Sediment Transport on a Tidal Salt Marsh Surface. In Estuarine, Coastal and Shelf Science 50 (3), pp. 315-331. DOI: 10.1006/ecss.2000.0548.

Conner, C. S.; Visser, A. M. de (1992): A laboratory investigation of particle size effects on an optical backscatterance sensor. In Marine Geology 108 (2), pp. 151-159. DOI: 10.1016/0025-3227(92)90169-I.

Copernicus Climate Change Service (C3S) (2017): ERA5: Fifth generation of ECMWF atmospheric reanalyses of the global climate. Copernicus Climate Change Service Climate Data Store (CDS), date of access. https://cds.climate.copernicus.eu/cdsapp\#!/home. DOI: 10.21957/vf291hehd7.

Corenblit, Dov; Baas, Andreas; Balke, Thorsten; Bouma, Tjeerd; Fromard, François; Garófano-Gómez, Virginia et al. (2015): Engineer pioneer plants respond to and affect geomorphic constraints similarly along water-terrestrial interfaces world-wide. In Global Ecology and Biogeography 24 (12), pp. 13631376. DOI: $10.1111 /$ geb.12373.

Costanza, R.; d'Arge, R.; Groot, R. de (1997): The value of the world's ecosystem services and natural capital. In Nature (387), pp. 253-260. DOI: 10.1038/387253a0.

Cox, Tom; Maris, Tom; Vleeschauwer, Pieter de; Mulder, Tom de; Soetaert, Karline; Meire, Patrick (2006): Flood control areas as an opportunity to restore estuarine habitat. In Ecological Engineering 28 (1), pp. 55-63. DOI: 10.1016/j.ecoleng.2006.04.001.

CPSL (2010): Third Report. The role of spatial planning and sediment coastal risk management. Wadden Sea Ecosystem No. 28. Common Wadden Sea Secretariat, Trilaterial Working Group on Coastal Protection and Sea Level Rise (CPSL). Wilhelmshaven, Germany. 
Craft, Christopher; Clough, Jonathan; Ehman, Jeff; Joye, Samantha; Park, Richard; Pennings, Steve et al. (2009): Forecasting the effects of accelerated sea-level rise on tidal marsh ecosystem services. In Frontiers in Ecology and the Environment 7 (2), pp. 73-78. DOI: 10.1890/070219.

D'Alpaos, A.; Mudd, S. M.; Carniello, L. (2011): Dynamic response of marshes to perturbations in suspended sediment concentrations and rates of relative sea level rise. In J. Geophys. Res. 116, F04020. DOI: 10.1029/2011JF002093.

D'Alpaos, Andrea; Lanzoni, Stefano; Marani, Marco; Rinaldo, Andrea (2007): Landscape evolution in tidal embayments. Modeling the interplay of erosion, sedimentation, and vegetation dynamics. In J. Geophys. Res. 112 (F1), p. 77. DOI: 10.1029/2006JF000537.

Daniel, P.; Greslou, L. (1962): THE TETRAPOD. In Coastal Engineering Proceedings 8, pp. 469-481.

Danielsen, Finn; Sørensen, Mikael K.; Olwig, Mette F.; Selvam, Vaithilingam; Parish, Faizal; Burgess, Neil D. et al. (2005): The Asian tsunami. A protective role for coastal vegetation. In Science (New York, N.Y.) 310 (5748), p. 643. DOI: 10.1126/science.1118387.

Davidson, Kate E.; Fowler, Mike S.; Skov, Martin W.; Doerr, Stefan H.; Beaumont, Nicola; Griffin, John N.; Bennett, Joseph (2017): Livestock grazing alters multiple ecosystem properties and services in salt marshes. A meta-analysis. In J Appl Ecol 54 (5), pp. 1395-1405. DOI: 10.1111/1365-2664.12892.

Davidson-Arnott RGD.; Van Proosdij D.; Ollerhead J.; Schostak L. (2002): Hydrodynamics and sedimentation in salt marshes: examples from amacrotidal marsh, Bay of Fundy. In Geomorphology 48, pp. 209-231. DOI: 10.1016/S0169-555X(02)00182-4.

Deicke, Matthias; Karius, Volker; Wiebke, Jahnke; Wiebke, Kallweit; Marlene, Rebens; Dorothea, Reyer (2007): Charakterisierung von Sturmflutablagerungen auf Hallig Hoog - Quantifizierung des Sedimentwachstums seit 1914. In Coastline Reports 9, 2007, pp. 93-102.

DHI (2017a): MIKE 21 \& MIKE 3 Flow Model FM. Particle Tracking Module - Scientific Documentation. Hg. v. Danish Hydraulic Institute (DHI).

DHI (2017b): MIKE 21 Flow Model FM. Hydrodynamic Module. User Guide. Hg. v. Danish Hydraulic Institute (DHI).

Donnelly, J. P.; Bertness, M. D. (2001): Rapid shoreward encroachment of salt marsh cordgrass in response to accelerated sea-level rise. In Proceedings of the National Academy of Sciences 98 (25), pp. 14218-14223.

Down, R. D.; Lehr, J. H. (2005): Environmental Instrumentation and Analysis Handbook. Chapter 24. Turbidity Monitoring, pp. 511-547. Hoboken, New Jersey: John Wiley \& Sons, Inc.

Downing, John P. (2006): Twenty-five years with OBS sensors. The good, the bad, and the ugly. In Continental Shelf Research 26, 2006 (17-18), pp. 2299-2318. DOI: 10.1016/j.csr.2006.07.018.

Downing, John P.; Beach, Reginald A. (1989): Laboratory apparatus for calibrating optical suspended solids sensors. In Marine Geology 86 (2-3), pp. 243-249. DOI: 10.1016/0025-3227(89)90053-4.

Druine, Flavie; Verney, Romaric; Deloffre, Julien; Lemoine, Jean-Philippe; Chapalain, Marion; Landemaine, Valentin; Lafite, Robert (2018): In situ high frequency long term measurements of suspended sediment concentration in turbid estuarine system (Seine Estuary, France). Optical turbidity sensors response to suspended sediment characteristics. In Marine Geology 400, pp. 24-37. DOI: 10.1016/j.margeo.2018.03.003. 
Elschot, Kelly; Bouma, Tjeerd J.; Temmerman, Stijn; Bakker, Jan P. (2013): Effects of long-term grazing on sediment deposition and salt-marsh accretion rates. In Estuarine, Coastal and Shelf Science 133, pp. 109-115. DOI: 10.1016/j.ecss.2013.08.021.

Esselink, P.; Dijkema, K. S.; Reents, S.; Hageman, G. (1998): Vertical accretion and profile changes in abandoned man-made tidal marshes in the Dollard estuary, the Netherlands. In Journal of Coastal Research 14 (2), pp. 570-582.

Feagin, Rusty A.; Figlus, Jens; Zinnert, Julie C.; Sigren, Jake; Martínez, Marisa L.; Silva, Rodolfo et al. (2015): Going with the flow or against the grain? The promise of vegetation for protecting beaches, dunes, and barrier islands from erosion. In Frontiers in Ecology and the Environment 13 (4), pp. 203-210. DOI: $10.1890 / 140218$.

Fettweis, Michael; Francken, Frederic; Pison, Virginie; van den Eynde, Dries (2006): Suspended particulate matter dynamics and aggregate sizes in a high turbidity area. In Marine Geology 235 (1-4), pp. 63-74. DOI: 10.1016/j.margeo.2006.10.005.

Fettweis, Michael; Francken, Frederic; van den Eynde, Dries; Verwaest, Toon; Janssens, Job; van Lancker, Vera (2010): Storm influence on SPM concentrations in a coastal turbidity maximum area with high anthropogenic impact (southern North Sea). In Continental Shelf Research 30 (13), pp. 1417-1427. DOI: 10.1016/j.csr.2010.05.001.

Fettweis, Michael; Monbaliu, Jaak; Baeye, Matthias; Nechad, Bouchra; van den Eynde, Dries (2012): Weather and climate induced spatial variability of surface suspended particulate matter concentration in the North Sea and the English Channel. In Methods in Oceanography 3-4, pp. 25-39. DOI: 10.1016/j.mio.2012.11.001.

Fettweis, Michael; Nechad, Bouchra; van den Eynde, Dries (2007): An estimate of the suspended particulate matter (SPM) transport in the southern North Sea using SeaWiFS images, in situ measurements and numerical model results. In Continental Shelf Research 27 (10-11), pp. 1568-1583. DOI: 10.1016/j.csr.2007.01.017.

Fettweis, Michael; Riethmüller, Rolf; Verney, Romaric; Becker, Marius; Backers, Joan; Baeye, Matthias et al. (2019): Uncertainties associated with in situ high-frequency long-term observations of suspended particulate matter concentration using optical and acoustic sensors. In Progress in Oceanography 178, p. 102162. DOI: 10.1016/j.pocean.2019.102162.

Folk, Robert Louis; Ward, C. William (1957): Brazos River bar [Texas]; a study in the significance of grain size parameters. In journal of Sedimentary Research 21, pp. 3-21. DOI: 10.1306/74D70646-2B21-11D78648000102C1865D.

Francalanci, S.; Bendoni, M.; Rinaldi, M.; Solari, L. (2013): Ecomorphodynamic evolution of salt marshes. Experimental observations of bank retreat processes. In Geomorphology 195, pp. 53-65. DOI: 10.1016/j.geomorph.2013.04.026.

French, Jon (2006): Tidal marsh sedimentation and resilience to environmental change. Exploratory modelling of tidal, sea-level and sediment supply forcing in predominantly allochthonous systems. In Marine Geology 235 (1-4), pp. 119-136. DOI: 10.1016/j.margeo.2006.10.009.

Fugate, David C.; Friedrichs, Carl T. (2002): Determining concentration and fall velocity of estuarine particle populations using ADV, OBS and LISST. In Continental Shelf Research 22 (11-13), pp. 1867-1886. DOI: 10.1016/S0278-4343(02)00043-2. 
Gardner, Wilford D. (1980a): Field assessment of sediment traps. In Journal of Marine Research 38, pp. 41-52.

Gardner, Wilford D. (1980b): Sediment trap dynamics and calibration: a laboratory evaluation. In Journal of Marine Research 38, pp. 17-39.

Garnier, Eric; Lavorel, Sandra; Ansquer, Pauline; Castro, Helena; Cruz, Pablo; Dolezal, Jiri et al. (2007): Assessing the effects of land-use change on plant traits, communities and ecosystem functioning in grasslands. A standardized methodology and lessons from an application to 11 European sites. In Annals of botany 99 (5), pp. 967-985. DOI: 10.1093/aob/mcl215.

Gaslikova, Lidia; Grabemann, Iris; Groll, Nikolaus (2013): Changes in North Sea storm surge conditions for four transient future climate realizations. In Nat Hazards 66 (3), pp. 1501-1518. DOI: 10.1007/s11069-012-0279-1.

Gätje, Ch; Reise, K. (1998): The Wadden Sea Ecosystem. Exchange, Transport and Transformation Processes. Berlin, Heidelberg, New York, Barcelona, Budapest, Hongkong, London, Mailand, Paris, Santa Clara, Singapur, Tokio: Springer Berlin Heidelberg.

Hache, Ingo; Karius, Volker; Eynatten, Hilmar von (submitted): Storm surge induced sediment accumulation on anthropogenic marshland in the Southern North Sea: Implications for coastal protection. In Estuarine, Coastal and Shelf Science.

Hache, Ingo; Karius, Volker; Eynatten, Hilmar von (2020): Suspended particulate matter for sediment accumulation on inundated anthropogenic marshland in the southern North Sea - Potential, thresholds and limitations. In Continental Shelf Research 207, pp. 1-14. DOI: 10.1016/j.csr.2020.104214.

Hache, Ingo; Karius, Volker; Gutkuhn, Jörg; Eynatten, Hilmar von (2019): The development and application of an autonomous working turbidity measurement network: Assessing the spatial and temporal distribution of suspended particulate matter on tidal flats in the North Frisian Wadden Sea. In Continental Shelf Research 176, pp. 36-50. DOI: 10.1016/j.csr.2019.02.010.

Hache, Ingo; Niehüser, Sebastian; Karius, Volker; Arns, Arne; Eynatten, Hilmar von (2021): Assessing sediment accumulation at inundated anthropogenic marshland in the southeastern North Sea: using particle tracking on modified coastal protection structures. In Ocean and Coastal Management 208. DOI: 10.1016/j.ocecoaman.2021.105631.

Hayes, M. O. (1979): Barrier island morphology as a function of tidal and wave regime. In: Leatherman, S.P. (Ed.), Barrier Islands from the Gulf of St. Lawrence to the Gulf of Mexico. In Academic Press, New York, 1979, pp. 211-236.

Heyer, Holger; Schrottke, Kerstin (2013): Aufbau von integrierten Modellsystemen zur Analyse der langfristigen Morphodynamik in der Deutschen Bucht. AufMod; gemeinsamer Abschlussbericht für das Gesamtprojekt mit Beiträgen aus allen 7 Teilprojekten. Unter Mitarbeit von TIB - Technische Informationsbibliothek Universitätsbibliothek Hannover und Technische Informationsbibliothek (TIB).

Hofstede, Jacobus L. A. (1997): Process-Response Analysis for the North Frisian Supratidal Sands (Germany). In Journal of Coastal Research 13 (1), pp. 1-7.

Hofstede, Jacobus L. A. (1999): Regional differences in the morphologic behaviour of four German Wadden Sea barriers. In Quaternary International 56, pp. 99-106.

Hofstede, Jacobus L. A. (2015): Theoretical considerations on how Wadden Sea tidal basins may react to accelerated sea level rise. In Zeit für Geo 59 (3), pp. 377-391. DOI: 10.1127/zfg/2014/0163. 
Hofstede, Jacobus L. A. (2019): Küstenschutz in Schleswig-Holstein: ein Überblick über Strategien und Maßnahmen. In Die Küste 87. DOI: 10.18171/1.087103.

Hofstede, Jacobus L. A.; Becherer, Johannes; Burchard, Hans (2019a): Morphologische Projektionen für zwei Tidesysteme im Wattenmeer von Schleswig-Holstein: SH-TREND. In Die Küste 87. DOI: 10.18171/1.087101.

Hofstede, Jacobus L. A.; Matelski, Birgit; Stock, Martin (2019b): Schleswig-Holsteins KlimaAnpassungsstrategie für das Wattenmeer 2100. In Die Küste 87. DOI: 10.18171/1.087102.

Hofstede, Jacobus L. A.; Stock, Martin (2018): Climate change adaptation in the Schleswig-Holstein sector of the Wadden Sea. An integrated state governmental strategy. In J Coast Conserv 22 (1), pp. 199-207. DOI: 10.1007/s11852-016-0433-0.

Horton, Benjamin P.; Shennan, lan; Bradley, Sarah L.; Cahill, Niamh; Kirwan, Matthew; Kopp, Robert E.; Shaw, Timothy A. (2018): Predicting marsh vulnerability to sea-level rise using Holocene relative sealevel data. In Nature communications 9 (1), p. 2687. DOI: 10.1038/s41467-018-05080-0.

Hunter, John (2009): Estimating sea-level extremes under conditions of uncertain sea-level rise. In Climatic Change 99 (3-4), pp. 331-350. DOI: 10.1007/s10584-009-9671-6.

IPCC (Ed.) ((in press)): Summary for Policymakers. With assistance of In H. O. Pörtner, D. C. Roberts, V. Masson-Delmotte, P. Zhai, M. Tignor, E. Poloczanska, K. Mintenbeck, M. Alegria, M. Nicolai, A. Okem, J. Petzold \& B. W. Rama, N. M. (Eds.). Geneva, Switzerland.

Jäger, E. J. (2016): Rothmaler-Exkursionsflora von Deutschland. Gefäßpflanzen: Grundband. Heidelberg, Germany: Springer Spektrum.

Jensen, J.; Frank, T.; Wahl, T.; Dangendorf, S. (2011): Analyse von hochaufgelösten Tidenwasserständen und Ermittlung der MSL an der deutschen Nordseeküste (AMSeL). In Die Küste 78, pp. 59-163.

Jorge, V. N. de; van Beusekom, J. E. E. (1995): Wind- and tide-induced resuspension of sediment and microphytobenthos from tidal flats in the Ems estuary. In Limnol. Oceanogr. 40 (4), pp. 776-778. DOI: 10.4319/lo.1995.40.4.0776.

Kathiresan, Kandasamy; Rajendran, Narayanasamy (2005): Coastal mangrove forests mitigated tsunami. In Estuarine, Coastal and Shelf Science 65 (3), pp. 601-606. DOI: 10.1016/j.ecss.2005.06.022.

Kineke, G.C; Sternberg, R.W (1992): Measurements of high concentration suspended sediments using the optical backscatterance sensor. In Marine Geology 108, 1992 (3-4), pp. 253-258. DOI: 10.1016/00253227(92)90199-R.

Kirwan, Matthew L.; Guntenspergen, Glenn R.; D'Alpaos, Andrea; Morris, James T.; Mudd, Simon M.; Temmerman, Stijn (2010): Limits on the adaptability of coastal marshes to rising sea level. In Geophys. Res. Lett. 37, L23401. DOI: 10.1029/2010GL045489.

Kirwan, Matthew L.; Megonigal, J. Patrick (2013): Tidal wetland stability in the face of human impacts and sea-level rise. In Nature 504 (7478), pp. 53-60. DOI: 10.1038/nature12856.

Kirwan, Matthew L.; Murray, A. Brad (2008): Ecological and morphological response of brackish tidal marshland to the next century of sea level rise. Westham Island, British Columbia. In Global and Planetary Change 60 (3-4), pp. 471-486. DOI: 10.1016/j.gloplacha.2007.05.005.

Kirwan, Matthew L.; Temmerman, Stijn; Skeehan, Emily E.; Guntenspergen, Glenn R.; Fagherazzi, Sergio (2016a): Overestimation of marsh vulnerability to sea level rise. In Nature Clim Change 6 (3), pp. 253260. DOI: $10.1038 /$ nclimate2909. 
Kirwan, Matthew L.; Walters, David C.; Reay, William G.; Carr, Joel A. (2016b): Sea level driven marsh expansion in a coupled model of marsh erosion and migration. In Geophys. Res. Lett. 43 (9), pp. $4366-$ 4373. DOI: $10.1002 / 2016$ GL068507.

Kleyer, M. (1999): Distribution of plant functional types along gradients of disturbance intensity and resource supply in an agricultural landscape. In Journal of Vegetation science 10 (5), pp. 697-708.

Kleyer, M.; Balke, T.; Minden, V.; Peppler-Lisbach, C.; Schoenmakers, S.; Spalke, J.; Timmermann, H. (2014): Mellum: a highly dynamic landscape, though not for plants. In U. Hellwig \& M. Stock (Eds.). In Dynamic isalnds in the Wadden Sea, Wadden Sea Ecosystem Secretariat 33, pp. 29-43.

Kleyer, Michael; Trinogga, Juliane; Cebrián-Piqueras, Miguel A.; Trenkamp, Anastasia; Fløjgaard, Camilla; Ejrnaes, Rasmus et al. (2019): Trait correlation network analysis identifies biomass allocation traits and stem specific length as hub traits in herbaceous perennial plants. In J Ecol 107 (2), pp. 829-842. DOI: 10.1111/1365-2745.13066.

Knevel, I. C.; Bekker, R. M.; Kunzmann, D.; Stadler, M.; Thompson, K. (2005): The LEDA traitbase collecting and measuring standards of life-history traits of the Northwest European Flora. Groningen, Netherlands: University of Groningen, Community and Conservation Ecology Group.

Koch, Evamaria W.; Barbier, Edward B.; Silliman, Brian R.; Reed, Denise J.; Perillo, Gerardo M. E.; Hacker, Sally D. et al. (2009): Non-linearity in ecosystem services. Temporal and spatial variability in coastal protection. In Frontiers in Ecology and the Environment 7 (1), pp. 29-37. DOI: 10.1890/080126.

Kolker, Alexander S.; Kirwan, Matthew L.; Goodbred, Steven L.; Cochran, J. Kirk (2010): Global climate changes recorded in coastal wetland sediments. Empirical observations linked to theoretical predictions. In Geophys. Res. Lett. 37 (14), n/a-n/a. DOI: 10.1029/2010GL043874.

Krebs, Verena; Ziesen, Nenja; Wöffler, Teide; Häußling, Roger; Schüttrumpf, Holger (submitted): Teilstationäre Hochwasserschutzmaßnahmen als kurzfristige Strategie zum Schutz der nordfriesischen Halligen - Einsatztests unter praxisnahen Bedingungen und Berücksichtigung sozialer Bedürfnisse der Bewohner*innen. In Die Küste.

Langley, J. A.; McKee, K. L.; Cahoon, D. R.; Cherry, J. A.; Megonigal, J. P. (2009): Elevated CO2 stimulates marsh elevation gain, counterbalancing sea-level rise. In Proceedings of the National Academy of Sciences 106 (15), pp. 6182-6186.

Lavorel, S.; McIntyre, S.; Landsberg, J.; Forbes, T. D. A. (1997): Plant functional classifications: from general groups to specific groups based on response to disturbance. In Trends in Ecology \& Evolution 12 (12), pp. 474-478.

Lefebvre, A.; Thompson, C.E.L.; Amos, C. L. (2010): Influence of Zostera marina canopies on unidirectional flow, hydraulic roughness and sediment movement. In Continental Shelf Research 30 (16), pp. 1783-1794. DOI: 10.1016/j.csr.2010.08.006.

Liubartseva, S.; Coppini, G.; Lecci, R.; Clementi, E. (2018): Tracking plastics in the Mediterranean: 2D Lagrangian model. In Marine Pollution Bulletin 129 (1), pp. 151-162. DOI:

10.1016/j.marpolbul.2018.02.019.

Mai, S.; Daemrich, K. F.; Zimmermann, C. (1998): Wellentransmission an Sommerdeichen. In Wasser und Boden 11, pp. 28-40.

Manning, R. (1891): On the flow of water in open channels and pipes. In Transactions of the Institution of Civil Engineers of Ireland 20, pp. 161-207. 
Maris, T.; Cox, T.; Temmerman, S.; Vleeschauwer, P. de; van Damme, S.; Mulder, T. de et al. (2007): Tuning the tide. Creating ecological conditions for tidal marsh development in a flood control area. In Hydrobiologia 588 (1), pp. 31-43. DOI: 10.1007/s10750-007-0650-5.

Marsooli, Reza; Wu, Weiming (2014): Numerical investigation of wave attenuation by vegetation using a 3D RANS model. In Advances in Water Resources 74, pp. 245-257. DOI:

10.1016/j.advwatres.2014.09.012.

McFadden, Loraine; Spencer, Tom; Nicholls, Robert J. (2007): Broad-scale modelling of coastal wetlands. What is required? In Hydrobiologia 577 (1), pp. 5-15. DOI: 10.1007/s10750-006-0413-8.

Minden, Vanessa; Andratschke, Sandra; Spalke, Janina; Timmermann, Hanna; Kleyer, Michael (2012): Plant trait-environment relationships in salt marshes. Deviations from predictions by ecological concepts. In Perspectives in Plant Ecology, Evolution and Systematics 14 (3), pp. 183-192. DOI: 10.1016/j.ppees.2012.01.002.

Möller, I.; Spencer, T. (2002): Wave dissipation over macro-tidal saltmarshes. Effects of marsh edge typology and vegetation change. In Journal of Coastal Research 36, pp. 506-521. DOI: 10.2112/15515036-36.sp1.506.

Möller, Iris; Kudella, Matthias; Rupprecht, Franziska; Spencer, Tom; Paul, Maike; van Wesenbeeck, Bregje K. et al. (2014): Wave attenuation over coastal salt marshes under storm surge conditions. In Nature Geosci 7 (10), pp. 727-731. DOI: 10.1038/ngeo2251.

Morris, J. T.; Sundareshwar, P. V.; Nietch, C. T.; Kjerfve, B.; Cahoon, D. R. (2002): Responses of coastal wetlands to rising sea level. In Ecology 83 (10), pp. 2869-2877.

Mudd, Simon M.; D'Alpaos, Andrea; Morris, James T. (2010): How does vegetation affect sedimentation on tidal marshes? Investigating particle capture and hydrodynamic controls on biologically mediated sedimentation. In J. Geophys. Res. 115 (F3), p. 41. DOI: 10.1029/2009JF001566.

Müller, F.; Fischer, O. (1917): Das Wasserwesen an der schleswig-holsteinischen Nordseeküste. Teil 1, Die Halligen: 377.

Murray, Nicholas J.; Clemens, Robert S.; Phinn, Stuart R.; Possingham, Hugh P.; Fuller, Richard A. (2014): Tracking the rapid loss of tidal wetlands in the Yellow Sea. In Frontiers in Ecology and the Environment 12 (5), pp. 267-272. DOI: 10.1890/130260.

Nationalpark Wattenmeer (2004): Biotoptypen-Karten: Nationalpark Wattenmeer (Ed.), 2004.

Nerem, R. S.; Beckley, B. D.; Fasullo, J. T.; Hamlington, B. D.; Masters, D.; Mitchum, G. T. (2018): Climatechange-driven accelerated sea-level rise detected in the altimeter era. In Proceedings of the National Academy of Sciences of the United States of America 115 (9), pp. 2022-2025. DOI: 10.1073/pnas.1717312115.

Nerem, R. S.; Chambers, D. P.; Choe, C.; Mitchum, G. T. (2010): Estimating Mean Sea Level Change from the TOPEX and Jason Altimeter Missions. In Marine Geodesy 33 (sup1), pp. 435-446. DOI: 10.1080/01490419.2010.491031.

Neumeier, U.; Ciavola, P. (2004): Flow resistance and associated sedimentary processes in a Spartina maritima salt-marsh. In Journal of Coastal Research 20 (2), pp. 435-447.

Niemeyer, H. D.; Kaiser, R. (2001): Hydrodynamische Wirksamkeit von Lahnungen, Hellern und Sommerdeichen. In Die Küste 64, pp. 15-60. 
Nolte, S.; Koppenaal, E. C.; Esselink, P.; Dijkema, K. S.; Schuerch, M.; Groot, A. V. de et al. (2013a): Measuring sedimentation in tidal marshes. A review on methods and their applicability in biogeomorphological studies. In J Coast Conserv 17 (3), pp. 301-325. DOI: 10.1007/s11852-013-0238-3.

Nolte, Stefanie; Müller, Frauke; Schuerch, Mark; Wanner, Antonia; Esselink, Peter; Bakker, Jan P.; Jensen, Kai (2013b): Does livestock grazing affect sediment deposition and accretion rates in salt marshes? In Estuarine, Coastal and Shelf Science 135, pp. 296-305. DOI: 10.1016/j.ecss.2013.10.026.

Nyman, John A.; Walters, Russel J.; Delaune, Ronald D.; Patrick, William H. (2006): Marsh vertical accretion via vegetative growth. In Estuarine, Coastal and Shelf Science 69 (3-4), pp. 370-380. DOI: 10.1016/j.ecss.2006.05.041.

Oppenheimer, M.; B.C. Glavovic; J. Hinkel; R. van de Wal, A.K. Magnan, A. Abd-Elgawad, R. Cai, M. Cifuentes-Jara, R.M. DeConto, T. Ghosh, J. Hay, F. Isla, B. Marzeion, B. Meyssignac, and Z. Sebesvari (2019): Sea Level Rise and Implications for Low-Lying Islands, Coasts and Communities. In: IPCC Special Report on the Ocean and Cryosphere in a Changing Climate. In press. With assistance of H.-O. Pörtner, D.C. Roberts, V. Masson-Delmotte, P. Zhai, M. Tignor, E. Poloczanska, K. Mintenbeck, A. Alegría, M. Nicolai, A. Okem, J. Petzold, B. Rama, N.M. Weyer (eds.).

Pasternack, G. B.; Brush, G. S. (2002): Biogeomorphic controls on sedimentation and substrate on a vegetated tidal freshwater delta in upper Chesapeake Bay. In Geomorphology 43 (3-4), pp. 293-311.

Paul, M.; Bouma, T. J.; Amos, C. L. (2012): Wave attenuation by submerged vegetation. Combining the effect of organism traits and tidal current. In Mar. Ecol. Prog. Ser. 444, pp. 31-41. DOI: 10.3354/meps09489.

Peck, Erin K.; Wheatcroft, Robert A.; Brophy, Laura S. (2020): Controls on Sediment Accretion and Blue Carbon Burial in Tidal Saline Wetlands. Insights From the Oregon Coast, USA. In J. Geophys. Res. Biogeosci. 125 (2), p. 875. DOI: 10.1029/2019JG005464.

Peralta, G.; van Duren; Morris, E. P.; Bouma, T. J. (2008): Consequences of shoot density and stiffness for ecosystem engineering by benthic macrophytes in flow dominated areas. A hydrodynamic flume study. In Mar. Ecol. Prog. Ser. 368, pp. 103-115. DOI: 10.3354/meps07574.

Pérez-Harguindeguy, N.; Díaz, S.; Garnier, E.; Lavorel, S.; Poorter, H.; Jaureguiberry, P. et al. (2013): New handbook for standardised measurement of plant functional traits worldwide. In Australian Journal of Botany 61 (3).

Peterson, R. A. (2017): Estimating normalization transformations with bestNormalize.

Poerbandono, Poeberbandono; Mayerle, Roberto (2005): Composition and Dynamics of Sediments in Tidal Channels of the German North Sea Coast. In Die Küste 69, pp. 63-91.

Quedens, Georg (1991): Die Halligen. $10^{\text {th }}$ ed. Breklum Germany: Breklumer Verlag.

Reef, Ruth; Schuerch, Mark; Christie, Elizabeth K.; Möller, Iris; Spencer, Tom (2018): The effect of vegetation height and biomass on the sediment budget of a European saltmarsh. In Estuarine, Coastal and Shelf Science 202, pp. 125-133. DOI: 10.1016/j.ecss.2017.12.016.

Reise, K.; Baptist, M.; Burbridge, P.; Dankers, N.M.J.A.; Fischer, L.; Flemming, B. W. et al. (2010): The Wadden Sea - A Universally Outstanding Tidal Wetland. The Wadden Sea Quality Status Report Synthesis Report 2010. Common Wadden Sea Secretariat. Wilhelmshaven, Germany.

Reise, Karsten (2005): Coast of change. Habitat loss and transformations in the Wadden Sea. In Helgol Mar Res 59 (1), pp. 9-21. DOI: 10.1007/s10152-004-0202-6. 
Reuter, Rainer; Badewien, Thomas H.; Bartholomä, Alexander; Braun, Axel; Lübben, Andrea; Rullkötter, Jürgen (2009): A hydrographic time series station in the Wadden Sea (southern North Sea). In Ocean Dynamics 59 (2), pp. 195-211. DOI: 10.1007/s10236-009-0196-3.

Ricklefs, Klaus (1998): Die Verteilung von Schwebstoffen im Hörnum-Tief. In Die Küste 60, pp. 141-160.

Ridderinkhof, Herman; van der Ham, Ronald; van der Lee, Willem (2000): Temporal variations in concentration and transport of suspended sediments in a channel-flat system in the Ems-Dollard estuary. In Continental Shelf Research 20 (12-13), pp. 1479-1493. DOI: 10.1016/S0278-4343(00)00033-9.

Rupprecht, F.; Möller, I.; Paul, M.; Kudella, M.; Spencer, T.; van Wesenbeeck, B. K. et al. (2017): Vegetation-wave interactions in salt marshes under storm surge conditions. In Ecological Engineering 100, pp. 301-315. DOI: 10.1016/j.ecoleng.2016.12.030.

Scheffer, M.; Carpenter, S.; Foley, J.; Folke, C.; Walker, B. (2001): Catastrophic shifts in ecosystems. In Nature 413, pp. 591-596. DOI: 10.1038/35098000.

Schile, Lisa M.; Callaway, John C.; Morris, James T.; Stralberg, Diana; Parker, V. Thomas; Kelly, Maggi (2014): Modeling tidal marsh distribution with sea-level rise. Evaluating the role of vegetation, sediment, and upland habitat in marsh resiliency. In PloS one 9 (2), e88760. DOI:

10.1371/journal.pone.0088760.

Schindler, Malte (2014): Challenges and perspectives of the North Frisian Halligen Hooge, Langeness and Nordstrandischmoor - Marshland accretion and adaptation capacity to sea-level-rise. Dissertation. Georg-August-Universität Göttingen, Göttingen. Sedimentologie und Umweltgeologie, Geowissenschaftliches Zentrum der Universität Göttingen.

Schindler, Malte; Karius, Volker; Arns, Arne; Deicke, Matthias; Eynatten, Hilmar von (2014a): Measuring sediment deposition and accretion on anthropogenic marshland - Part II. The adaptation capacity of the North Frisian Halligen to sea level rise. In Estuarine, Coastal and Shelf Science 151, pp. 246-255. DOI: 10.1016/j.ecss.2014.08.027.

Schindler, Malte; Karius, Volker; Deicke, Matthias; Eynatten, Hilmar von (2014b): Measuring sediment deposition and accretion on anthropogenic marshland - Part I. Methodical evaluation and development. In Estuarine, Coastal and Shelf Science 151, pp. 236-245. DOI: 10.1016/j.ecss.2014.08.029.

Schindler, Malte; Willim, Katharina (2015): Berechnung langjähriger Überflutungshäufigkeiten für die Halligen Hooge, Langeneß und Nordstrandischmoor auf Basis regionaler Pegeldaten. In Coastline Reports 24, pp. 53-65.

Schuerch, M.; Vafeidis, A.; Slawig, T.; Temmerman, S. (2013): Modeling the influence of changing storm patterns on the ability of a salt marsh to keep pace with sea level rise. In J. Geophys. Res. Earth Surf. 118 (1), pp. 84-96. DOI: 10.1029/2012JF002471.

Schuerch, Mark; Rapaglia, J.; Liebetrau, V.; Vafeidis, A.; Reise, K. (2012): Salt Marsh Accretion and Storm Tide Variation. An Example from a Barrier Island in the North Sea. In Estuaries and Coasts 35 (2), pp. 486-500. DOI: 10.1007/s12237-011-9461-z.

Schuerch, Mark; Spencer, Tom; Temmerman, Stijn; Kirwan, Matthew L.; Wolff, Claudia; Lincke, Daniel et al. (2018): Future response of global coastal wetlands to sea-level rise. In Nature 561 (7722), pp. 231234. DOI: 10.1038/s41586-018-0476-5.

Seneviratne, Sonia I.; Nicholls, Neville; Easterling, David; Goodess, Clare M.; Kanae, Shinjiro; Kossin, James et al. (Eds.) (2012): Changes in Climate Extremes and their Impacts on the Natural Physical Environment. Managing the Risks of Extreme Events and Disasters to Advance Climate Change 
Adaptation: Special Report of the Intergovernmental Panel on Climate Change. With assistance of Field, C.B., V. Barros, T.F. Stocker, D. Qin, D.J. Dokken, K.L. Ebi, M.D. Mastrandrea, K.J. Mach, G.-K. Plattner, S.K. Allen,M. Tignor, and P.M. Midgley (eds.). Cambridge, UK, and New York, NY, USA: Cambridge University Press.

Shepard, Christine C.; Crain, Caitlin M.; Beck, Michael W. (2011): The protective role of coastal marshes. A systematic review and meta-analysis. In PloS one 6 (11), e27374. DOI: 10.1371/journal.pone.0027374.

Silva, H.; Dias, J. M.; Caçador, I. (2009): Is the salt marsh vegetation a determining factor in the sedimentation processes? In Hydrobiologia 621 (1), pp. 33-47. DOI: 10.1007/s10750-008-9630-7.

Smith, Carter S.; Gittman, Rachel K.; Neylan, Isabelle P.; Scyphers, Steven B.; Morton, Joseph P.; Joel Fodrie, F. et al. (2017): Hurricane damage along natural and hardened estuarine shorelines. Using homeowner experiences to promote nature-based coastal protection. In Marine Policy 81 (10), pp. 350358. DOI: 10.1016/j.marpol.2017.04.013.

Soulsby, Richard (1997): Dynamics of marine sands. A manual for practical applications. London: Thomas Telford.

Stanev, E. V.; Badewien, T. H.; Freund, H.; Grayek, S.; Hahner, F.; Meyerjürgens, J. et al. (2019): Extreme westward surface drift in the North Sea. Public reports of stranded drifters and Lagrangian tracking. In Continental Shelf Research 177, pp. 24-32. DOI: 10.1016/j.csr.2019.03.003.

Sternberg, R. W.; Johnson, R. V.; Cacchione, D. A.; Drake, D. E. (1986): An instrument system for monitoring and sampling suspended sediment in the benthic boundary layer. In Marine Geology 71, 1986 (3-4), pp. 187-199. DOI: 10.1016/0025-3227(86)90069-1.

Stock, Martin (2011): Patterns in surface elevation change across a temperate salt marsh platform in relation to sea-level rise. In Coastline Reports 17 (3), pp. 33-48.

Stoddart, D. R.; Reed, D., J.; French, J. R. (1989): Understanding salt-marsh accretion, Scolt Head Island, Norfolk, England. In Estuaries 12 (4), pp. 228-236.

Strickler, A. (Ed.) (1923): Beiträge zur Frage der Geschwindigkeitsformel und der Rauhigkeitszahlen für Ströme, Kanäle und geschlossene Leitungen. Bern, Switzerland: Eidg. Amt für Wasserwirtschaft.

Suchrow, Sigrid; PohImann, Nina; Stock, Martin; Jensen, Kai (2012): Long-term surface elevation changes in German North Sea salt marshes. In Estuarine, Coastal and Shelf Science 98, pp. 71-83. DOI:

10.1016/j.ecss.2011.11.031.

Sutherland, T.F; Lane, P.M; Amos, C.L; Downing, J. (2000): The calibration of optical backscatter sensors for suspended sediment of varying darkness levels. In Marine Geology 162 (2-4), pp. 587-597. DOI: 10.1016/S0025-3227(99)00080-8.

Temmerman, S.; Bouma, T. J.; Govers, G.; Lauwaet, D. (2005a): Flow Paths of Water and Sediment in a Tidal Marsh: Relations with Marsh Developmental Stage and Tidal Inundation Height. In Estuaries 28 (3), pp. 338-352.

Temmerman, S.; Bouma, T. J.; Govers, G.; Wang, Z. B.; Vries, M. B. de; Herman, P. M. J. (2005b): Impact of vegetation on flow routing and sedimentation patterns. Three-dimensional modeling for a tidal marsh. In J. Geophys. Res. 110 (F4), n/a-n/a. DOI: 10.1029/2005JF000301.

Temmerman, S.; Bouma, T. J.; van de Koppel, J.; van der Wal, D.; Vries, M. B. de; Herman, P. M. J. (2007): Vegetation causes channel erosion in a tidal landscape. In Geology 35 (7), pp. 631-634. 
Temmerman, S.; Govers, G.; Meire, P.; Wartel, S. (2003a): Modelling long-term tidal marsh growth under changing tidal conditions and suspended sediment concentrations, Scheldt estuary, Belgium. In Marine Geology 193 (1-2), pp. 151-169. DOI: 10.1016/S0025-3227(02)00642-4.

Temmerman, S.; Govers, G.; Wartel, S.; Meire, P. (2003b): Spatial and temporal factors controlling shortterm sedimentation in a salt and freshwater tidal marsh, Scheldt estuary, Belgium, SW Netherlands. In Earth Surf. Process. Landforms 28 (7), pp. 739-755. DOI: 10.1002/esp.495.

Temmerman, S.; Govers, G.; Wartel, S.; Meire, P. (2004): Modelling estuarine variations in tidal marsh sedimentation. Response to changing sea level and suspended sediment concentrations. In Marine Geology 212 (1-4), pp. 1-19. DOI: 10.1016/j.margeo.2004.10.021.

Temmerman, Stijn; Meire, Patrick; Bouma, Tjeerd J.; Herman, Peter M. J.; Ysebaert, Tom; Vriend, Huib J. de (2013): Ecosystem-based coastal defence in the face of global change. In Nature 504 (7478), pp. 7983. DOI: $10.1038 /$ nature12859.

Temmerman, Stijn; Moonen, Pieter; Schoelynck, Jonas; Govers, Gerard; Bouma, Tjeerd J. (2012): Impact of vegetation die-off on spatial flow patterns over a tidal marsh. In Geophys. Res. Lett. 39 (3), n/a-n/a. DOI: 10.1029/2011GL050502.

Thonon, Ivo; Jong, Kor de; van der Perk, Marcel; Middelkoop, Hans (2007): Modelling floodplain sedimentation using particle tracking. In Hydrol. Process. 21 (11), pp. 1402-1412. DOI: 10.1002/hyp.6296.

van der Wal, Daphne; Pye, Kenneth (2004): Patterns, rates and possible causes of saltmarsh erosion in the Greater Thames area (UK). In Geomorphology 61 (3-4), pp. 373-391. DOI:

10.1016/j.geomorph.2004.02.005.

van Eerdt, M. M. (1985): Salt marsh cliff stability in the oosterschelde. In Earth Surf. Process. Landforms 10 (2), pp. 95-106.

van Wijnen, H. J.; Bakker, J. P. (2001): Long-term Surface Elevation Change in Salt Marshes. A Prediction of Marsh Response to Future Sea-Level Rise. In Estuarine, Coastal and Shelf Science 52 (3), pp. 381-390. DOI: $10.1006 /$ ecss.2000.0744.

Vandenbruwaene, W.; Maris, T.; Cox, T.J.S.; Cahoon, D. R.; Meire, P.; Temmerman, S. (2011): Sedimentation and response to sea-level rise of a restored marsh with reduced tidal exchange. Comparison with a natural tidal marsh. In Geomorphology 130 (3-4), pp. 115-126. DOI: 10.1016/j.geomorph.2011.03.004.

Verney, Romaric; Lafite, Robert; Brun-Cottan, Jean-Claude (2009): Flocculation Potential of Estuarine Particles. The Importance of Environmental Factors and of the Spatial and Seasonal Variability of Suspended Particulate Matter. In Estuaries and Coasts 32 (4), pp. 678-693. DOI: 10.1007/s12237-0099160-1.

Voulgaris, George; Meyers, Samuel T. (2004): Temporal variability of hydrodynamics, sediment concentration and sediment settling velocity in a tidal creek. In Continental Shelf Research 24 (15), pp. 1659-1683. DOI: 10.1016/j.csr.2004.05.006.

Vriend, Huib J. de; van Koningsveld, Mark; Aarninkhof, Stefan G.J.; Vries, Mindert B. de; Baptist, Martin J. (2015): Sustainable hydraulic engineering through building with nature. In Journal of Hydroenvironment Research 9 (2), pp. 159-171. DOI: 10.1016/j.jher.2014.06.004. 
Wahl, T.; Haigh, I. D.; Woodworth, P. L.; Albrecht, F.; Dillingh, D.; Jensen, J. et al. (2013): Observed mean sea level changes around the North Sea coastline from 1800 to present. In Earth-Science Reviews 124, pp. 51-67. DOI: 10.1016/j.earscirev.2013.05.003.

Wahl, Thomas; Jensen, Jürgen; Frank, Torsten; Haigh, Ivan David (2011): Improved estimates of mean sea level changes in the German Bight over the last 166 years. In Ocean Dynamics 61 (5), pp. 701-715. DOI: 10.1007/s10236-011-0383-x.

Warren, I. R.; Bach, H. K. (1992): MIKE 21: a modelling system for estuaries, coastal waters and seas. In Environmental Software 7, pp. 229-240. DOI: 10.1016/0266-9838(92)90006-P.

Watson, Elizabeth Burke; Wigand, Cathleen; Davey, Earl W.; Andrews, Holly M.; Bishop, Joseph; Raposa, Kenneth B. (2017): Wetland loss patterns and inundation-productivity relationships prognosticate widespread salt for southern New England. In Estuaries and coasts : journal of the Estuarine Research Federation 40 (3), pp. 662-681. DOI: 10.1007/s12237-016-0069-1.

Whitehouse, R.; Soulsby, R.; Roberts, W.; Mitchener, H. (2000): Dynamics of estuarine muds. A manual for practical applications. London: Thomas Telford.

Widdows, J.; Pope, N. D.; Brinsley, M. D. (2008): Effect of Spartina anglica stems on near-bed hydrodynamics, sediment erodability and morphological changes on an intertidal mudflat. In Mar. Ecol. Prog. Ser. 362, pp. 45-57. DOI: 10.3354/meps07448.

Wolff, W. J.; Bakker, J. P.; Laursen, K.; Reise, K. (2010): The Wadden Sea Quality Status Report - Synthesis Report 2010. Wadden Sea Ecosystem No. 29. In Wadden Sea Ecosystem 29, pp. 25-74.

WOR1 (2010): World Ocean Review 1. Mit den Meeren leben - ein Bericht über den Zustand der Weltmeere. Die ungewisse Zukunft der Küsten. Edited by maribus gGmbH. Hamburg. 


\section{Research design}

\section{Planning and implementation}

When I joined the research project Living Coast Lab on 17 October 2016, the main purpose of the study to assess the turbidity around Langeness with optical measurements was already arranged by Hilmar von Eynatten and Volker Karius, who generally planned the geoscientific part of the research project Living Coast Lab. Together with Jörg Gutkuhn from ARGUS Environmental Instruments and Volker Karius we devised a prototype of a turbidity measurement system that would serves our purposes. Using this prototype, I built seven additional measurement stations containing data acquisition and transmission units as well as cabling to implement the network of turbidity measurement stations. The installation of the whole turbidity measurement network in the field was done with Jörg Gutkuhn and Volker Karius. Artiom Patrinica from AVP-Systeme helped us to network our whole turbidity measurement station network.

\section{Field work}

After the field instruction by Volker Karius, I was responsible for the field work including installation of the whole turbidity measurement network and the sampling of sediment traps after each inundation. In addition, sediment accumulators had to be emptied before an inundation and the turbidity measurement stations had to be checked. Together with Julia Bass from the institute of evolutionary ecology and conservation genomics of the University Ulm, I installed additional 81 sediment traps during the first winter season 2016/17.

\section{Model set-up}

The set-up of the detailed hydrodynamic numerical model was carried out by Sebastian Niehüser from the research Institute for Water and Environment of the University of Siegen with the software package Mike $21^{\circledR}$ by DHI-WASY. The large scale hydrodynamic model of the North Sea to create the boundary conditions for our detailed hydrodynamic model was provided by Arne Arns from the faculty of agricultural and environmental sciences of the University of Rostock. 


\section{Data generation}

I was responsible for the data generation generated by the turbidity measurement network including turbidity measurement stations, sediment traps and sediment accumulators and the maintenance of the turbidity measurement network during the whole measuring campaign. After the laboratory instruction by Volker Karius, I carried out the sediment sample preparation and the related measurements and analysis of grain sizes as well as the quantifying of total mass of solids, clastic and organic components. Julia Bass assembled a large data set of accretion rates, plant abundance, and trait measurements from the German Wadden Sea. For the modeling, I adjusted the detailed model grid of the coastal protection measures with Mike21 ${ }^{\circledR}$ and Matlab 2017a to generate different hydrodynamic scenarios. In the following I performed the particle tracking with Mike $21^{\circledR}$.

\section{Data Analysis}

In order to analyze the turbidity data, I developed a calibration procedure with a self-built calibration tank and organized a boat trip from which in situ water samples were collected in the vicinity of the turbidity stations on tidal flats to verify the accuracy of the calibrated data. The post processing and analysis of the turbidity data as well as the processing and analysis of wind, wave and gauge level data was done by me exclusively with Matlab 2017a. For the modelling, I analysed the hydrodynamics as well as particle tracking data with Matlab 2017a.

\section{Data interpretation}

The data interpretation was carried out with close exchange with both supervisors Hilmar von Eynatten and Volker Karius. For the modelling, an additional close exchange with Sebastian Niehüser was maintained. The interpretation of Julia Bass's data set was done without my assistance.

\section{Manuscript-writing}

As main author I wrote the manuscripts (Hache et al. 2019, 2020, 2021, submitted). The modelling manuscript, Sebastian Niehüser contributed partly to the method part. Julia Bass wrote the manuscript Bass et al. (in prep.).

\section{Manuscript-review and editing}

In general, all manuscripts (Hache et al. 2019, 2020, 2021, submitted) were carefully reviewed and edited by both supervisors Hilmar von Eynatten and Volker Karius. The modelling 
manuscript (Hache et al. 2021) was also checked and partially improved prior to submission by Sebastian Niehüser and Arne Arns. The manuscript from Julia Bass (Bass in prep.) was carefully reviewed by Volker Karius and partially by me.

\section{Final bachelor theses during the project}

\section{As assistance}

Judith Sophie Carstens and Anna-Lena Rüßmann (2018) „Wattkartierung um Hallig Langeness Bestimmung der Korngrößenverteilung, Karbonatgehalt und organischem Kohlenstoff an ausgewählten Profilen"

Dorothee Fehling (2018) „Analyse der Schwebstoffkonzentration um Hallig Langeness in Abhängigkeit von hydrologischen und meteorologischen Parametern sowie der Korngröße“

\section{As assessor}

Ernst-Moritz Gröschl (2020) „Umlagerung und Erosion von „Land unter" Sedimenten auf Hallig Langeness - Anwendung von Muschelschill und Fluorit als anorganische Tracer" 


\section{Danksagung}

Zuallererst möchte ich mich bei Prof. Dr. Hilmar von Eynatten und Dr. Volker Karius dafür bedanken, mich für das Projekt ausgewählt zu haben. Danke auch für euer Vertrauen, mit dem ihr mich zu akademischen Würden begleitet habt. Für den regen Austausch über die Halligwelt möchte ich mich bei Dr. Matthias Deicke bedankten. Der ganzen Abteilung möchte ich meinen Dank für die so warme und herzliche Atmosphäre über die Zeit meiner Promotion aussprechen.

Großer Dank gebührt ebenfalls allen Mitarbeitern der Metallwerkstatt für ihr unglaubliches Engagement, mit dem sie meine Belange mit eigenen Ideen aufgewertet und tadellos umgesetzt haben. Ich möchte mich bei Jörg Gutkuhn von ARGUS Environmental Instruments für seine geduldige und liebenswerte Unterstützung beim Umgang mit Messsystemen und ihren Eigenarten bedanken.

Bei den Bewohnern von Langeneß möchte ich mich für ihre Gastfreundschaftlichkeit, Geduld und Unterstützung bei der Verwirklichung des Projektes bedanken. Einen besonderen Dank möchte ich hier der Familie Johannsen aussprechen, die mich bei sämtlichen Belangen und brenzligen Situationen tatkräftig unterstützt hat. Weiter möchte ich mich bei Thorsten Seitel für die Reparatur meines Werkzeuges und die Bereitstellung und Führung seines Bootes bedanken. Danke auch an Hans-Werner Johannsen für so manchen wärmenden Schluck an kalten wie auch an warmen Tagen.

Lieben Dank an meine ganze Familie. Ganz besonders möchte ich mich bei meiner Frau Caren für ihre aufopfernde Hilfe, Geduld und Liebe bedanken. Meinen Söhnen Finn und Theo möchte ich abschließend sagen: Papa hat euch lieb! 